\title{
PROCESS PLANNING FOR MULTI-AXIS ADDITIVE MANUFACTURING
}


Cover design: Dörte Bockwoldt

Printed by: IPSKAMP Printing Enschede

Layout: David de Groot, persoonlijkproefschrift.nl

ISBN: 978-90-365-5209-7

DOI: $10.3990 / 1.9789036552097$

(c) 2021 September, The Netherlands. All rights reserved. No parts of this thesis may be reproduced, stored in a retrieval system or transmitted in any form or by any means without permission of the author. Alle rechten voorbehouden. Niets uit deze uitgave mag worden vermenigvuldigd, in enige vorm of op enige wijze, zonder voorafgaande schriftelijke toestemming van de auteur. 
PROCESS PLANNING FOR MULTI-AXIS ADDITIVE MANUFACTURING

\section{DISSERTATION}

to obtain

the degree of doctor at the University of Twente, on the authority of the rector magnificus,

prof. dr. ir. Tom Veldkamp,

on account of the decision of the Doctorate Board,

to be publicly defended

on $16^{\text {th }}$ of September 2021, at 14:45

by

Dipl.-Ing. Yavuz Murtezaoglu

born on the 27th of June 1970

in Istanbul, Turkey 
This dissertation has been approved by:

\section{Supervisor(s):}

prof. dr. Ian Gibson

Co-supervisor(s):

dr. ir. T.H.J. Vaneker

\section{Graduation committee:}

Chairman/secretary

Prof. dr. ir. Bart Koopman

Supervisor

prof. dr. Ian Gibson

Co-supervisor

dr. ir. T.H.J. Vaneker

Members

prof. dr.ir. Bert Lauwers

KU Leuven, Mechanical Engineering

assoc. prof. Pascal Etman

TU Eindhoven, Mechanical Engineering

prof. dr. ing. Bojana Rosic

Universiteit Twente, ET, Applied Mechanics

prof. dr. ir. Gert-Willem Römer

Universiteit Twente, ET, Laser Processing

prof. dr. ing. Sebastian Thiede

Universiteit Twente, ET, Design Engineering 


\section{ACKNOWLEDGEMENT}

Throughout the writing of this dissertation I have received a great deal of support and assistance.

I would first like to thank my supervisor, Ian Gibson and my co-supervisor, Tom Vaneker, whose expertise was invaluable in formulating the research questions and methodology. Your insightful feedback pushed me to sharpen my thinking and brought my work to a higher level.

I would like to acknowledge my colleagues at ModuleWorks from Aachen, for their wonderful collaboration. I would particularly like to single out Denys Plakhotnik. I want to thank you for your support and great discussions.

In deep sadness, I would like to name here Fred van Houten, who passed away on October $18^{\text {th }}, 2020$. My journey to write this thesis started with him in 2017.

In addition, I would like to thank my family for their great support during this intense time. You are always there for me. 


\section{ABSTRACT}

In recent decades additive manufacturing has transformed from a set of prototyping methods to a mature set of industrial level manufacturing processes. To achieve predictable and high-quality end-use products, these processes require the application of high-quality process planning solutions.

This research questions the common perception that the nature of additive manufacturing processes has led to making process planning for additive manufacturing a trivial step. It is shown that duplicating process planning strategies from subtractive process planning algorithms leads to suboptimal results for their additive counterparts. The main focus of the research is thus the development of multi-axis process planning strategies that take the specific requirements of direct material deposition techniques (FDM, DED) into account.

The research presented in this thesis starts with the state of art on multi-axis additive manufacturing. CNC based subtractive manufacturing strategies and established subtractive process planning steps and terminology are used as a starting point to address the specific challenge of process planning for additive manufacturing. The next chapters describe the development of two novel algorithms for multi-axis additive manufacturing path planning. The first algorithm is based on geometry decomposition that partitions the main geometry into sections with similar functional requirements. Within a partition, material is deposited using constant printing settings, while between the partitions the main deposition direction is optimized. The second algorithm is focused on calculating continuous multi-axis tool paths following the part surface geometry, thus preserving smooth motion of nozzle orientation and collision freeness of the additive manufacturing process. Simulations and physical experiments have been executed to validate the strategies proposed. The developed algorithm for multi-axis additive manufacturing reduced the surface roughness due to stair-case effect by $57 \%$ compared to $2.5 \mathrm{D}$ AM for the test part used. 


\section{SAMENVATTING}

Gedurende de laatste decennia heeft additive manufacturing zich ontwikkeld van een methode voor het creëren van prototypes tot een volwassen en industrieel toepasbare set van productietechnieken. Om te komen tot voorspelbare en hoogwaardige producten is gebruik van hoogwaardige proces planning algoritmes noodzakelijk.

Dit onderzoek betwist het breed aangehangen idee dat de aard van additive manufacturing processen de proces planning stap heeft gereduceerd tot een triviale stap. Er wordt aangetoond dat het kopiëren van proces-planningsalgoritmen van subtractieve processen leidt tot suboptimale resultaten voor hun additieve tegenhangers. De focus van dit onderzoek richt zich op de ontwikkeling van meerassige proces planning strategieën die de specifieke eisen van directe depositie additive manufacturing technieken (FDM, DED) in acht neemt.

Het in deze thesis gepresenteerde onderzoek begint met de beschrijving van de state-of-the-art betreffende meerassige additive manufacturing. CNC gebaseerde subtractieve productie strategieën en erkende subtractieve proces planning stappen en terminologieën worden gebruikt als startpunt voor de ontwikkeling van specifieke strategieën voor additive manufacturing. De volgende hoofdstukken beschrijven de ontwikkeling van twee nieuwe algoritmen voor meerassige additive manufacturing gereedschapsbaan planning. Het eerste algoritme is gebaseerd op decompositie van de geometrie in secties met ongeveer vergelijkbare functionele eisen. Binnen een partitie wordt materiaal gedeponeerd terwijl er gebruik wordt gemaakt van niet variërende print instellingen. Tussen de partities kan de depositie richting worden aangepast. Het tweede algoritme richt zich op de berekening van continue meerassige, het oppervlak van het onderdeel volgende, gereedschapsbanen. Hierbij wordt de vloeiende orientatie van het depositiegereedschap en het efficiënt voorkomen van botsingen tussen het gereedschap en het product als optimalisatiecriteria meegenomen. Simulaties en experimenten zijn uitgevoerd om de voorgestelde strategieën te valideren. Het ontwikkelde algoritme voor meerassige additive manufacturing verminderde de oppervlakteruwheid als gevolg van het trap-case-effect met $57 \%$ in vergelijking met 2.5D AM voor het gebruikte testdeel. 


\section{CONTENTS}

1

$\begin{array}{lll}1.1 & \text { How and why additive manufacturing developed } & 16\end{array}$

$\begin{array}{llr}1.2 & 19\end{array}$

$\begin{array}{llr}\text { 1.2.1 Step 1: CAD } & 20\end{array}$

$\begin{array}{lll}\text { 1.2.2 Step 2: Conversion to STL } & 20\end{array}$

$\begin{array}{ll}\text { 1.2.3 Step 3: Transfer to AM machine and STL file manipulation } & 21\end{array}$

$\begin{array}{lll}\text { 1.2.4 Step 4: Machine setup } & 21\end{array}$

$\begin{array}{lll}1.2 .5 & \text { Step 5: Build } & 21\end{array}$

$\begin{array}{llr}\text { 1.2.6 Step 6: Removal } & 21\end{array}$

$\begin{array}{lll}\text { 1.2.7 Step 7: Post-processing } & 21\end{array}$

$\begin{array}{lll}\text { 1.2.8 Step 8: Application } & 21\end{array}$

1.3 Overview of main AM process groups 22

$\begin{array}{lll}\text { 1.3.1 Material extrusion } & 23\end{array}$

$\begin{array}{lll}\text { 1.3.2 } & \text { Powder bed fusion } & 25\end{array}$

$\begin{array}{ll}\text { 1.3.3 Vat photopolymerization } & 27\end{array}$

$\begin{array}{lr}1.3 .4 \quad \text { Material jetting } & 29\end{array}$

$\begin{array}{llr}1.3 .5 & \text { Binder jetting } & 31\end{array}$

$\begin{array}{lll}\text { 1.3.6 Sheet lamination } & 33\end{array}$

$\begin{array}{lll}\text { 1.3.7 Directed-energy deposition } & 35\end{array}$

1.3.8 Types of the AM processes 36

1.4 Social and economic relevance of additive

1.5 Challenges in process planning for additive manufacturing 4

1.5.1 Challenge: elimination or optimization of the use of support structures 41

1.5.2 Challenge: improvement of the part surface quality 42

1.5.3 Challenge and solution: development of an automated multi-axis AM process planning 43

$\begin{array}{lll}1.6 & \text { Research questions and methodology }\end{array}$

2 STATE OF THE ART ON PART DECOMPOSITION AND PATH PLANNING FOR MULTI-AXIS DECOMPOSITION $\quad 49$

2.1 Path planning for multi-axis AM, known experiments from the literature $\quad 50$

$\begin{array}{lll}2.1 .1 & \text { Basic decomposition strategies } & 50\end{array}$

2.1.2 Overview and classification of multi-axis path planning for AM 56

$\begin{array}{llr}2.2 & 67 \\ & \text { Conclusion } & 67\end{array}$ 
3.1 Development of automatic $3+2$ axis AM process planning for non-branching shapes 75

3.1.1 Algorithmic problem definition 76

3.1.2 Algorithmic premises for geometric analysis of meshes 78

3.2 Full-volume decomposition based on half-spaces 86

3.2.1 Point cloud approximation of meshes 89

3.2.2 Multi-plane decomposition 91

3.2.3 Search for a split-plane position $\quad 92$

$\begin{array}{lll}\text { 3.2.4 Algorithm summary } & 94\end{array}$

3.2.5 Decomposition examples 98

3.3 Greedy volume split/decomposition based on half-spaces 106

$\begin{array}{ll}\text { 3.3.1 Construction of the search space } & 108\end{array}$

3.3.2 Search of the splitting plane 109

3.3.3 Applying different conditions 111

$\begin{array}{lll}3.4 & \text { Printing experiments using the developed algorithm } & 115\end{array}$

$\begin{array}{lll}3.5 & \text { Conclusions } & 123\end{array}$

\begin{tabular}{lll}
4 & TRUE MULTI-AXIS AM TOOLPATHS & 127 \\
\hline
\end{tabular}

$\begin{array}{lll}4.1 & \text { Generation of curved slices to fill a given volume } & 129\end{array}$

$\begin{array}{ll}\text { 4.1.1 Offsetting and trimming of NURBS } & 130\end{array}$

$\begin{array}{lll}\text { 4.1.2 Offsetting of meshes } & 131\end{array}$

4.2 Planning of the nozzle orientations for DED $\quad 147$

4.2.1 Redistribution of toolpath points and initialization of a collision-free toolpath $\quad 147$

4.2.2 Toolpath smoothing 150

4.2.3 Experimental validation (single track with smoothing and collision avoidance) 153

4.2.4 Experimental validation (geodesic and offset layers) 164

$\begin{array}{lll}4.3 & \text { Conclusion } & 169\end{array}$

$5 \quad$ CONCLUSIONS AND OUTLOOK 173

$\begin{array}{lll}5.1 & \text { Conclusions } & 174\end{array}$

$\begin{array}{lll}5.2 & \text { Outlook } & 174\end{array}$ 


\section{TABLE OF ABBREVIATIONS}

\begin{tabular}{|c|c|}
\hline Abbreviation & Full form \\
\hline $3 D$ & three-dimensional \\
\hline $2 \mathrm{D}$ & two-dimensional \\
\hline$A A B B$ & axis-aligned bounding box \\
\hline ABS & acrylonitrile butadiene styrene \\
\hline AM & additive manufacturing \\
\hline AMF & additive manufacturing file format \\
\hline BSP & binary space partitioning \\
\hline BJ & binder jetting \\
\hline CAD & computer aided design \\
\hline CAM & computer aided manufacturing \\
\hline CFD & computational fluid dynamics \\
\hline CLIP & continuous liquid interface production \\
\hline $\mathrm{CNC}$ & computer numeric control \\
\hline $\mathrm{CT}$ & computer tomography \\
\hline DED & direct energy deposition \\
\hline DLP & digital light processing \\
\hline DMD & digital mirror device \\
\hline DMLS & direct metal laser sintering \\
\hline FDM & fused deposition modelling \\
\hline GUI & graphic user interface \\
\hline LOM & laminated object manufacturing \\
\hline LPBF & laser powder bed fusion \\
\hline NURBS & non-uniform rational basis spline \\
\hline PA & polyamide \\
\hline PBF & powder bed fusion \\
\hline PC & polycarbonate \\
\hline PEEK & polyetheretherketone \\
\hline PLA & polylactic acid \\
\hline $\mathrm{SCL}$ & spherically curved layer \\
\hline SGC & solid ground curing \\
\hline SHS & selective heat sintering \\
\hline SLERP & spherical linear interpolation \\
\hline SLA & stereolithography \\
\hline SLM & selective laser melting \\
\hline SLS & selective laser sintering \\
\hline STL & standard triangle language \\
\hline UV & ultraviolet \\
\hline
\end{tabular}




\section{LIST OF SYMBOLS}

Symbol

(1), (2) and (3)

$\alpha_{i}$

\begin{tabular}{c}
$\alpha_{\text {threshold }}$ \\
$\delta \delta$ \\
\hline$\varphi$ \\
$\theta$ \\
\hline$A_{i}$ \\
\hline$A_{i}^{\prime}$ \\
\hline
\end{tabular}

$\boldsymbol{C}_{j}$ and $\boldsymbol{C}_{1} \ldots \boldsymbol{C}_{\boldsymbol{8}}$

$C_{w_{1}} \ldots C_{W_{7}}$

$d$

$\begin{array}{ll}d_{c} & \mathrm{~m}\end{array}$

$d_{c}^{\prime} \quad \mathrm{m}$

$d_{\text {global,max }}$

$d_{L}$

$d_{L F O}$ and $d_{L F 1}$

dmin and dmax

$d_{\text {offset }}$

$E, E_{1}, E^{\prime}, E_{i}^{\prime \prime}$, and $E_{i-1}$

$E_{0}$

$E_{(1,2)}$

$E_{(2), 3}$

$F_{\mathrm{mi}}, F_{1}, F_{2}$, and $F_{1, i}$

$F_{(1,2)}$ and $F_{(2),(3)}$

i

K

l

$L_{W_{1}} \ldots L_{W_{4}}$

$n$

$n_{\text {desired repositioning }}$

$\overrightarrow{\mathbf{n}}_{i}$ and $\overrightarrow{\mathbf{n}}_{1, i}$

$n_{\text {layers }}$

$P_{E}$

$\boldsymbol{P}_{i}, \boldsymbol{P}_{i, j}$

$r$

$\overrightarrow{\mathbf{r}}_{1}, \overrightarrow{\mathbf{r}}_{2}$

$S$

$t$

$T_{1, i}$

$T_{2, i}$

$u$
Units Description

half-plane indices

rad angle between the triangle normal and printing direction

rad threshold angle

m voxel width

rad Euler angle

rad Euler angle

$\mathrm{m}^{2} \quad$ area of $i$ th triangle

$\mathrm{m}^{2}$ area of a fraction of $i$ th triangle

corners (3D points) of an AABB

$\mathrm{m}$ values of circular windows in distance field computation

distance

distance from a vertex point in circular windows

distance from a point within a triangle in circular windows

globally defined distance

distance from the baseline to a point on linear windows

$\mathrm{m}$ widths of laser spot

$\mathrm{m}$ distance range boundaries

$m$ offset distance

split planes

split plane at the default position

split plane between 1 st and 2 nd half-spaces

split plane between 2 nd and 3rd half-spaces

error functions

error functions between the by-planes between specified half-spaces

index for counting

penalty coefficient

m layer thickness

$m$ values of linear windows in distance field computation

number of items

desired number of $3+2$ operations

normal vectors of $i$ th triangle

number of layers

3D point on the split plane $E$

3D point

$m$ search radium around a voxel

plane orientation vectors

3D point defining the split plane $E$

interpolation parameter

ith triangle object in half-space (1)

ith triangle object in half-space (2)

parametric coordinate 
Symbol

\begin{tabular}{|c|c|c|}
\hline Symbol & Units & Description \\
\hline$v$ & & parametric coordinate \\
\hline V & & voxel \\
\hline $\boldsymbol{v} \mathbf{0}$ and $\boldsymbol{v} \mathbf{1}$ & & nozzle orientation vectors at key points \\
\hline$w$ & & parametric coordinate \\
\hline$x$ & & Cartesian coordinate \\
\hline$y$ & & Cartesian coordinate \\
\hline$Z$ & & Cartesian coordinate \\
\hline$z_{\max }$ and $z_{\min }$ & $\mathrm{m}$ & vertical extents of the working space \\
\hline
\end{tabular}






\section{CHAPTER 1}

| Introduction 
Additive manufacturing (AM) refers to fabrication methods that use digital data to deposit material in a desired shape. Like other technologies, AM has its own strengths and weaknesses. However, it is still under rapid development and is far from reaching its mature state. So, there are many challenges to tackle and plenty of opportunities to optimize the process.

This chapter provides a brief introduction to manufacturing and explains how AM has evolved in the recent decades into an individual branch of manufacturing technologies. Further in this chapter, the social and economic relevance of additive manufacturing is discussed. Although the research and larger scale deployment of this manufacturing method has been very dynamic in the last decade, future expectations are still much higher. Following that, some of the challenges in this field are presented. At the end of this chapter, an overview of the research questions of this work is presented and the chosen research methodology is explained.

\subsection{HOW AND WHY ADDITIVE MANUFACTURING DEVELOPED}

A long time ago, our prehistoric ancestors entered the Stone Age. One of the most distinct signs of the Stone Age was the use of stone tools. Although it is not possible to determine exactly when these hominids started to use shaped stones as tools, many archeological findings suggest that the tools had already been in use for millions of years. The earliest known artifacts were discovered in 2015 in Kenya . They were named "Lomekwi" after the name of the archeological excavation site in which they were found and date back 3.3 million years. Some examples are shown in Figure 1. These tools were made by hammering one stone with another to create sharp flakes. Usually, the more advanced stone tools were bifacial, almond-shaped lithic flakes that are believed to be elements of prehistoric tools, like hand axes or knives.

Figure 1: Photographs of selected artifacts from the Lomekwi archeological site site (Harmand, et al., 2015)
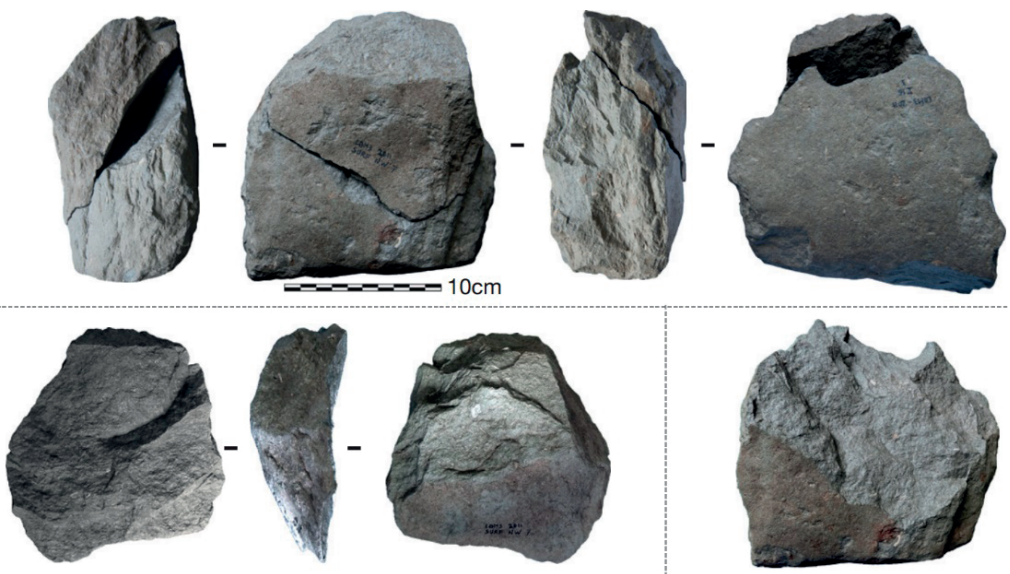
When the Stone Age ended, the world transitioned to the Copper, Bronze and Iron Ages, respectively. Mankind learned how to smelt and process copper, iron and their alloys. Perhaps, as a byproduct of making fire, the bow drill, also called a "fire drill," was invented as a "machine tool." It was used for drilling but also as a type of vertical lathe, as shown in Figure 2a. In the tomb of King Tutankhamun (1350 B.C.), numerous machined round tenon joints were found, and these are believed to be the earliest known parts made with a lathe. To operate a lathe, one person has to pull a cord back and forth to spin a wooden stick while another cuts away excess wood by holding a cutter and moving it along the rotating wooden stick. The Stone Age ended not because the humans ran out of stones but because new materials and new technologies like this had been discovered.

Figure 2: Depictions of early manufacturing practices

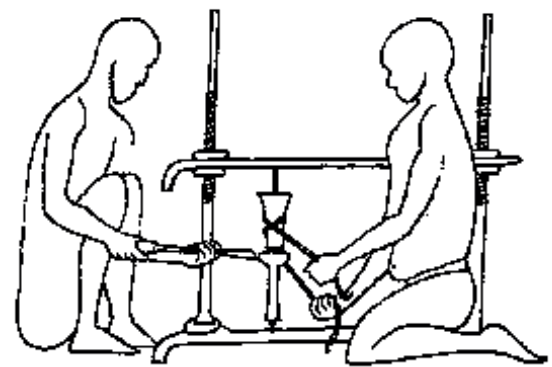

a) Sketch of Egyptian lathe (circa 300 B.C.) (Watson, 1994)

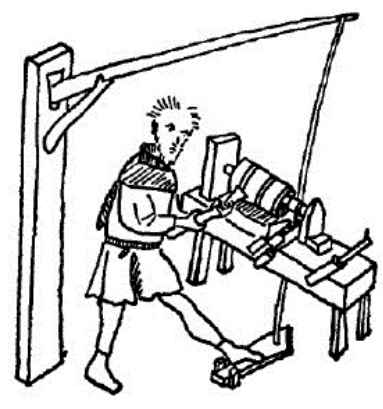

b) Pole lathe (Daumas, 1970)

A further significant improvement of the lathe was to eliminate one of the lathe operators and free the operator's hands. This lathe modification, called the pole lathe, is depicted in Figure $2 b$. Although this was a major technological breakthrough, it is not much more complicated than the bow lathe. The driving cord is connected to a flexible pole and a simple treadle beneath the bed of the lathe. The lathe operator must pedal the treadle to pull the cord and spin the workpiece. Then, the flexible pole springs back and returns the cord to the initial position. The pole lathe not only eliminated the need for an assistant but also improved the quality of the produced parts because the pole lathe was more rigid and substantial in its construction than earlier versions.

It is not known when or where the pole lathe first appeared, but it is estimated that it was invented sometime before the 13th century A.D., since there are at least two clear sources from that time (a stained glass window in Chartres Cathedral and an image in Mendelsches Bruderbuch from 1395). During the 16th century, the lathe was adapted for the production of screws, and throughout the 17th century, it acquired extra tooling, such as cams and patterns, which enabled repetitive machining of relatively complicated shapes. 
Eventually, after a relatively slow development of machine tools, the Industrial Revolution, which lasted from approximately 1760 to 1840 , blossomed and gave rise to a diverse variety of new machine tool designs. It was the first-ever large-scale transition to new manufacturing processes. Hand production methods were replaced by steam-driven machinery. Due to significantly higher power, most of the industrial lathes were made out of metal. In addition to the basic machine tools (the lathe, drilling machine, etc.), more elaborate machine design variations appeared. The second and third Industrial Revolutions followed. New technologies powered production machines with electricity and automation, but the nature of production remained much the same. For thousands of years, manufacturing technologies were mostly focused on the processing of larger pieces into smaller ones by applying different cutting tools. It was considered a holy grail to use the exact amount of material without having any scrap left over. Some technologies, such as forging and casting, were close to reaching this goal, but they could not produce very complex parts or reach the process flexibility, tooling cost level, lead time, and automation level required in the modern era of mass customization.

According to Groover , manufacturing involves a combination of machinery, tools, power and labor, as shown in Figure 3. Historical technological developments focused mainly on increasing the level of support (tools, machines) to make any shape in any material while minimizing waste (energy and material) and the human effort required. The role of the human also began to change at this time. When we consider early stone tools, the maker, the designer and the user were all one person. When crafts began to emerge (carpentry, for example), a separation formed, as the user was not necessarily the designer or maker of the item. As a result, production processes became highly complex and very much optimized.

Figure 3: Manufacturing defined as a technical process (Groover, 2013)

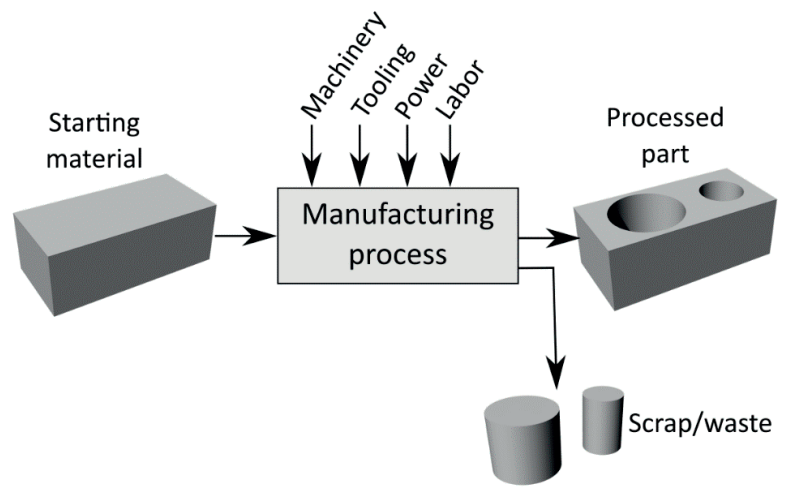


The ultimate goal of all technical advances is to improve all elements of the manufacturing process (machinery, tooling, power and labor). In the 1980s, thanks to advances in material science, 3D modeling and computational power, additive manufacturing became possible. This promised major advancements by using simple machines to produce very complex parts while keeping labor intensity and scrap production low. In 1981, Hideo Kodama from Nagoya Municipal Industrial Research Institute exploited the photo-hardening of some polymers under UV exposure. He used a mask pattern and scanning controls to solidify liquid polymer in the selected areas to fabricate a three-dimensional plastic model. Then, in 1983, Charles "Chuck" Hull invented stereolithography, the very first true additive technology that did not require any additional tooling, like masks. The new feature was a laser beam that scanned through liquid resin layer by layer to harden the material in particular spots. Since the 1980 s, many different additive technologies have been developed. They vary based on feedstock material and material form used (liquid polymer, plastic filament, plastic powder, metal powder, etc.), binding process (UV solidification, laser melting, laser sintering, gluing, etc.), machine architecture and build process (extrusion, blasting, powder bed, spraying, etc.). Additive manufacturing is unique in that it potentially allows the production of highly complex industrial products that require minimal human skill and effort with virtually zero waste. Moreover, the need for different tooling equipment, like fixtures, could be eliminated in some additive processes. These unique features of additive manufacturing differentiate it from other incumbent technologies.

\subsection{THE GENERIC AM PROCESS}

Despite the variety of the existing technologies, the ways in which AM processes are handled are similar. AM starts with a virtual CAD description that undergoes different manipulations to result in the physical part. Gibson (2015) identified eight key steps in AM, as shown in Figure 4:

\section{Conceptualization and CAD}

2. Conversion to STL/AMF

3. Transfer and manipulation of STL/AMF file on an AM machine

4. Machine setup

5. Build

6. Part removal and clean-up

7. Post-processing of part

8. Application

According to Gibson (2015), different products may involve AM in different ways and to different degrees. Part size and complexity may influence the extent to which AM stages are applied. Furthermore, the early stages of the product development process may only require rough parts, with AM being used because of the speed at which it allows parts to be fabricated. At later stages of the process, parts may require careful cleaning and post-processing, such as sanding, surface preparation and painting. 
Figure 4: Eight generic stages of AM process (Gibson, 2015)

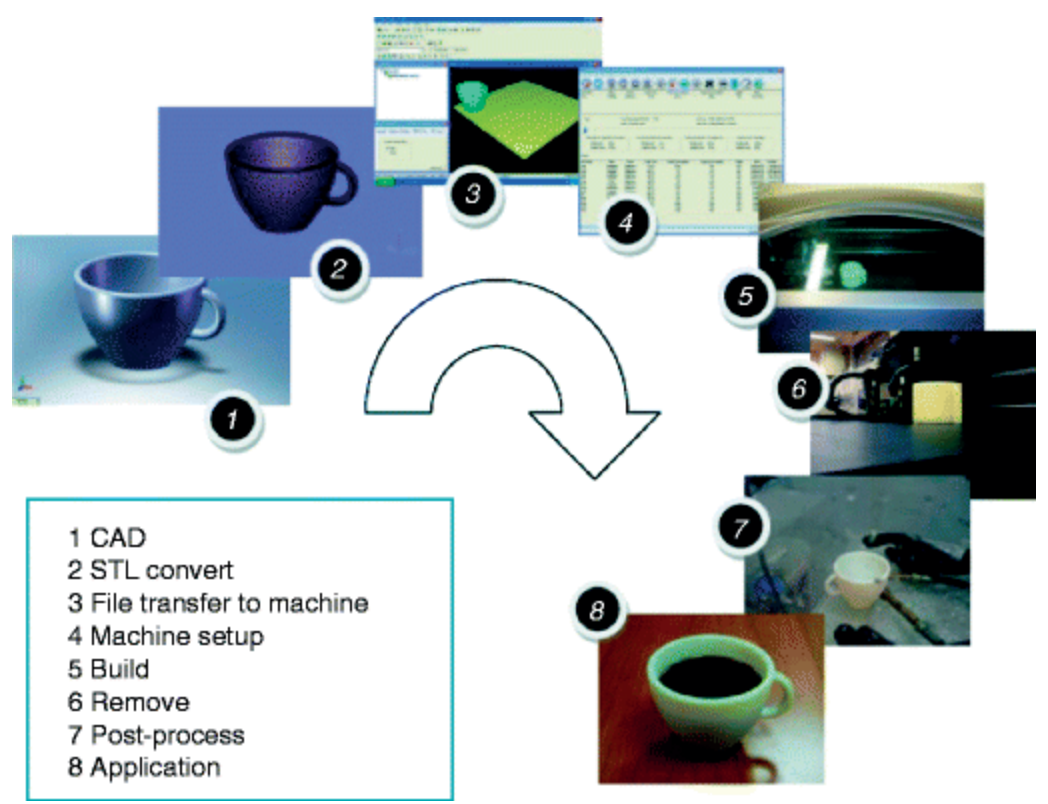

\subsubsection{Step 1: CAD}

According to Gibson (2015), all AM parts must start from a software model that fully describes the external geometry. This can involve the use of almost any professional CAD solid modeling software, but the output must be a 3D solid or surface representation. Reverse engineering equipment (e.g., laser and optical scanning) can also be used to create this representation. Internal geometry, which is called a lattice structure, is typically defined at the Step 3 together with other build parameters. Some new AM software applications require this internal geometry to be described by the CAD system.

\subsubsection{Step 2: Conversion to STL}

$C A D$ files store geometry in their native standard (geometry and design history) or in dumb solids (just geometry, for example iges and step). To transfer geometry between CAD programs to print software an approximation of the geometry by surface triangles is used. This format is understood by all printers while other formats exist with their own benefits and drawbacks. In general these alternative formats are less well supported by printing software. Nowadays, an STL file format describing the triangulated mesh surfaces has become the de facto standard in AM. Solid CAM models are converted to STL format as the basis for the calculation of the slices. 


\subsubsection{Step 3: Transfer to AM machine and STL file manipulation}

The STL file describing the part must be transferred to the AM machine. There may be some general manipulations for the correct size, position and orientation of the part. In cases where multiple parts can be created together, the design of the build volume and packing must be defined in this step as well.

\subsubsection{Step 4: Machine setup}

The AM machine must be properly set up prior to the build process. Settings like material constraints, energy source, layer thickness, timings, etc. are related to the build parameters. All products in the build area will be split in horizontal slices, where the machine will create these products slice after slice.

\subsubsection{Step 5: Build}

Building the part is mainly an automated process, and the machine can largely carry on without supervision. At this stage, only superficial monitoring of the machine is required to ensure no errors have occurred, like running out of material, losing power, software glitches, etc.

\subsubsection{Step 6: Removal}

Once the AM machine has completed the build, the parts must be removed. This may require interaction with the machine, and there are usually safety interlocks to ensure, for example, that the operating temperature is sufficiently low or that there are no actively moving parts.

\subsubsection{Step 7: Post-processing}

Once removed from the machine, parts may require some additional clean-up before they are ready for use. Parts may need further surface treatment to reach a particular surface roughness or they may have supporting features that must be removed. Therefore, this step often requires time and careful, experienced manual manipulation. The focus of this work aims to address this step by reducing and eliminating support structures and improving the surface roughness.

\subsubsection{Step 8: Application}

The parts should now be mostly ready for use. However, they may still require additional treatment before they are acceptable. For example, they may require priming and painting to create an acceptable surface texture and finish. These treatments can be laborious and lengthy if the finishing standards are very high. They may also need to be assembled with other mechanical or electronic components to form a final model or product. Additionally, quality inspection and certification could be required. 


\subsection{OVERVIEW OF MAIN AM PROCESS GROUPS}

Additive manufacturing (AM) is becoming increasingly important in the industry and for generic applications. For instance, fused deposition modeling (FDM) has gained significant momentum and penetrated both the business and consumer markets. Although FDM is just one of many AM processes, in the consumer market, the term "3D printing" is often incorrectly used as a synonym for AM. Figure 5 shows how the FDM process creates solid parts. Solid filament is fed through the heating element to the nozzle, where the filament becomes molten and is extruded to create three-dimensional objects. After expiring Stratasys' patent US5121329A apparatus and method for creating three-dimensional objects, which describes a fused deposition, many companies began to offer cheap hardware, which sparked much interest in additive manufacturing. Beyond the above-given well-known example of FDM, there are many AM processes that share common characteristics like layer by layer construction of geometry, a material deposition and a material consolidation step. But they all have their own unique methods for these steps and have their own advantages and disadvantages.

Figure 5: A typical FDM setup (ModuleWorks)

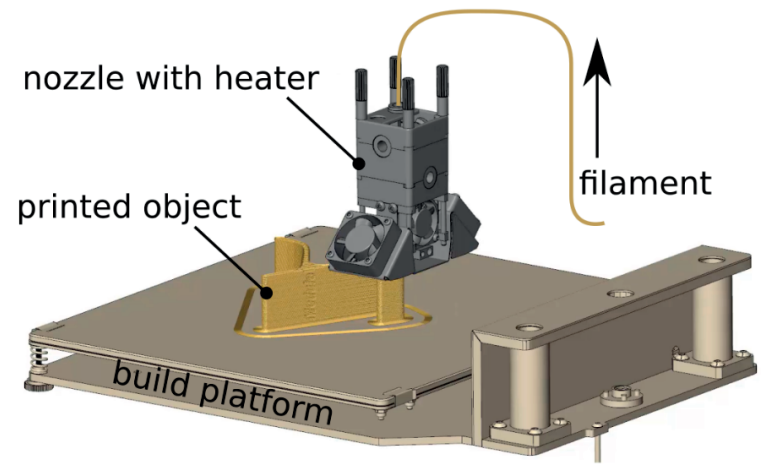

AM processes differ in the material used as feedstock, energy source, and the way in which the material is deposited. They have been grouped in 7 main AM process groups based on some common characteristics (mostly not material or energy source). ISO (the International Organization for Standardization) has developed the ISO/ASTM 52900:2015 standard, which defines the general principles and terminology for AM. According to ISO/ASTM 52900:2015, AM technologies can fall into one of seven process categories. The following sections provide detailed explanations of these various AM process groups, within these groups there are many different processes and process variants. 


\subsubsection{Material extrusion}

According to ISO/ASTM 52900:2015, material extrusion is an additive manufacturing process in which material is selectively dispensed through a nozzle or orifice. The material is deposited on demand and in most material extrusion processes no unused material is present on the build space. Molten or viscous material is extruded at precise locations, where it solidifies. The material should be viscous in the nozzle but have enough stiffness after deposition to hold its shape and carry subsequent layers.

FDM is the best-known extrusion-based technique. Thermoplastic filaments are the most frequently used material. These often include ABS, PLA, and PEEK for functional applications. The filament matrix can be enriched with fibers, ceramics and metals. As shown in Figure 6, filament is fed into the extrusion head, which is equipped with a heater and a nozzle. Then, the molten feedstock is deposited. The first layer is deposited on the build platform. The next layers are built upon the previously deposited material and support structures must be built for overhanging surfaces.

The direct ink writing technique ejects liquid ink, material droplets, or conductive pastes onto a substrate. Electronic components can be manufactured with this technology. Robocasting is a technique for dense ceramics and composites that is based on a layer-wise deposition of highly loaded colloidal slurries. Printing of Beton and clay is also possible and $3 \mathrm{~d}$ printing method allowing to build constructions at an architectural scale is called "contour crafting".

Metal parts can also be fabricated indirectly with the extrusion methods. First, the green parts are formed from the metal composite or slurry. No mold is needed. Support structures can be formed from ceramics for easy detachment afterward. Second, the binder is washed away. Then, the part must be sintered in a furnace. Because of this sintering, the parts shrink and may also warp. The part surfaces may require further finishing. Figure 7 shows several metal FDM parts. 
Figure 6: Fused deposition modeling (FDM) setup (3D Hubs, 2018)
(1) Filament spools
(2) Main filament
(3) Support filament
(4) Extrusion head
(5) Printed part
(6) Support structure
(7) Build platform

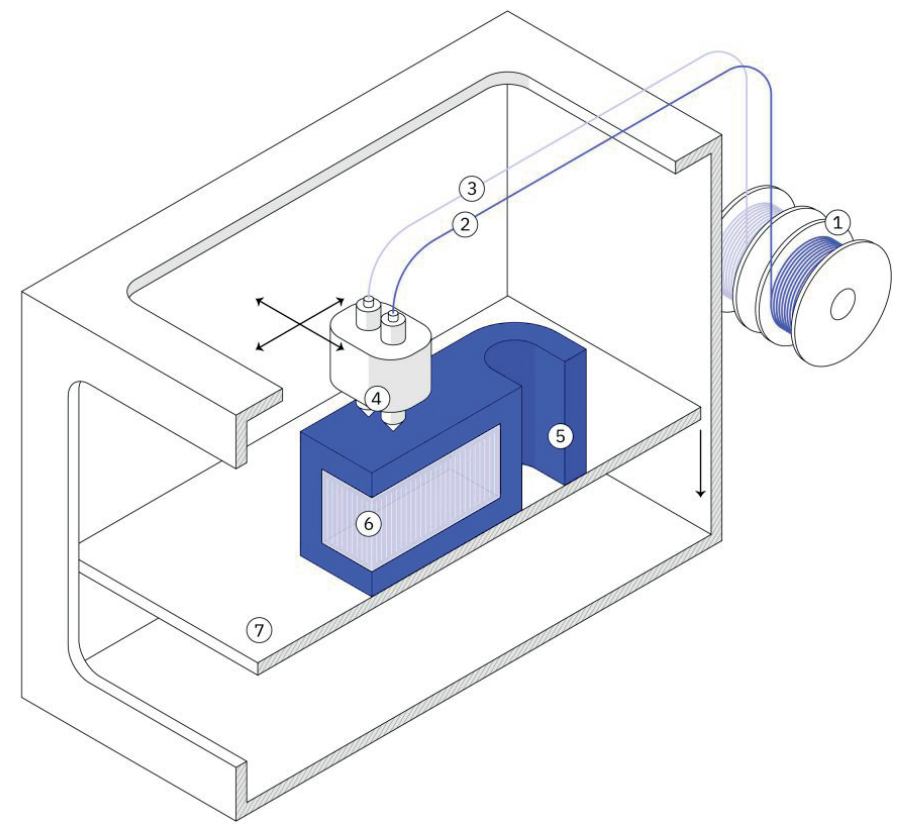

Figure 7: Examples of metal FDM parts (Masood \& Song, 2004)

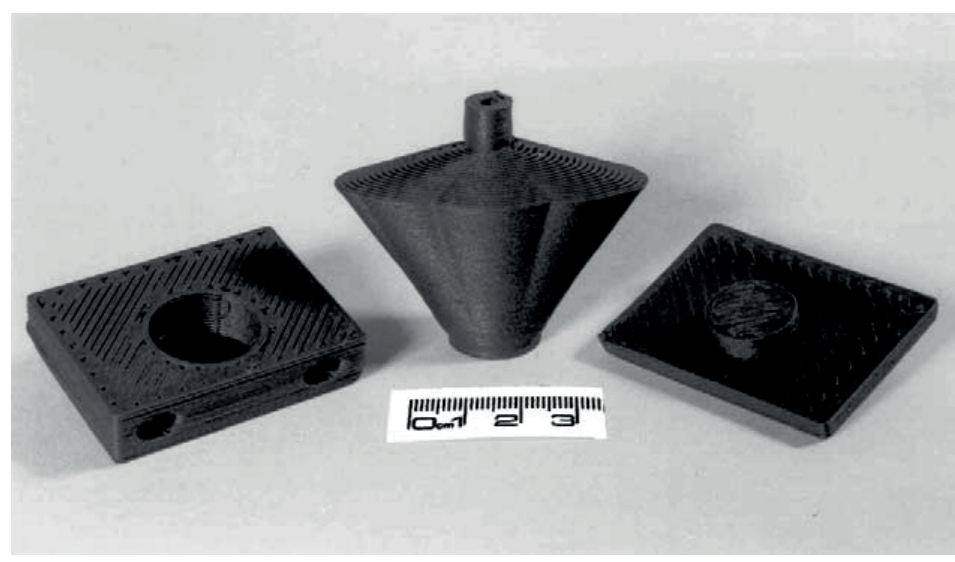




\subsubsection{Powder bed fusion}

According to ISO/ASTM 52900:2015, powder bed fusion (PBF) is an additive manufacturing process in which thermal energy selectively fuses regions of a powder bed (a build area). Every layer completely covers the powder bed with powder. After scanning with a thermal energy beam, the powder bed lowers and the process repeats until the part is made. The material fills the entire build space. This process transforms part of the material as the unused material may support the upper layers.

Powder bed fusion includes the following commonly used printing techniques: direct metal laser sintering (DMLS), electron beam melting (EBM), selective heat sintering (SHS), laser powder bed fusion (LPBF) and selective laser sintering (SLS).

LPBF is a process that utilizes a laser to melt thin layers of metal powder. As shown in Figure 8 , the laser scans the top layer of the powder bed and fuses powder particles to the already molten material. Although the last powder layer is always supported by the powder from the previous layer, support structures should also be fabricated. These support structures transfer any excess to the build plate and hold the workpiece in place to prevent the part from warping. When complete, these support structures must be removed, which is a challenging task. With this process, the part dimensions cannot exceed the size of the powder bed. It is impossible to manufacture a fully enclosed, hollow object because the powder will become trapped inside. Additionally, LPBF parts shrink after cooling down, leading to even more warping. The cost of recycling or throwing out powder is another important consideration. LPBF is compatible with atomized materials that include copper, aluminum, stainless steel, cobalt chrome, titanium and tungsten alloys. It is often used for the most demanding applications, such as medical, aerospace and automotive. The surface quality resulting from this technique may be insufficient, as shown in Figure 9, so additional machining may be needed. EBM differs from LPBF as an electron beam is used instead of a laser beam, where bed temperatures are higher and the process is held at higher temperatures, which result a different cooling time and a different set of materials DMLS uses a laser, but it sinters the metal powder instead of melting it. 
Figure 8: Direct metal laser sintering (DMLS) and laser powder bed fusion (LPBF) setup (3D Hubs, 2018)
(1) Laser
(2) $\mathrm{XY}$ scanning mirror
(3) Recoater
(4) Printed part
(5) Support structure
(6) Powder bed
(7) Overflow bin

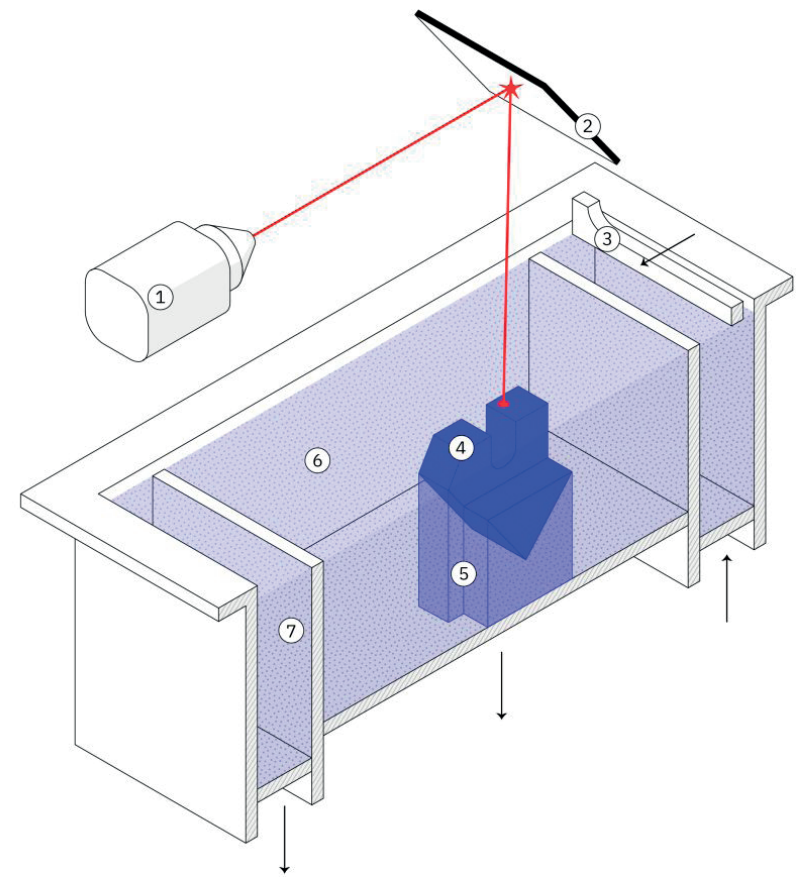

Figure 9: Examples of LPBF parts (McCue, et al., 2019)

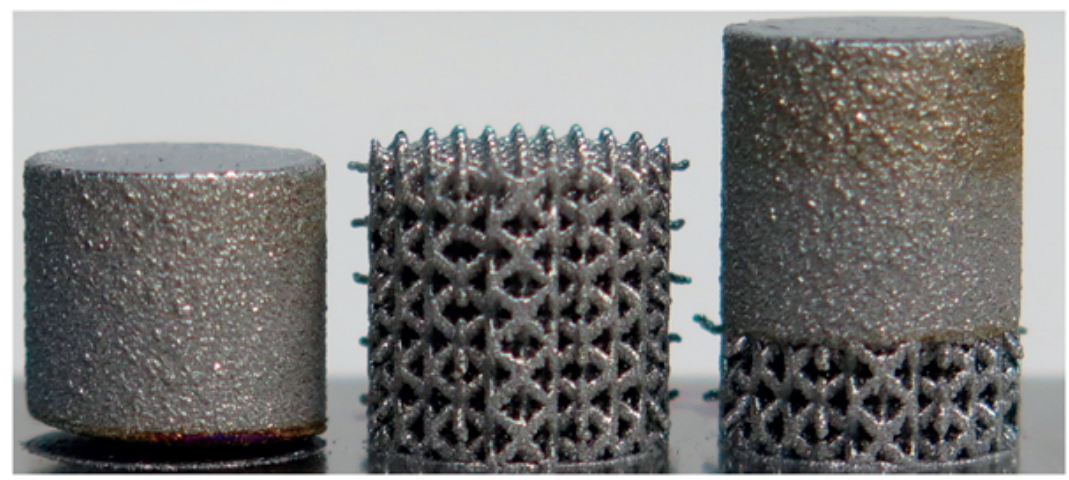

SLS uses a laser to sinter polymers and a thermal head to selectively fuse the polymer powder . Since thermal distortion of the part is not an issue, SLS and SHS require no support structures, as shown in Figure 10. SLS can be used for the rapid manufacturing of tooling, jigs and fixtures. 
Figure 10: Selective laser sintering (SLS) setup (3D Hubs, 2018)
(1) Laser
(2) $X Y$ scanning mirror
(3) Recoater
(4) Printed part
(5) Build platform
(6) Overflow bin

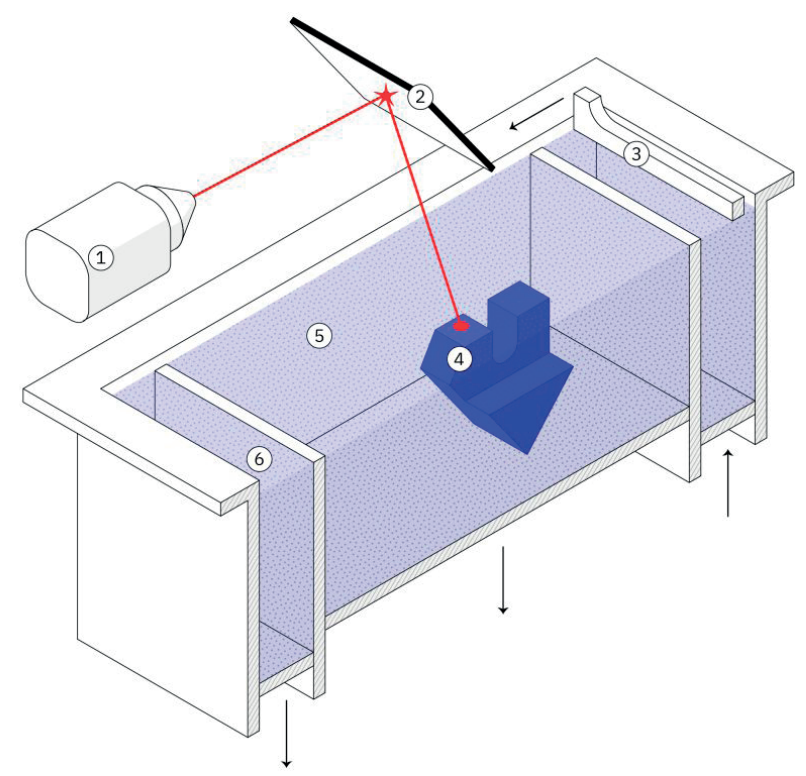

\subsubsection{Vat photopolymerization}

According to ISO/ASTM 52900:2015, vat photopolymerization is an additive manufacturing process in which liquid photopolymer in a vat is selectively cured by light-activated polymerization. When exposed to certain wavelengths of light, the liquid photopolymers' molecules rapidly bind together and cure into a solid state. The material fills the entire build space. This process transforms part of the material, so unused material cannot support the upper layers due to its properties.

Vat photopolymerization includes the following commonly used printing techniques: stereolithography (SLA), continuous liquid interface production (CLIP) and direct light processing (DLP).

SLA is the oldest and most known vat photopolymerization process. The mechanics of SLA is very similar to PBF, but instead of powder, SLA uses a liquid photopolymer resin. The laser scans the top layer to selectively solidify regions. Next, the build platform lowers and the new iteration repeats. Since each layer must be connected to the previous, support structures are unavoidable. 
DLP utilizes an array of mirrors (a DMD) and a single light source. Each mirror can be switched to two angles, of which only one is directed towards the resin. The DMD transforms a single light source to an array of light beams which selectively can be switched on or off. A digital light projector flashes a single layer all at once. There are two approaches to build the part. The first is a top-down approach, which is all about placing the light source below the vat. The vat has a glass bottom through which the light shines. When a new layer is built it stick to the previous layer and the vat bottom. Strategies are needed so the layer peels from the vat bottom, but not from the previous layer. The build platform moves upward and the final part is built upside down. The second is a bottom-up approach, which is all about placing the light source above the vat, so the printing platform is dipped in the vat.

SLA (Stereolithography) is similar to DLP where a UV laser is used with a single mirror instead of DMD, as presented in Figure 11.

Figure 11: Stereolithography (SLA) setup (3D Hubs, 2018)
(1) Build platform
(2) Support structure
(3) Printed part
(4) Liquid photopolymer
(5) Recoater
(6) Transparent screen
(7) X-Y scanning mirror
(8) UV laser

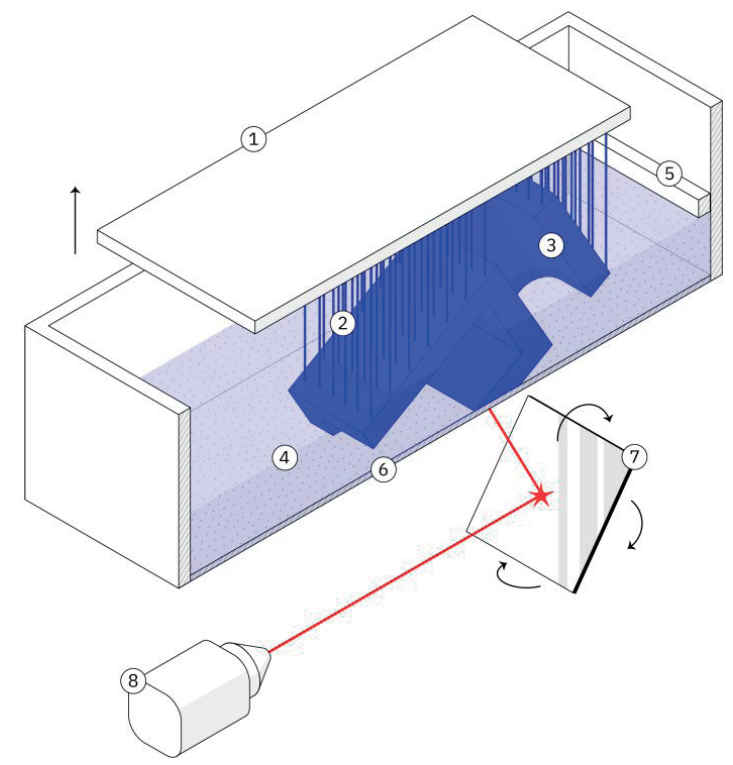

CLIP utilizes a source of constant light that illuminates the precise cross-section of the object through a UV-transparent and oxygen-permeable window. The object continuously rises, making the 3D printing process continuous as well. It is claimed that print speeds must increase by at least an order of magnitude while maintaining excellent part accuracy. CLIP has coated the glass window so a barrier layer exists between the layer being formed and the glass window. Because the layer does not stick anymore, production speeds can go up. 
SGC utilizes a source of UV light along with a mask. This means light that passes through the mask cures the entire layer.

Given the nature of the process, only photodegradable substances consisting of light-sensitive polymerized monomer/oligomer molecules can be used. This limitation restricts the use of some materials. As the process uses liquid, support structures are often needed. In addition to the removal of supports, the post-processing procedure requires the removal of all residual resin using a bath of isopropyl alcohol. Further postprocessing step is UV curing to make the parts stronger.

Due to the high print quality and relatively low mechanical properties, vat photopolymerized parts are usually used in jewelry or other artistic endeavors, such as castable models. The production of dental models and hearing aids is another common application. The top down approach can be used for larger parts (up to meters in length) with good surface properties and small details, while the bottom up method is limited to smaller products.

\subsubsection{Material jetting}

According to ISO/ASTM 52900:2015, material jetting (MJ) is an additive manufacturing process in which droplets of build material are selectively deposited. Because the photopolymer resin is sprayed in droplets before it is solidified, $\mathrm{MJ}$ is often compared to the $2 \mathrm{D}$ inkjet process. Material jetting processes require supports, which are often made from a dissolvable material. The material is deposited on demand and no unused material except supports is present on the build space.

Standard drop-on-demand MJ deposits droplets of wax and dissolvable support material, where a set of thermoset photopolymer resins that are similar to those used in SLA in terms of properties and limitations can also be used. Nowadays, MJ is usually associated with the jetting of tiny drops of photopolymer, which are then cured (solidified) by a UV light, as shown in Figure 12. It is highly accurate and yields a smooth surface finish. It can execute full-color, multi-material printing, as the printing head can incorporate multiple nozzles, each depositing different materials and/or different colors in a single printing process. Although support structures are required, these can be easily dissolved without marking the surface. However, due to the restricted use of photopolymers and wax, the fabricated objects are often weak and unusable for load-bearing and functional applications.

MJ can also build parts using metal and ceramics. Metal droplets solidify by cooling down. The Vader system melts the aluminum and shoots out droplets using electromagnetic pulsing, as shown in Figure 13. The company XJet developed a so-called "NanoParticle Jetting" process, which uses a liquid that contains building nanoparticles or support nanoparticles. High temperatures inside the build envelope cause the liquid to evaporate, leaving behind only the parts made from the building material. This technique is suitable for metals and 
ceramics, but it requires further sintering, like metal FDM. Usually, metal MJ is not as fast and accurate as polymer MJ.

Another option is turning very fine droplets into an aerosol. Aerosol jet printing is often used to deposit silver nanoparticle ink on electronic components to act as electric power or signal track.

Figure 12: Material jetting (MJ) setup (3D Hubs, 2018)
(1) Material container
(2) Inkjet print head
(3) UV curing light
(4) Printed part
(5) Support structure
(6) Build platform

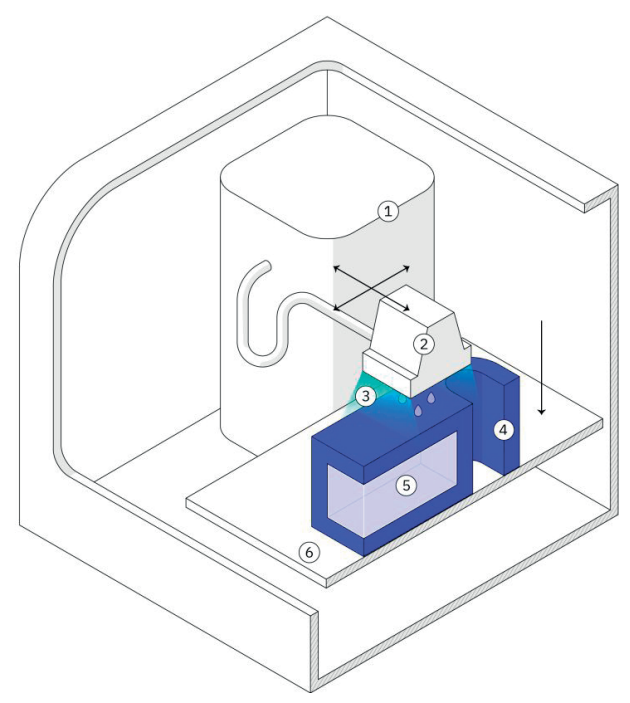

Figure 13: Vader metal printing system (Elliott, 2019)

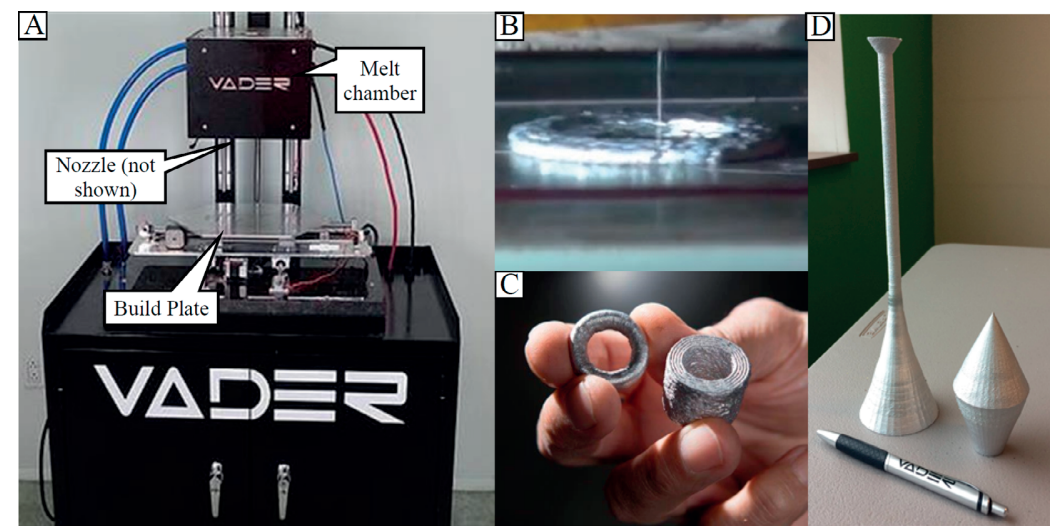

a) Vader Systems platform, b) image of molten aluminum deposition, c) and d) printed parts 


\subsubsection{Binder jetting}

According to ISO/ASTM 52900:2015, binder jetting (BJ) is an additive manufacturing process in which a liquid bonding agent is selectively deposited to join powder materials. The material fills the entire build space. The process transforms part of the material and unused material may support upper layers.

The kinematics of BJ is similar to that of SLS. With every iteration, a layer of powder material is deposited on the powder bed. Then, droplets of adhesive are ejected by an inkjet printhead to selectively bind the powder particles together, as shown in Figure 14. There are no support structures, as the printed object is being self-supported within the powder bed.

The powder materials are usually gypsum, sand, ceramics, various types of metal alloys (steel, titanium, Inconel 625 and copper) or thermoplastic polymers and polyamides in granular forms. The color may be added at the same time as the binder, enabling full-color fabrication.

Binder jetting is a relatively fast and cheap technology and parts can be printed in full color. However, without additional treatment, the parts have limited mechanical characteristics. To improve mechanical properties, additional curing in an oven, lasting up to several hours, might be required. Sand binder jetting is mostly used to produce casting patterns for dies and molds and full-color prototype models.

For metal BJ, sintering is required at higher temperatures to reduce porosity, but this may lead to shrinkage. Optionally, metal parts can be polished and plated. Metal binder jetting is useful to fabricate unique aesthetic and functional metal parts with a good surface finish. Compared to powder bed fusion techniques, the binder jetting method has the advantage of not employing heat during the build process, which prevents the creation of residual stresses in the parts. Figure 15 shows an example of a "green" binder jetted part made of stainless steel. 
- Figure 14: Binder jetting (BJ) setup (3D Hubs, 2018)
(1) Material container
(2) Inkjet print head
(3) Recoater
(4) Printed part
(5) Powder bed
(6) Overflow bin

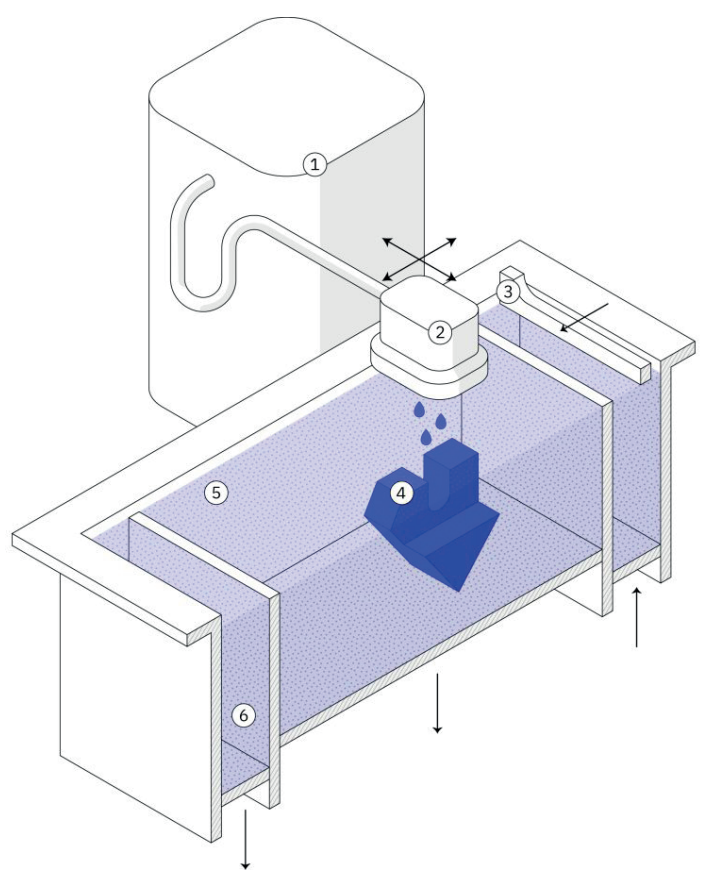

Figure 15: Raw binder jetting stainless steel part (Elliott, 2019)

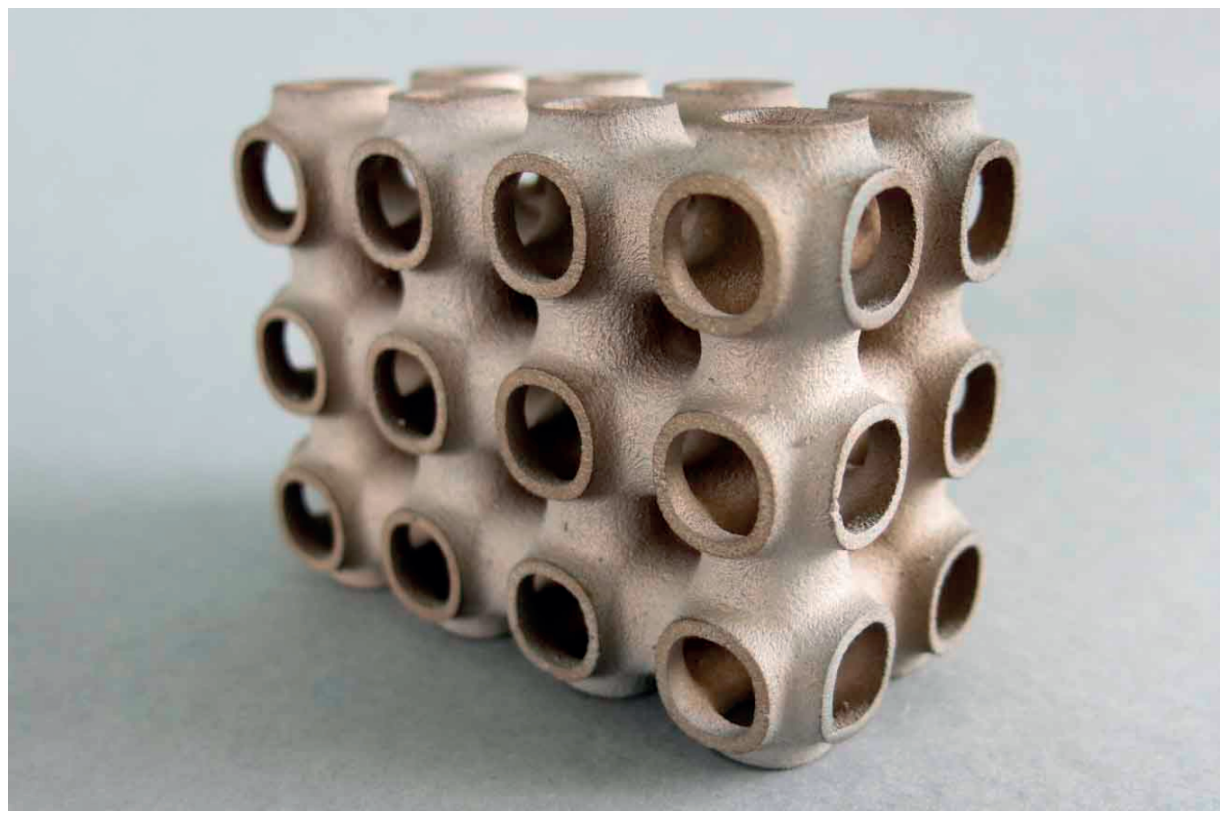




\subsubsection{Sheet lamination}

According to ISO/ASTM 52900:2015, sheet lamination (SL) is an additive manufacturing process in which sheets of material are bonded to form a part. The material fills the entire build space. The unused material is cut from the sheets but is not removed to support the next layers.

The sheet lamination (SL) 3D printing and manufacturing technique, also known as laminated object manufacturing (LOM), consists of super-positioning several layers of material composed of foil to manufacture an object. Each foil is cut to shape with a knife or a laser to fit to the object's cross-section. Stacking them layer by layer, the sheets are joined together and 2D profiles are cut from the last sheet. Finally, any unnecessary cut profiles are removed, as shown in Figure 16. Bonding between these layers can be achieved in several ways: (a) gluing or adhesive bonding, (b) thermal bonding processes, (c) clamping, and (d) ultrasonic welding.

SL technology can produce parts made from polyvinyl chloride, carbon fiber, thermoplastic, paper, and metal sheets. The advantages of this technology include its speed, low cost and the ease of material handling. Furthermore, large models can be built easily and some materials can be colored. However, there are several limitations, including the poor quality of complex surfaces and the difficulty of connecting layers. Its usability for functional prototypes is limited. Support removal is a problem in many cases because it should be done manually. Sheet lamination is used mainly for the rapid prototyping of scaled models and conceptual prototypes that can be tested for form or design. It can also be used to make sand-molded and metal casting.

Ultrasonic Additive Manufacturing (UAM) is a variation of SL in which thin metal sheet strips (as thin as $\sim 0.125 \mathrm{~mm}$ ) are joined together using ultrasonic vibration. Due to very local heat input, it allows encapsulation of electronic components in a metal housing as shown in Figure 17. 
Figure 16: Laminated object manufacturing (LOM) setup (ManufacturingGuide, n.d.)

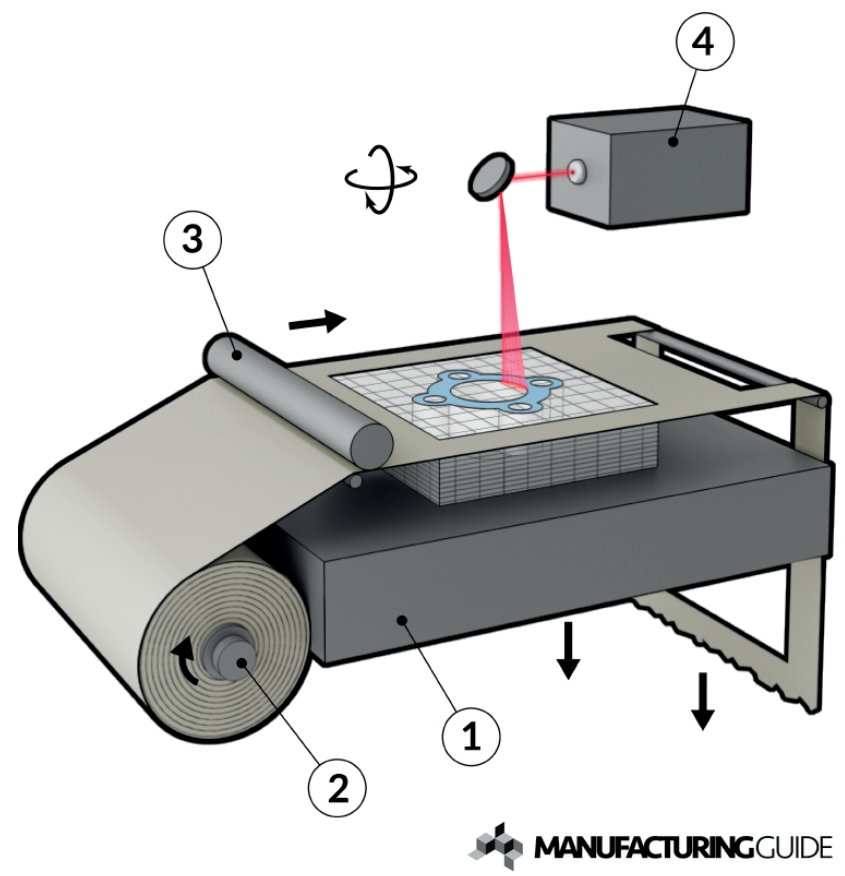

Figure 17: A metal part fabricated with the Fabrisonic ultrasonic additive manufacturing process (Clarke, 2017)

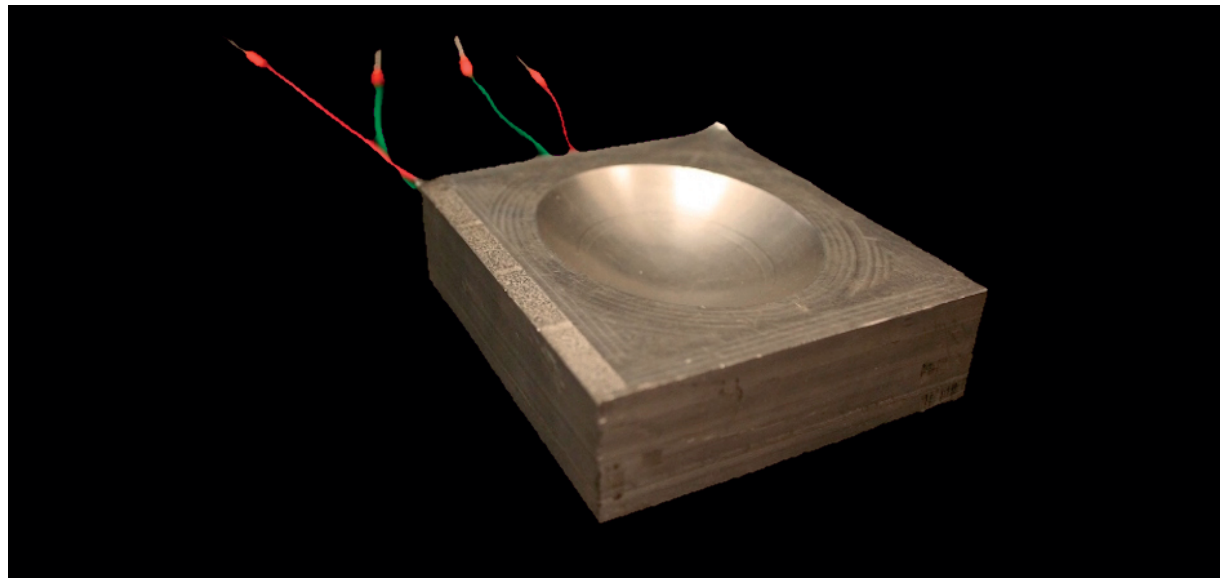




\subsubsection{Directed-energy deposition}

According to ISO/ASTM 52900:2015, directed-energy deposition (DED) is an additive manufacturing process in which focused thermal energy is used to fuse materials by melting them as they are being deposited. "Focused thermal energy" means that a highly focused energy source (e.g., a laser, electron beam or plasma arc) is used to melt the materials being deposited. The material is deposited on demand and no unused material is present on the build space.

A typical DED machine consists of a nozzle mounted on a multi-axis arm, which delivers the feedstock material (usually metal powder or wire) onto the specified surface, where it is then melted by focused thermal energy, as shown in Figure 18.

Figure 18: Directed-energy deposition (DED) setup

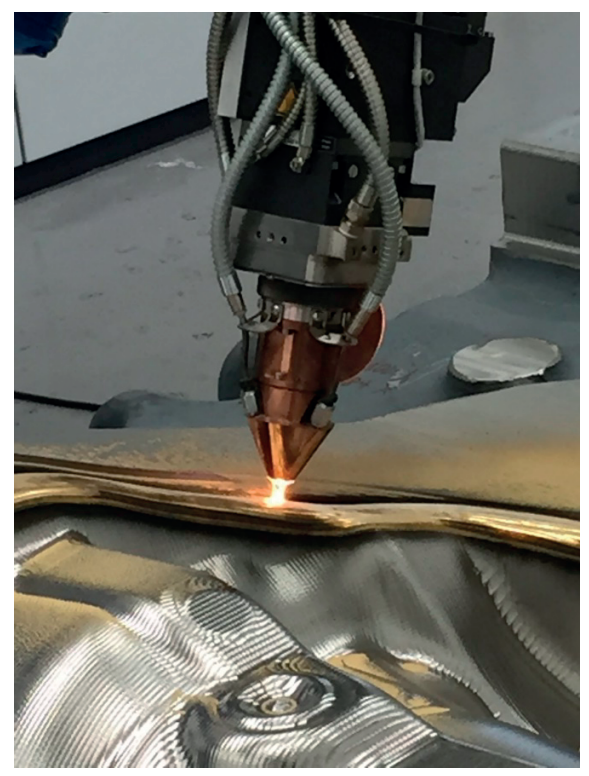

DED encompasses several different technologies. They distinguish themselves by the way the material is fused. There are three sources of thermal energy to fuse the feedstock: laser, electron beam and wire arc. Accordingly, there are three technologies: laser deposition welding, electron beam additive manufacturing, and wire arc additive manufacturing. Each one is best suited to different and specific purposes. Commercial names may differ, though.

The range of available supply metals is broad, including alloys of steel, titanium, nickel, copper, cobalt and zirconium. Moreover, tungsten, niobium and molybdenum can be used, as can ceramics and carbon fiber filament impregnated with a thermoplastic polymer matrix. Material 
deposition rates range from 3 to $9 \mathrm{~kg}$ of metal per hour. Compatible metals include titanium, tantalum and nickel.

A five-axis process may produce or repair complicated geometries without support. However, the surface finish and tolerances are often inappropriate and require additional post-processing to remove excess material. The main limitations of DED processes are their poor resolution and surface finish .

\subsubsection{Types of the AM processes}

Although there are seven different groups of AM processes, all AM processes can be distinguished into two types. The types depend on whether the material is deposited on an exact location on demand, or a full layer is deposited and locally processed to form a part:

1. Full-layer processes (binder jetting, PBF, sheet lamination, and VAT polymerization).

2. Exact deposition processes (material extrusion, material jetting and DED).

On the one hand, full-layer processes work across the entire layer to form a partial cross-section. Processes with solid material require no support structures since the lower layers can support the upper layers. However, the amount of material required to run the process may be significantly greater than the part volume. Recycling the used feedstock is also essential.

On the other hand, exact deposition processes require less feedstock than full-layer processes and multi-material is also possible. The major disadvantage is the need for support structures for overhanging geometries.

There is a growing interest in both types of processes. Those without support structures are easy to implement and use "one button" equipment. FDM has also drawn significant attention, especially FDM with PEEK. PEEK material has strong mechanical characteristics and biocompatibility that enable its use in medical and industrial applications. However, support structures in PEEK setups are undesirable because the removal of strong PEEK support structures is not easy. DED is also popular among industrial users as it allows the deposition of an exact amount of metals at a high deposition rate. However, this high deposition rate comes with high layer thickness and high surface roughness. There should be a compromise. Ideally, no post-machining should be done at all. To reach this goal, the use of support structures must be eliminated. 


\subsection{SOCIAL AND ECONOMIC RELEVANCE OF ADDITIVE MANUFACTURING}

Additive manufacturing is unique in nature. With a layer-by-layer approach, one can make complex geometries that no other process can. The popular belief is that the production process is fully automated, so anyone can make any complex geometry anywhere, anytime. No high levels of knowledge or skills are needed. New products and services, mostly supported by the availability of new technologies, can have significant social and economic relevance. Naturally, the social and economic effects are tightly interwoven to boost each other. Improvements in human wellbeing are reflected in the economic performance, and increased wealth and economic growth unlock better opportunities for the adoption of higher standards of living. Thus, additive manufacturing is a valuable technology for all of society. President Obama, in his speech in 2013, announced the deployment of three advanced manufacturing hubs because he believed that 3D printing had the potential to revolutionize the fabrication of almost everything and create new jobs. Apart from the numerous economic benefits, additive manufacturing can have a profound societal impact in many areas, such as customized healthcare products to improve population health and quality of life, reduced environmental impact for manufacturing sustainability, and a simplified supply chain to increase the efficiency and responsiveness in demand fulfillment. For mass customization and spare parts, it is seen as the future of production of these types of parts. Additionally, existing parts can be redesigned and upgraded with AM to create specific advantages, like a more lightweight design.

Additive manufacturing has already paved the way to market niches that embrace the development and production of products that have a social impact. There are several different frameworks to define and assess these social impacts, such as frameworks from the United Nations Environment Programme, the Global Reporting Initiative, and the International Association of Impact Assessment. In a broader context, the social impacts encompass people's lives, culture, community, political system, environment, health and wellbeing, personal and property rights, emotional wellbeing, among many other. New applications in medicine can already be identified as a driver of increased population wellbeing, which is associated with an increase in life expectancy and quality of life. The reduction of associated costs may cause a rise in life expectancy because of the potential to customize healthcare products (e.g., surgical and dental implants, etc.). Figure 19 shows an example of how hearing aids can be customized and fabricated to fit each individual ear. Moreover, additive manufacturing has already paved the way for mission-critical applications . In 2018, GE Additive reported that it had created 30,000 additively produced fuel nozzle tips for the LEAP engine, shown in Figure 20. The use of additive manufacturing allowed the number of parts in a single fuel nozzle tip to be reduced from about 20 pieces, which were previously welded together, to just one whole piece. The nozzle tip's weight was cut by about 25 percent, enabling a new engine design with 15 percent better fuel efficiency than conventional engine designs. 
- Figure 19: 3D printed customized hearing aids at Sonova Holding AG (Fadhli, 2019)

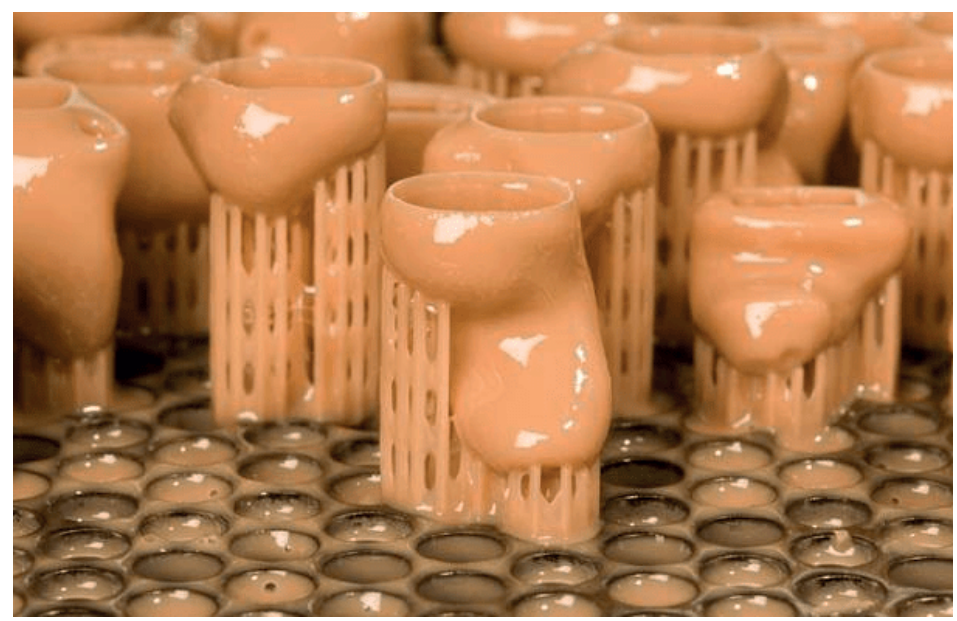

- Figure 20: GE additively produced fuel nozzle tips for the LEAP engine (GE Additive, 2018)

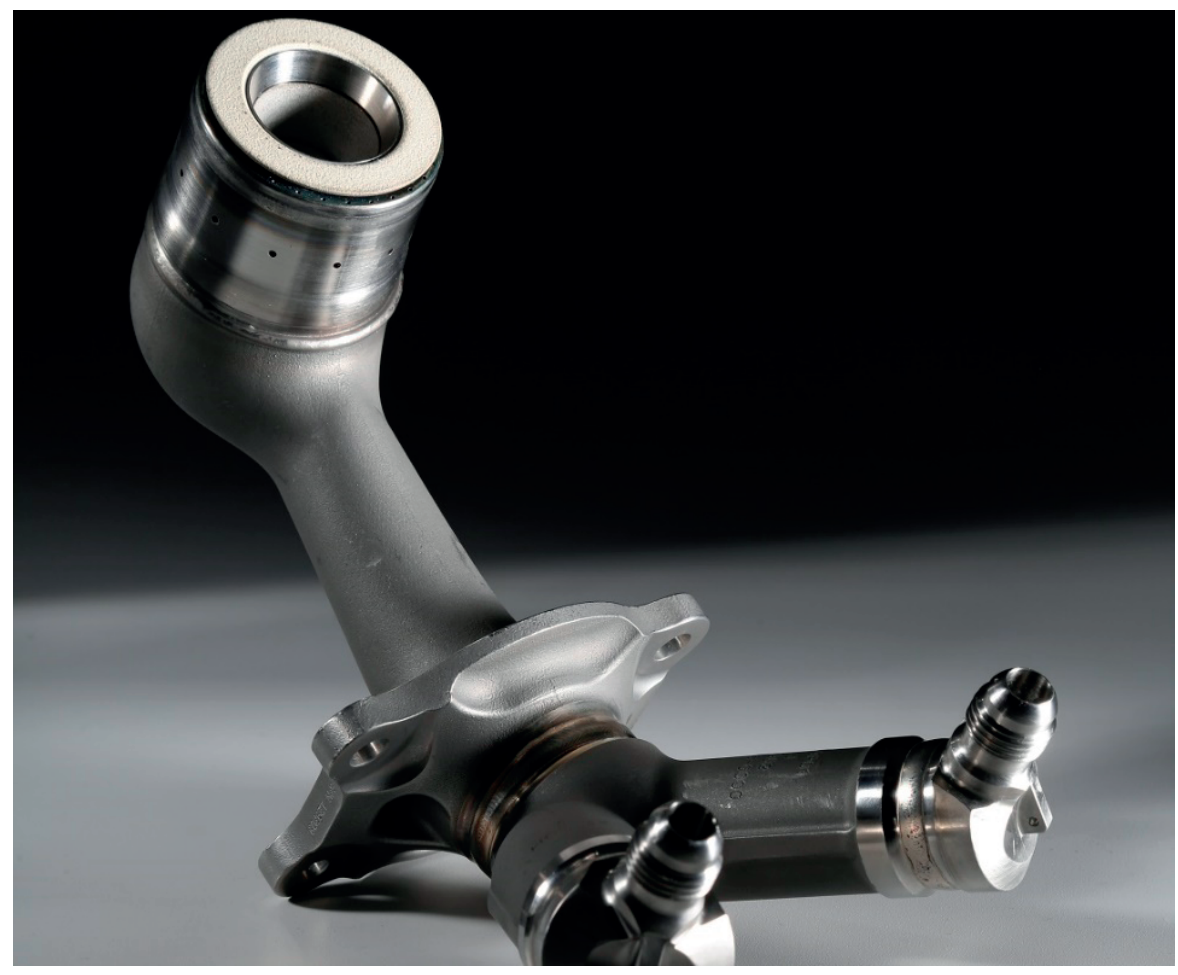


It is important to note that AM does not just offer positive outcomes. There are also some negative concerns. For example, there are multiple hazards regarding human health and the environment like titanium printing, FDM microparticles, toxic materials in SLA etc. Addressing these potential hazards will require an analysis of the fabrication methods. The powder-based fabrication methods usually use toxic metals, most of which cannot be metabolized by the human body. There are also risks associated with laser/ultraviolet exposure, shielding gas, and various resins and solvents. Efficient waste management is also an topic that must be closely regulated and managed to ensure additive manufacturing is part of an environmentally friendly strategy for our society. Apparently, the social impacts may also result in job losses, changes to the intensity of work, employment schemes and types of work, and the need to develop new skills. Additionally, weapons production can be named as one of the most concerning social impacts. The first blueprints for a 3D-printed handgun called "the Liberator" were released online in 2013 , allowing the untraceable fabrication and distribution of this deadly weapon.

In terms of significant social and economic impact on society of a new technology or product, a good example is the automobile, where there is a high and direct visibility of its impact by the consumers. It started to gain relevance around hundred years ago when mass deployment of this product to the market first began. Another good example is the smartphone, which emerged around ten years ago. The social and economic impact of both products, the automobile and the smartphone, on society is unquestionable. Increased mobility has facilitated the efficient transportation of goods and labor. Supply chains grew to a global scale, allowing closer cooperation between enterprises in different geographic markets. The number of smartphone users has now reached three billion. This huge user base of potential customers who are instantly available for different digital services has accelerated the shift toward a digital economy. As a result, many new jobs and market niches appeared to serve the effects of these paradigm changes. In 2018, mobile technologies and services generated 4.6 percent of GDP globally, a contribution that amounted to $\$ 3.9$ trillion of economic value added. The above examples illustrate what social and economic relevance means. Note, there are many products and services in the business-to-business marketplace that consumers typically do not see. Manufacturing technologies, processes and related machines and services have similar strong social and economic impact on society, but not similarly directly visible to the consumers. Manufacturing technologies are the key to building the many consumer products that we use every day. Introduction of home use $3 \mathrm{~d}$ printers to consumers increased in the recent years the visibility of AM process to consumers. A home $3 \mathrm{~d}$ printer makes this manufacturing equipment a consumer product. Looking at the exposure of this technology to consumers, there are just half a million shipments of 3D printers globally compared to 1.5 billion shipments of smartphones, a factor of 3000, which shows that consumers' exposure to 3D printers still has a long way to go. However, we should not underestimate the potential of the technology just because of the current numbers. Otherwise, one can fall into the trap of incorrectly evaluating newly launched technologies. For example, in 1980, McKinsey forecasted that by the year 2000 , there would only be 900,000 cell phone subscribers. The actual number was 109 million subscribers in the year 2000, which is about 120 times more than the 
original estimate. The most interesting aspect is the market growth rate. Similar to the exponential growth of cellular technology, the revenue of additive manufacturing products has also shown exponential growth in recent years, as depicted in Figure 21. Assuming a similar growth pattern, additive manufacturing will take a large share of the market.

Figure 21: Revenue for AM products and services worldwide (US\$ m) (Kingsley, 2018)

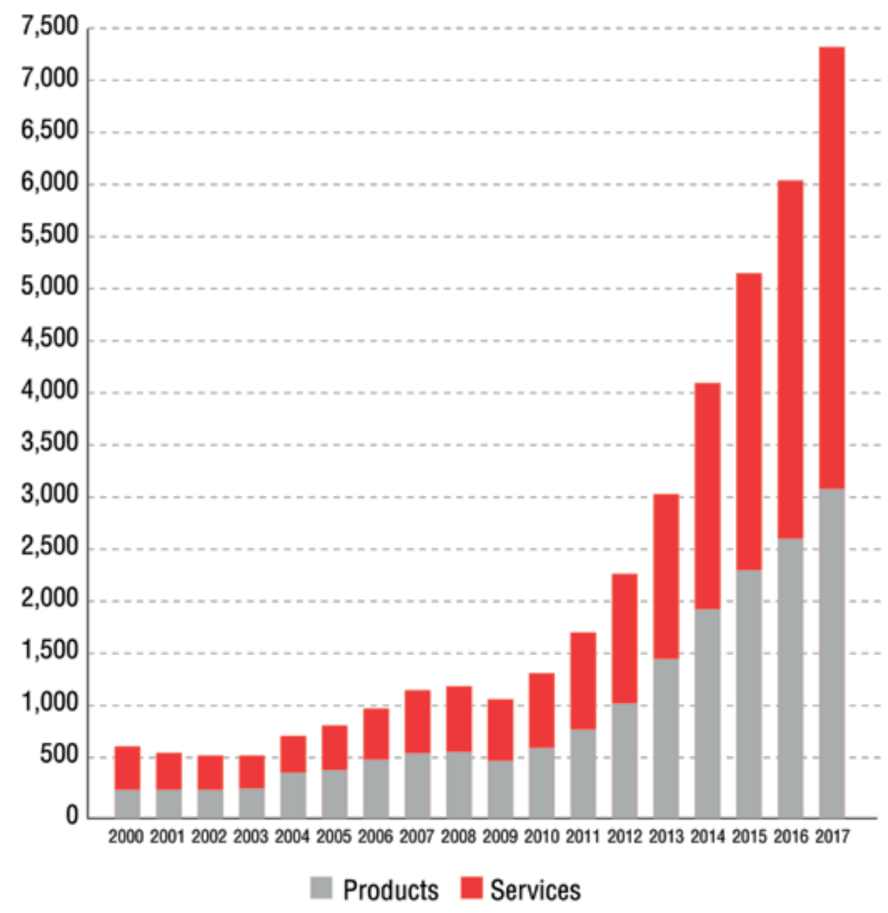

Today, manufacturing technology is dominated by subtractive methods that have significant social and economic relevance. Will additive manufacturing replace these subtractive methods in the coming years? Is the current size of the market for AM similar to that of the subtractive manufacturing market? Currently, the answer to both of these questions is no. Despite significant growth, in 2015, the additive manufacturing market represented just 0.04 percent of the global manufacturing market, with prototyping as the leading use . In a best-case scenario, AM will reach a level of market share where, thanks to the differences in technology, it can grow in a number of niche areas to drive down cost, improve designs and reduce manufacturing time. However, it has to be noted that applications in niche areas can indirectly trigger significant outcomes in a social and economic sense. Wohlers Associates, an agency that provides technical, market, and strategic advice on the new developments and trends in this field, 
predicted that, at most, it would account for 5 percent of the global manufacturing capacity, which would make 3D printing a $\$ 640$ billion industry .

In the context of additive manufacturing technology, engineers and researchers still have a lot of work to do. The possible improvements in reliability, ease-of-use, cost, speed and flexibility are all areas that deserve some attention. The sooner these improvements are made, the greater the deployment of this technology among other manufacturing technologies will be, and accordingly, this will lead to higher social and economic relevance.

\subsection{CHALLENGES IN PROCESS PLANNING FOR ADDITIVE MANUFACTURING}

Process planning in manufacturing is a preparatory step before manufacturing, which determines the sequence of operations or processes needed to produce a part. In AM, the movement of the machine and further process related parameters like deposition rate, temperature etc. are defined in process planning, and as such it has major impact on actual production of the part.

In the 1980s and 1990s, and again with the expiration of significant patents in the 2000s, AM was expected to replace subtractive machining, which did not happen. However, it did grow from a very small niche solution to a considerable economic size. The new fabrication concepts promised easy production of very complex parts without the complications that usually accompany conventional processes. Manufacturing of shapes with unlimited geometrical complexity was expected to become so trivial that re-fixturing and multi-axis toolpaths would not be required. However, after the initial hype and with broader and deeper investigation, researchers and industrial adopters realized that the traditional 2.5D approach (horizontal layer-by-layer deposition) had substantial limitations, including the need for support structures, insufficient surface quality on steep walls and overhangs, non-optimal structural performance in the build direction, volumetric distortions due to residual thermal stresses via direction change, and other issues. Many of these challenges are not relevant to process planning, like intellectual property management or the development and standardization of new materials. However, there are still several challenges , , , that could be resolvable, at least partly, by applying proper process planning algorithms. Traditional 2.5D approach in AM is utilizing existing process planning methods and its algorithms that define the movement of the machine, namely the path planning. To overcome the above-mentioned challenges in process planning, new algorithms for path planning and machines with bigger movement freedom can be used, which will be examined in this work.

\subsubsection{Challenge: elimination or optimization of the use of support structures}

As previously discussed, researchers realized that the traditional 2.5D approach (parallel slicing) had substantial limitations, such as the need for support structures for FDM, MJ and DED because surfaces cannot be printed in the air without being connected to previously printed 
material. There are other reasons to utilize supports in some AM processes, e.g. to solve thermal issues by allowing conduction of heat through support structures to control the thermal behavior during the process. The surface quality was also likely to be insufficient on steep walls and overhangs or there would be some other side effects, like non-smooth surfaces after removing the support structures, as shown in Figure 22. In addition, the costs of support removal would have a substantial effect on the fabrication costs. AM, however, was relatively new, and it became the topic of much research. It has since been proven in daily practice that for some geometries in some process variants it is hard to efficiently manufacture high-quality, functional products that do not need post-processing. Some of the specific challenges faced by those using AM also will be addressed in more detail in the remainder of this thesis.

Figure 22: Poor surface quality due to lack of supports (Kuhn, 2015)

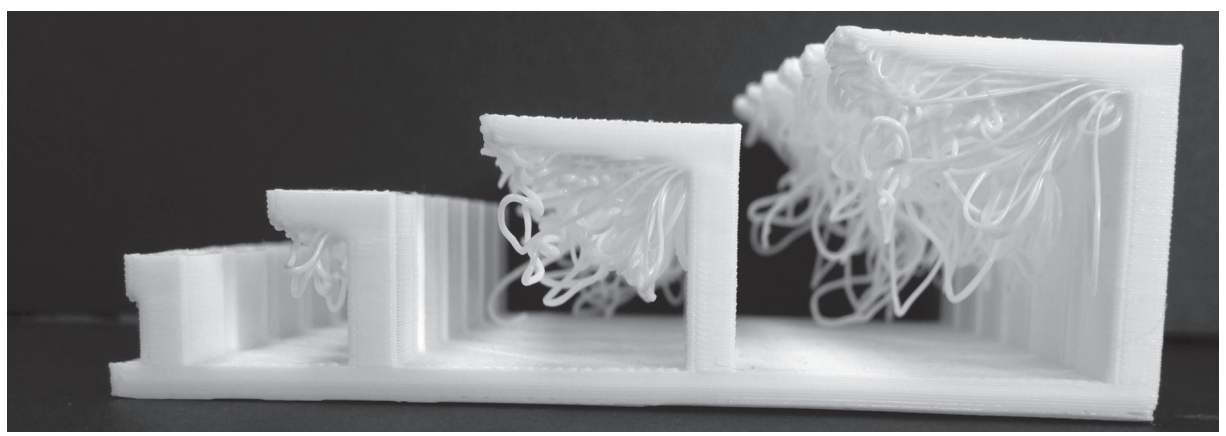

\subsubsection{Challenge: improvement of the part surface quality}

Improvement of surface quality is a significant challenge of most AM technologies. This surface quality deterioration may be due to the stair-stepping (also known as staircase) effect that arises from incrementally placing one layer on top of another, as well as insufficient printing resolution, which results in inaccuracies, particularly on curved surfaces. Figure 23 depicts how the staircase effect becomes more intense as the slices approach the top pole of a sphere. In horizontal areas and vertical walls, although staircase effect is not strong, there is still some surface roughness occurring due to the cross-section geometry of the deposition which is defined be nozzle size and layer height. 
Figure 23: 3D printed sphere with the explicit staircase effect around the pole (courtesy ModuleWorks $\mathrm{GmbH})$

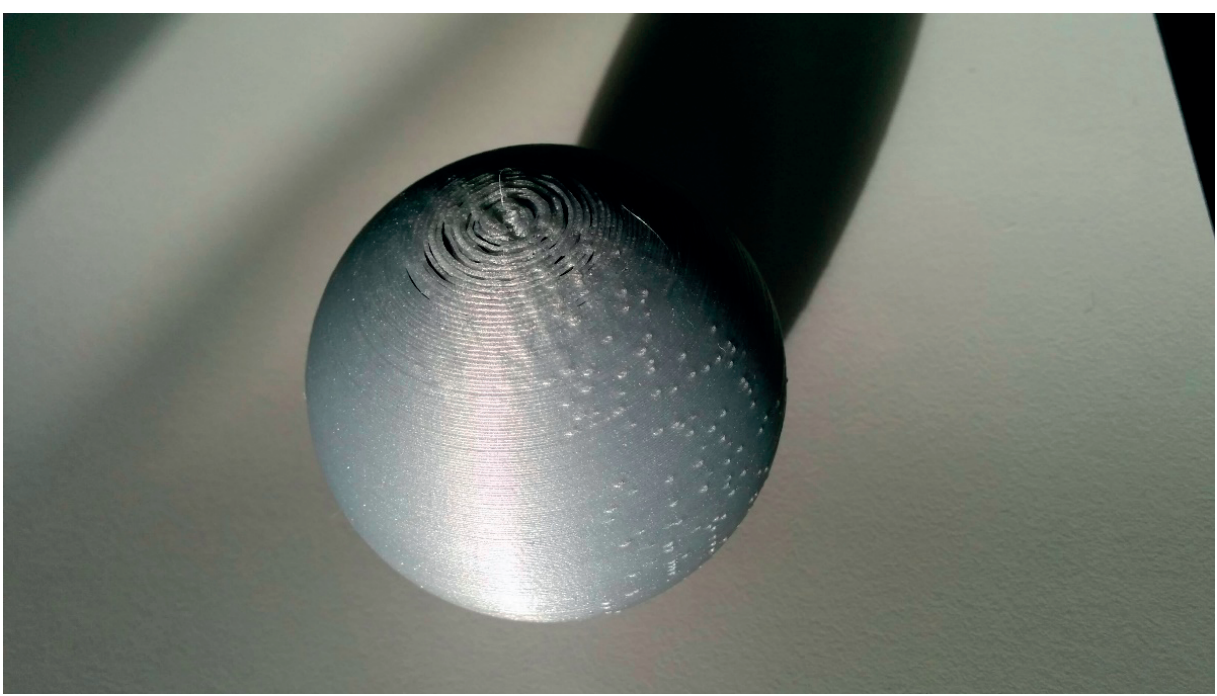

\subsubsection{Challenge and solution: development of an automated multi-axis AM process planning}

The challenges of support structures and surface quality (1.5.1 and 1.5.2) can be addressed by allowing the process to be driven by multi-axis kinematics hardware. Multi-axis AM offers many more possibilities in comparison with 2.5D AM. Figure 24 shows a five-axis AM process in which the nozzle has two additional rotational degrees of freedom to orient the nozzle. Reorienting of the nozzle during the deposition may resolve major issues by minimizing the stair-stepping effect and the need for support structures. Support material can now either be mechanical removed or dissolved (FDM). However, the support removal operation lacks automation and incurs additional costs. In the case of soluble FMD supports, support columns require about 75 percent more material because they are generally weaker compared to other materials and buckle easily under load. With mechanical removal, technicians use a manual grinding machine or manually chip away the supports. In addition to the time required, support removal leaves surface damage, so the object must then be sanded or polished to remove any remaining marks. It is theoretically possible to resolve these quality issues by applying additional degrees of freedom to the nozzle orientation and changing the path planning to be orthogonal to nozzle orientation such features of geometry can be built along their directions. This would also reduce the production time and costs because it would eliminate the need for support material and operations for support removal. It would also improve surface roughness because the toolpaths could be programmed to align with the part surface. Undoubtedly, it comes at some cost. 
Figure 24: Coating of a screw conveyor with five-axis DED at Fa. Huber-Tec GmbH

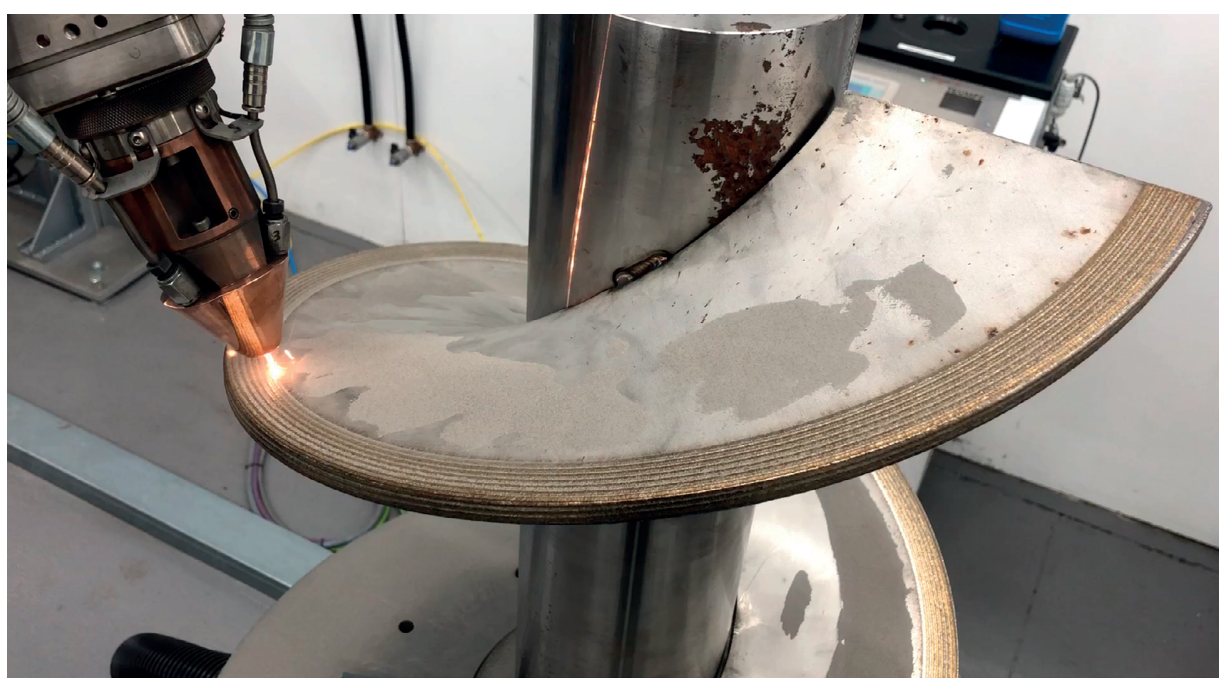

Additional degrees of freedom bring flexibility and complexity. For efficient process planning, a solid foundation of different algorithms must be developed. These must be capable of coping with major issues, such as the limited speed and reliability of AM technologies and the limited quality and aesthetics of the resulting products, all of which severely undermine the trust of technology users and adopters (Ford \& Despeisse, 2016):

- The parallel deposition is not usually capable of building overhanging regions without a support structure, since this will result in printing in the air. The exception to this limitation is using unbound material from preceding layers for support (like SLS of plastics or binder jetting). The unbound material acts like supports and need to be removed after the process.

- The surface quality heavily depends on the local surface curvature and normal orientation. It is possible to calculate predicted values of surface roughness and this will be used in Chapter 3 to compare the impact of traditional 2.5D AM versus multi axis AM.

Multi-axis AM algorithms require extra preparation efforts and process knowledge. In order to be competitive with the state-of-the-art three-axis AM process, the process planning for multi-axis AM must be automated as much as possible. Nowadays, the traditional computer-aided manufacturing (CAM) algorithms for material removal in subtractive manufacturing processes are already reaching a high level of automation. In some areas, such as the five-axis milling of dental implants, parts can be produced with a single-button solution. The end-user may not see toolpath being generated and may not be aware of any of the settings in the process. Even five-axis milling operations are currently programmed for minimal involvement by 
the programmer. Modern software only needs input for the necessary geometries and toolpath parameters. The rest is performed completely automatically. The toolpath is calculated, the spindle orientation is automatically oriented to avoid collisions, and the machining verification is carried out in an automated manner.

At first glance, the development of automated multi-axis AM strategies based on the experience of subtractive toolpath algorithms, e.g., milling, seems to be straightforward. However, although both algorithms use the same geometrical principles, they are substantially different.

- A milling tool may move along one line many times, but the result will be the same. However, if an AM nozzle deposits material several times in one place, the result will be difficult to predict because the material amount will increase every time. Therefore, AM planning must ensure no deposition of excess material. Besides the deposition volume aspect, the thermal aspect is also very relevant, where heating a certain spot several times or slower movement of heat source will cause more heat to be deployed and will change material properties. Where subtractive path planning algorithms do not take this into account, AM based path planning algorithms should.

- Additionally, the collision conditions change constantly. In five-axis milling, collision obstacles are defined beforehand and kept as granted. This makes collision check and collision avoidance straightforward and computationally robust. In the AM world, the possible collision objects change (grow) during the process, and the collision situation evolves continuously during the additive process. This is neither good nor bad. A sophisticated process planning approach may, perhaps, consider an order to build pieces of a part while avoiding collisions with the already deposited material.

In summary, most AM processes utilize three-axis kinematics and existing process planning concepts for these processes do not support multi axis kinematics. If part and/or nozzle reorientation during deposition will be supported via multi axis kinematics, then a new variant of process planning for AM is needed. The following section will define the research question based on this insight and describe the research methodology used to prove the outcome of this work. 


\subsection{RESEARCH QUESTIONS AND METHODOLOGY}

Multi-axis AM does exist, but it is non-optimal as reviewed in Chapter 2. Presumably, novel strategies may be elaborated as algorithms suitable for automated process planning to overcome major difficulties. Based on the analysis in the previous section, the following research question should be answered to enable reliable multi-axis AM capable of producing higher quality parts:

- To what extent modifications of existing subtractive path planning strategies and related algorithms can be used for multi-axis AM?

- Can development of novel multi-axis AM toolpath planning strategies help to overcome some of the challenges in AM by improving quality of the surface and reduction of the use of support structures.

- Can novel toolpath strategies for DED be developed that result in smooth deposition along 3D curves to improve surface quality?

- Can novel multi-axis AM toolpath planning strategies be developed that result in reduced or no support structure?

- Can novel multi-axis AM toolpath planning strategies be developed that result in improved surface quality by minimization of the stair-stepping effect?

The research approach for this work consists of a creative and experimental phase. The creative phase consists of a literature review and application development.

\section{Literature review}

The aim of the creative phase is to think and explore novel methods to address the research questions. Academic publications and commercially available products are reviewed to identify the state of the art in this technology and to propose potential solutions for further research in the field. The literature review focused on specific topics, such as the decomposition of solid models for multi-axis AM and multi-axis path planning algorithms for AM.

\section{Algorithm development}

Essential to the creative phase is the author's more than 20 years of research and development experience in the area of multi-axis milling (subtractive manufacturing) and path planning algorithms. This research and development effort has resulted in many commercially available products that define the state of the art in this field (ModuleWorks serves the worldwide CAD/CAM market for five-axis milling with around 70 percent global market share based on the number of CAM systems utilizing the algorithms).

The existing algorithms in multi-axis path planning for milling are reviewed to assess whether they are applicable for multi-axis AM after applying some changes and adaptations. For this reason, in this work, the comparison between path planning approaches for multi-axis subtractive and additive technologies is addressed. 
In this work, two new algorithms for multi-axis AM planning are developed to address the research questions:

- volumetric decomposition and 3+2 axis AM toolpath generation

- addressed challenge - minimization and reduction of support structures

- addressed challenge - minimization of stair-stepping effect

- toolpath generation for continuous five-axis AM

- addressed challenge - improvement of surface quality

- addressed question - to what extent existing milling path planning algorithms can be used to develop continuous five-axis AM

The developed algorithms presented in this work provide details about the geometrical and numerical aspects of the algorithms. The robust and implementable attributes of the algorithms are very important, especially since AM is expected to deal with unpredictable geometric shapes in the future. As such, the presented algorithms should not be limited to any particular family of geometric shapes.

\section{Experiments and validation}

Finally, the developed algorithms are validated either using computer simulation $(3+2$ axis decomposition) or machining tests ( $3+2$ axis FDM and five-axis DED). Measurement involves quantitative evaluation, while qualitative evaluation can also be performed based on an expert's opinion following a visual inspection. The combination of quantitative and qualitative approaches was necessary for benchmarking the experiment's yields because it allows grasping different kind of data that cannot be explicitly defined.

In the next chapter, a state-of-the-art review is performed, which is the basis for the creative phase of the above-described research methodology. The topics presented in this section are $\mathrm{AM}$, path planning for subtractive manufacturing, path planning for parallel 2.5D and FDM, path planning for multi-axis DED and known experiments from the existing literature. 

CHAPTER 2

\section{State of the art on part decomposition and path planning for multi-axis decomposition}


In the 1980 s and 1990s, it was believed that AM would replace subtractive machining. The new fabrication concepts promised easy production of very complex parts without the complications that usually accompany conventional processes. However, despite its simplicity, the use of AM technologies brings many issues. Problems are encountered at every step of the AM process, including data preparation, actual fabrication, making a proper part design for AM, finding a proper part build direction to reduce surface overhanging, slicing and hatching the geometry with eligible toolpaths, and setting the correct process parameters. Manufacturing of shapes with unlimited geometrical complexity with appropriate quality was expected to become trivial, but that has not been the case. To tackle multiple issues, companies and researchers have developed a variety of process planning algorithms.

This chapter addresses existing toolpath planning algorithms for multi-axis AM. The literature review of the relevant existing toolpath planning approaches will help to identify the similarities and differences between additive methods. This will provide an understanding of the drawbacks and gaps (flexibility, etc.) in the existing approaches so they can be addressed in the research. Furthermore, it will demonstrate how the AM community could benefit from filling these gaps.

\subsection{PATH PLANNING FOR MULTI-AXIS AM, KNOWN EXPERIMENTS FROM THE LITERATURE}

Although it is obvious that multi-axis AM could overcome some of the various limitations and inefficiencies of the layer-by-layer approach, multi-axis methods remain underdeveloped and widely unavailable for industrial usage. AM can be executed in many ways by applying different technologies. For instance, it includes the selective laser melting of metal powder, fused deposition modeling by extruding molten plastic filament, polymerization of selected volumes of liquid resin, additive welding technologies, etc. Every kind of additive technology has its own peculiarities that can create benefits or drawbacks in a particular application as described in Chapter 1. Despite being around for more than 30 years, most scientific publications deal with the traditional layer-by-layer $2.5 \mathrm{D}$ deposition process. To do multi-axis $\mathrm{AM}$, the process for toolpath generation is typically split up into two steps: model decomposition into sections (Section 2.1.1) and the actual strategies for path planning within those sections (Section 2.1.2).

\subsubsection{Basic decomposition strategies}

The current path planning algorithms are able to deal with highly complex products because they stick to the layer by layer approach. But this layer by layer approach treats the whole products the same, while some sections could benefit from other orientations of the nozzle and/or the product. Probably for each segment along a toolpath an ideal angle could be defined. This would result in very complex path planning algorithms. The solution used in literature is to decompose these more complex shapes into smaller, simpler volumes that can be easily processed by path planning algorithms, as shown in Figure 25. Several research papers highlight the importance of the decomposition of the part geometry as the starting point for 
multi-axis AM. As a result of decomposition, the part geometry can be represented as a set of several geometric features or primitives that can be processed using different slicing directions for each feature. Further the processing sequence of the features has great importance since some features require other features to be built, such they can be added on top of them, while potential collisions with already deposited material has an impact on sequencing strategy as well. Decomposition may be especially beneficial if there are strategies in place to slice all feature types in the decomposed object. The following paragraphs will discuss different decomposition strategies from literature, as well as their benefits and drawbacks.

Figure 25: An example of the volumetric decomposition of kitten figure (Wang, Zhang, Wang, \& Wu, 2014)
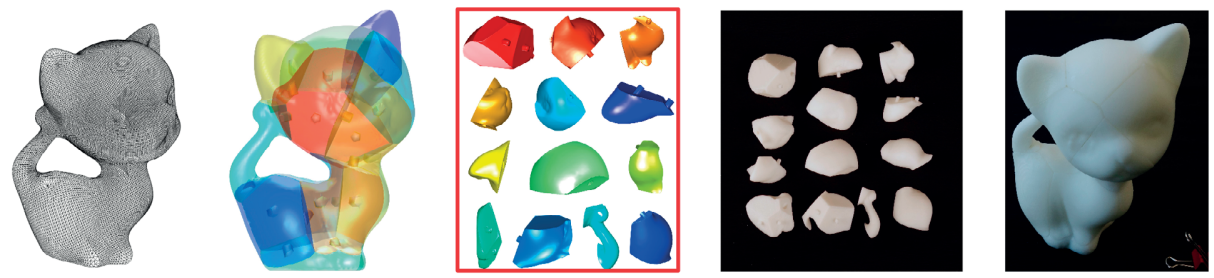

\subsubsection{Geometry-based decomposition}

Some decomposition strategies consider different geometric properties of solid bodies. For instance, decomposition may employ feature detection and extraction , , . This approach is suitable for parts that are created in CAD software with simple primitives, like extrusions, holes, cylinders, etc. Every feature will be assigned a best direction for being built. Based on how the geometric features are connected to each other and the preferred direction of the build, the slicing software assigns the best slicing direction. Some CAD parts include design history, which includes the creation of individual features so this information can be used to extract these features geometrically for decomposition. See Figure 26, for example, which shows orthogonal slicing of the extrusion directions of the features. Both models possess cylindric, conical and prismatic features. The revolved geometries are to be sliced orthogonal to the revolution axis, while prismatic parts are sliced orthogonal to the extrusion direction. This is the rule of thumb for such geometries. Any other direction will result in a deteriorated surface quality on non-flat areas. The goal of decomposing the part based on CAD design history is that relies on the heuristic that each feature will be sliced in its own directions. The drawback is depicted in Figure 26b, where the solid modeling extrusion direction of the features is used, which creates non-optimal results for the slanted surface (stair on the left slope of the top block). 
Figure 26: Feature-based slicing in different directions

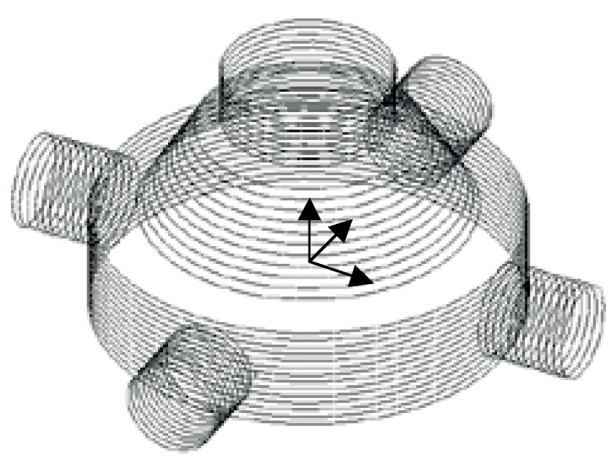

a) feature-based slicing (Sundaram \& Choi, 2001)

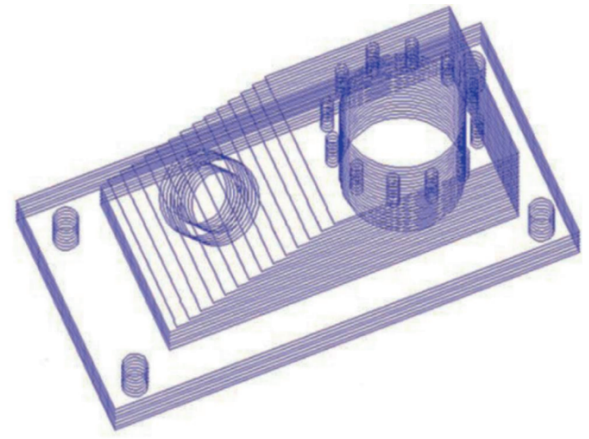

b) feature-based slicing ((Ding, et al., Automatic multi-direction slicing algorithms for wire based additive manufacturing, 2016)

Because of the limitations of the feature-based approach-in which organic shapes cannot be processed-Ruan suggests another approach based on the analysis of the feature geometry without prescribing a fixed direction for the best build direction. This approach relies on innovative geometry reasoning and analysis of the so-called tool-centroidal axis. The centroidal axis is computed as a line through the centroids of cross-sections. It contains geometry and topological information but is significantly computationally cheaper due to the fact that the centroidal axis is well defined in simple geometric shapes. Using a centroidal axis as a guide, the multi-axis slicing procedure can generate a 3D layer or automatically change the slicing direction as needed to build the part with a better surface quality. Figure 27 shows two connected geometric primitives. Each primitive has a centroidal axis (pink) that extends across the entire feature, meaning that the feature must be analyzed completely and fabricated at once. The proposed method suggests subdividing the centroidal axes into pieces to analyze whether this is possible, which improves the process planning if the feature is partially fabricated. The drawback is that this approach cannot be efficiently used for free form shapes because sometimes the individual cross-sections cannot be accurately identified.

Figure 27: Topology linking the centroidal axes of two separate part features (Ruan, et al., 2006)

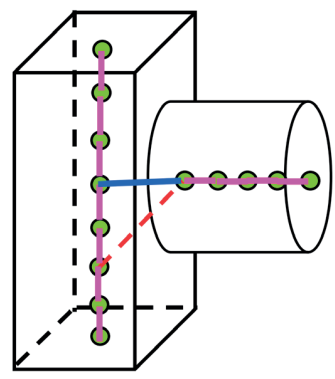


Besides analyzing the centroidal axis, some researchers reported methods based on a sweeping plane. Basically, a plane moves iteratively along the build direction and checks whether the next step violates specified conditions. Overhanging is used to detect whether or not a part's sub-volumes are feasible with the given build direction. One of the first papers on so-called multi-direction slicing for layered manufacturing was published in 2001 . Figure 28 depicts how the method works. For the part and build direction given in Figure 28a, the sweeping plane ascends from the bottom to the top. The part boundary (walls) is checked to ascertain whether an overhang occurs. If an overhang is detected (segment AB in Figure 28b), the detected faces and attributed sub-volume are marked as unbuildable and must be sliced with another build direction.

Similarly, applied feature based decomposition manually and validated through an experiment the elimination of support and reduction of stair-stepping effect, as Figure 29 demonstrates a part produced in two mutually orthogonal build directions.

Figure 28: Sweeping the unbuildable faces (Singh \& Dutta, Multi-Direction Slicing for Layered Manufacturing, 2001)

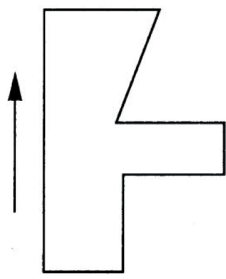

a) The model with the given direction of build
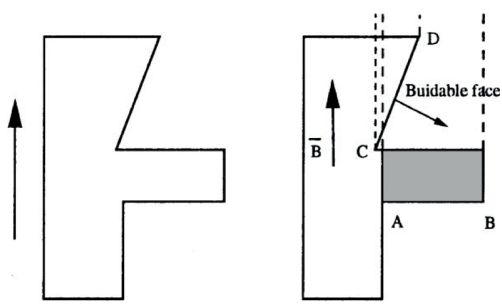

b) Sweeping the unbuildable faces

Figure 29: A handle fabricated with two build directions (shown with the green arrows) (Wulle, et al., 2017)

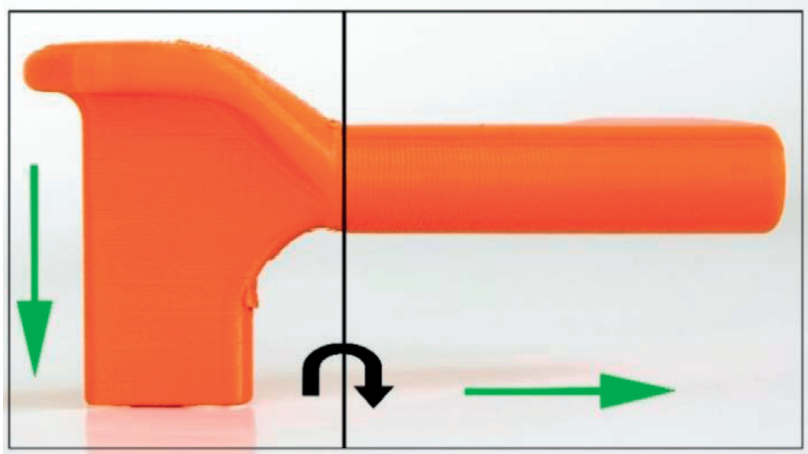


To perform a manual volume decomposition, the CAD user is splitting the part into pieces. Once the position and orientation of the part on the printing device have been decided, the user can make reasonable choices about how to split the part based on their experience, then define the order of printing and apply a normal layer based AM deposition strategy for each piece. This is the manual approach. The benefit is flexibility and an optimal outcome, whereas the drawback is that it requires a very skilled user and significant effort for each part.

A further common drawback of using geometric features for decomposition is that the reachability of the in-process model by the nozzle cannot be considered at all. While the decomposition of the part is independent of its orientation on the printing table, the physical process must take into account the printing hardware's ability to reach the positions so as not to collide with or generate overhangs. Therefore, geometric decomposition should consider the actual AM process in order to realize the benefits of multi-axis AM.

\subsubsection{Decomposition based on reachability}

The reachability-based decompositions in Figure 30 and Figure 31 show that the aim is to ensure that all areas can be reached and the printing head (nozzle) does not collide with the already printed material during the whole process. In Figure 31, this is achieved by having fixed orientations. In Figure 30, different colors show the growth stages of the part. Starting from the bottom, the volume expands so that its periphery is always convex. The drawback in the case of Figure 31 is that it is limited to a few fixed directions, which makes this approach unusable for arbitrary geometries. It also shows a relatively simple shape and very small decomposition elements where too many decomposition steps are performed due to focusing only on the convex hull and not respecting the geometric properties at all.

Figure 30: Convex-front peeling decomposition (Chengkai Dai \& Lui, 2018), (a) input geometry, (b) volumetric decomposition - color codes define reachability conditions
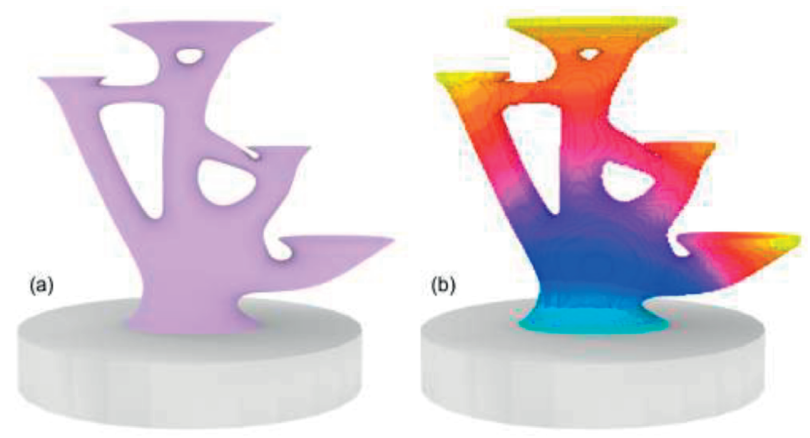
The following paragraphs describe a number of different publications and their approaches to multi-axis AM, where combinations of decomposition and reachability based approaches are presented:

The idea of mutually orthogonal slicing directions is presented where a part is built from six directions around a cube. Figure 31 shows how this approach works. The RevoMaker technique enables multi-directional printing via a rotational cuboidal base. The outer faces of the cuboidal base carry the partitioned geometries of the models, which are indicated in different colors. After printing on one face, the cuboidal base rotates so another face can be accessed by the nozzle. Temporary handles may also be deposited if they are required for re-clamping. Afterward, these handles can be mechanically removed. By defining fix directions and their sequence, the geometry is decomposed along these directions and reachability is respected at this stage such the collision of nozzle with the in process printed part geometry can be avoided. The drawback is the limitation to utilize only pre-defined fix directions independent of the geometric shape such this approach cannot be applied for arbitrary shapes.

- Figure 31: The RevoMaker build sequence (Gao, Zhang, Nazzetta, Ramani, \& Cipra, 2015)

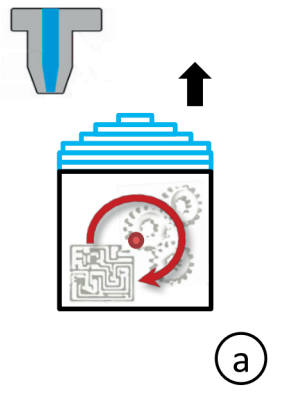

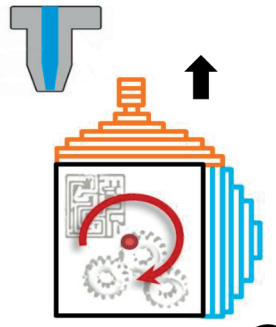

(b)

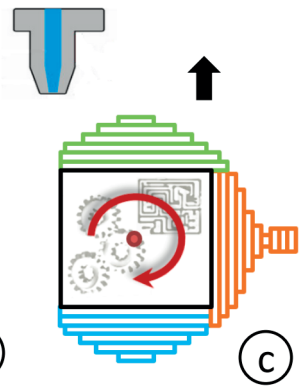

(c)

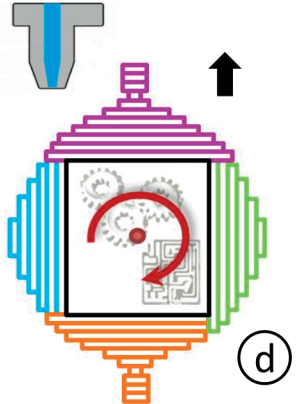

Decomposition method to utilize sweeping planes along the part shape helps to avoid reachability issues and collisions as long as the part geometry has a favorable shape for this approach. As shown in Figure 32a, each sweeping plane is defined independently from other sweeping planes. Essentially, each sweeping plane creates its own sub-volume. In contrast to Zhang, Wang suggests that the build orientation be preserved for some span. As shown in Figure $32 \mathrm{~b}$, the reduced variability of the sweeping plane orientation and the build direction generates several chunks with slightly different changes in the build directions. 
Figure 32: Slicing with the variable directional sweeping plane approach

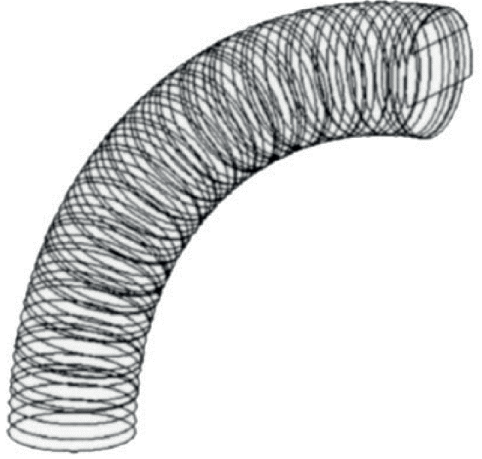

a) Variable directional slicing (Zhang \& Liou, 2004)

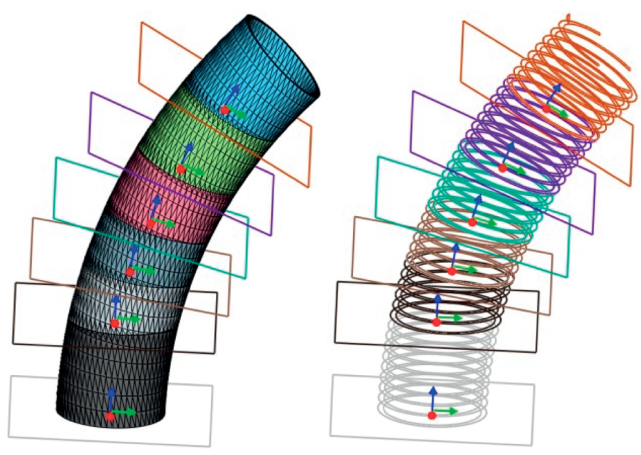

b) Adaptive slicing for the bend with variable segment thicknesses (Wang, Zhang, Wang, \& Wu, 2014)

\subsubsection{Overview and classification of multi-axis path planning for AM}

In contrast with the layer-by-layer approach, multi-axis AM is enriched with additional degrees of freedom that are assigned to the orientation of the additive end-effector (extruder nozzle, cladding torch or other devices used in the numerous variations of AM) or to the orientation of the part. There are two major aspects of how to define 3D spatial toolpaths: 3D points define a sequence of tool tip positions and the tool orientations at those positions. Additionally, tool orientations may change along the toolpath. Therefore, there are several possibilities for how to define multi-axis toolpaths, depending on tool orientation change and the tool tip trajectory shape. Some methods simultaneously vary the location and orientation of the nozzle, while others keep the nozzle orientation fixed for the entire process or subsections of the process. In the latter case, for each fixed direction of nozzle, classical 2.5D sliced tool paths can be applied, which can also be called "multi-axis" or "multi-directional" since the nozzle changes its orientation during the process. For the sake of clarity, the classification matrix of different AM toolpath strategies is suggested in Table 1 . The $3+2$ method implies that the nozzle orientation changes at some point, usually between geometric features. The 3D continuous method can also be executed on standard 3D printers with a fixed nozzle orientation, but in that case, the process cannot be considered multi-axis, since no additional rotational degrees of freedom are assigned to the nozzle. Therefore, a changeable nozzle orientation is a must for multi-axis AM.

Table 1: Classification of multi-axis AM toolpath planning approaches

\begin{tabular}{lll} 
& Planar toolpath & 3D curved toolpaths \\
\hline Constant orientation & $2.5 \mathrm{D}$ & 3D continuous \\
\hline Varying orientation & $3+2$ axis (multi-directional) & True multi-axis \\
\hline
\end{tabular}




\subsubsection{2.5D AM toolpaths}

2.5D toolpaths are just trivial flat toolpaths. The powder-bed-based processes are inevitably 2.5D additive processes, while the fabrication methods with different end-effectors, like torches or nozzles, must ensure that the end-effector orientation is preserved throughout the entire process. This is the most frequently used fabrication approach, and it can be seen in various types of AM technologies. The toolpath strategy typically considers contours and infill regions. The contour is often consisting of one or multiple, contour following toolpaths, while the interior has optimized toolpaths for speed of filling in FDM (Figure 33), speed of filling using laser with increased hatch spacing (PBF) or hatching patterns to reduce thermal warping (PBF).

Figure 33: Example of the most common infill patterns (Cain P., 2019)

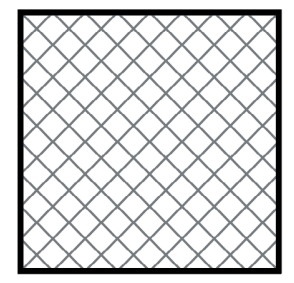

a) Rectangular

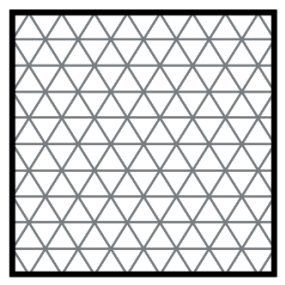

b) Triangular

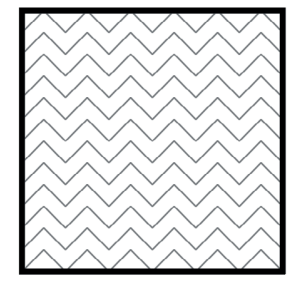

c) Wiggle

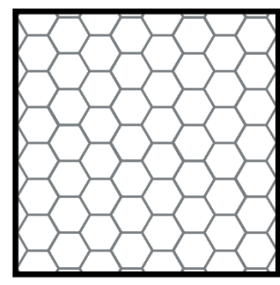

d) Honeycomb

\subsubsection{3D continuous AM toolpaths}

True multi-axis AM provides the greatest flexibility for efficient process planning. However, the complexity of the process planning for multi-axis AM differs drastically from other approaches. In order to cope with the increased complexity and the need to take into account all related issues, researchers and industrial adopters have decided to squeeze out at least some performance or quality gains while reducing some of the flexibility of the process. The way in which it has evolved is similar to the evolution of process planning for milling. Initially, milling was used for the machining of flat areas at different levels, which is very similar to layer-by-layer AM. Later, when the automation technologies reached a certain level, free-form shapes were machined using three-axis continuous milling, in which all machine axes (X, Y, and Z) could change simultaneously. A similar change occurred in AM. As shown in Figure 34, it was also suggested that the $Z$ coordinate be accentuated to track the nozzle along toolpaths on a curved surface. This advance seemed like a relatively straightforward and robust solution to remove the staircase effect on curved surfaces, but it also has some quality drawbacks. The thickness of 3D continuous layers is problematic as it cannot be kept sufficiently uniform on areas with different curvatures and slope inclinations, as shown in Figure 35. Basically, 3D continuous AM is distinct from 2.5D AM because of curved layers. Toolpath patterns, which were already discussed in Section 2.1.2.1, are generated or projected on the curved layers instead of on flat layers. 
Figure 34: Extruder path generation for 3d continuous FDM (Chakraborty, Aneesh Reddy, \& Roy Choudhury, 2008)

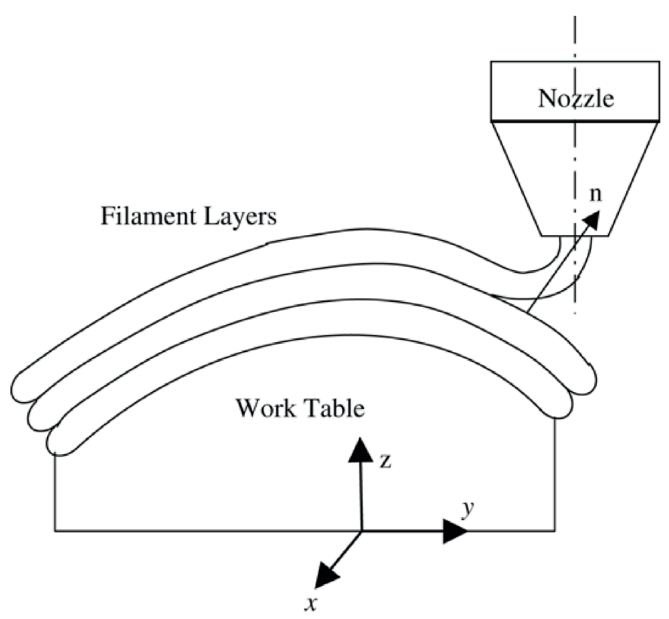

Figure 35: 3D Printing with clay (Interactive 3D Ceramic Printing: Sydney RobArch Workshop 2016) (Harvard Graduate School of Design, 2016)

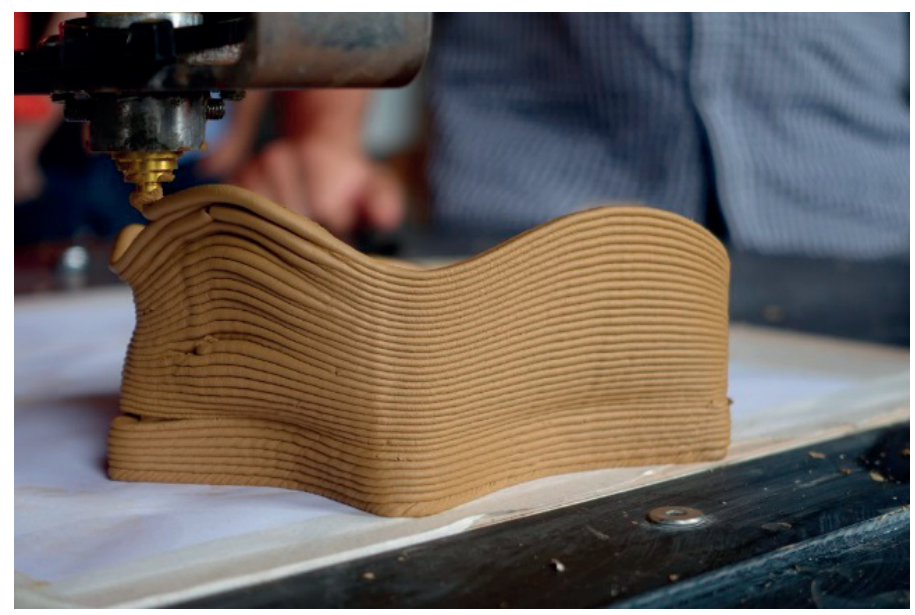

\subsubsection{3+2 (multi-directional) AM toolpaths}

The use of curved layers without aligning the nozzle orientation to the surface is not a widely used strategy. Most approaches tend to use either flat slices or a fixed orientation of the nozzle. Sometimes, both conditions are enabled, but the nozzle orientation is not kept constant throughout the entire build and changes for some slices. Some approaches use virtually no slices. Dwivedi, developed a path planning method to produce strut structures with a laser-based directed-energy deposition. This method allows the fabrication of uni-directional 
and branching slender structures. Figure 36 depicts an example of a tree-like structure. For every branch, the nozzle deposits material by moving co-axially with the branch growth direction. Basically, this method requires only the spatial coordinates of the nodes that define the beginning and the end of each line segment of the tree branches. This is a degenerate strategy with "empty" slices because virtually every slice (orthogonal to the tree branches) contains no toolpath pattern.

Figure 36: A branched structure with linear segments fabricated by a laser-based direct metal deposition (Dwivedi \& Kovacevic, A novel approach to fabricate uni-directional and branching slender structures using laser-based direct metal deposition, 2007)

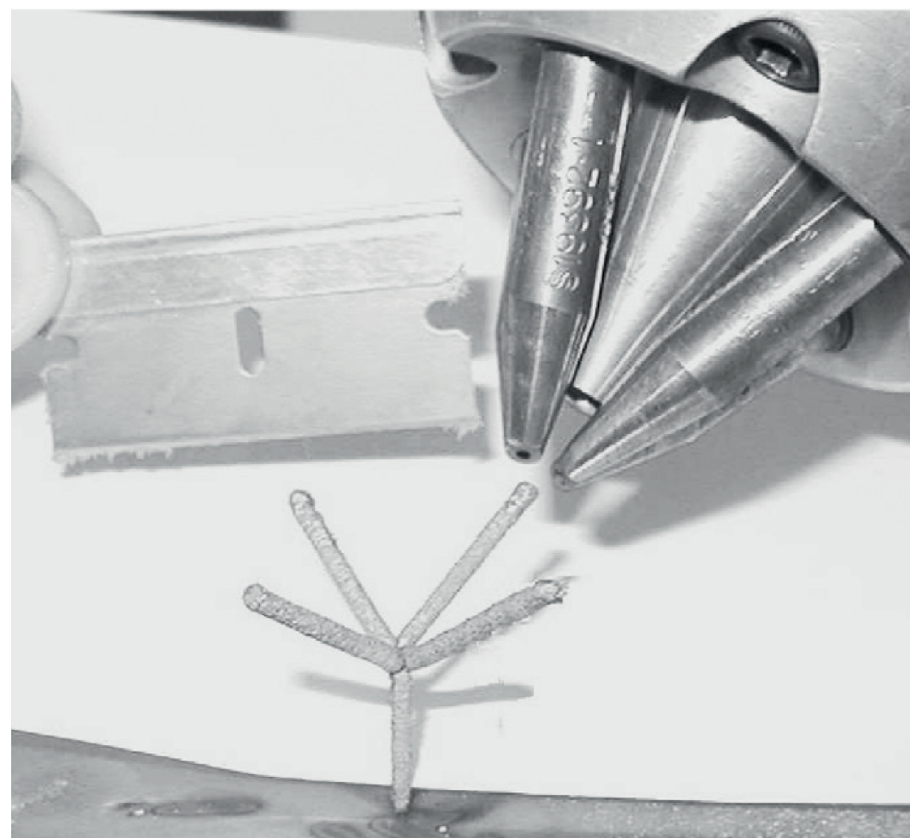

Another way to influence the process planning for flat slices is to variate the nozzle orientation. Kapil suggested using the standard slicing procedure combined with orienting the cladding torch tangent to the design surface, as shown in Figure 37. In addition, Campocasso extended this method to the fabrication of thin-walled structures. He limited the tool axis orientation in some areas, as depicted in Figure 38. Practically, the nozzle cannot be tangent at the cap due to collisions, so its orientation must be adjusted. 
Figure 37: Planar toolpath with varying orientation of cladding torch (Kapil, et al., 2017)

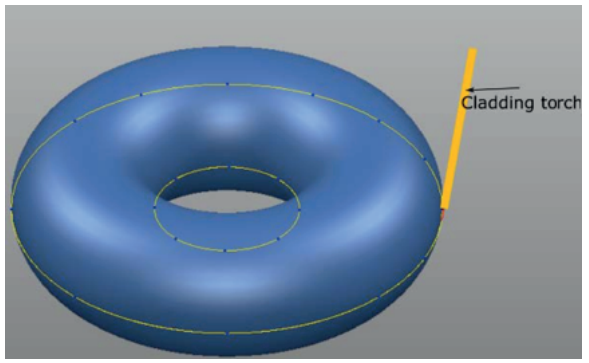

a) Cladding torch tangent to the object

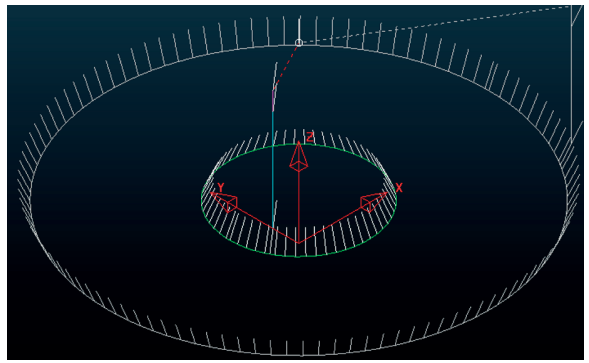

b) Five-axis torch orientations

Figure 38: 3+2 toolpath for thin-wall building (Campocasso, et al., 2018)

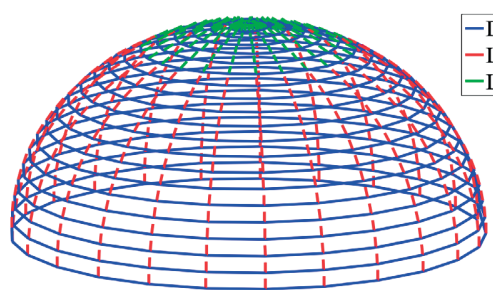

a) $3 D$ view

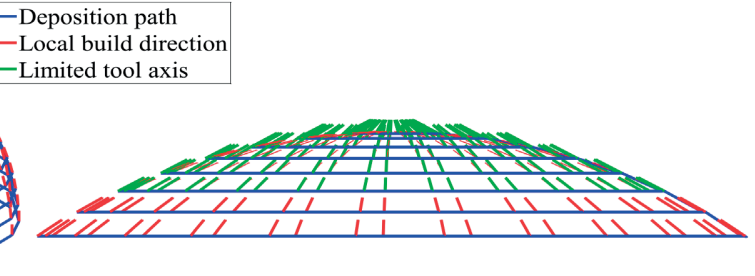

b) Zoom of the top (side view)

Selection of the proper nozzle orientation is a crucial task in multi-axis AM. Industrial trials showed that the smoothing of nozzle orientations along the toolpath and between adjacent layers may lead to a better surface quality since triangular, mesh-based geometry definitions have discretization artifacts. As shown in Figure 39, the corner regions or other regions with a large curvature (inverse of the curvature radius) are not numerically stable at slicing, especially if the triangulated mesh geometry is used as an input. Discrepancies in surface processing appear as abrupt changes on the tangent or normal vectors and therefore the orientation of the nozzle. A change in direction influences the thickness of the deposited material and it may leak into the gaps between the deposited layers. This situation must be considered in the post-processing step, when the nozzle orientations can be fixed. 
Gigure 39: Inconsistency in the nozzle orientations (Boisselier, Sankar, \& Engel, 2014)

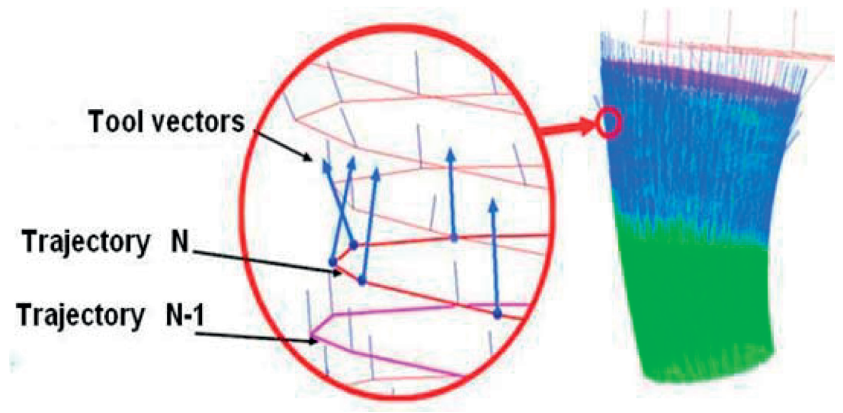

\subsubsection{True multi-axis AM toolpaths}

The oldest publication referring to the true multi-axis AM dates back to 1998 . Figure 40 shows an example of a part fabricated with early five-axis AM techniques. The toolpath is a spiral on a hemisphere where "the laser head (tool) orientation was compensated from the standard numerical machine tool orientation default of normal to surface, to normal to the edge for proper deposition."

- Figure 40: Directed light fabrication of a solid metal hemisphere using five-axis powder deposition (Milewski, et al., 1998)
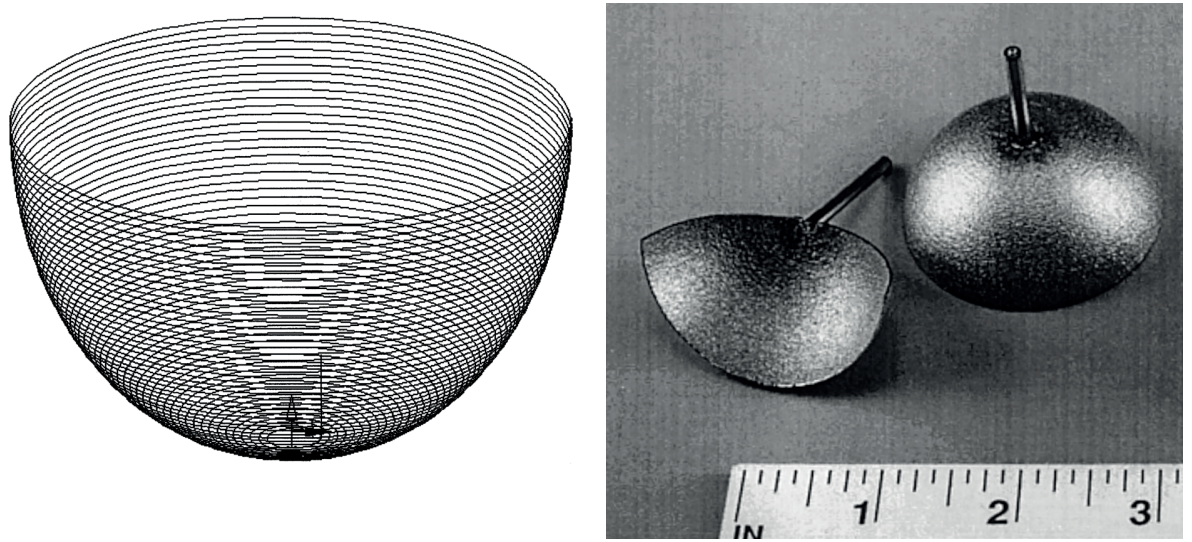

a) Spiral five-axis toolpath

b) Fabricated parts 
For very simple shapes, like hemispheres, the toolpaths and nozzle orientation can be formulated simply with an explicit mathematical formulation, just like in . Basically, in some cases, the use of dedicated software is not really needed. However, most of the parts in production are more complicated and have different geometric features, such as holes, shoulders, slots, or even free-form surfaces.

There are also layer-free technologies, and this approach was suggested in and . In this case, the AM process was implemented as resin solidification in a vessel, as shown in Figure 41 . In contrast to the uni-directional methods, this method allows varying of the "nozzle" orientation.

Figure 41: A schematic illustration of the CNC accumulation process (Chen, Zhou, \& Lao, 2013)

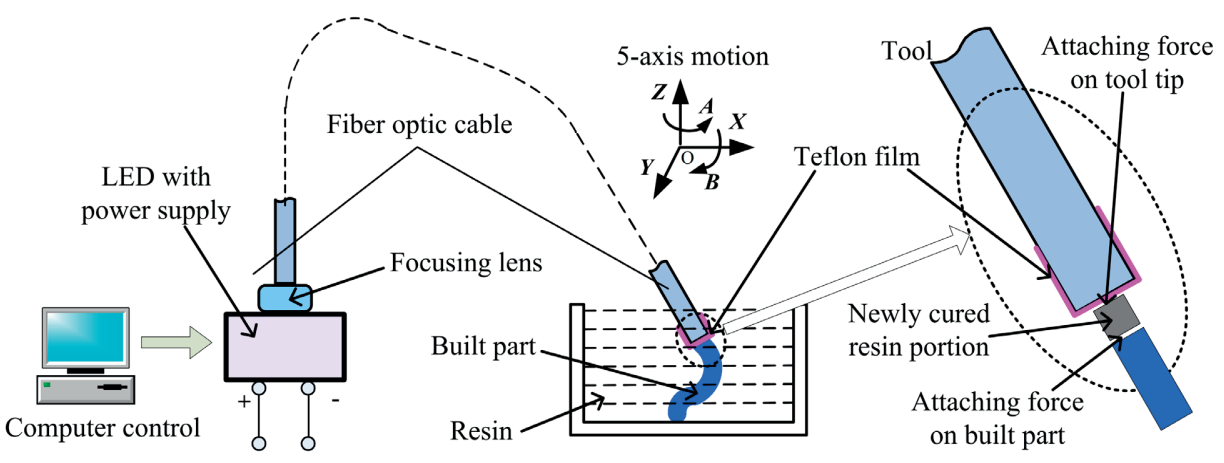

A true multi-axis application using thermosetting polymers and an ABB robot to move the nozzle has been presented by "Mataerial," a new form of AM that resulted from collaborative research between Petr Novikov and Saša Jokić from the Institute for Advanced Architecture of Catalonia and Joris Laarman Studio . Figure 42 shows how two components of the thermosetting polymer cause the material to solidify in the air, making it possible to print overhanging surfaces. 
Figure 42: 3D printing of instantly solidifying plastic (Chalcraft, 2013)

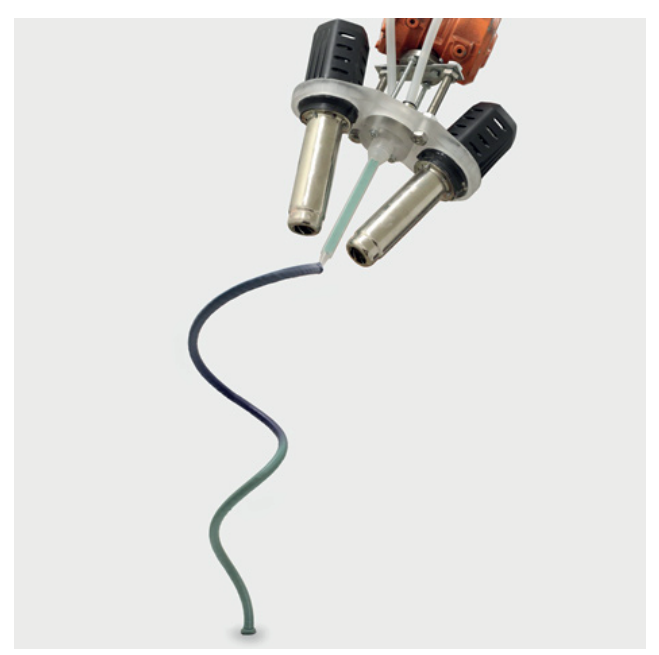

However, the more common approach is to define the orientation perpendicular to the outer surface of the product. As shown in Figure 43, the deposition tracks are placed on curved surfaces, while the nozzle orientation is always aligned with the surface normal. Unfortunately, this approach is unsuitable for non-convex geometries as well as situations where collision obstacles are present, which is almost unavoidable in real industrial settings.

Figure 43: Multi-axis nozzle orientations on a hemisphere (Campocasso, et al., 2018)

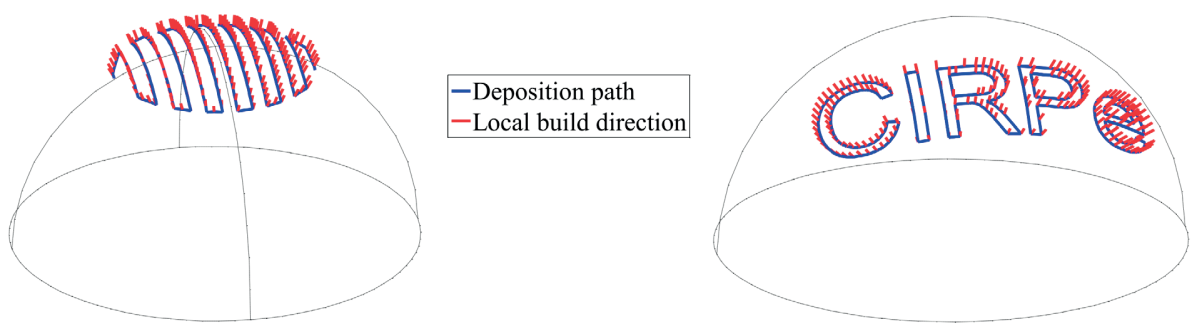

Some researchers have presented their findings on combining curved layers and enabling multi-axis nozzle orientation. Dwivedi built his method on feature-based slicing. Each feature is sliced with curved surfaces to generate curved layers, as shown in Figure 44. Then, within each curved layer, the orientation of the nozzle is set normal to the curved slice generator, as shown in Figure 44b. Additionally, layer scheduling was developed to prevent collisions between the nozzle and already deposited material. 
Figure 44: Slicing of impeller blades (Dwivedi \& Kovacevic, An expert system for generation of machine inputs for laser-based multi-directional metal deposition, 2006)

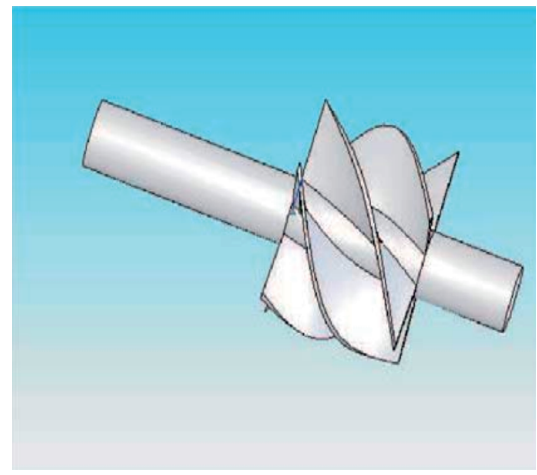

a) Impeller model

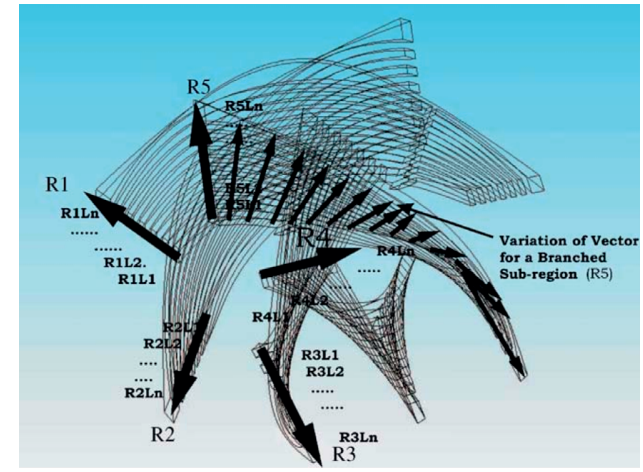

b) Slicing of blades

Similar to Dwivedi and Kovacevic, Kapil, Coupek, Chalvin and Singh all proposed a feature-based slicing approach that takes the parent feature, to which the sliced feature is connected, as the generator for the curved slice surfaces, as shown in Figure 45. The junction surface between the two features is repeatedly offset by the size of the layer thickness. The containment of the offset surface inside the sliced features is the curved slice.

Figure 45: Non-planar slicing of geometric features on the periphery of a cylinder (Kapil, et al., 2017)

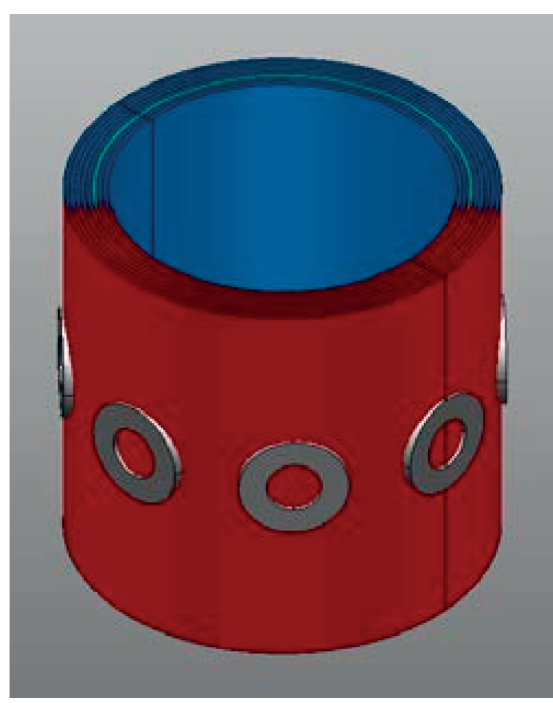

a) Ring feature on a cylinder

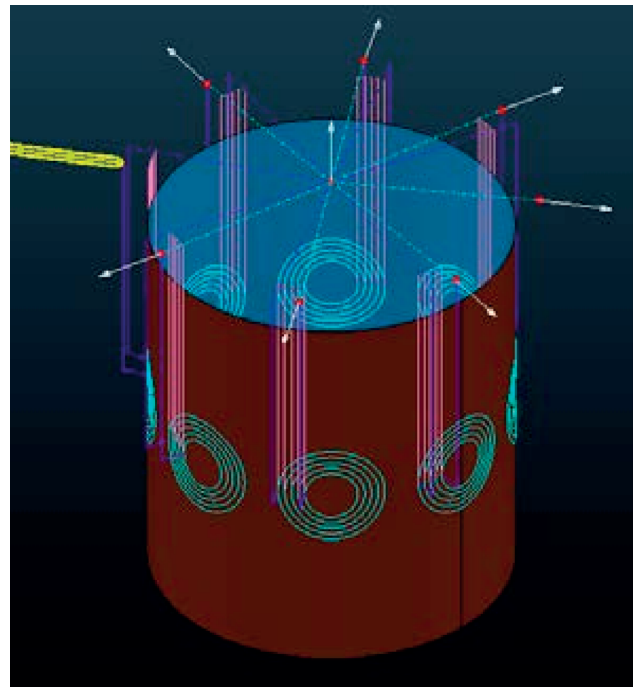

b) Cladding toolpaths 
Offsetting the floor surface geometry multiple times and intersecting with the feature to generate the curved slices might deliver towards the end slice geometries different from the initial ones such it will not necessarily result in a final sliced layer reaching the top surface shape fully. The result will then be multiple containments on the final sliced layers and thus disconnected toolpath clusters. This problem can be resolved by using intermediate layers that represent the morphing steps from the junction surface to the feature hull boundary, as shown in Figure 46. The algorithmic approach is to consider the volume to be produced as a cube topology parametrized by $u, v$ and $w$, where the user defines the four walls as well as one bottom and one top surface. A linear combination NURBS surface is interpolated using these six input geometries at each $w=$ const height, so this can be used as a variable thickness layer for multi-axis deposition. The drawback is the need for user input and the cube topology of the shapes.

- Figure 46: Intermediate curved layers (Isa \& Lazoglu, 2019)

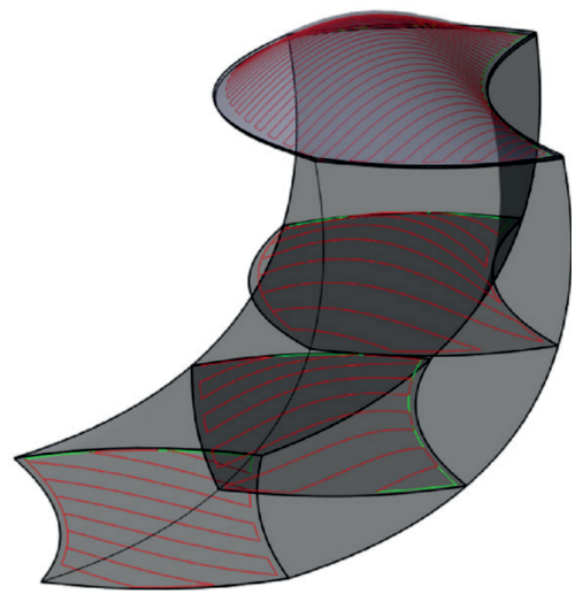

In addition to the described methods, there are several other methods that employ different derivation of the curved layers. The spherically curved layer $(\mathrm{SCL})$ model is designed to print overhangs. This method utilizes a continuous and synchronized interaction between the tool and the part by providing collision-free five-axis toolpath movements. The idea is that the nozzle is not allowed to enter the bounding sphere of the already deposited material, as shown in Figure 47. Another method also generates support-free and collision-free multi-axis toolpaths. Instead of plane slicing, this method uses a convex primitive in the form of a cube located on the table. At every iteration, it grows and changes its shape until it reaches the final desired shape. A convex hull after each iteration is calculated which guarantees collision-free decomposition of further cubes at the next iteration as illustrated in Figure 48. This approach 
generates many orientation changes during decomposition and does not focus on reducing the stair-stepping effect.

Figure 47: Spherical, curved layer (Jee \& Kim, 2017)

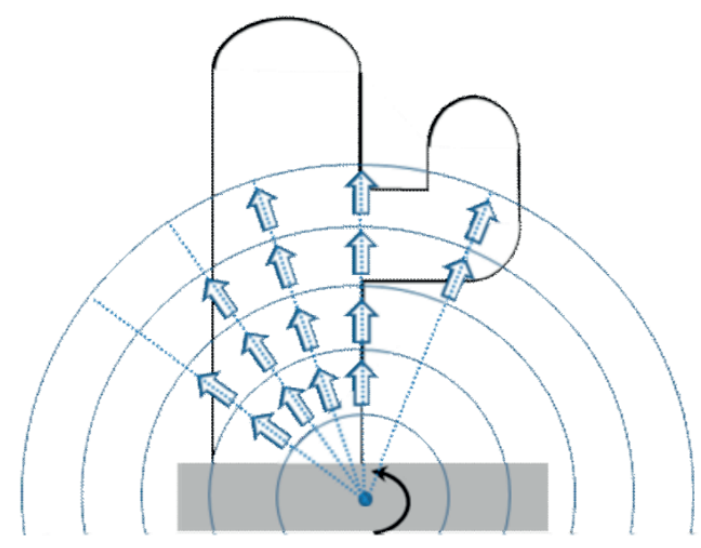

Figure 48: Convex-front peeling slices coded with the same color (Chengkai Dai \& Liu, 2018)

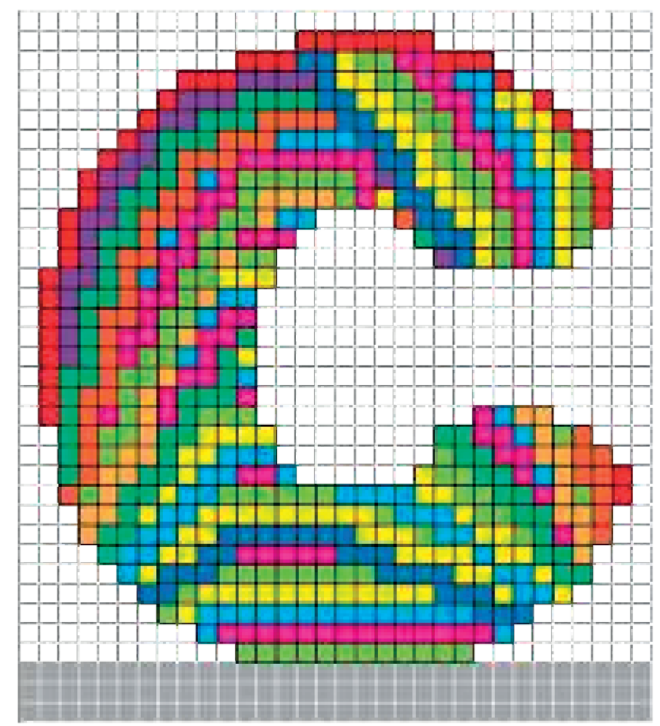




\subsection{CONCLUSION}

As described in the review of the state of the art in the previous sections, the following three demands of multi-axis AM could not be addressed fully: First, the approach must avoid manual operation of a trained user for geometry decomposition, such an algorithm must perform this step. Second, the approach must be able to work with arbitrary geometric shapes. Third, it must work with triangle mesh based data and should not rely on NURBS or other parametric surface definition as input for calculation. Same three demands apply to true multi-axis toolpath planning algorithms for AM. The automatic convex-front peeling approach from seems, so far, to be the most advanced and automatic algorithmic method to comply with the above listed three demands, but it does generate too frequent changes of nozzle direction which increases the machining time and reducing the surface quality. Further, it does not address the challenge of stair-stepping reduction, since the process is based on convex hull to eliminate collisions during printing and allow maximum reachability based on the frequently recomputed convex hull.

There are several gaps that can be identified between what the user wants and what is offered by the state of the art research:

- The existing 3+2 toolpath planning based on their respective decomposition algorithms aim to reduce or eliminate support structures and in some cases stair-stepping effects, but they are not providing a robust algorithmic approach which is independent of the geometry shape. Further they do not provide a direct optimization for these two goals. Accordingly, based on the shape of the part, these goals might or might not reached.

- The existing five-axis AM toolpath planning algorithms do not consider machine kinematics and potential collisions between part and the nozzle. Although collision avoidance is common in five-axis milling strategies, the demands on optimal nozzle orientation while being collision-free during AM is way more complex and demanding.

Closing these above gaps can improve the performance, quality and robustness of AM. For instance, a novel $3+2$ toolpath strategy should result in a better surface quality of the printed parts, whilst the production time can be reduced because of the elimination of the support structures. Improvements in the toolpath planning can result in smoother and safer nozzle movement.

The focus of this state-of-the-art review and the research presented in Chapter 3 and Chapter 4 is on the $3+2$ axis and true multi-axis AM from Table 1 with the aim to address the above listed gaps in current research and comply with the demands described.

In Chapter 3 a novel process planning strategy for $3+2$ axis AM is presented. This can be applied for $3+2$ axis FDM and DED. Transforming the results to further AM processes might be possible, but that topic will not be covered in this thesis. 
The novel $3+2$ axis AM will address the following points:

- It is able to work with part definition based on triangular mesh, such there is no requirement for the user to provide surface or solid model CAD part definition.

- The algorithm for path planning and decomposition does not require the user to manually define and select individual features, such there is no dependency of expert user

- Reduction of stair-stepping effect and elimination or reduction of support structures are directly addressed by the algorithm as primary optimization criteria

- Algorithm is designed to work with arbitrary part shapes

- Algorithm guarantees avoiding any collision between in-process part and nozzle

Chapter 4 presents a novel true five-axis AM process planning strategy, which will address the following points:

- It is able to work with part definition based on triangular mesh, such there is no requirement for the user to provide surface or solid model CAD part definition.

- The algorithm for path planning requires from the user only the selection of the area where the deposition should be applied and does not require creation of further auxiliary geometries

- Smooth nozzle orientation change is applied

- Algorithm is designed to work with arbitrary part shapes

- Algorithm guarantees avoiding any collision between in-process part and nozzle 

CHAPTER 3

\section{Development of novel process planning strategies for $3+2$ axis FDM and DED}


The two process planning strategies presented in this chapter aim to utilize the additional freedom of multi-axis hardware compared to three-axis hardware to address some of the existing and known limitations of three-axis FDM and DED, as described in Chapter 2 . As presented in Section 2.1.2, 3+2 axis AM is a subset of multi-axis AM and it locks the two axes (e.g., rotary axis depending on the hardware kinematics), then applies three-axis material deposition in a layer-by-layer process, just like in a three-axis FDM or DED process. After an orientation change, three-axis AM is applied. The novelty of the approaches described in this chapter is the creation of a process planning algorithm that applies a geometry decomposition of the given part model for $3+2$ axis AM based on the process-specific constraints and optimization goals, all of which can be defined by the process planner.

$3+2$ axis AM can become a game-changer for industrial AM applications. For instance, PEEK material is particularly attractive for mechanical rather than chemical approaches as it creates enhanced sterility in surgical tools and devices. PEEK can be used with the FDM method and the processed product inherently possesses all related drawbacks. However, the support removal and surface finish of FDM parts made from PEEK can be less than desirable because of PEEK's "bad" machinability and high resistance to most solvents.

Usually, process planning for multi-axis AM begins with model decomposition. Variations of feature-based decomposition have been presented by many researchers. The core principle is to distinguish between the geometric features so each of them can be fabricated using a fixed nozzle orientation, as shown in Figure 49.

Figure 49: $3+2$ slicing a part with two features

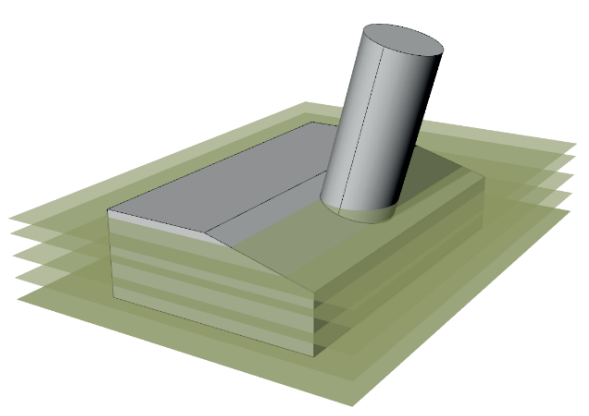

a) Slicing of the bottom block

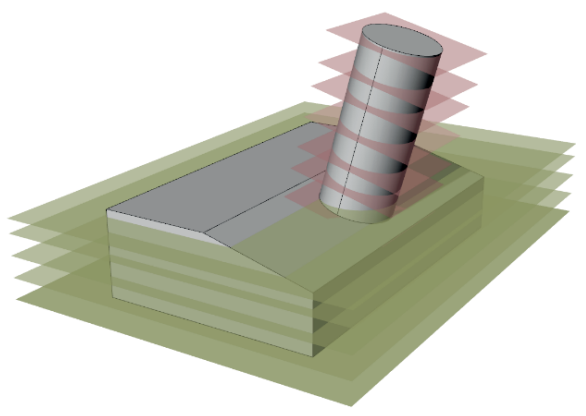

b) Slicing of the top cylinder

However, this approach presents at least two challenges. First, connecting geometries between geometric features is not as simple as it is for parent features. Thus, it may require alternative planning, as highlighted in red in Figure 50. Second, recent trends in design for AM predict widespread use of topology optimization, which is likely to result in free-form shapes with barely recognizable features. Several research papers have focused on decomposition of 
the part geometry as presented in Chapter 2. As the result of decomposition, the part geometry can be represented as a set of geometric features or primitives that will be processed using different slicing directions. Different decomposition strategies consider the different geometric properties of solid bodies, such as feature detection and extraction, as in and, the detection of the undercut surfaces, inscribing a cube inside the model, the morphological skeleton of the solid, as in and, and the topology and centroidal axis. In the state-of-the-art research on different decomposition methods for $3+2$ axis AM, all studies (except) apply decomposition algorithms to the given part shape without respecting the positioning and orientation of the part on the hardware (e.g., table) at the decomposition stage. In contrast, the algorithm that will be presented in this section respects the position and orientation of the given part on the table within the core algorithm where decomposition happens, so it can find the optimal solution for the same shape at different orientations and positions. Finding proper decomposition volumes and build directions improves the resulting surface quality.

Figure 50: Feature-based decomposition of T-shaped geometry
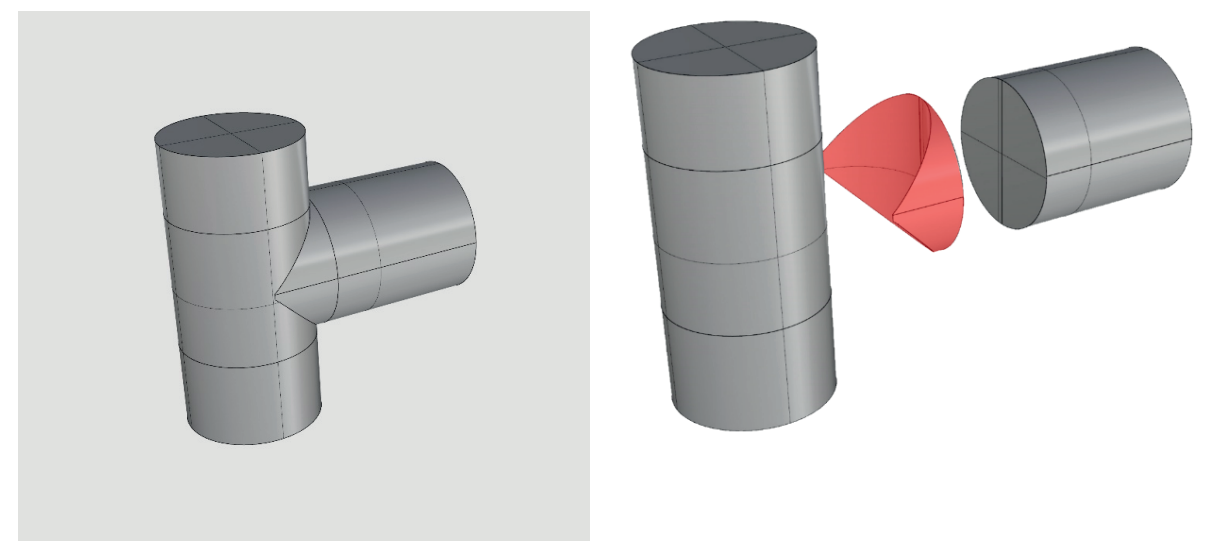

a) Two merged cylinders

b) Two decomposed cylinders and the connecting feature (red)

For each decomposed volume, a build direction must be assigned. The build direction defines the rules for how slicing will be carried out, i.e., deriving orientations of the layer planes. The orientation of the layer can either be fixed for all layers (parallel slicing) or may vary and depend on some specified criteria, usually as a function of the part or feature geometry. Build directions may be defined as functions of various metrics, such as surface accuracy, time to build, or number of slices, as suggested in. The sequencing of the build order in which the decomposed volumes must be fabricated is also very important. As parts of one solid body, the decomposed volumes are to be produced in an order that assures the physical existence of a base substrate on which to deposit the material (of either the same volume or an adjacent one) „.. Arranging the layers in a suitable fabrication order creates not only a material adja- 
cency problem but also brings up the issue of collision between the nozzle and the already deposited material. Several solutions have been proposed, ranging from rescheduling the order of subdivided sub-volumes to changing the nozzle orientation , ,, (Singh \& Dutta, 2003),

This chapter describes a geometry-based concept to decompose the product into discrete volumes by using space partitioning with infinite planes, all while considering the advantages and constraints of multi-axis additive manufacturing. The discrete volumes are used to generate process planning variants and to compute and generate boundary conditions for such process planning strategies. In addition, this planning can incorporate more complex scenarios, so overhangs, nozzle orientation and gravity can be considered. This development eliminates the gap between the existing algorithms, which require knowledge of the part design (properties of features included in the part geometry) or analysis of the centroidal axis. The algorithmic framework developed for the multi-directional planar decomposition of volumes for $3+2$ axis AM processes is robust, and it is based on analysis of the relationships between points, planes, and mesh surfaces. The method describes a computational geometry algorithm where a volume is split with a plane, with due consideration given to the specific constraints of additive manufacturing. No derivation of surface curvature or other properties is needed. This thesis focuses mostly on resolving the geometric problems that arise when identifying a plane position for volume decomposition for the sake of additive manufacturing process planning (upskin/downskin and other criteria may be taken into account). The algorithm generates $3+2$ axis slices that require no support structures.

In this chapter, two algorithms are presented. First a general algorithm using brute force optimization method is described and implemented. Second, an algorithm based on greedy method is presented, where computation speed is improved by further limiting and simplifying the search window for optimal solution 


\subsection{DEVELOPMENT OF AUTOMATIC 3+2 AXIS AM PROCESS PLANNING FOR NON-BRANCHING SHAPES}

Figure 51 shows a part geometry and how the layers are aligned in three-axis FDM to produce this part, as well as the support structures added by state-of-the-art three-axis FDM process planning to allow the overhang region of this geometry to be built.

Figure 51: Scheme of 3D printing with support structures

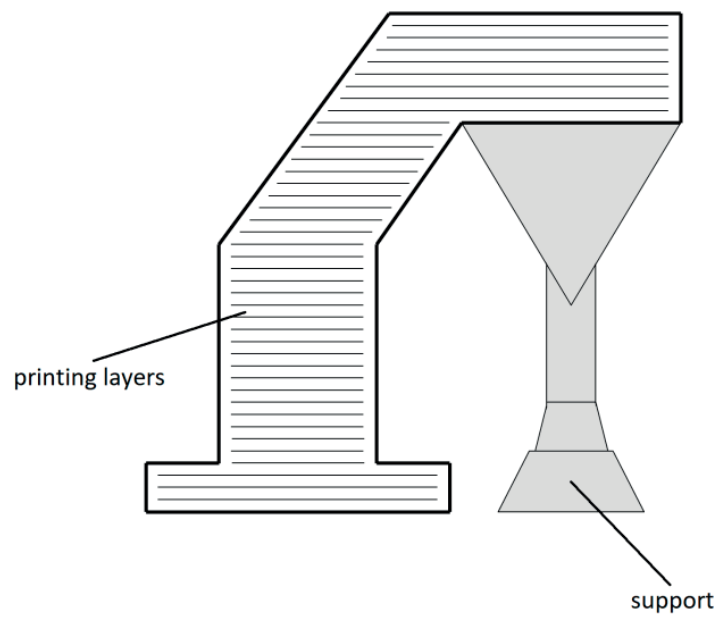

For various reasons, the elimination of supports for FDM and DED are beneficial. Creating supports increases the production time, the material used and, most importantly, adds the extra step of eliminating them at the end of the process. In FDM, these supports can be created with a different (e.g., salvable) material, but in DED, they are typically created using the same material as the part and require subtractive methods to remove them after the deposition. On hybrid machines like the DMG MORI hybrid CNC machine, this can be completed on the same machine. Usually, however, the part is removed from the AM machine and reclamped on a further machine to remove the supports. This adds an extra step and increases the cost of the overall production of the part.

Figure 52 shows how, in three different orientations (1), (2) and (3)), the same part shown in Figure 51 can be printed without supports. In addition to eliminating the need for supports, the stair-stepping effect caused by subsequent layers is significantly reduced in Figure 52 compared to Figure 51. This will be explained more in detail later in this chapter. 
Figure 52: The geometry from Figure 51 printed without support structures

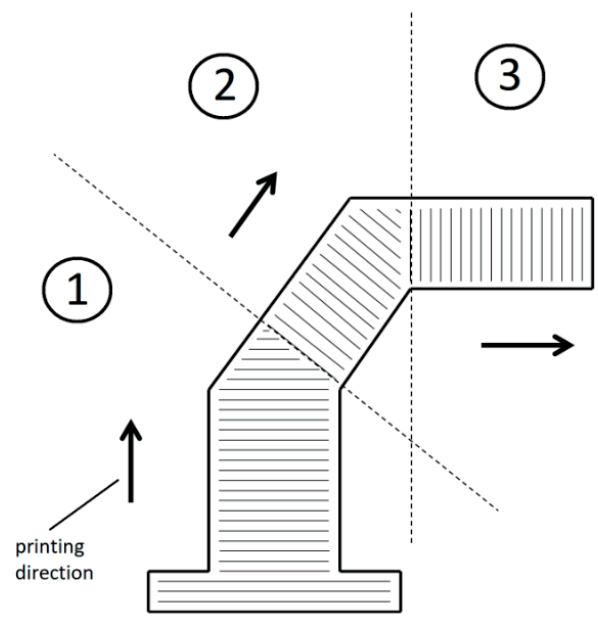

In order to tackle the general problem of manufacturing any part shape, the part must be reduced to geometries that comply with some geometric criteria. In this case, it is non-branching geometries. Two algorithms are presented below to solve the $3+2$ multi-axis AM process planning problem for such geometries. In the future, the geometrical constraints can be loosened so a wider range of parts can be produced using a more general algorithm. The presented algorithm is implemented on a personal computer and some geometries are presented to test the algorithm.

\subsubsection{Algorithmic problem definition}

The part shape shown in Figure 53a can be 3D printed using 3+2 axis FDM or DED hardware. There are no accessibility constraints throughout the deposition process and the nozzle is conservatively approximated by a half-space using its tip position and orientation. $3+2$ axis FDM should be able to eliminate to print support for this geometry which is needed in classical 3D printing. Further branching of the geometry is excluded for this part shape.

Figure 53b shows how this part will be printed using three-axis hardware, whereas Figure 54 shows how this part can be printed with a $3+2$ axis toolpath if the orientation is changed during the printing process. This requires the space to be split into two partitions, which will be addressed in the algorithmic description below. 
Figure 53: Slicing of a non-branching part geometry

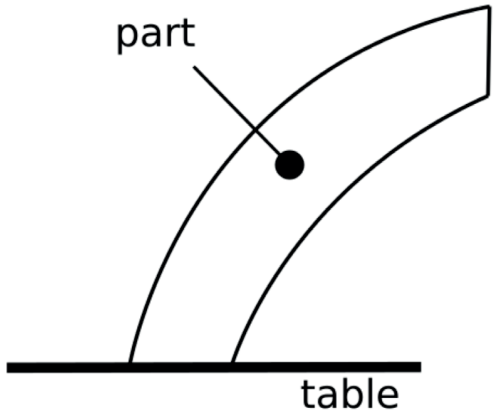

a) Problem definition

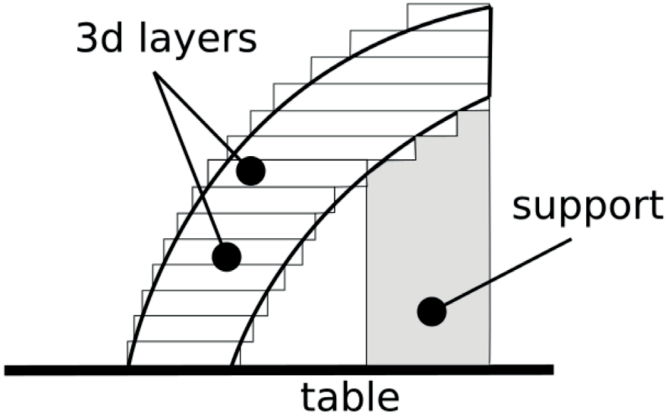

b) $3 D$ printing without multi-axis

In Figure 54, it is clear that using more axes would result in greater benefits. The problem is that no systematic method exists to generate toolpaths for products of random complexity. In this case, the complexity is defined as non-branching parts. The goal is to develop a method for automated subdivision of this subset of geometries. Basically, the algorithm must calculate the positions of the split planes that define the print directions for each subsequent sub-volume. To simplify this algorithmic approach further, let's assume that our part can be printed by using two subsequent 3D printing directions and we already know the first printing direction, which is $z$ direction. The problem is then reduced to:

1) Find the second printing direction;

2) Find the split plane in space where the given part is decomposed into two pieces.

Figure 54: $3+2$ slicing of a non-branching part geometry

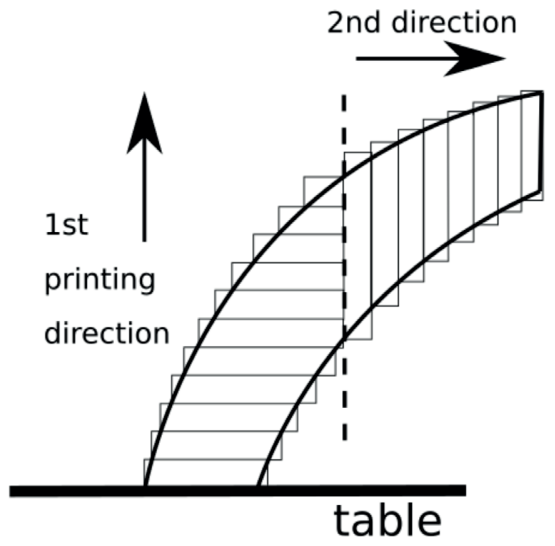


While analyzing the surface geometry in the vicinity of the intersection between the scanning plane and the part surface, the scanning plane may adjust its orientation to search for its optimal position. Figure 55a depicts an example of the vertical plane in which the black dashed line is the first optimal border between the two operations. Depending on the optimization criteria, other results may be selected, such as, for instance, in the case of the blue or red dashed lines shown in Figure 55b.

Figure 55: Alternative $3+2$ slicing

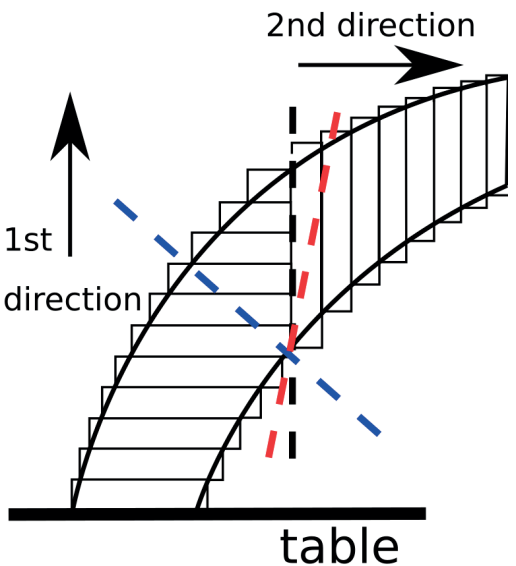

a) Layers for decomposition with a vertical plane

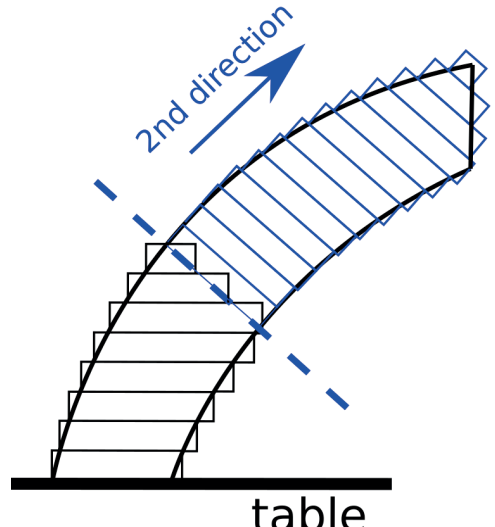

b) Layers of alternative decomposition

\subsubsection{Algorithmic premises for geometric analysis of meshes}

Given that the part is a triangular mesh (STL file) with $n$ triangles, each triangle has a facet normal that is oriented outward when the facet vertices are ordered counterclockwise and the object is observed from the outside. Note that the triangular mesh must build a closed solid geometry so that the material side and air side of each triangle is properly defined with the facet normal being shown from the material side to the air side.

The algorithm searches for a split plane $E$, as shown in Figure 56 . The split plane $E$ can be at an arbitrary position and have any orientation except for the first printing direction. Additionally, the split plane $E$ must satisfy certain criteria, which are described later in this chapter. The algorithm aims to calculate $\overrightarrow{\mathbf{r}}_{2}$ and $\boldsymbol{S}$, both together defining the split plane $E$ as seen in Figure 56. Then, each split sub-volume can be sliced according to its printing direction, as shown in Figure 57. 
Figure 56: Definition of the geometries required or used for computation

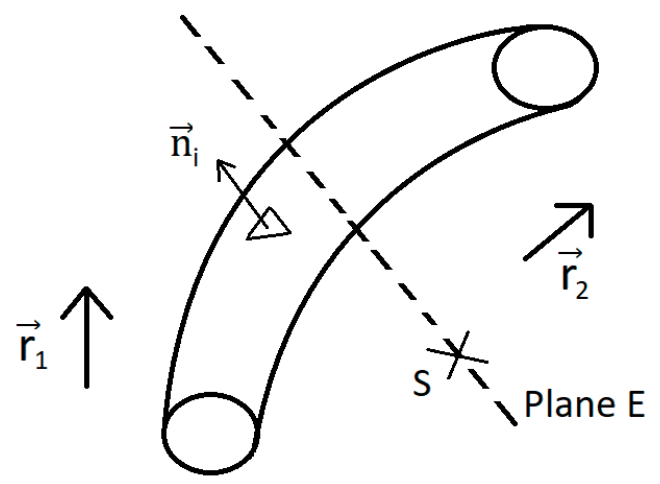

, where $\overrightarrow{\mathbf{n}}_{i}$ : normal vector of triangle $i$;

$\overrightarrow{\mathbf{r}}_{1}$ : first printing direction $(\mathbf{0}, \mathbf{0}, \mathbf{1})$;

$\overrightarrow{\mathbf{r}}_{2}$ : second printing direction;

$\boldsymbol{S}$ : point defining the split plane E (plane's normal vector is collinear $\overrightarrow{\mathbf{r}}_{2}$ ).

- Figure 57: Part splitting and slicing

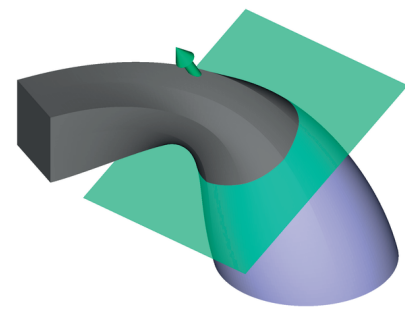

a) Part and split plane

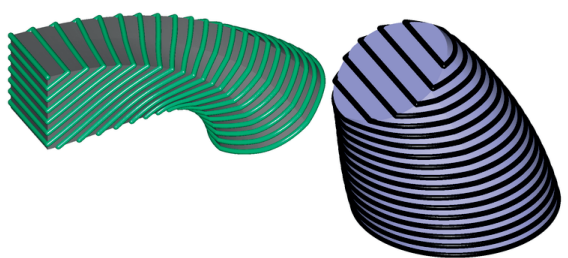

b) Two 3+2 sliced sub-volumes 
As presented in Figure 58, the area of a triangle $A_{i}$ is defined here as each triangle can have a different size. Later in the algorithm design, the influence of the area size of the triangle will be addressed.

Figure 58: Definition of a triangle

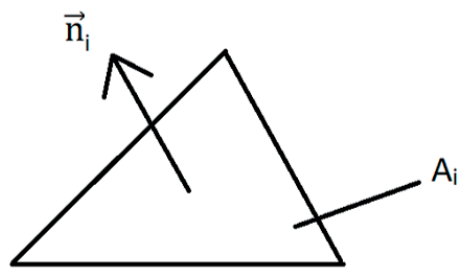

Figure 53b shows the staircase effect caused by the layered deposition. Each layer is an extruded 2D geometry (extruded along the printing direction) with a finite thickness for each layer. Subsequent layers are based on different base 2D geometries, which is the cause of the staircase effect on the surface of the printed part. If the 2D geometry does not change from one layer to the next, there will be no stair-case effect, so the surface roughness coming from stair-case effect will be zero. Figure 59 demonstrates this outcome. The 2D base geometry between that layers at a particular location does not change. Mathematically, this can be formulated as the triangle being orthogonal to the printing direction. A good printing process aims to reduce the surface roughness caused by this staircase effect. For example, see Figure 60 , where the triangle (visible as a line due to side view) is not orthogonal to the printing direction so the staircase effect is visible. On the other hand, as shown in Figure 61 , if $\overrightarrow{\mathbf{n}}_{i}$ is parallel to the printing direction, the staircase effect is minimized. The best printing results are achieved if the printing direction and triangle are orthogonal to each other. Deviation from the ideal angle of 90 degrees can be a significant characteristic for defining the error function. This scheme was considered by to predict the scallop height of the staircase effect. The scallop height is computed as the shortest distance from the layer tip or corner formed by adjacent material layers to the surface of the part model, as shown in Figure 62. Therefore, the scallop height is a cosine function of the angle due to the scalar product, such it is depending on the layer thickness and the surface slope, as following:

$$
\text { cusp height }=\text { layer thickness } \times\left(\vec{n}_{i} \cdot \vec{z}\right)
$$


Figure 59: Staircase effect when slicing upright surfaces

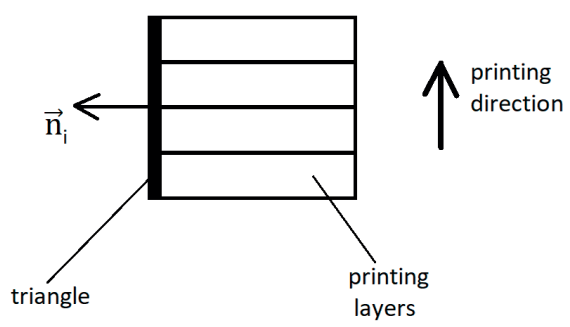

Figure 60: Staircase effect when slicing sloped surfaces

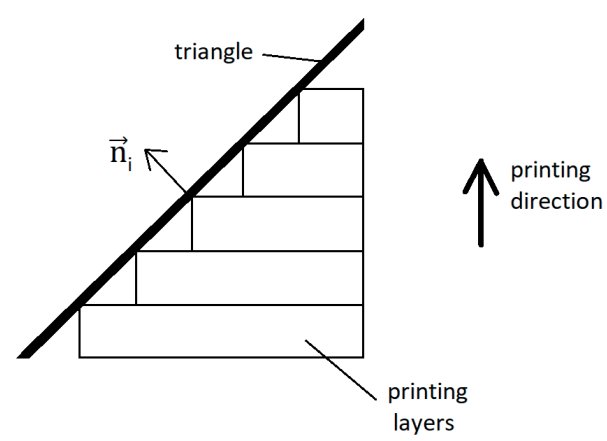

Figure 61: Staircase effect on flat surfaces

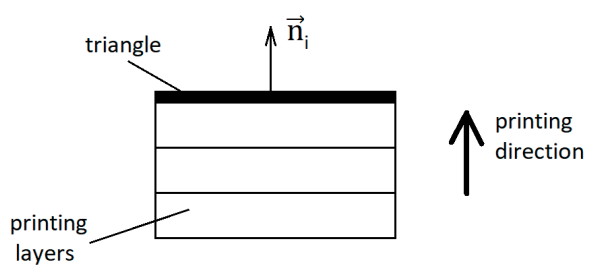

Figure 62: Staircase effect and cusp height calculation (Dolenc \& Mäkelä, 1994)

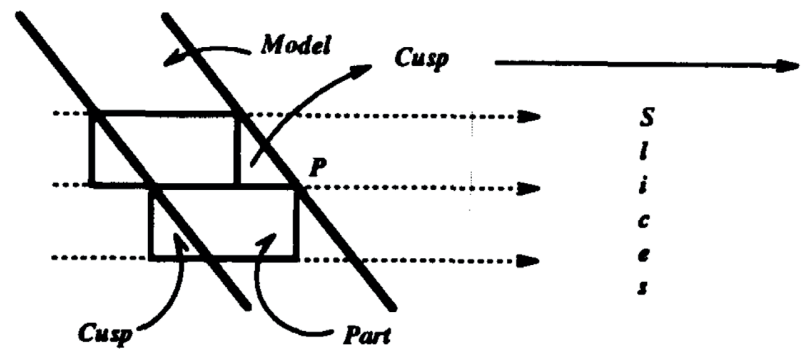

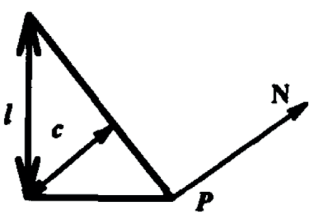

Cusp height 


\subsubsection{Half-space decomposition}

Since we have considered only one split plane at one iteration, the split plane defines two half-spaces located from the opposite sides of it. The split plane separates the sub-volumes of the part for process planning. One half-space includes the geometry to be fabricated with the first printing direction, while the other half-space includes the geometry to be fabricated with the second printing direction. The concept of half-spaces is also necessary to analyze mesh triangles for overhang and the staircase effect, as well as to identify an optimal position for the split plane.

Figure 63 defines the first and second half-spaces split by plane $E$ and the notation of triangles $T_{1, i}$ and $T_{2, i}$ residing in first and second half-spaces.

The half-spaces are defined as follows:

(1): half-space of the first printing direction

(2): half-space of the second printing direction

(1) and (2) are separated by $E$.

Figure 63: Definition of triangles after splitting of the space by plane $E$

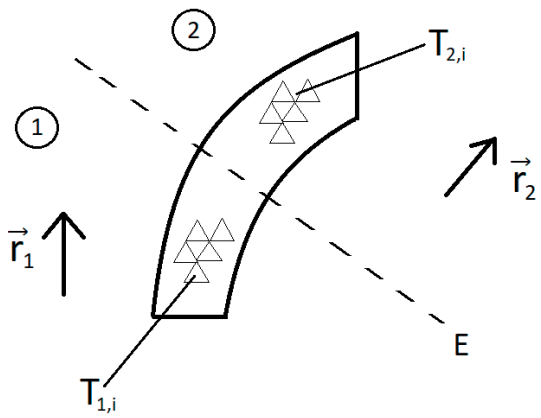

Let $T_{1, i}$ stand for all the triangles (vertices and normal direction) in a half-space (1) after separation from the 3D space by plane $E$. Accordingly, $T_{2, i}$ stands for triangles in half-space (2).

A plane in 3D space has only two degrees of freedom. As shown in Figure 64, plane orientation $\overrightarrow{\mathbf{r}}_{2}$ requires two rotational degrees (two Euler angles, pair $\varphi$ and $\theta$ ). These two Euler angles are used in the search function as independent variables, as shown in Equation (2). Besides the angles $\varphi$ and $\theta$, the distance $d$ of the plane to the coordinate system origin must be specified and be in the range [dmin, dmax], defining the minimal and maximal allowed distances. 
Figure 64: Plane definition with Euler angles

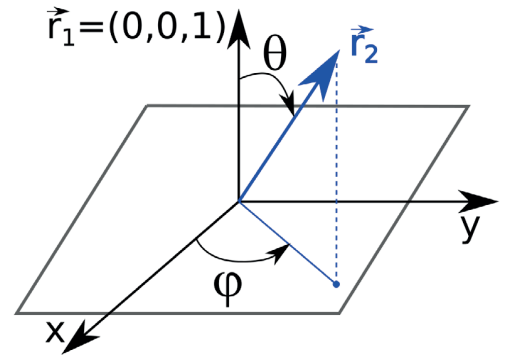

$$
\begin{gathered}
\left\{\begin{array}{c}
\varphi \in\left[0^{\circ}, 360^{\circ}\right] \\
\theta \in\left[0^{\circ}, 180^{\circ}\right] \\
d \in[d \min , d \max ]
\end{array}\right. \\
\overrightarrow{r_{2}}=\left\{\begin{array}{c}
\sin \theta \cdot \cos \varphi \\
\sin \theta \cdot \sin \varphi \\
\cos \theta
\end{array}\right\}
\end{gathered}
$$

The half-spaces are used to determine whether point $\mathbf{P}_{\mathbf{i}}$ is below the plane $E$, defined by the normal vector $\overrightarrow{r_{2}}$ and base point $\mathbf{P}_{\mathbf{E}} \cdot \mathbf{P}_{\mathbf{i}}$ is tested against half-space (1), as shown in Figure 65, with Equations (4) and (5).

Figure 65: Half-space partitioning of a point cloud

$$
\boldsymbol{P}_{\boldsymbol{i}}-\boldsymbol{P}_{E}=\overrightarrow{\boldsymbol{d}}
$$




\subsubsection{Definition of stair-stepping and overhanging regions}

Each triangle can be analyzed whether the stair-stepping effect or overhanging happens. Figure 66 and Figure 67 show two different cases, where a triangle is identified as being in an overhang area or in a non-overhang area. An overhang cannot be printed without the support if the angle between the printing direction and the normal direction exceeds a certain threshold $(\alpha)$, as shown in Figure 68. Visual inspection of the upskin and downskin can be a powerful tool for analysis of the part geometry, as depicted in Figure 69.

Figure 66: Non-overhang (upskin) area
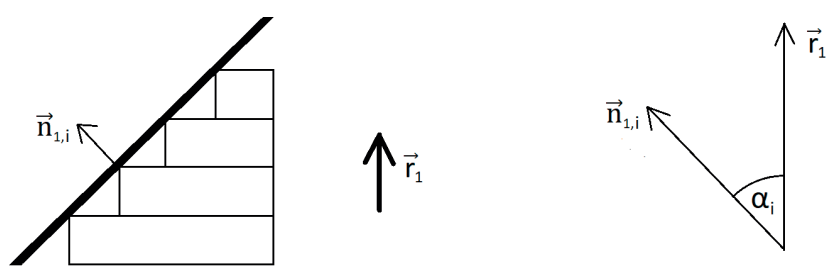

Figure 67: Overhang (downskin) area
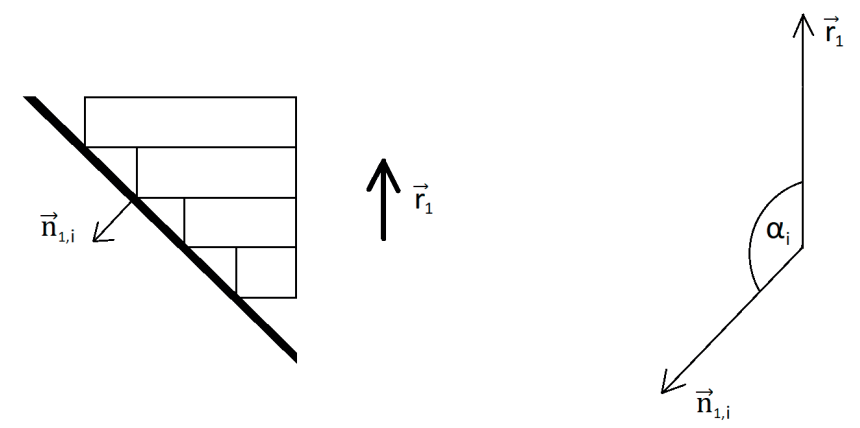

Figure 68: Overhang threshold

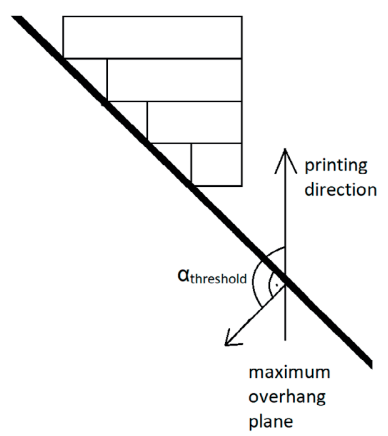


- Figure 69: Examples of overhanging and non-overhanging surfaces

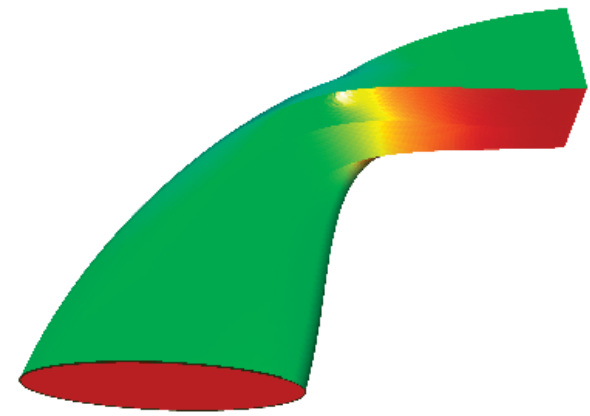

a) Downskin over 45 degrees (gradient from yellow to red)

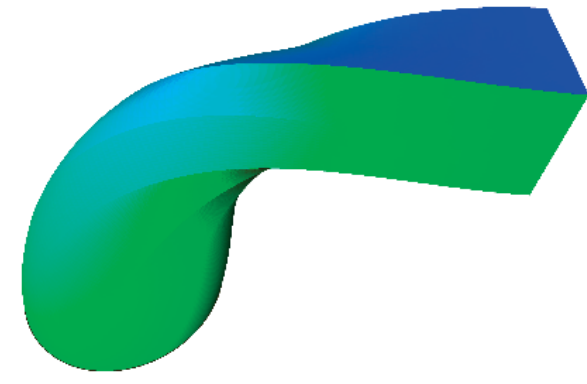

b) Upskin over 60 degrees (gradient from cyan to blue)

The layered deposition process works by depositing thin layers onto a previously deposited layer. Since deposition in the air is not possible, there is a limitation to the overhangs that can be created using this process. The capability of printing overhangs and its type is defined here by a threshold angle where 90 degrees means that there is a steep wall (no overhang) and $180^{\circ}$ means that a horizontal overhang is present (like the bottom side of a bridge).

$\alpha_{\text {threshold }}$ is the angle defined by the constraints of the process. Different parameters, such as the cooling fan speed, print speed and print temperature, can make a great contribution to the printable overhang angle size. For the given material and hardware, the maximum possible threshold angle can be defined to allow overhangs to some extent. It is obvious that 3D printing cannot happen in the air, so each subsequent printing layer can only slightly deviate from its predecessor when it comes to generating overhangs without support. When defining a threshold angle $\left(\alpha_{\text {threshold }}\right)$, computation can be constrained to secure overhang-relaxed slicing. 


\subsection{FULL-VOLUME DECOMPOSITION BASED ON HALF-SPACES}

Let's define the global error function $F_{1}$ for all the triangles in half-space (1) and, analogously, $F_{2}$ for half-space (2). Global error functions can be formulated in many ways. Let's assume the global error function is the total sum of all error functions for all triangles. The resulting global error function $F$ is a sum of $F_{1}$ and $F_{2}$, as presented in the set of equations below (6).

$$
\begin{aligned}
& F=F_{1}+F_{2} \\
& F_{1}=\sum_{\substack{n_{1} \\
n_{2}}} F_{1, i} \\
& F_{2}=\sum_{i=1}^{n_{2}} F_{2, i}
\end{aligned}
$$

As depicted in Figure 63, the goal of the optimal $E$ is to have $\overrightarrow{\mathbf{r}}_{\mathbf{1}}$ and $T_{1, i}$ comply with 3D printing process requirements, so $\overrightarrow{\mathbf{n}}_{\mathbf{1}, \mathbf{i}}$ (normal vector of triangle $T_{1, i}$ ) and $\overrightarrow{\mathbf{r}}_{\mathbf{1}}$ are either orthogonal or parallel to each other. The same applies to the pair $\overrightarrow{\mathbf{r}}_{2}$ and $T_{2, i}$.

An error function can be formulated to find the optimal $E$. To avoid the stair stepping effect coming from the slope of the triangle surface in relation to printing direction, the triangle should be either parallel or orthogonal to the printing direction, which can be formulated at the zero-error case for the error function for a given triangle and printing direction. Note, that $E$ defines the printing direction. The error function should aim to minimize the cusp height as defined in Equation 1 above which is based on triangle normal vector and printing direction. Further, overhang situations as defined in Figure 67 and Figure 68 with the respective threshold overhang angle, should be avoided. A penalty coefficient for such case helps to guide an error function to search for an optimal $E$ which avoids such overhang cases. At last, the triangles can be different size, such the size of a triangle should be a weight factor to calculate the sum of all errors for all triangles weighted by their size.

The above definition allows us to design an error function $F_{1, i}$ :

- If $\overrightarrow{\mathbf{n}}_{\mathbf{1}, \mathbf{i}}$ and $\overrightarrow{\mathbf{r}}_{\mathbf{1}}$ are parallel or almost parallel (within a given threshold), then error function $F_{1, i}$ equals 0 .

- If $\overrightarrow{\mathbf{n}}_{\mathbf{1}, \mathbf{i}}$ and $\overrightarrow{\mathbf{r}}_{\mathbf{1}}$ ar orthogonal (within a given threshold tolerance), then error function $F_{1, i}$ equals 00 .

- If neither of the conditions above are met, then there is a weighted error function, given in Equation (7), depending on the angle between $\overrightarrow{\mathbf{n}}_{\mathbf{1}, \mathbf{i}}$ and $\overrightarrow{\mathbf{r}}_{\mathbf{1}}$. 


$$
\begin{gathered}
\alpha_{1, i}<\alpha_{\text {threshold }} \Rightarrow F_{1, i}=\alpha_{1, i} A_{1, i} \\
\alpha_{1, i}>\alpha_{\text {threshold }} \Rightarrow F_{1, i}=\left(\alpha_{\text {threshold }}+K\left(\alpha_{1, i}-\alpha_{\text {threshold }}\right)\right) A_{1, i}
\end{gathered}
$$

, where

$K$ is a penalty coefficient, which controls the weight of deviation from the allowed threshold angle of overhangs.

$A_{1, i}$ is the area of the triangle.

A split plane with an arbitrary orientation will generate an error map in which each triangle can be assigned a certain normalized error function without considering the triangle areas, as shown in Figure 70. This example has the penalty coefficient $K=10$ for angles between the plane orientation and triangle normal vectors exceeding 45 degrees.

Figure 70: Visualization of the normalized error function (for unit triangle area)
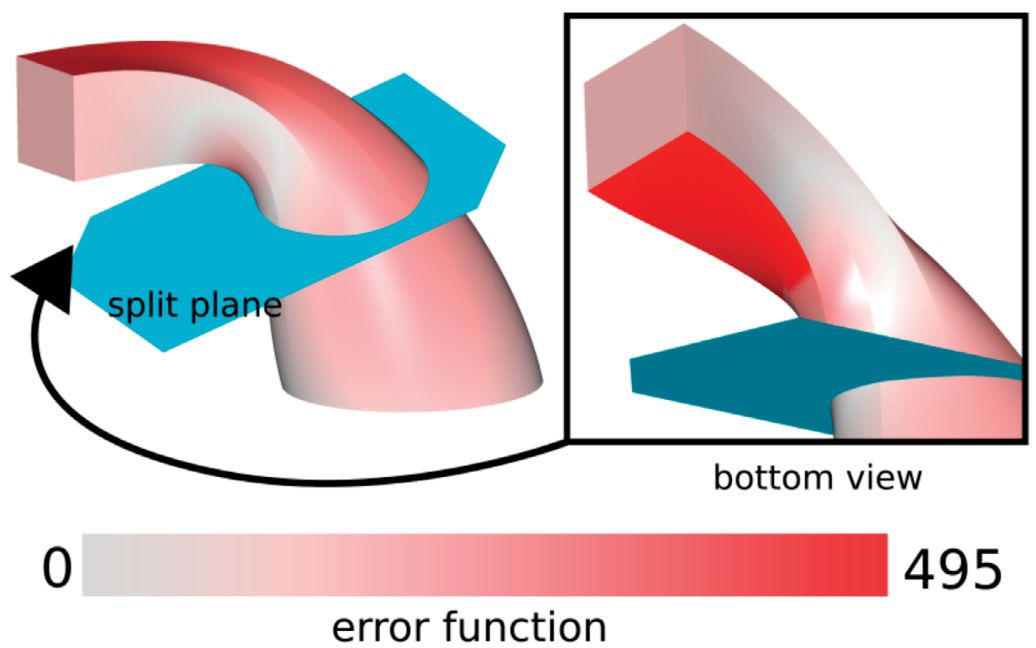

Note that plane $E$ determines whether a triangle belongs to half-space (1) or (2). In cases where plane $E$ splits a triangle, we assume that such a split creates two sets of triangles, each of which can be assigned to one of the half-spaces, as shown in Figure 71. Each triangle intersected by plane $E$ must be subdivided into four smaller triangles. One newly generated triangle will be in one half-space, while the three other triangles will be in the other half-space. 
Figure 71: Split of triangles intersected by the half-plane $E$

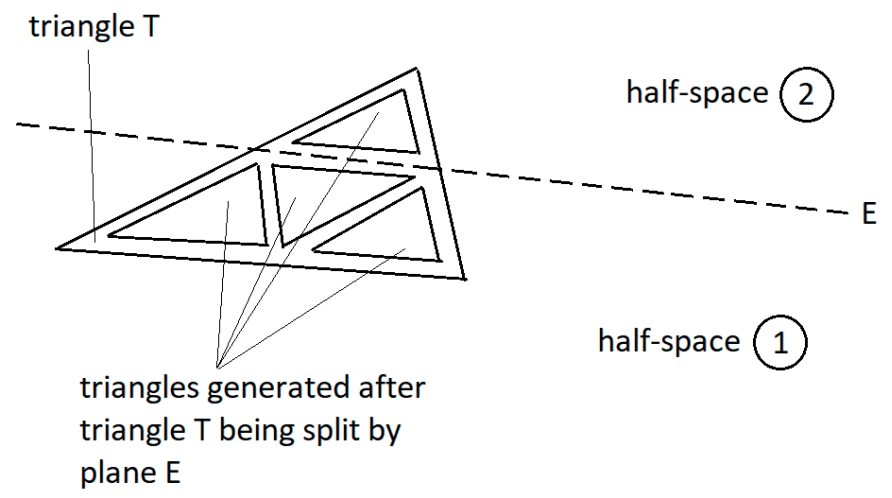

Our global error function $F$ is a function of plane $E$. The goal is to minimize the total error, as given in Equation (8).

$$
\operatorname{minimize}(F(E(\boldsymbol{S}, \varphi, \theta))
$$

, where

$S$ is a position defining plane $E^{\prime} s$ position;

$\varphi$ and $\theta$ are the Euler angles defining plane $E$ 's orientation.

Since plane $E$ must intersect the part geometry, the search space of $\boldsymbol{S}$ is limited to the volume of the part geometry. However, it can be computationally expensive to check whether a point is inside a closed volume. Therefore, an optimization is suggested: the search space of $\boldsymbol{S}$ is extended to the axis-aligned bounding box (AABB), a cuboid bounding the volume of the part along the coordinate axes. An AABB enclosing the part defined in the coordinate system $x, y$, $z$ with the origin $O$ has eight corner points $C_{j}$, as presented in Equation (9). A 2D perspective of an $A A B B$ is shown in 72 .

Figure 72: Definition of an axis-aligned bounding box enclosing the part geometry

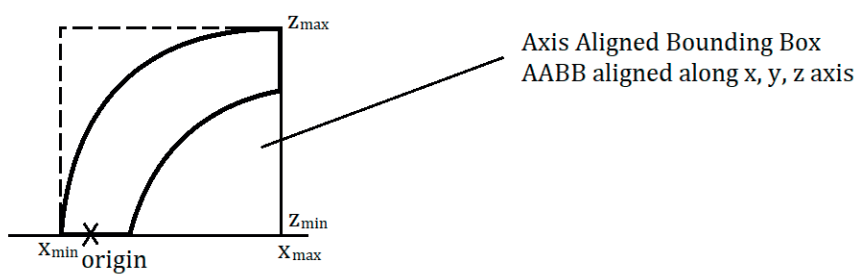




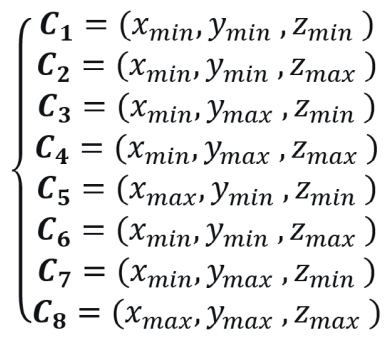

The search domain $d$ representing an AABB is defined in Equation (10). $d_{\text {min }}$ and $d_{\text {max }}$ define the boundary points of the search algorithm. For instance, they can define the endpoints of an AABB edge or diagonal, while the search is run by iterating points on the line segment defined by $d_{\min }$ and $d_{\max }$.

$$
d \in\left[d_{\min }, d_{\max }\right]_{]}
$$

, where

$$
d_{\min } \text { and } d_{\max } \text { define AABB extent. }
$$

Equation (8) can be formulated as the three-dimensional problem given in Equation (11).

$$
\operatorname{minimize}(F(d, \varphi, \theta)
$$

subject to $\varphi \in\left[0^{\circ}, 360^{\circ}\right]\left|\theta \in\left[0^{\circ}, 180^{\circ}\right]\right| d \in\left[d_{\min }, d_{\max }\right]$

For an angular step of 1 degree to variate $\varphi$ and $\theta$ along with 1000 points within the domain $d$, the number of possible combinations is the product of $360^{\star} 180^{\star} 1000=64,800,000$. This means that a brute-force algorithm needs to calculate the error function nearly 65 million times just for a subdivision of the part in two subregions.

\subsubsection{Point cloud approximation of meshes}

To calculate the error function for a given set of $(d, \varphi, \theta)$, the entire triangle mesh needs to undergo a Boolean split in 3D space, which is a costly operation that can take between a few milliseconds and few seconds for each calculation, depending on the number of triangles that make up the shape. Even in a best-case scenario of 1 millisecond, this operation would lead to 65,000 seconds of calculation time, which is around 1,100 minutes or around 20 hours. Furthermore, the development of a 3D mesh Boolean method requires sophisticated implementation efforts, while the concerns about numerical stability are present in cases where a Boolean operation on 3D mesh requires a triangle-to-triangle intersection calculation, which is numerically difficult when the triangles are almost parallel to each other. The computers use single or double precision to carry out these calculations, and almost parallel triangles and their intersection require unlimited precision in the calculation. Since this is not possible with 
state-of-the-art computers, using numerical algorithms to solve such problems is considered very challenging as it is difficult to get reliable results with limited-precision calculations.

Both numerical stability concerns and the cost of running computationally expensive 3D mesh Boolean operations can be avoided by approximating the triangle $T_{i}$ by a number of points $\boldsymbol{P}_{i, j}$, as shown in Figure 73. Instead of operating triangles, the algorithm can perform some quick tests on a point cloud.

Figure 73: Points to approximate a triangle

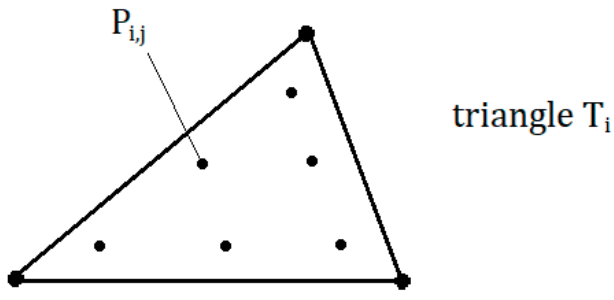

If there are $n$ points of $\boldsymbol{P}_{i, j}$ evenly distributed over the surface of a triangle, each point can be associated to an equal fraction of the triangle's surface area $A_{i}^{\prime}$, as given in Equation (12).

$$
A_{i}^{\prime}=\frac{A_{i}}{n}
$$

Basically, we do not use the triangle mesh anymore. We use points $\boldsymbol{P}_{i, j}$ instead, where each $\boldsymbol{P}_{i, j}$ has an associated normal $\overrightarrow{\boldsymbol{n}_{\boldsymbol{\imath}} \boldsymbol{j}}$, which is still the triangle normal coming from $\overrightarrow{\boldsymbol{n}_{\boldsymbol{\imath}}}$ of the triangle $T_{i}$, from which $\boldsymbol{P}_{i, j}$ is derived. Furthermore, $A_{i}^{\prime}$, which defines the zone area for every $\boldsymbol{P}_{i, j}$ comes from triangle $T_{i}$ 's area $A_{i}$ divided by the number of inserted points $\boldsymbol{P}_{i, j}$. Since mesh triangles vary in size, it is not consistent to define the number of points as constant for all triangles. Instead of $n$, a user-provided global value of $\Delta A$, which defines the target for area size for inserted points, should be used. The actual $A_{i}^{\prime}$ is unlikely to meet $\Delta A$ because the areas of triangles tend to vary and $n_{i}$, the number of inserted points per triangle, is an integer. Thus, the number must be rounded to the nearest integer, but it cannot be less than 0 , as demonstrated in Equation (13).

$$
n_{i}=\max \left(1,\left[\frac{A_{i}}{\Delta A}\right]\right)
$$

Therefore, Equation (7) can be rewritten as Equation (14). This approximation simplifies the task of calculating the error function $F$. 


$$
K\left(\alpha_{1, i}-\alpha_{\text {threshold }}\right) A_{1, i} n_{i}
$$

Determining whether a point $\boldsymbol{P}_{i, j}$ belongs to half-space (1) or (2) can now be done very quickly using the plane equation previously explained in Figure 65 and Equation (4).

\subsubsection{Multi-plane decomposition}

As described in the above algorithm, the problem of finding a plane $E$ to split the space into two subsections is transformed to a global error function with three degrees of freedom. Triangles are approximated by simpler point objects with the respective triangle normal vector assigned to derived points. This subsection extends the algorithm, allowing it to search and find multiple subsequent half-spaces to help with the processing of more complex shapes using $3+2$ axis AM. Note that although the above-described algorithm is limited to one half-space $E$, it is believed to have the potential to be extended to multiple subsequent half-space calculations for multi-axis 3D printing, as shown in Figure 74, which depicts two planes, $E$ and $E^{\prime}$.

Figure 74: Multiple subsections for $3+2$ axis printing

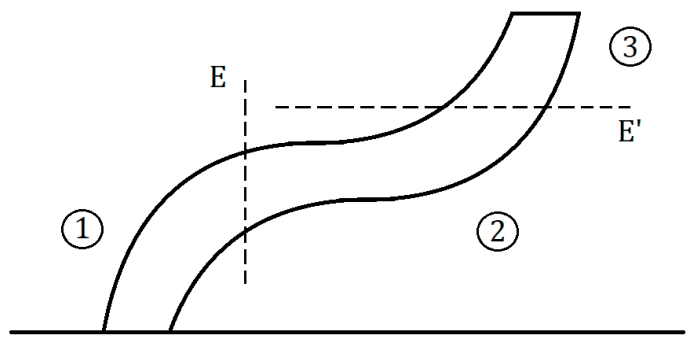

Finding the minimum value of the error function for all planes ( $E$, $E^{\prime}$, etc.) is a computationally demanding task. In the case of a single split-plane $E$, there were three unknowns. For $N$ subsequent planes, the number of unknowns becomes $N$ times three. In other words, the error function $F$, which was initially three-dimensional, becomes $N$ times three-dimensional. The fact that the number of planes is also unknown presents another challenge.

The following proposed algorithm reduces the complexity, taking it back to a three-dimensional problem. The initially proposed algorithm for splitting the space into two half-spaces will be used. However, all the points located from the split-plane $E$ (with the distance from $E$ exceeding a given threshold distance) will not be relevant to the error function. Once the first plane $E$ is known, the algorithm is applied again using the remaining points. Figure 75 shows a part split into three subspaces (1), (2), and (3)). First, plane $E_{(1),(2)}$ was generated. Then, the next plane $E_{(2), 3}$ was generated without considering points in the half-space (1). 
Figure 75: Notation for multiple planes

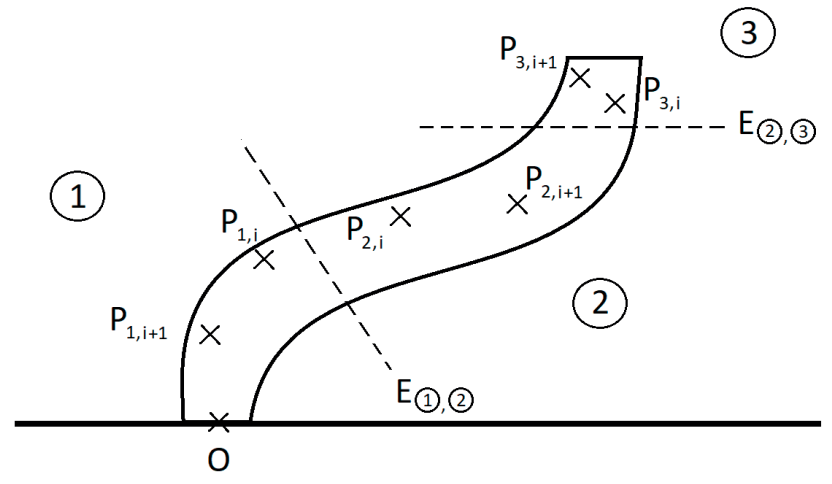

Now we have multiple error functions-one for each split plane. $F_{(1),(2)}$ is the error function for plane $E_{(1),(2)}$, and, accordingly, $F_{(2,3)}$ is the error function for plane $E_{(2), 3}$.

As already defined in the initial algorithm from Equation (6), the global error function $F=F_{1}+F_{2}$ must be modified to consider the additional split plane. In the multi-plane partitioning, we will ignore any $F_{1, i}$ in each error function calculation for the corresponding $\boldsymbol{P}_{1, i}$ in order to calculate any $F_{1, i}$ that is far away from the previous split plane.

\subsubsection{Search for a split-plane position}

The search for a split-plane position starts with the initialization of the initial plane position. The split plane number 0 is defined as $E_{0}$ and shown in Figure 76 . The initial position is at the origin of the coordinate system, which is located on the table. Plane orientation is collinear to $\overrightarrow{\mathbf{z}(\mathbf{0}, \mathbf{0}, \mathbf{1})}$, which is defined by the table surface orientation.

Figure 76: Definition of $E_{0}$

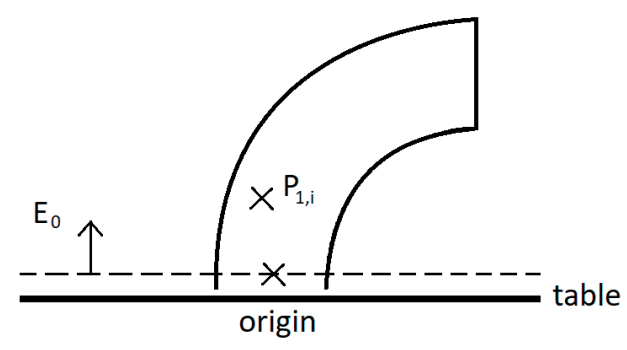


Since printing starts from the table and follows the initial printing direction $\overrightarrow{\boldsymbol{Z}(\mathbf{0}, \mathbf{0}, \mathbf{1})}$, the search for the points for the next split plane is performed by iterating the points that are above the initial plane (i.e., the table). The search space is not unlimited. Each candidate point is examined for whether it is close enough to be considered, as shown in Equation (15). Equation (15) checks whether the distance from the origin to the candidate point in the $z$ direction is less than the user-defined value $d_{1, \max }$.

$$
\left(\overrightarrow{\boldsymbol{P}_{1, \boldsymbol{\imath}}}-\overrightarrow{\text { Orıgın }}\right) \overrightarrow{\mathbf{z}}<d_{1, \max }
$$

, where

$d_{1, \text { max }}$ is user-defined input for the upper limit of the size of the search space.

In 3D printing layer thickness, $l$ is usually known. For printing with a constant layer thickness, planning must avoid printing layers of lower or increased thickness. Therefore, the number of layers $\left(n_{\text {layers }}\right)$ should be given as a number. Accordingly, user input for $d_{1, \max }$ can be designed by letting the user provide the layer thickness and the maximum number of layers to be used for each subdivision, as demonstrated in Equation (16). This will reduce our search to a finite number of layers.

$$
d_{1, \max }=l \cdot n_{\text {layers }}
$$

, where

$l$ is the layer thickness;

$n_{\text {layers }}$ is the maximal number of layers in the search space.

In addition, the user can provide $n_{\text {layers }}$ as a single number of layers for a globally defined distance $\left(d_{\text {global,max }}\right)$ for all half-spaces, as presented in Equation (17).

$$
d_{\text {global,max }}=l \cdot n_{\text {layers }}
$$

Analogously, the same approach can be applied to the next plane calculation. Figure 77 depicts plane $E_{1}$ at the point $S$. Like Equation (15), the distance from a candidate point $\boldsymbol{P}_{\mathbf{1}, i}$ is considered for the skip test, as shown in Equation (18). If the distance is less than the global upper limit $\left(d_{\text {global,max }}\right)$, then we consider $\boldsymbol{P}_{2, i}$ as "close," so it can be taken into account for the error function. 
Figure 77: Definition of $E_{1}$

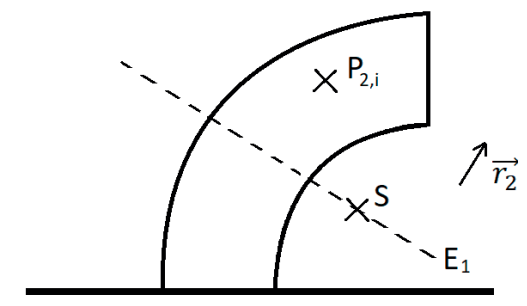

$$
\left(\overrightarrow{\boldsymbol{P}_{2, \boldsymbol{l}}}-\overrightarrow{\boldsymbol{S}}\right) \overrightarrow{\boldsymbol{r}_{2}}<d_{\text {global,max }}
$$

\subsubsection{Algorithm summary}

To summarize the above-described multi-step approach to finding a series of $3+2$ axis AM directions, the following can be stated:

- The user needs to provide a meaningful global number of layers for printing directions. For example, if the user decides to set this value to 10 layers, then the $3+2$ axis printing direction will be changed after printing these 10 layers.

- The user needs to define the relative penalty for allowing overhangs that require support structures. Currently, a big penalty number $(K)$, for example 10, defines the relative weight in error function of a point being in an overhang versus a point being printed under a certain angle.

- Triangles are approximated by points. The number of points generated is related to $\Delta A$, which is the subarea upon which a triangle is split into multiple points.

- Iteration increment values for $\varphi, \theta$ and $d$ are also user inputs.

To simplify the procedure, the following user input is preferred:

- Instead of defining an arbitrary $d_{\text {global,max }}$ from Equation (17), define globally the maximal number of layers for each $3+2$ axis printing direction in relation to the size of machine working space as a function of $z$ height, as demonstrated in Equation (19).

$$
n_{\max z \text { layers }}=\frac{Z_{\max }-Z_{\min }}{l}
$$

, where

$z_{\max }$ and $z_{\min }$ are the vertical limits of the working space.

This means that if the printing direction remains $z$-direction, the maximum $z$ height possible for the 3D printer hardware and the layer thickness $(I)$ are used to calculate the maximum number of possible $z$ layers. 
- It is helpful for the user to provide $n_{\text {desired repositioning }}$, which is the desired number of $3+2$ axis steps. For example, the frequency of repositioning (subdivisions) the printing directions can be set to 20 . This variable can be used to set $n_{\text {layers }}$ for each step, as shown in Equation (20).

$$
n_{\text {layers }}=\frac{n_{\text {max z layers }}}{n_{\text {desired repositioning }}}
$$

Note that due to tilting, the local printing direction will not be on the $z$-axis, so the resulting repositioning steps can be greater. Still, it will remain within the same order of magnitude of the user-provided desired number of repositioning directions.

- Approximating the triangle by points requires the user to set the $(\Delta A)$ area size. Since the precision of the result is related to layer thickness $(I)$, it is suggested to use the value of $l$ for setting $\Delta A$.

- Iteration steps require user input. Since 3D printing typically accepts overhang angles up to 45 degrees, the iteration angle steps for $\varphi$ and $\theta$ can be set so that the integer multiplication could be exactly 90 degrees and 135 degrees (90 degrees +45 degrees). For example, a step of one degree or five degrees.

- Additionally, in order to speed up calculation, the user can restrict the defined domain of $\theta$ to avoid collision with the table or other kinematical elements of the hardware. For example, $\theta>90$ degrees will likely create collisions. Similar constraints may further reduce the search domain for the minimization problem.

The variable $d$ is the distance range to iterate. It aims to find the basse point $\boldsymbol{S}$ of the split plane, as described above. For the same purpose of calculating varying $\boldsymbol{S}_{\text {points, we can define the }}$ distance parameter $\boldsymbol{d}$ as the distance of $\boldsymbol{S}$ from the previous printing plane in the previous printing direction. As a result, the necessary iteration of $d$ will be restricted to the range given in Equation (21). In other words, we would allow a new split plane so that the printing up to this new calculated split plane would be at least one printing layer or at most $n_{\text {layers }}$.

$$
1 \cdot l \leq d \leq n_{\text {layers }} \cdot l
$$

, where

$l$ is the layer thickness. 


\subsubsection{Algorithm pseudo-code}

Eventually, the algorithm can be presented by the pseudo-code in Figure 78.

Figure 78: Algorithm's pseudo-code

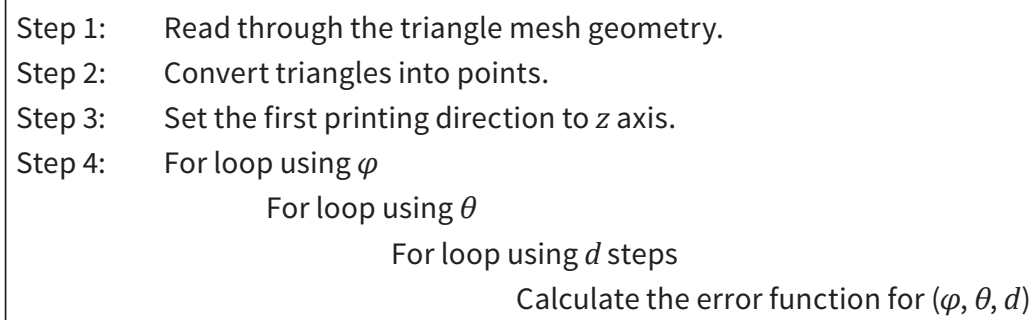

Step 5: Choose $\varphi, \theta, d$ with minimal error function value, then calculate the current printing direction and split plane. Remove all points in the half-space below the split plane

Step 6: $\quad$ Stop if no points are left.

Step 7: $\quad$ Set the previous printing direction as the current printing direction.

Step 8: $\quad$ Go to Step 4.

\subsubsection{Algorithm implementation}

Figure 79a shows how the graphic user interface (GUI) is implemented in the dedicated software developed for this research. The graphic user interface consists of several areas responsible for controlling parameters and visualizing geometries. Figure $79 \mathrm{~b}$ depicts the GUI used to control all parameters of the application. The colorful stripes show how the algorithm splits the part into subsections with the goal of reducing surface roughness and avoiding overhangs beyond the threshold value that would require supports. The stripes present on each subsection show the printing direction is orthogonal to the bottom of each subsection.

Figure 80 shows a user interface used to control the parameters of the decomposition algorithm. The list of parameters includes the angular step to search for optimal plane orientations, the threshold angle for overhangs, the penalty coefficient $K$ on overhanging points, and the threshold angle on triangles accepted as parallel to the slicing direction. Additionally, two parameters are defined. The desired number of slices sets the target number of slices for the algorithm to start with. The desired number of slices defines the search extent of the size of decomposition; a "penalty for small slices" means pieces that are too small will not be produced. Instead, they will be merged with larger neighboring chunks. Desired slices are used to set the look-ahead $d_{\text {global,max }}(z$ height of the geometry divided by the number of desired slices). 
- Figure 79: Screenshots of the software application with the implemented algorithm

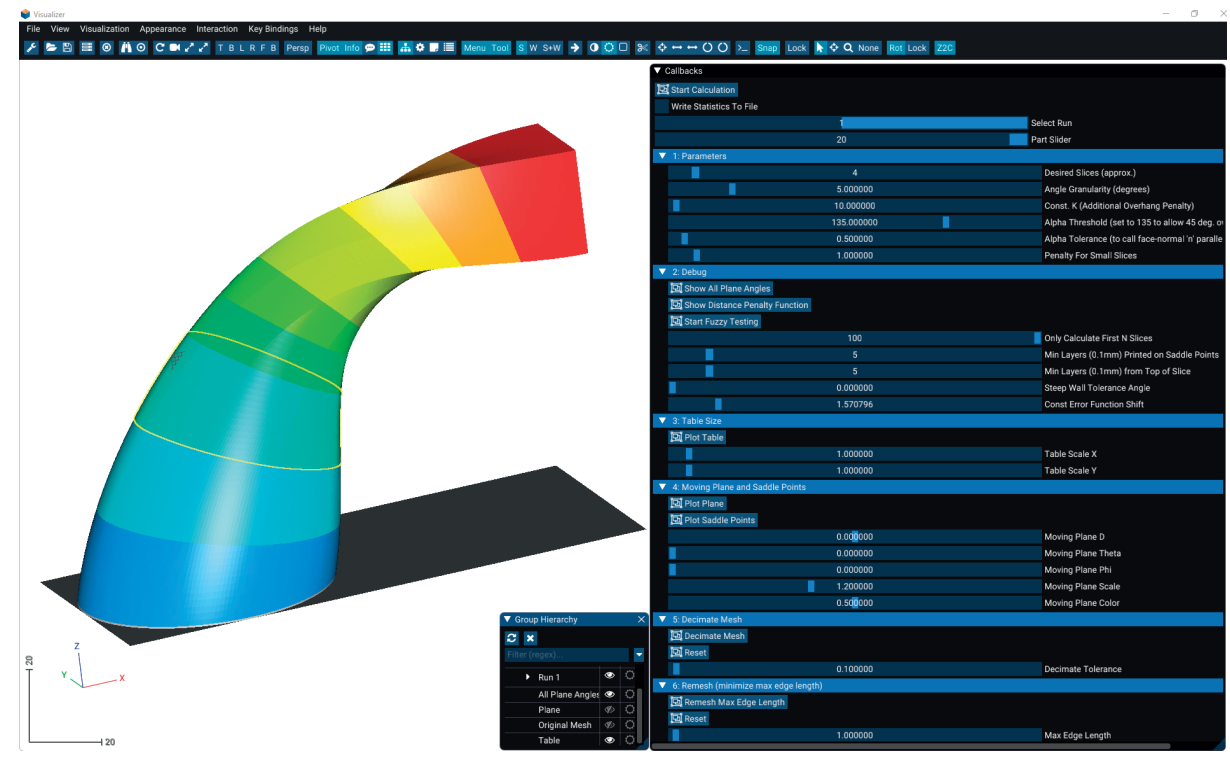

a) Main window of the software application for volume decomposition

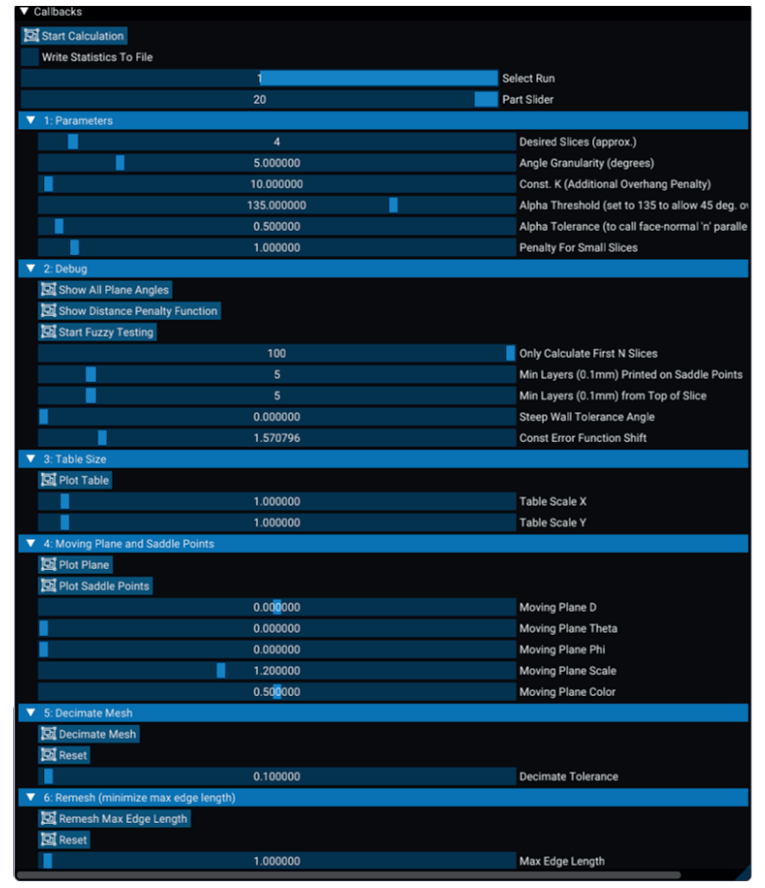

b) GUI panel to control parameters of software application 
It is important to note that the user provides the parameters that decide the calculation accuracy (angle steps for iteration), which is a trade-off between the accuracy required to find optimum planes and the total calculation time. The user does not need to provide any parametrization for the geometry shape, which proves that the algorithm can deal with random complexity.

Figure 80: GUI panel with the input parameters

\begin{tabular}{|c|c|c|c|c|}
\hline \multicolumn{5}{|c|}{ 1: Parameters } \\
\hline \multirow[t]{2}{*}{ 口 } & & 4 & & Desired Slices (approx.) \\
\hline & ] & 5.000000 & & Angle Granularity (degrees) \\
\hline \multirow[t]{2}{*}{ 口 } & & 10.000000 & & Const. K (Additional Overhang Penalty) \\
\hline & & 135.000000 & D & Alpha Threshold (set to 135 to allow 45 deg. overhang) \\
\hline प & & 0.500000 & & Alpha Tolerance (to call face-normal 'n' parallel to print dir. 'r') \\
\hline 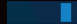 & & 1.000000 & & Penalty For Small Slices \\
\hline
\end{tabular}

\subsubsection{Decomposition examples}

Since no affordable $3+2$ axis FDM hardware is commercially available for experimental validation at the time of this thesis being written, the proof is provided by using geometric results, where split planes and their according subsections are highlighted with toggling colors. The construction of a $3+2$ FDM printer is foreseen as future research work.

For the sake of easy implementation and extending the algorithm toward the decomposing of branching geometries (Y-like), the search algorithm has been reversed to search from the top of the part to the table. Since the search route aims to minimize the global value of the error function, other directions of search may still result in slightly different results, but it should not differ too much.

For a given part, two different parameters are presented. The first parameter was the number of desired slices (1,2, 3, 4, and 5); this parameter defined the look-ahead distance that was used by the search algorithm to evaluate the error function value for the current decomposition volume. The smaller value meant a large distance with, presumably, a larger "thickness" to the subsection. The second parameter was the angular granularity (one and five degrees), which defined the angular steps for iterating the orientation of the split planes. Smaller angular steps increase the number of iterations and, therefore, increase the computation time.

Figure 81 to Figure 85 show 10 snapshots of the decomposition with all the possible combinations of the five different numbers of slices and two options for angular granularity. 
- Figure 81: Decomposition with desired slices $=1$

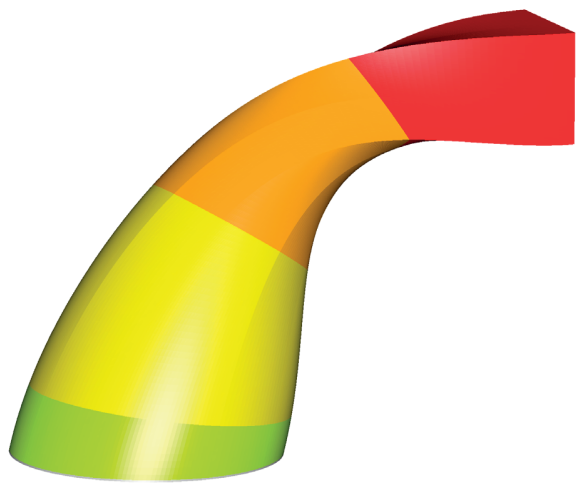

a) Angle granularity of one degree

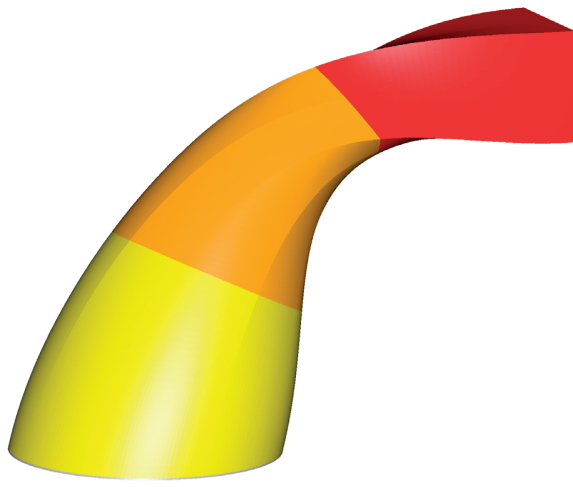

b) Angle granularity of five degrees

Figure 82: Decomposition with desired slices $=2$

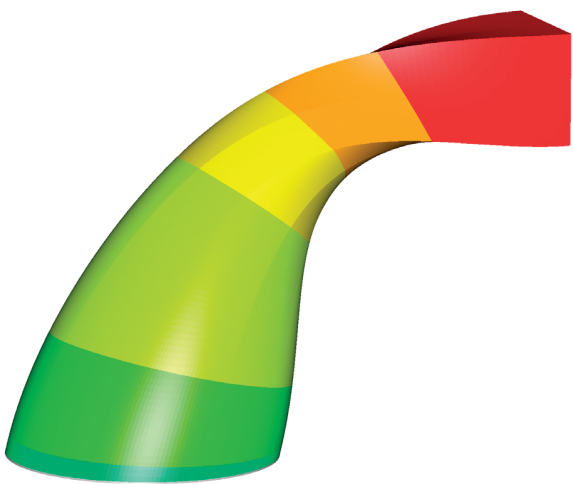

a) Angle granularity of one degree

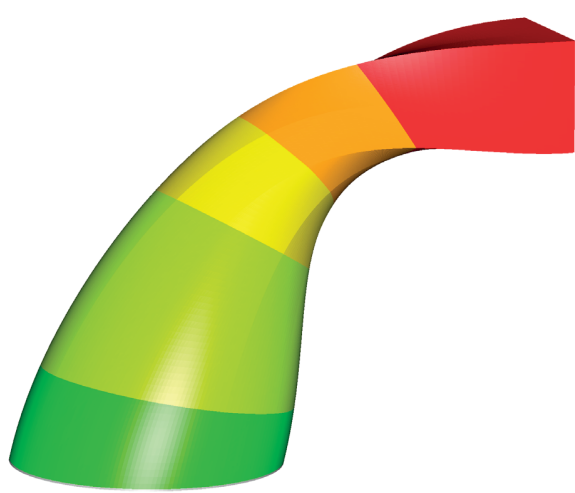

b) Angle granularity of five degrees

Figure 83: Decomposition with desired slices $=3$

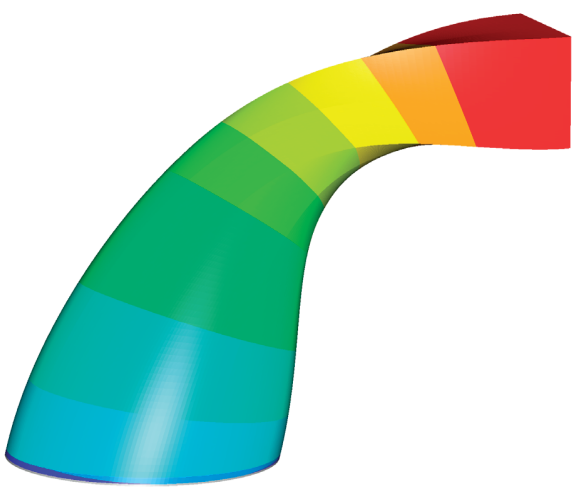

a) Angle granularity of one degree

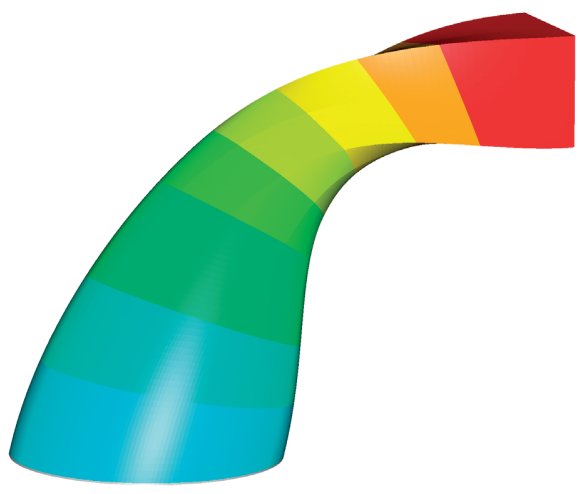

b) Angle granularity of five degrees 
Figure 84: Decomposition with desired slices $=4$

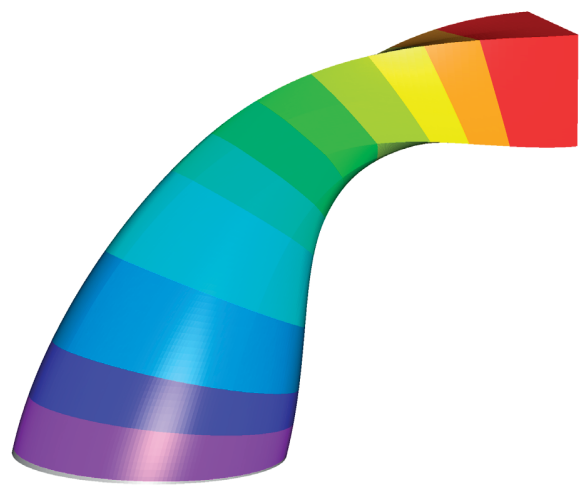

a) Angle granularity of one degree

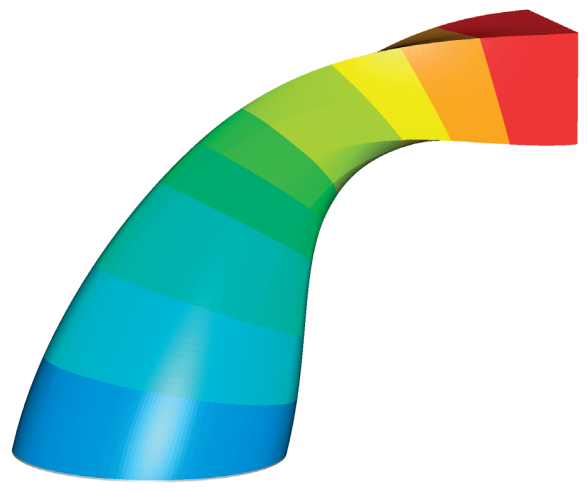

b) Angle granularity of five degrees

Figure 85: Decomposition with desired slices $=5$

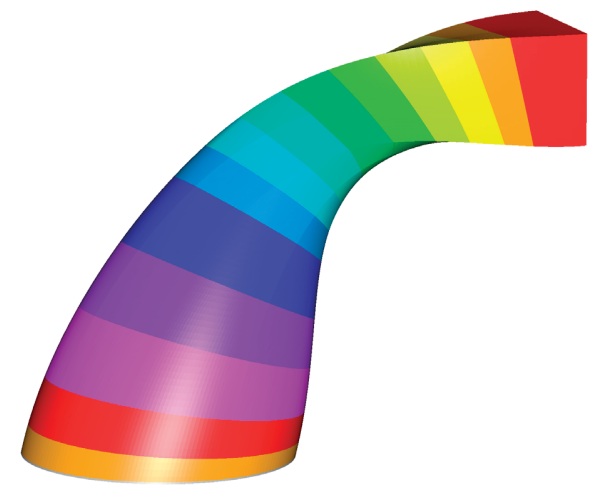

a) Angle granularity of one degree

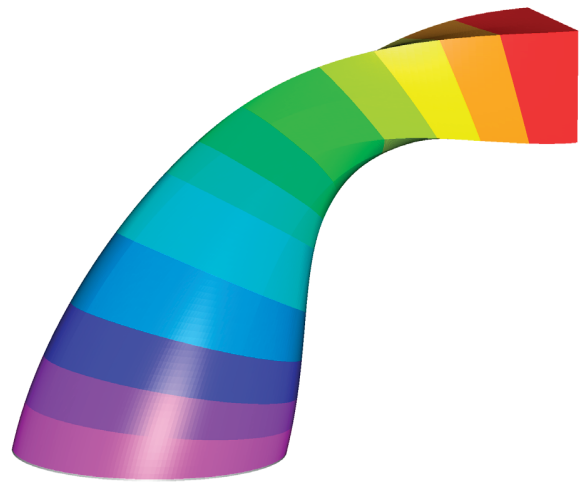

b) angle granularity of five degrees

It is noticeable that the smaller granularity (one degree) resulted in a larger number of subsections. Also, the difference in the decomposition with five degrees of granularity can be spotted as a relatively small chunk attached to the table. Due to the reverse direction of the algorithm search, this piece of geometry is processed last. The reverse direction of the search was selected as an approach in order to consider the branching of geometry primitives in T-like structures. This will be discussed in greater detail in section 5.2. In the case of five degrees of granularity, this subsection cannot be processed to fulfill the threshold criteria with the given angular step. Thus, it is attached to the previous one.

In addition, the global error function can be evaluated. The global error function sums up the error functions of all individual slices. Figure 86 shows two graphs presenting how the global error function changes depending on the number of desired slices and, therefore, on the look- 
ahead distance, which decreases while the number of desired slices increases. The general trend shows that the global error function tends to decrease with the decreased look-ahead distance. This can be explained by applying smaller subsections that are better aligned to the surface curvature to produce a smaller error. However, the function is not always downward monotone. It does spring back at the right side of the charts. It seems that the reverse direction in the trend was due to the discrete nature of the surface representation. The algorithm iterates over non-smooth triangulated surfaces with discrete steps, which can almost never meet an optimum. Thus, these upward variations in the graphs (less than one percent) can be attributed to numerical issues during computation.

Figure 86: Global error function for different trials

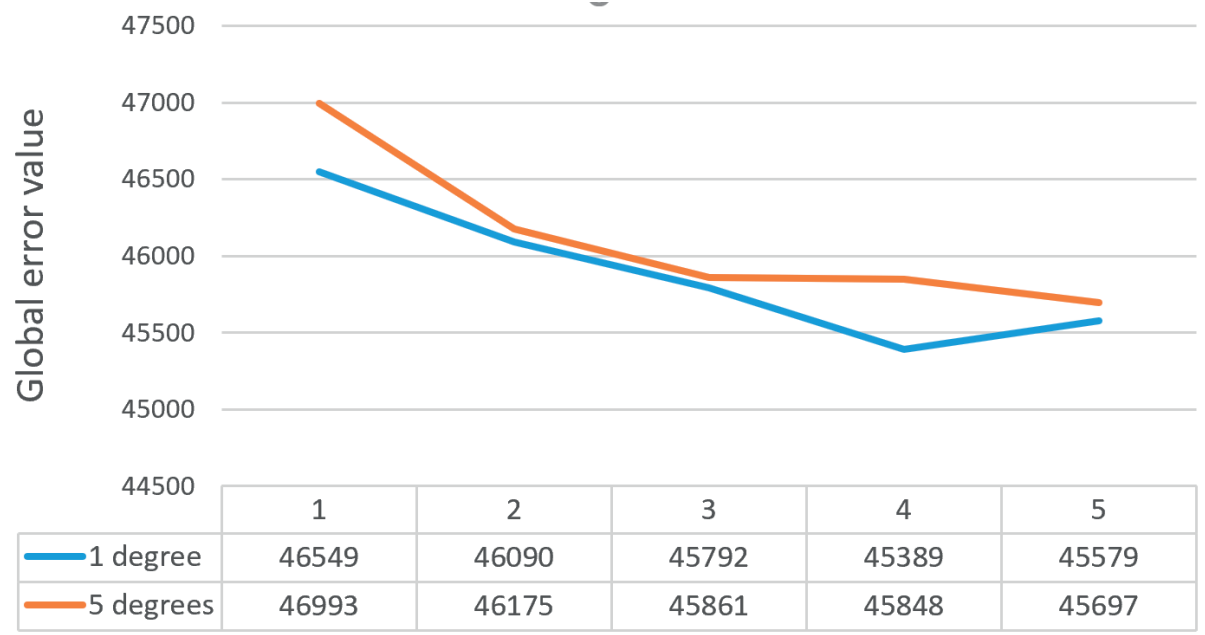

Number of desired slices

Understanding the global error function is not intuitive. The value of the global error function represents some metrics that cannot be directly mapped onto the existing measures describing surface quality. In addition to the global error function, the distribution of the scallop/cusp heights, derived in Equation (1) and presented in Figure 62, has been elaborated to assess the stair-stepping effect. The scallop height depends on the layer thickness and the orientation of the surface relative to the build direction. For the sake of simplicity, layer thickness can be omitted, and the stair-stepping cusp height can be represented as a fraction of the layer height. Figure 87 to Figure 96 show that the distribution of different cusp heights changes with the increase in the number of partitions in the test part. All charts share the "red" histogram of the cusp distribution of the test parts without any subdivision. Then, the number of partitions increases from four to 32. In Figure 87, 20 baskets of cusp heights with a step of five percent of 
the layer thickness are depicted with black bars. In the consequent illustrations (Figure 88 and Figure 96), grey bars depicting a histogram of the previous partitioning have been added in order to improve comprehension of the presented information. As demonstrated, applying the decomposition algorithm progressively decreases high-magnitude scallops. Four partitions have been enough to eliminate cusps above 65 percent of the layer thickness. Increasing the number of partitions to 32 helped to push this value down to 40 percent of the layer thickness. Four partitions led to a distribution skewed to the right side, while the larger numbers of partitions showed no clear trend. Additionally, the average cusp height decreased progressively from 0.377 for the non-partitioned, with a local bump at 26 partitions, as shown in Figure 97.

Figure 87: Distribution of cusp sizes for the part consisting of four partitions

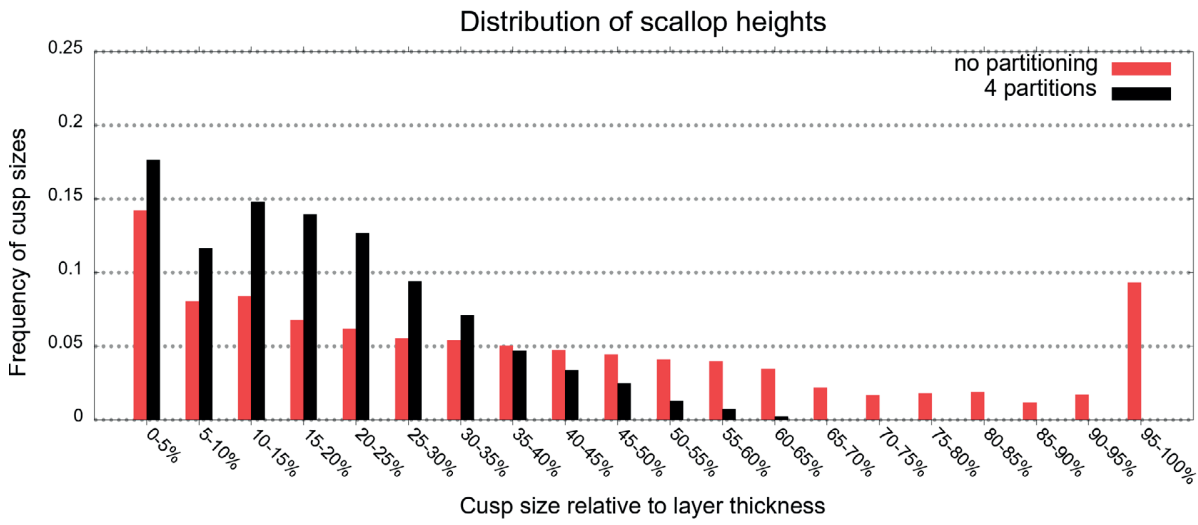

Figure 88: Distribution of cusp sizes for the part consisting of seven partitions

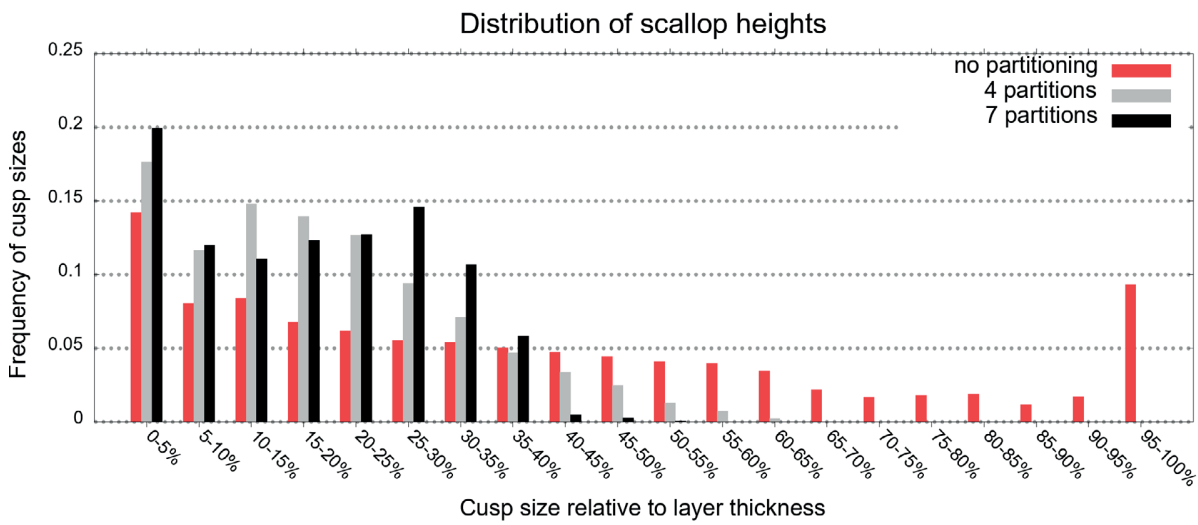


Figure 89: Distribution of cusp sizes for the part consisting of nine partitions

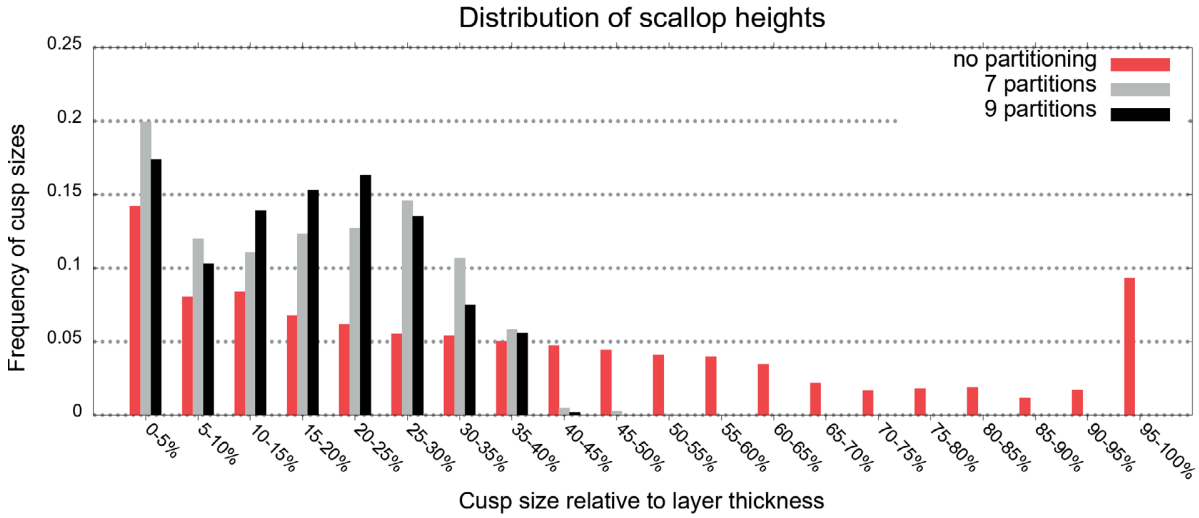

Figure 90: Distribution of cusp sizes for the part consisting of 13 partitions

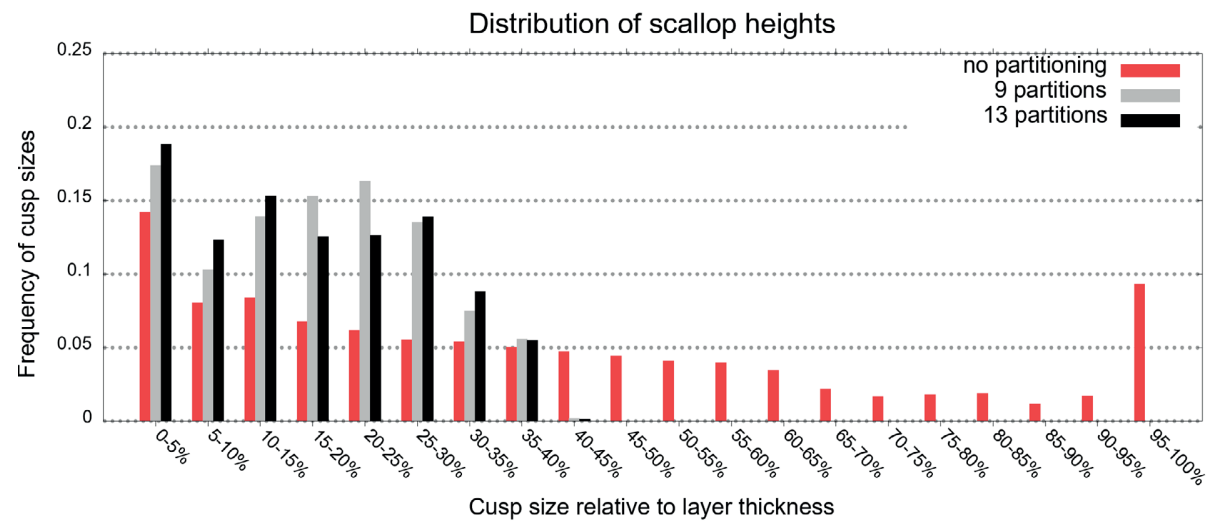

Figure 91: Distribution of cusp sizes for the part consisting of 16 partitions

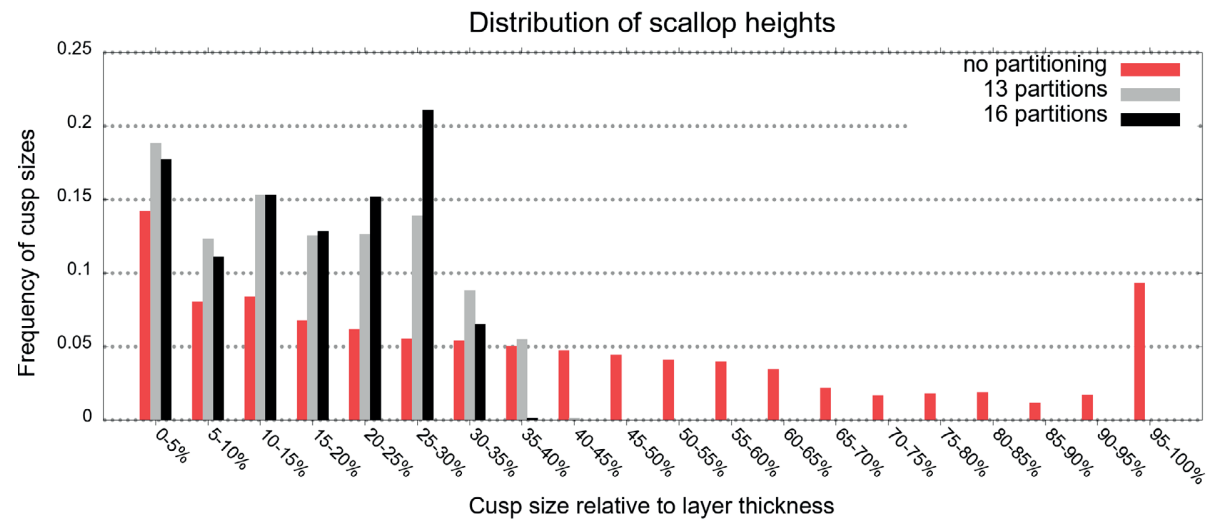


Figure 92: Distribution of cusp sizes for the part consisting of 18 partitions

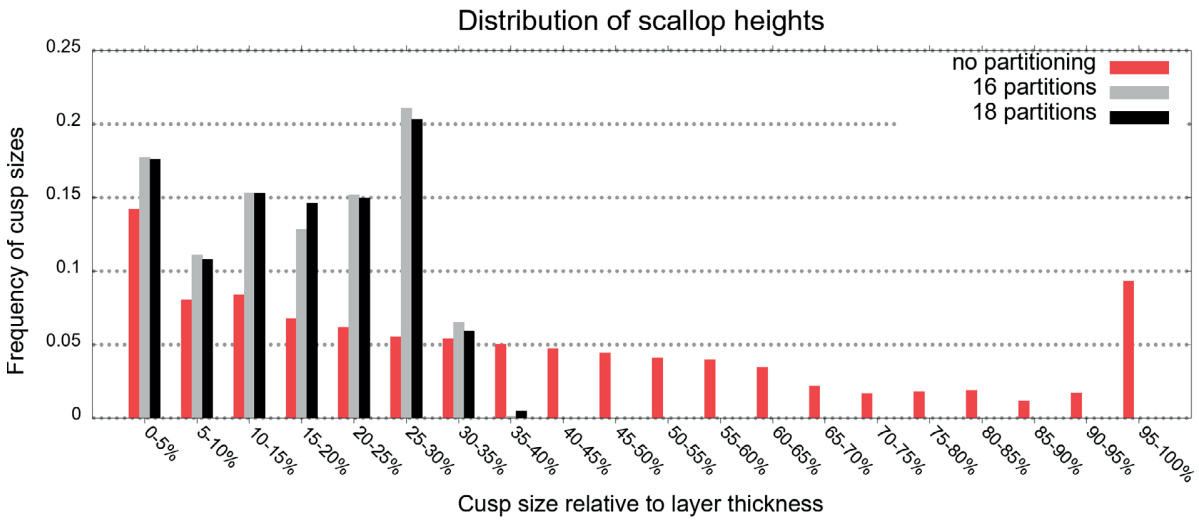

Figure 93: Distribution of cusp sizes for the part consisting of 21 partitions

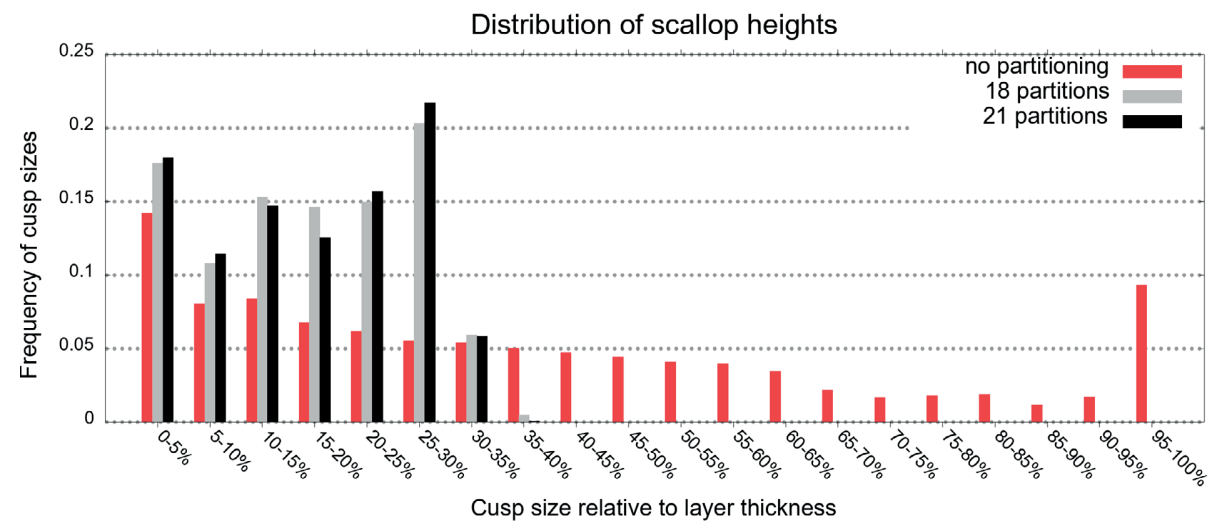

Figure 94: Distribution of cusp sizes for the part consisting of 24 partitions

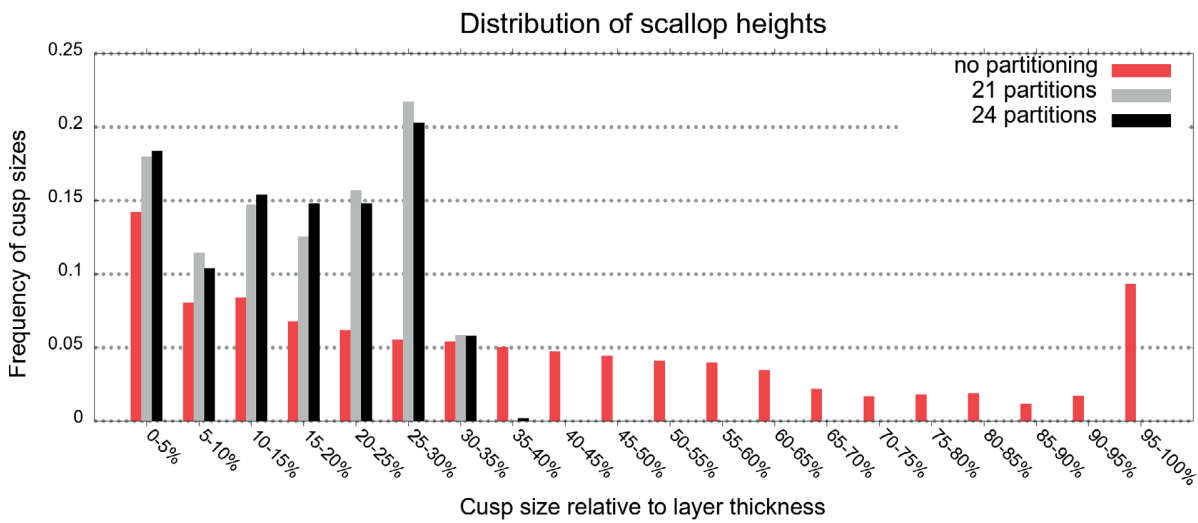


- Figure 95: Distribution of cusp sizes for the part consisting of 26 partitions

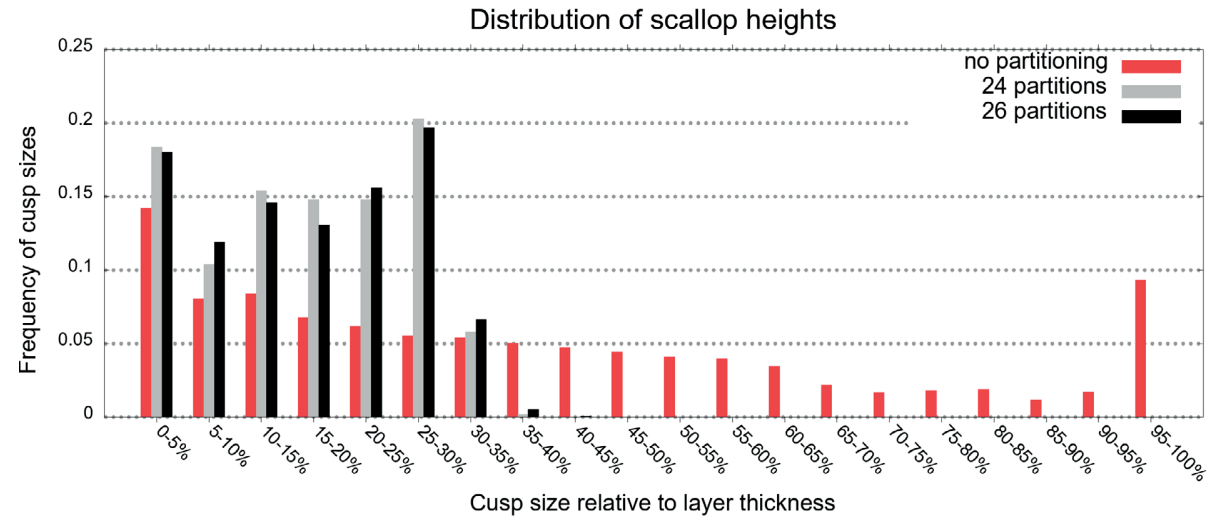

Figure 96: Distribution of cusp sizes for the part consisting of 32 partitions

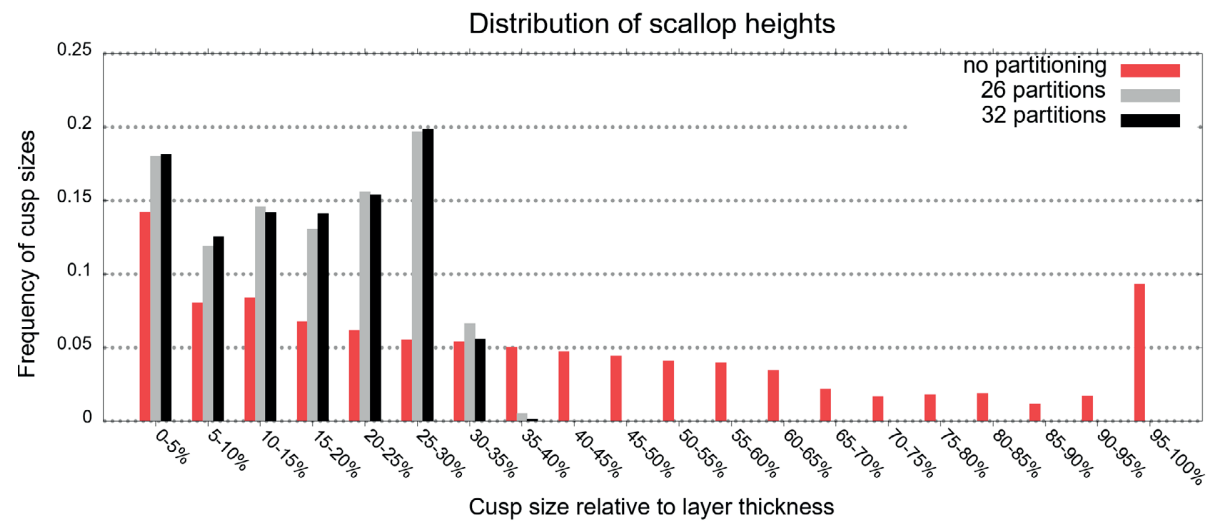


Figure 97: Average cusp height for different number of partitions, note that for no partition (classical 3d printing without $3+2$ ) the average cusp height is 0.377

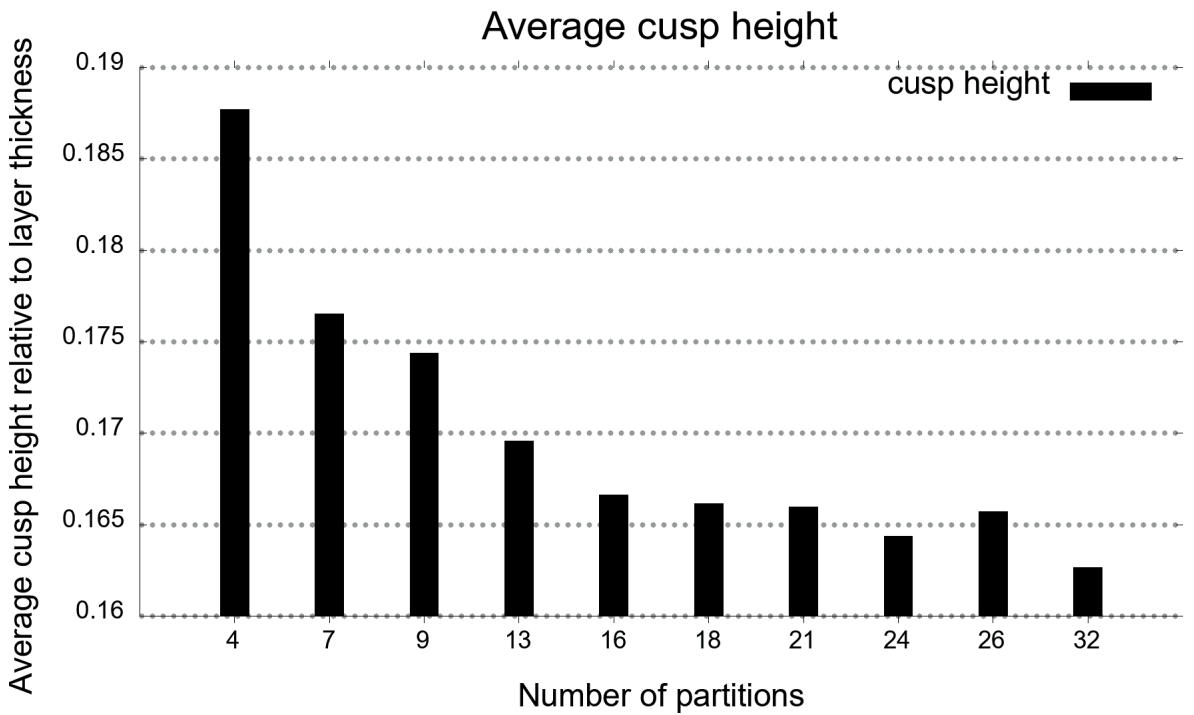

\subsection{GREEDY VOLUME SPLIT/DECOMPOSITION BASED ON HALF-SPACES}

In addition to the algorithm developed in section 3.2, a variation of the half-space partition based on a greedy approach has also been developed. The greedy algorithm performs the decomposition plane search differently and does not use an error function explicitly. The greedy algorithm starts by scanning the part surface with a plane perpendicular to the $Z$ direction, moving outward from the table. Throughout this process, the plane orientation remains constant while the plane moves upward and terminates at the first encountered overhang or another criterion. Then, the algorithm defines a box around the overhang region to search for the split plane. The scanning plane may adjust its orientation to search for its optimal position while analyzing the surface geometry in the vicinity of the intersection between the scanning plane and the part surface.

Instead of more closely defining the details of this complex problem-constraints due to accessibility, collisions and the inevitable need for support structures in some cases-it can be defined as a limited problem as follows.

There is a given free-form geometry. This geometry is guaranteed to be manufacturable under the mentioned constraints. If not, the algorithm should detect any infeasible shapes. For the given free-form geometry located on a table, scan the part surface parallel to the table with plane $E$, starting with the scanning plane $E_{0}$ intersecting point $\boldsymbol{P}_{\mathbf{0}}$ and iterating forward in the direction of the table normal until it reaches point $\boldsymbol{P}_{\boldsymbol{i}}$, in which $E_{i}$ encounters deposition 
conditions that must be avoided, like the overhang on the right side, as shown in Figure 98 and Figure 99. Then, a proper splitting plane (last feasible $Z$ scanning plane) must be found. Since an infinite number of planes can be constructed, the goal is to search (shift and rotate) for a plane position that meets certain criteria. Different objectives may result in different planes, like planes $E_{i}^{\prime}$ and $E_{i}^{\prime \prime}$, as shown in Figure 98. The sub-volume between the table and the next splitting plane is the result of the greedy decomposition, and it is considered for further slicing and toolpath planning. The sub-volume is also removed from the part geometry, and the next iteration begins scanning the remaining part geometry from the splitting plane as if it were a table.

Figure 98: Sweep-plane scanning of the part geometry

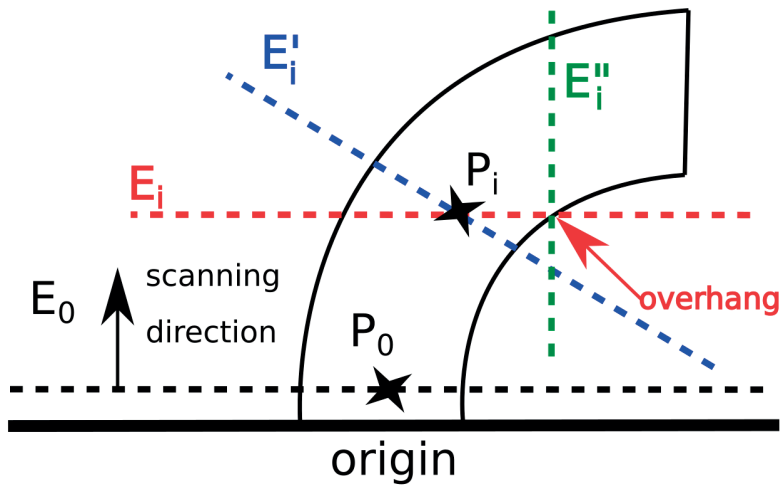

Figure 99: Slicing until overhang of 45 degrees (red)

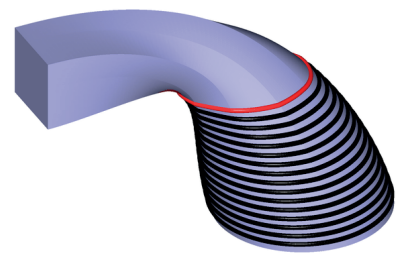

a) side view

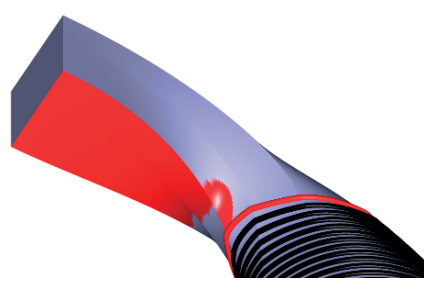

b) bottom view 


\subsubsection{Construction of the search space}

Parallel scanning begins from the table then incrementally progresses upward. Parallel scanning terminates as soon as either the staircase measure or overhang threshold angle $\alpha_{\text {threshold }}$ is exceed. Following that, the splitting plane is to be found. In order to improve the computational burden, the search for the splitting plane can be restricted to a smaller volume. The lower bound is the last feasible scanning plane $E_{i-1}$. Another boundary can be the utmost plane $E_{i}$ " defining the overhang threshold for the current printing direction, as shown in Figure 100. The goal is to iterate through the search points scattered on the part surface within the axis-aligned bounding box (AABB) so that all search points below the optimal splitting plane satisfy a function $F$, defined as the number of search points below a plane $E$. All search points below $E$ must tolerate upskin/downskin thresholds, otherwise $F$ becomes invalid.

Figure 100: Bounding box of the search space

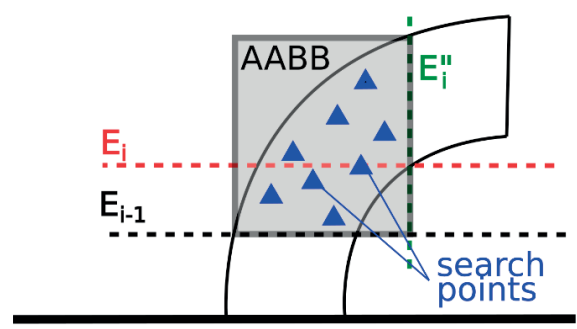

a) $2 d$ representation

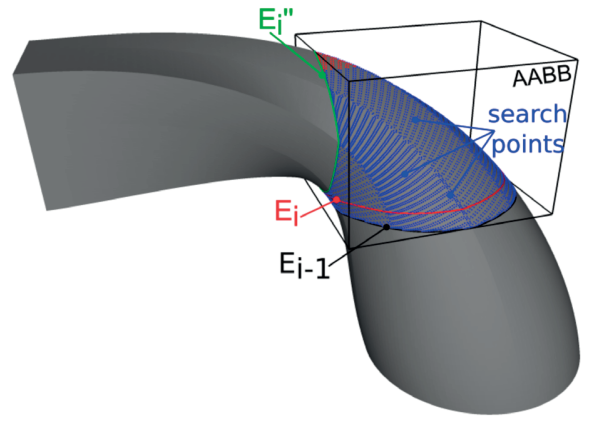

b) $3 D$ representation

The search space is intentionally restricted to a prismatic primitive (AABB), although ideally, the search space should be a truncated pyramid with the base comprised of the part profile polygon at the utmost horizontal scanning plane and the face slope contributing to the maximal allowed overhang, as depicted in Figure 101a. On the one hand, the use of AABBs limits the variety of geometries that can be handled by the algorithm. However, on another hand, splitting planes may not have a negative slope, thus collision-free process planning becomes easier.

Search points are sampled on the part surfaces with any given precision. Successful search points must satisfy the specified quality and threshold criteria (mesh orientation at search points must tolerate upskin/downskin thresholds). Not all points within the AABB are considered for the search. The upskin and overhanging points are excluded from the search, as shown in Figure 101b, because any search plane at these points will generate insufficient surface quality. 
Figure 101: Search points within AABB

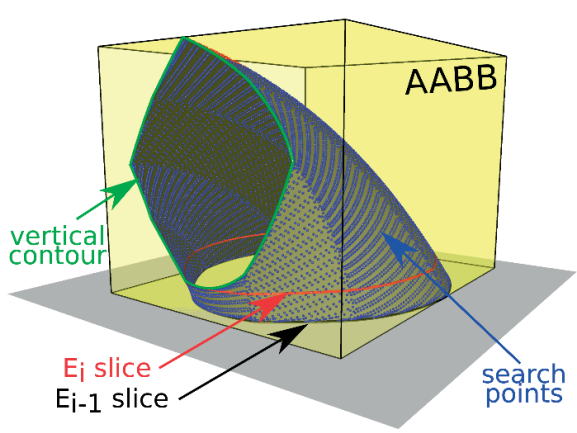

a) Boundary curves of $A A B B$

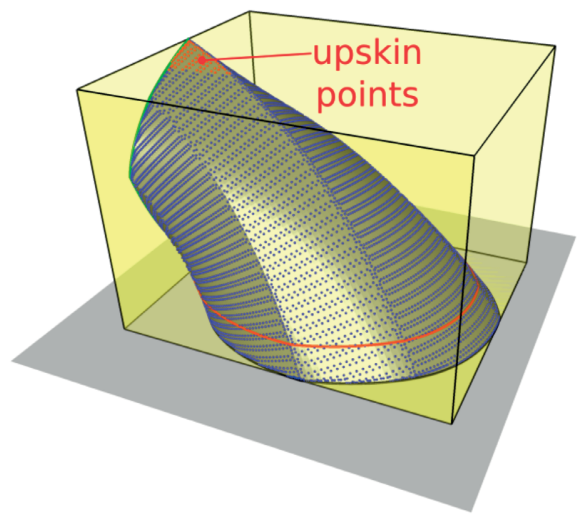

b) Upskin points excluded from search

We use search points $\boldsymbol{P}_{\boldsymbol{i}}$, where each $\boldsymbol{P}_{\boldsymbol{i}}$ has an according normal $\overrightarrow{\boldsymbol{n}_{\boldsymbol{\imath}}}$, which is still the triangle normal coming from $\overrightarrow{\boldsymbol{n}_{\boldsymbol{\imath}}}$ of triangle, where $\boldsymbol{P}_{\boldsymbol{i}}$ is derived from. Accordingly, each search point, in addition to its spatial position, must be associated with a Boolean value. This approximation simplifies the calculation of the function $\boldsymbol{F}$.

\subsubsection{Search of the splitting plane}

The optimal splitting plane position is sought at search points via brute-force computation, with fixed sampling of angles $\varphi$ and $\theta$ defining the plane. For each search plane, the number of points below the plane are counted in order to select the plane with the greatest number of accepted points, as shown in Figure 102a. However, not all search planes are feasible. If a splitting plane intersects cross-section contours on the faces of AABB (vertical contour and $E_{i-1}$ slice contour in Figure 101a), the connection between two sub-volumes will be represented by two different planes, thus making parallel slicing for the next sub-volume impossible. Figure $102 \mathrm{~b}$ shows a plane intersecting the bottom contour of the AABB. This is therefore categorized as a non-feasible search plane.

For this AABB, there are 5059 search points along with 9280 triangles to be checked at the utmost $90^{\star} 360=32,400$ plane orientations. 
Figure 102 Search plane test

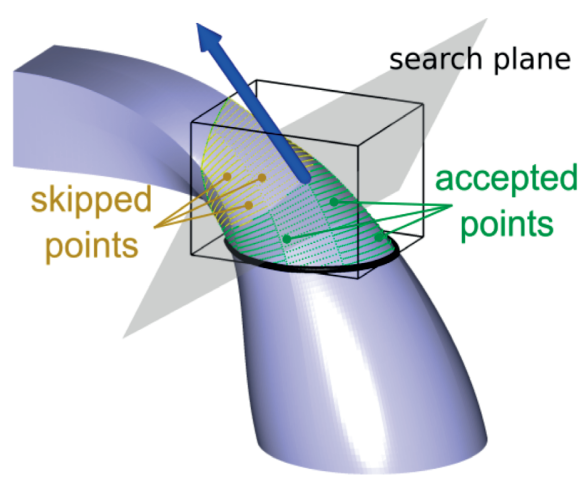

a) Point counting

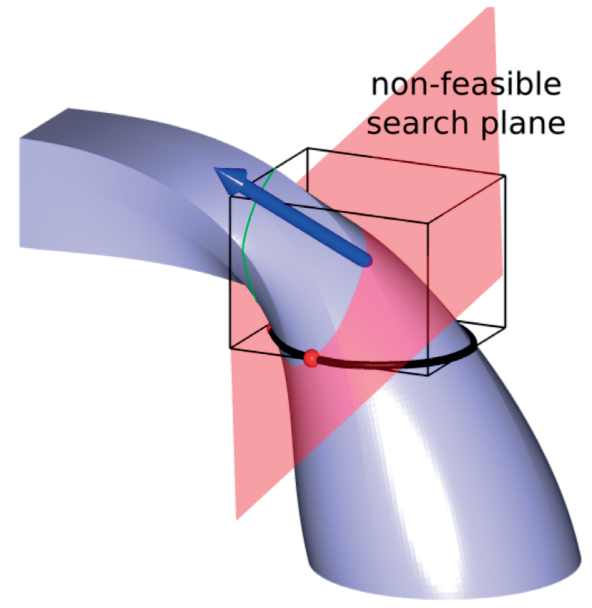

b) Intersection of the bottom contour

Eventually, the greedy algorithm can be used by two algorithms to find a decomposition plane and counting of the valid points, as defined in the pseudo-code in Figure 103. 
Figure 103: Pseudo-code of the greedy algorithm

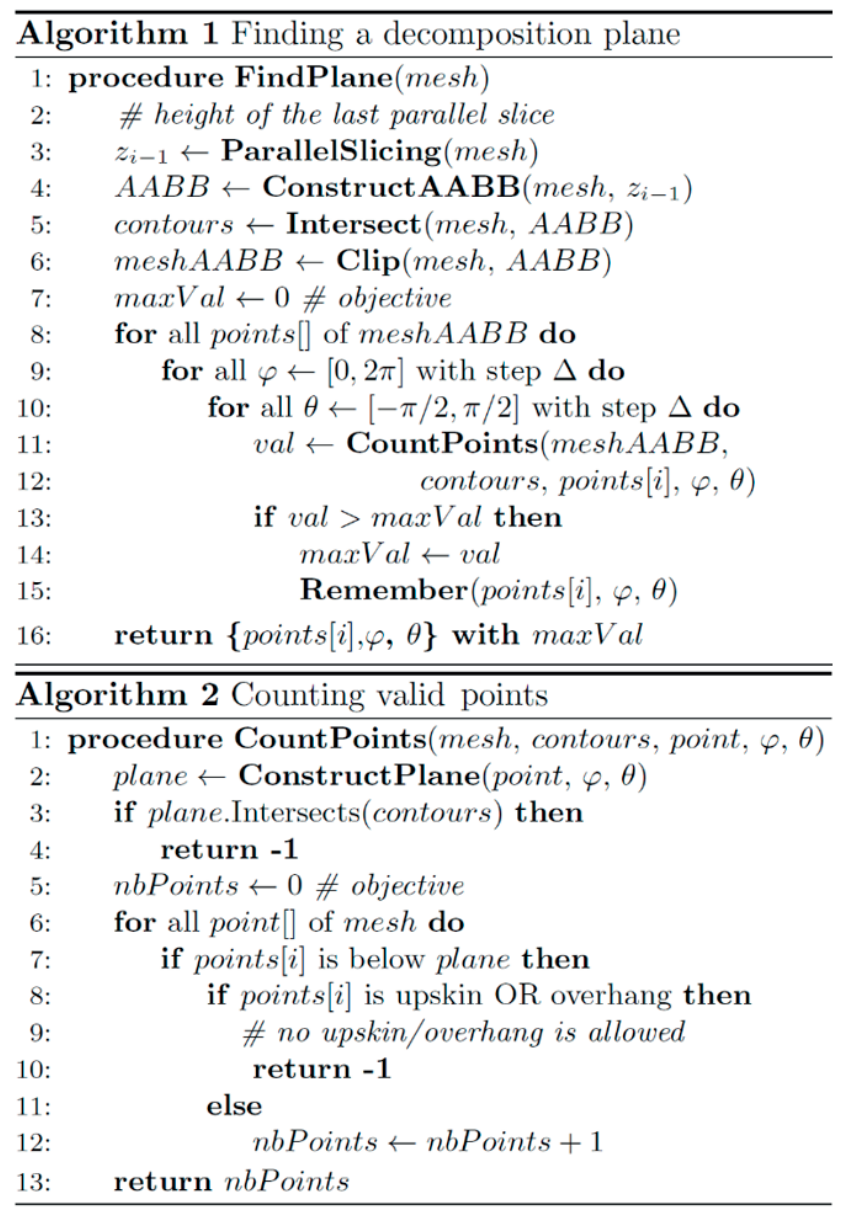

\subsubsection{Applying different conditions}

Different boundary conditions may be applied on the search criteria. Figure 104 shows three different splitting planes. Two planes are found with restricted plane orientation (45 degrees and 90 degrees). The third result included an additional condition not to include upskin points with surface orientation exceeding 60 degrees. 
Figure 104: Results of the greedy algorithm

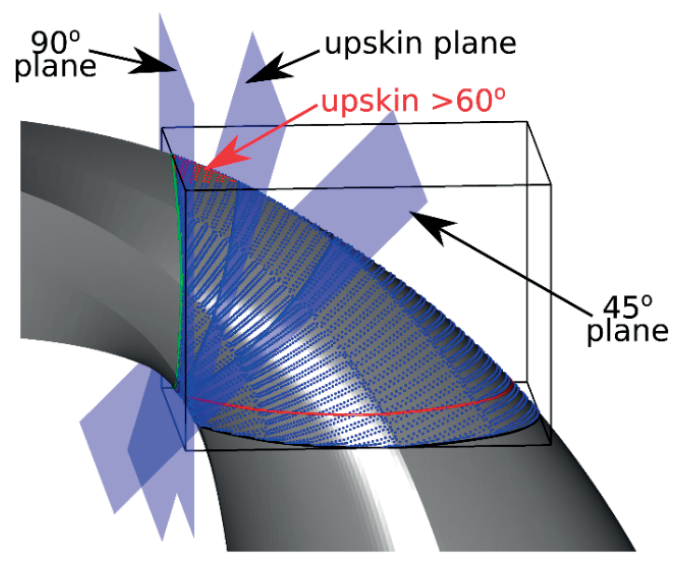

Different weights for the overhang and gravity converge to different decompositions and require different process planning, as shown in Figure 105, Figure 106, and Figure 107.

Figure 105: Slicing for the greedy algorithm strategy with the fixed 45-degree splitting plane

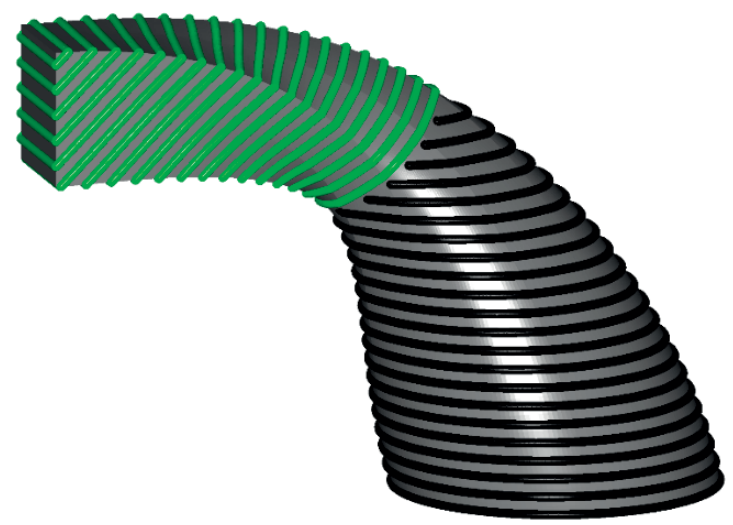


- Figure 106: Slicing for the greedy algorithm strategy excluding the 60-degree upskin

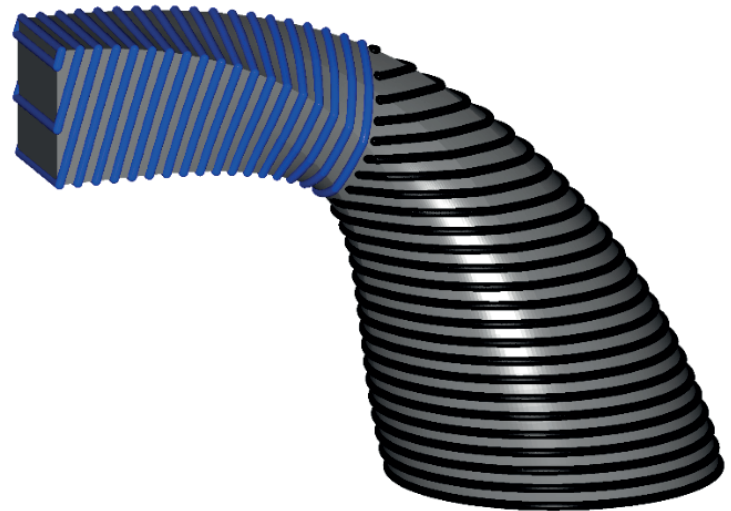

Figure 107: Slicing for greedy algorithm strategy with a fixed 90-degree splitting plane

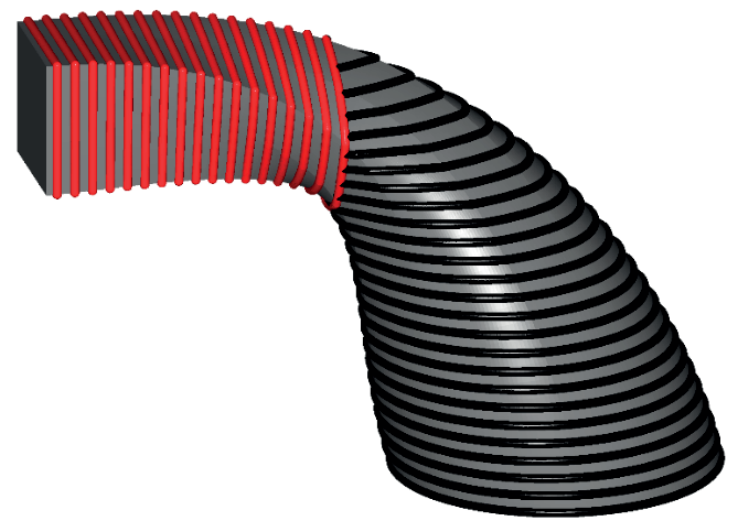

Figure 108, Figure 109, and Figure 110 show how the distribution of scallop heights looks for each of the three examples. The surface quality has improved by reducing high-magnitude heights. However, this is a coincidence for this particular shape. No one should expect quality improvement beyond the defined rules (avoiding upskin, etc.). The average cusp heights are $0.362,0.382$, and 0.267 of layer thickness for the 45 -degree, 60 -degree upskin, and 90 -degree strategies, respectively. 
Figure 108: Distribution of cusp sizes for the greedy decomposition with a 45-degree limit

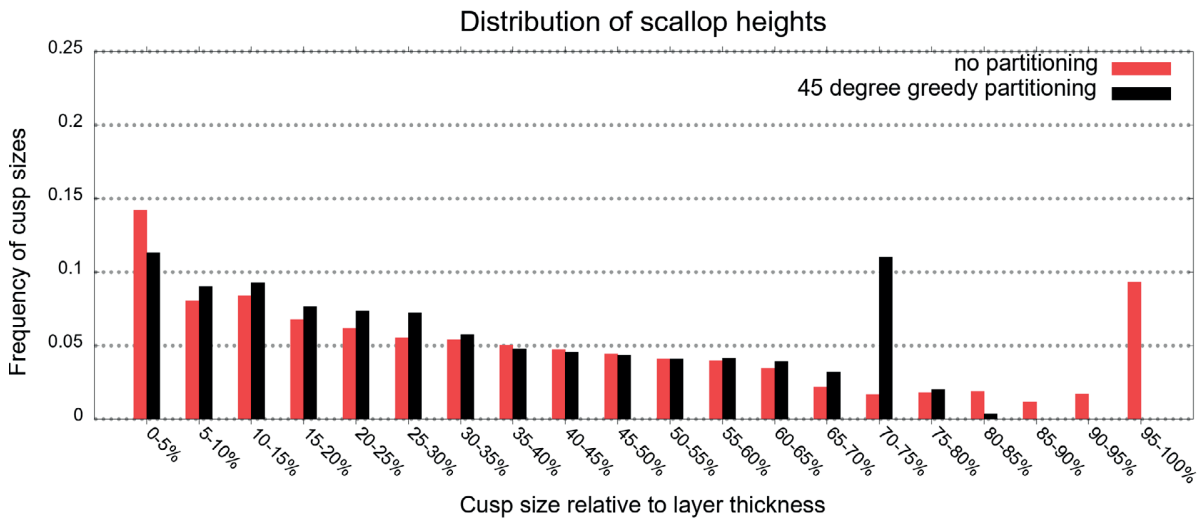

Figure 109: Distribution of cusp sizes for the greedy decomposition excluding the 60-degree upskin

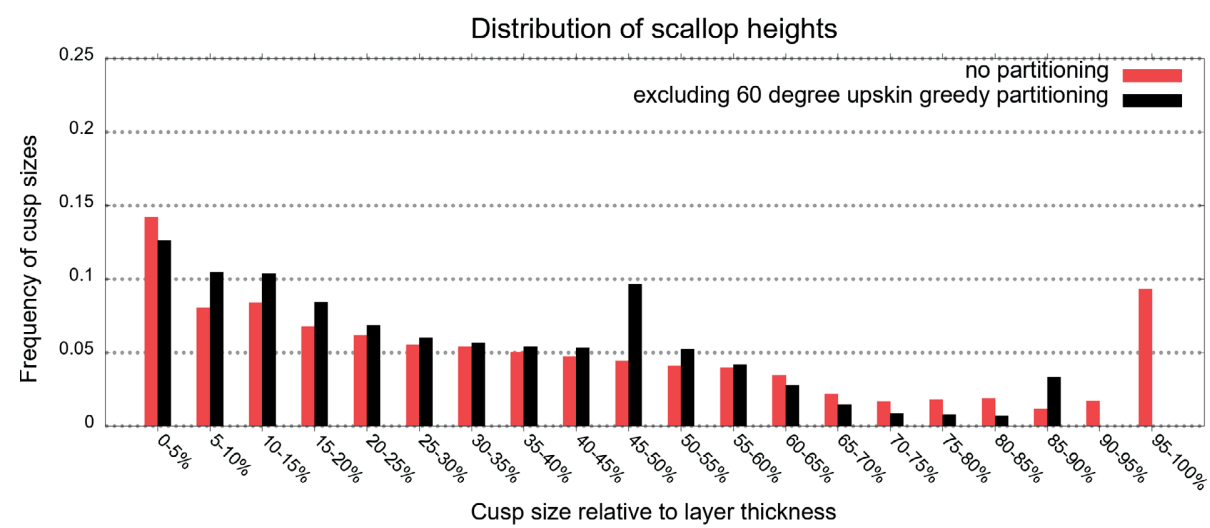

Figure 110: Distribution of cusp sizes for the greedy decomposition with a 90-degree limit

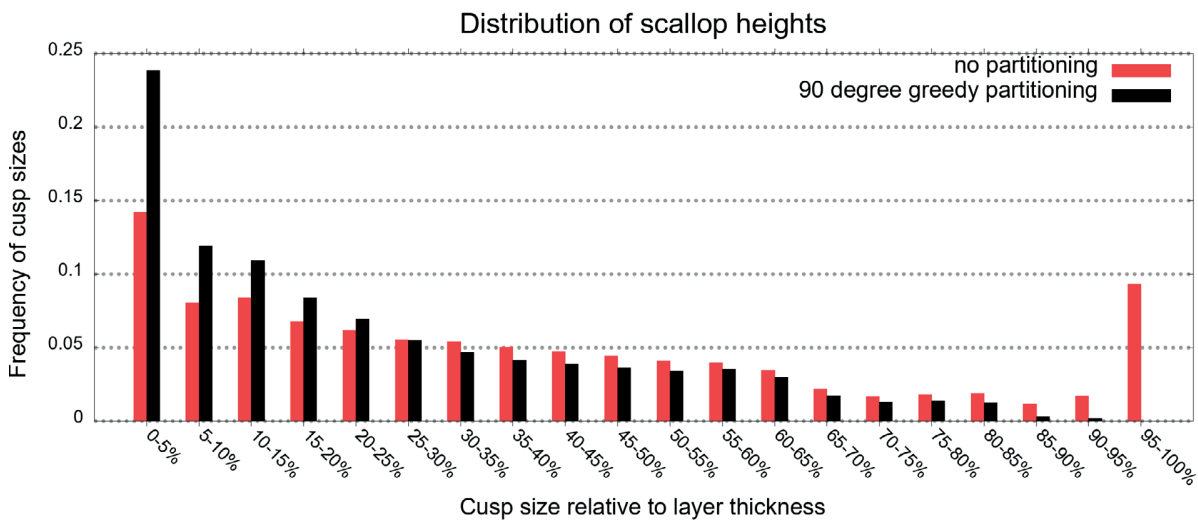


Comparison of average cusp heights of the general brute force algorithm and the greedy algorithm show that both algorithms result with a smaller cusp height compared to 3 axis printing. For the given test part, the greedy algorithm is generating two sections, which result between 0.267 and 0.362 average cusp height depending on the 45 to 90 degrees upskin limit. The brute force algorithm provides a resulting between 0.16 and 0.19 range average cusp height based on the number of generated sections which are between 32 and 4. Note, the average cusp height values mentioned here are all based on layer height.

Although the brute force algorithm delivers better average cusp values than the greedy algorithm, the greedy algorithm has a performance advantage due to less computationally intensive finding of split plane. The brute force algorithm requires three nested loops to find the split plane's orientation and position in space and then go over all triangles to calculate the error function for each case.

\subsection{PRINTING EXPERIMENTS USING THE DEVELOPED ALGORITHM}

The focus of the sections 3.1 and 3.2 has been the development of an algorithm for $3+2$ axis $\mathrm{AM}$ and the validation of the algorithm using computer simulation.

After final implementation and validation of the algorithm using computer simulation, Frikel (Frikel, 2020) applied the general brute force algorithm from section 3.1 and 3.2 to a newly developed five-axis printer VSHAPER from the company Verashape in Poland and documented the results of the experiment. It has to be noted that the experiment described below does not aim to validate the developed algorithm in terms of the cusp size. Rather the experiment is aimed to observe and document issues arising by multi axis FDM on a newly developed 5 axis FDM printer using the above developed algorithm and its software implementation as a tool. Further, the algorithm has been modified for printing the $Y$ shape illustrated in Figure 118 since the $Y$ shape requires branching of solutions, which is not supported with the base algorithm which is designed to work sequentially.

Deposition of the initial test part, a pyramid shape, is illustrated in Figure 111, where it was decided to demonstrate how the segmentation method can be implemented. The process parameters that were used to build the part are summarized in Table 2. PLA with a diameter of $1.75 \mathrm{~mm}$ was used as the filament for the build-up. The nozzle diameter was $0.8 \mathrm{~mm}$, so the optimal layer height for the initial layer but also for the other layers was $0.4 \mathrm{~mm}$. Based on the nozzle diameter, a path width of $0.8 \mathrm{~mm}$ resulted. Triangles were used as a pattern for the filling. The initial layers, as well as the top layers, had simple lines as a pattern with an angle change of 45 degrees between the layers. As filling for the part, a degree of 20 percent density was chosen (Frikel, 2020). 
Table 2: Main process parameters used for the trials (Frikel, 2020)

\begin{tabular}{ll} 
Process parameter & Value \\
\hline Initial layer height & $0.4 \mathrm{~mm}$ \\
\hline Layer height & $0.4 \mathrm{~mm}$ \\
\hline Path width for inner/outer walls and infill & $0.8 \mathrm{~mm}$ \\
\hline Number of border layers (walls) & 3 \\
\hline Number of top layers & 3 \\
\hline Number of bottom layers & 3 \\
\hline Travel speed & $900 \mathrm{~mm} / \mathrm{min}$ \\
\hline Nozzle diameter & $0.8 \mathrm{~mm}$ \\
\hline Density infill & $20 \%$ \\
\hline Filament diameter & $1.75 \mathrm{~mm}$
\end{tabular}

In general, the segmentation approach has worked well, as shown in Figure 112 for the two different areas of the part. After applying some layers, measurements with a 3D microscope were performed to check if the achieved layer height was at the same level as expected. The results showed that the desired height was reached (Frikel, 2020).

Figure 111: Pyramid-like part (Frikel, 2020)

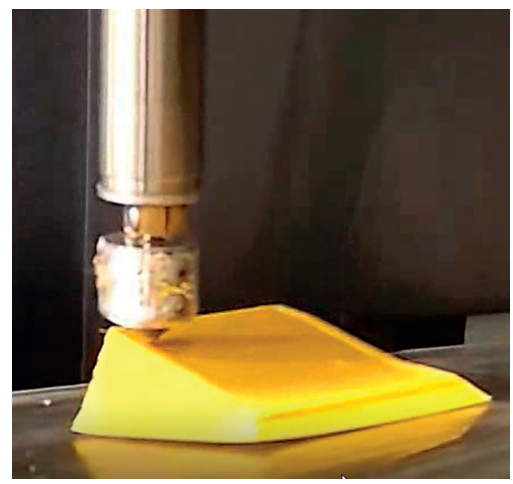

Figure 112: Layer height measurement with 3D microscope (Frikel, 2020)
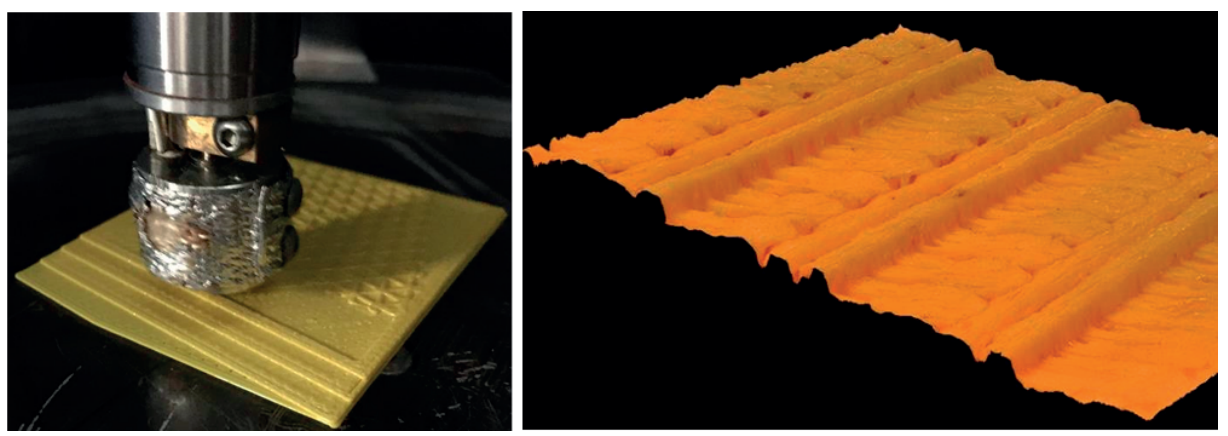
Microscopic pictures of the infill pattern in the upper region also showed expected results as shown in Figure 113 (Frikel, 2020).

- Figure 113: 3D microscope image of infill pattern in upper regions (Frikel, 2020)

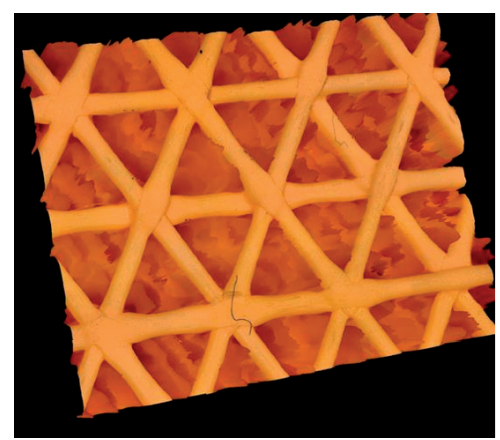

The part could be built, but it still had minor defects. Addressing these deficiencies would lead to better print quality. In the following section, the deficiencies are explained and steps to counteract them are listed (Frikel, 2020).

Figure 114 shows the first deficiency observed when building up the part. At the beginning of every single segment, there was too much material applied. Each individual layer was then examined in the toolpath and no irregularities, such as paths outside the part geometry or overlapping of paths, were found. Thus, the deficiency must be due to the process parameters. Process parameters are mostly handled in the post-processor. With the help of reports in the toolpath, it is possible to detect whether the area applied is a wall, an infill or a link move. With this information, the deposition rate for the wall of every first layer of a segment can be adjusted in the post-processor for the NC output (Frikel, 2020).

Figure 114: Beginning of segments with too much material (Frikel, 2020)

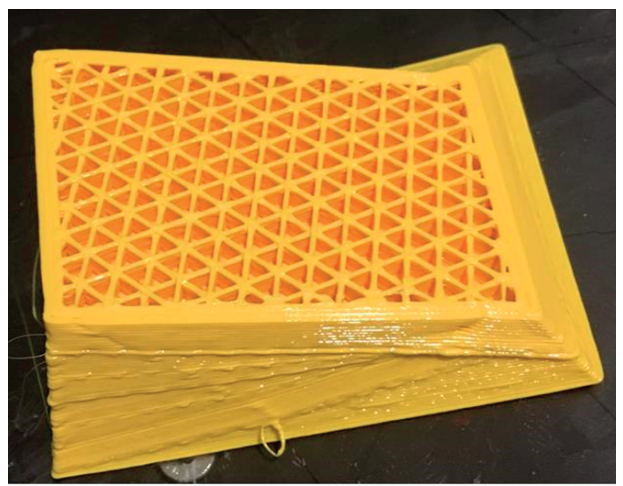


Figure 115: Measurement of wall thickness (Frikel, 2020)

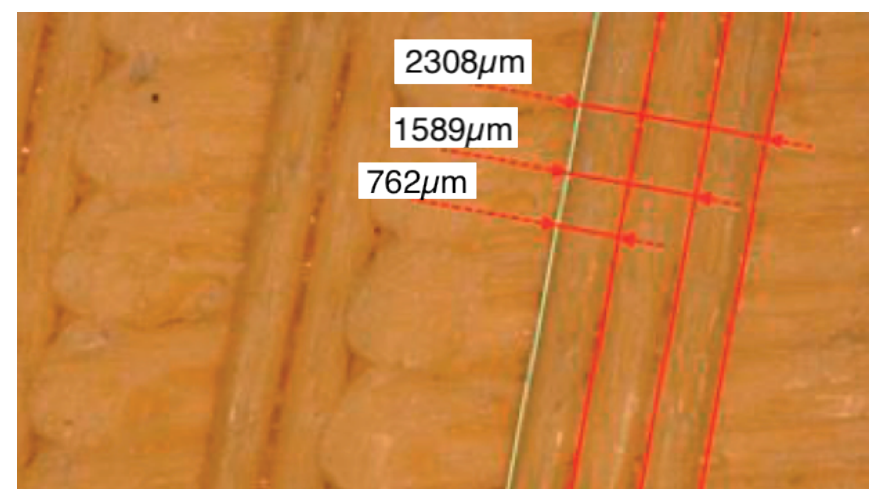

When measuring the wall thickness (Figure 115), it was detected that some infill paths were overlapping the walls. In this respect, an examination of the toolpath also showed no abnormalities. No overlap could be observed during the toolpath generation. As shown in Figure 115 , it also only happened for three infill lines and not for all the others. This abnormality must be further observed, and whether or not it is not due to mechanical problems must be established. If it is observed continuously, there is a parameter that can be used to define the level of overlap between the walls and infill (Frikel, 2020).

The last identified deficiency is illustrated in Figure 116. Printing of the first layers was performed at a travel speed of $1200 \mathrm{~mm} / \mathrm{min}$. Therefore, the material had less time to cool down and a good adhesion of the first layers to the building platform was not achieved. As a result, the part lifted from the building platform. This problem can be solved in three different ways. One possibility would be to add a so-called brim. Brim means adding additional material around the part for the initial layers to ensure the adhesion. It could also be additional layers printed with an area larger than the actual part. The build-up of the real part could then be performed on top of these additional layers. This would also guarantee good bonding. Another solution to the problem would be to build the initial layers at a lower travel speed. As a result, the material would have more time to cool down and adhere strongly to the building platform. As a last option, additional waiting time could be added to ensure there is sufficient cooling time. For this purpose, the print time for each individual layer is already calculated during the toolpath generation. Depending on how long the printing time of each layer is, dwell time commands can be written into the NC code. A sample of such an output is illustrated in Figure 117. It was specified that if the layer was printed in less than 10 seconds, then the machine should wait for five seconds before continuing the printing process. Both the threshold for the printing time and the wait time can be defined in the post-processor (Frikel, 2020). 
Figure 116: Part lifting from the building platform (Frikel, 2020)

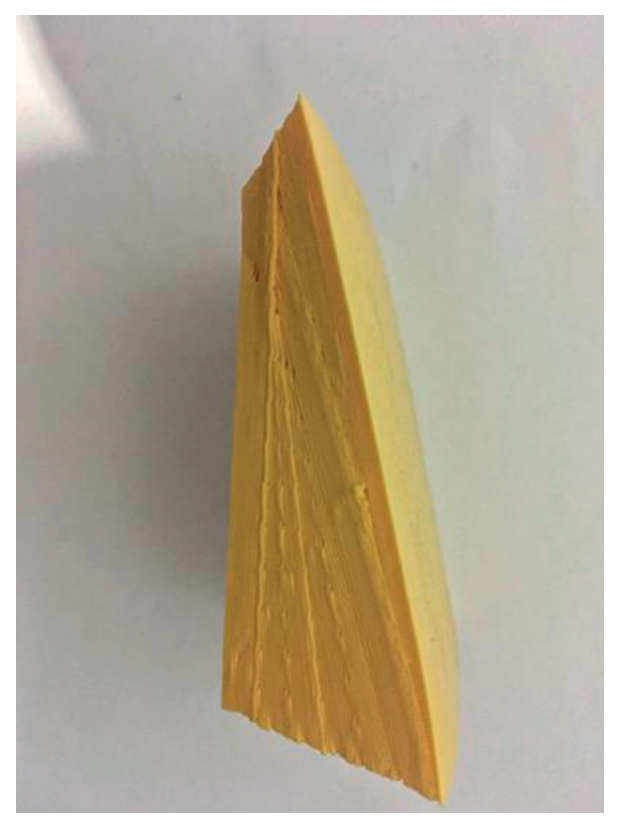

[ Figure 117: Dwell time command output depending on print time (Frikel, 2020)

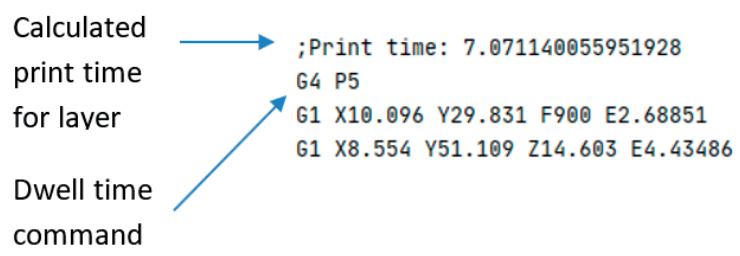

The second part that was machined with the help of VSHAPER is illustrated in Figure 118. This $\mathrm{y}$-shaped tube with a wall thickness of $4 \mathrm{~mm}$ was built by VSHAPER to test the capability of the segmentation strategy. The process parameters and the material used were the same as those used for the first part. All in all, this component could be built with this approach, but like the previous part, it had some minor defects (Frikel, 2020). 
Figure 118: Thin-walled y-shaped tube (Frikel, 2020)

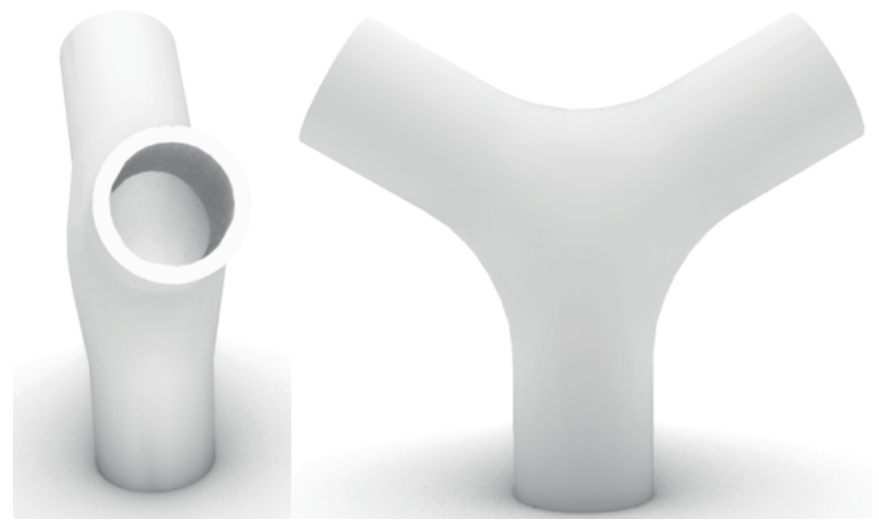

Due to the fact that the component's walls are so thin, it quickly became apparent that the patterns currently available for filling should be extended. In order to be able to print this type of component faster, and to achieve better mechanical properties, a pattern like the one shown in Figure 119 is a good choice. Each layer could be applied by a continuous path using this kind of pattern (Frikel, 2020).

Figure 119: New possible pattern type for infill (Frikel, 2020)

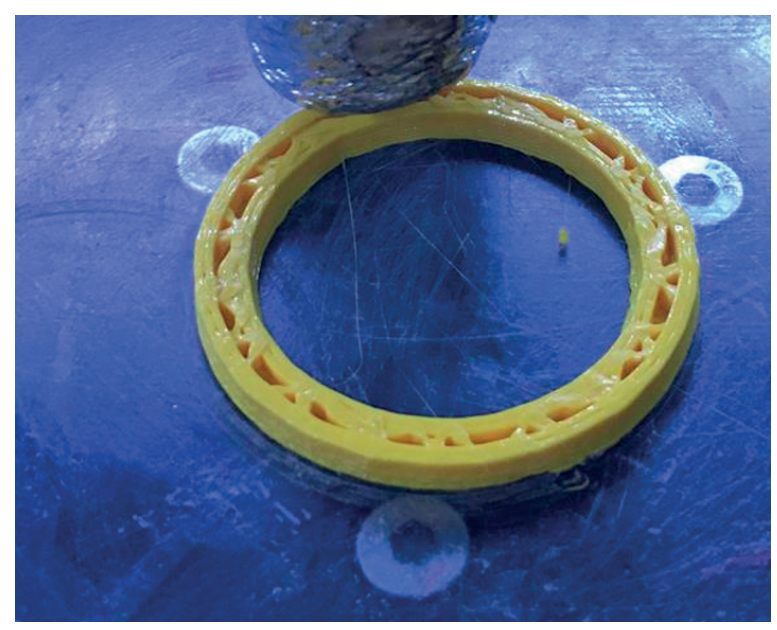

In areas where the segments are small, there is not enough time for the material to cool down, so the bonding between the segments is not sufficient. The material deposited is not completely solid before the next material is applied. Due to gravity, the material flows down a little and gaps between the layers occur. This can be solved by adding some dwell time after printing 
small areas. It is the same approach as introduced for the previous part. Moving slower when building up small areas also allows more cool-down time (Frikel, 2020).

[ Figure 120: Insufficient bonding when printing small areas (Frikel, 2020)
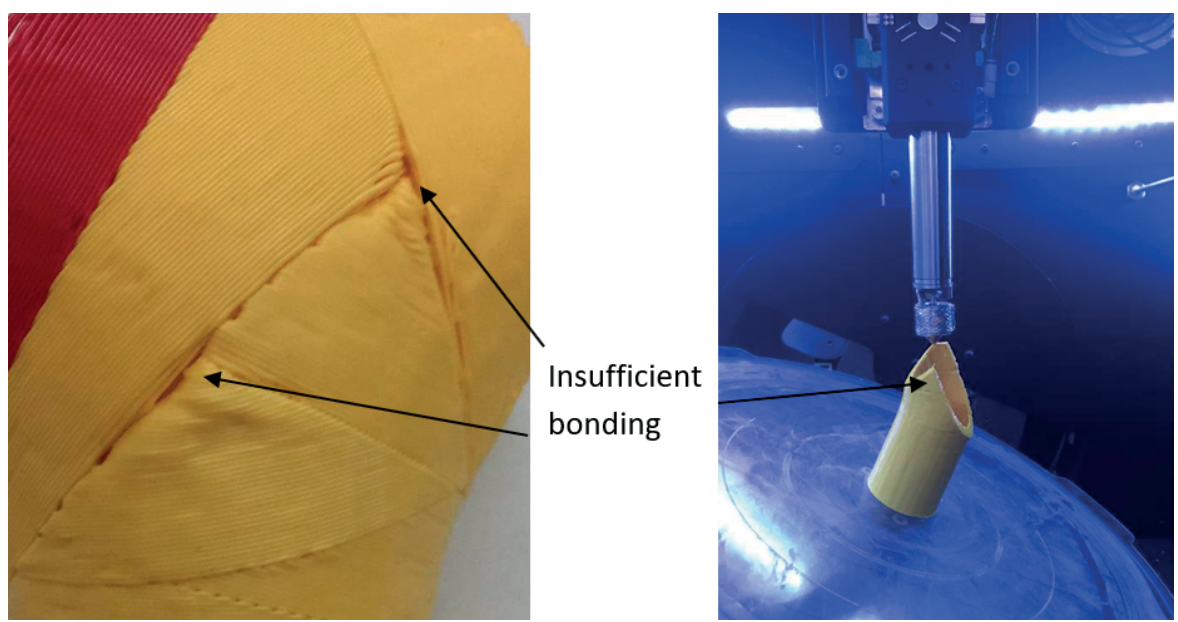

The result of adding dwell time after printing small areas and using a lower travel speed can be seen in Figure 121. Please note that, as highlighted in light blue, the gap has become noticeably smaller. However, small gaps still occur. Those gaps can be closed by moving over the area just with the hot extruder, without applying any material or just a small amount of material. This process is called ironing and can also be activated in the additive module. For the first test, it was not used. It needs to be investigated whether the parameter corrects this deficiency or whether further adjustments of this parameter are necessary (Frikel, 2020). 
Figure 121: Result with more cool-down time (Frikel, 2020)

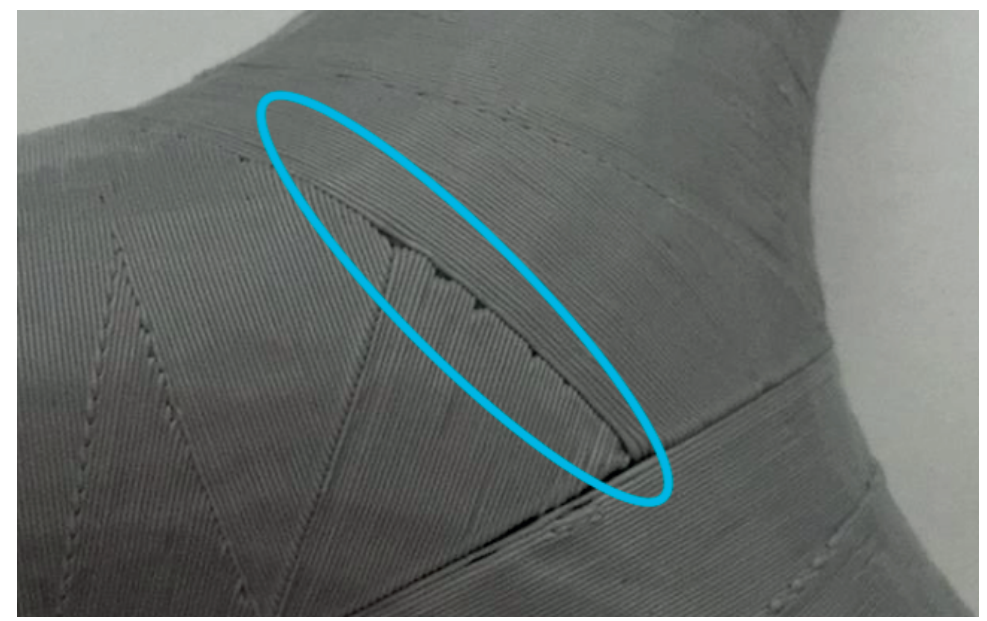

During the build-up, the link moves between the single layers that were applied on the inside of the part. This led to so-called "stringing" on the inside of the pipe, as shown in Figure 122. Link movements are inevitable. To avoid this stringing inside the pipe, it would be better to perform these movements along the contour of the pipe. It would apply additional material, but this is minimal. If this is also to be avoided, then link movements could also take place outside the part. It is easier to post-process any stringing on the outside of the piper rather than on the inside. Another possibility would be to minimally retract the print nozzle before the link movement. This strategy is called " $z$-hop" and is also already available in the additive module. This prevents the nozzles from minimally dragging the already applied material away from the layer that has just been applied (Frikel, 2020).

Figure 122: Stringing (Frikel, 2020)

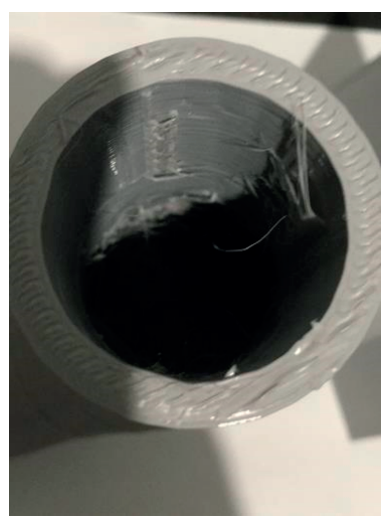


In the first test, this pipe was printed without brim or adding additional layers before printing the part. Due to its thin walls, the pipe did not adhere well to the building platform. This issue was solved by adding three $100 \times 100 \mathrm{~mm}$ layers with a layer height of $0.4 \mathrm{~mm}$ beforehand. The result is shown in Figure 123. The pipe has bonded well to the additional layers and did not delaminate (Frikel, 2020).

The experiment described above used the implemented $3+2$ algorithm for a variety of parts. The conclusion of this experiment is that many issues related to multi-axis FDM printing has been identified, that have not been the focus of the $3+2$ algorithm. Further research is needed on these identified topics.

Figure 123: Additional layers for better adhesion (Frikel, 2020)

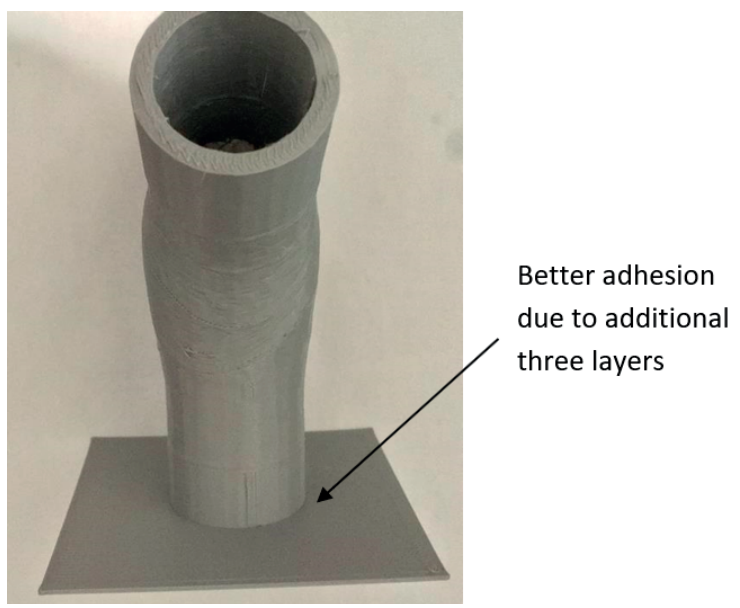

\subsection{CONCLUSIONS}

In this chapter, a novel approach for $3+2$ axis AM is presented for FDM and DED. The definition of a $3+2$ axis process is that after applying 2.5D slicing-based deposition, the direction of slicing is changed so that many partitions, each with their respective slicing directions, are required. Commercial 3D printers have only three degrees of freedom, so the $3+2$ axis AM process requires hardware that allows for a change of deposition direction.

The novel approach for $3+2$ axis AM has been implemented using a global error function. For comparison purposes, a simple greedy approach is also presented. Both algorithms are implemented as a software program so they can be tested using sample parts for validation. The resulting partitioning of the parts showed that, for both algorithms, the results were calculated in line with the design purpose of the algorithms. 
For validation purposes, the surface quality measured in cusp height has been defined as a metric using a distribution scheme, and the improvement in both the distribution of errors and average error values are compared for 3D printing without $3+2$ axis $A M$ and for the different number of partitions using the $3+2$ axis $A M$ algorithms that were developed here.

The validation resulted in a significant improvement of surface quality. For the tested part, the quality improvements were much greater as the number of partitions increased and reached saturation at some point. Measured in average cusp height relative to layer thickness, the classical $3 \mathrm{~d}$ printing delivers the value 0.377 , whereas $3+2$ axis AM with 4 partitions lower this value to 0.187 . With 32 partitions, the value of 0.163 could be reached.

The resulting software has been made available for further research, and as part of a Master's thesis, the software has been applied to a physical printing experiment for a newly developed five-axis 3D printer VSHAPER by the company Verashape from Poland. The results of this Master's thesis are also included in this chapter (Frikel, 2020). It showed that, the algorithm was able to calculate $3+2$ axis $A M$ tool paths for all test parts presented in the physical printing experiment.

The algorithm based on global explicit error functions is designed to process non-branching geometries. Decomposition of branched structures is under development by extension of the existing algorithm, and the initial results are presented in the outlook in section 5.2.

As a further outlook, the algorithm can be improved by adopting adaptive slicing (layer thickness) to reduce the stair-stepping effect on a curved surface. The missing functionality may be extended by including a collision check and avoidance of sub-volume decomposition and slicing, as well as implementation of non-uniform and curved slicing.

As a final conclusion, it will be checked if the research questions from Chapter 1 have been addressed. What are the answers to these questions?

Research question: Can novel multi-axis AM toolpath planning strategies be developed that result in reduced or no support structures?

This chapter presents a novel multi-axis AM toolpath planning strategy that overcomes the limitations of the state-of-art approaches in this area by presenting a geometrical algorithm based on a global error function to decompose the input shape into sub-volumes with their respective deposition orientations while preserving the 2.5D layer-by-layer deposition concept of a traditional fixed-orientation deposition. The validation using simulation has proven that the need for support structures has been eliminated for the test cases. Such the research question can be answered positively. 
Research question: Can novel multi-axis AM toolpath planning strategies be developed that result in improved surface quality through minimization of the stair-stepping effect?

The algorithm presented in this chapter, which is based on a global error function, uses the target criteria of reducing the stair-stepping effect. It tries to decompose the input shape into sub-volumes with their respective deposition orientations to minimize the stair-stepping effect while respecting other constraints. The validation was achieved using a simulation, which proved that the stair-stepping effect can be drastically reduced compared to traditional 3D printing in the test cases. Accordingly, this research question can be answered positively as well.

Research question: To what extent modifications of existing subtractive path planning strategies and related algorithms can be used for multi-axis AM?

The algorithm presented in this chapter is based on the geometric decomposition of an input shape using a global error function. In five-axis milling, the aim is to subtract volume which is selected by user so that the geometric models and algorithmic approaches are not comparable. 

CHAPTER 4

I True Multi-Axis AM Toolpaths 
True multi-axis AM is ideally suited to the accurate repair of used parts (e.g., dies and molds) or fabrication from scratch of complex geometry products (near net shape production for the aerospace, mining, energy and medical industries). The process planning for repair applications is more challenging in comparison to fabrication from scratch because the deposition is restricted to a given volume, while the geometry of the existing part must also be considered.

The process planning for both fabrication from scratch and repair applications requires a defined volume that must be filled with material. Usually, the volume is defined as a CAD model. In repair applications, the volume defines the missing material that was worn or broken during the exploitation. In this case, the volume is computed as a difference between the target shape and the actual shape obtained with measurements. Figure 124 shows how the steps of the repair may look. It starts with measuring the actual workpiece. Then, the toolpath generation must ensure that the required volume is filled with paths. Additionally, due to the presence of obstacles, it must be ensured that all geometries (nozzle, workpiece, deposited material, fixtures etc.) will not collide. Due to the complexity of the process utilizing multi-axis machines or robots for deposition and applying complex path planning by process engineers, the toolpath must be optimized, simulated and verified. Eventually, the material is deposited and inspection (QA) is applied.

Figure 124: Steps of the repair operations
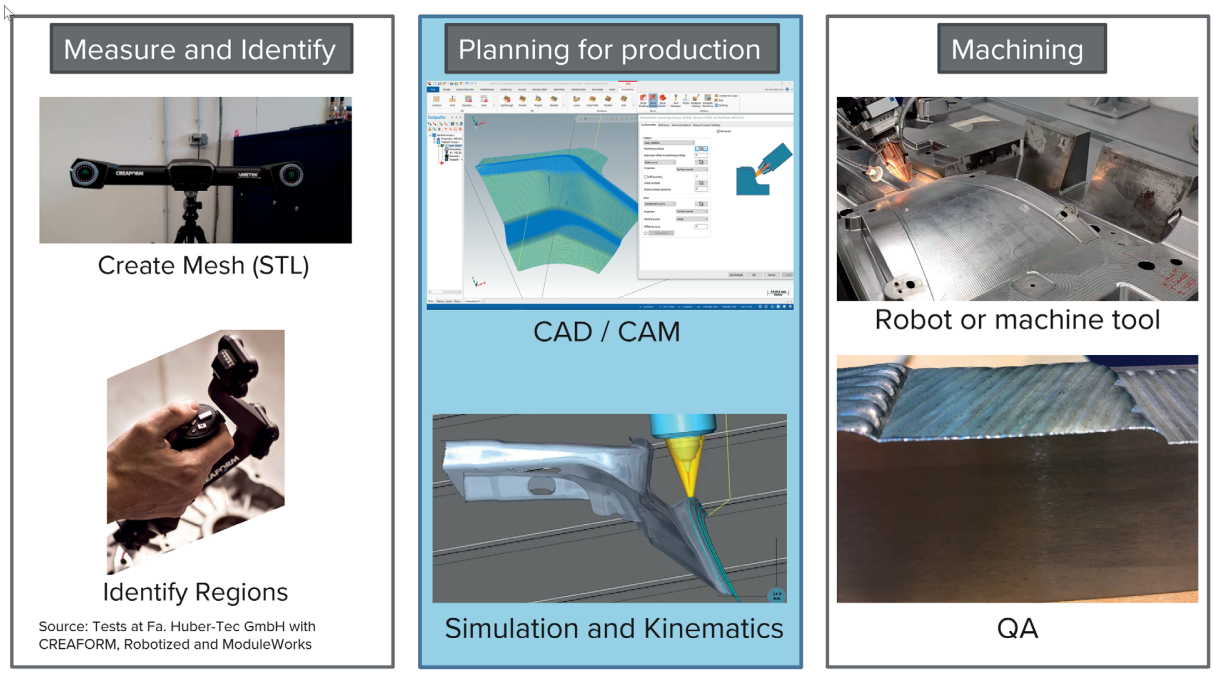
As has already been highlighted, CAM planning includes several steps. The path planning for the additive steps of the repair consists of three major steps:

\section{Generation of curved slices to fill a given volume}

2. Generation of equidistant toolpaths at every curved slice

3. Planning of the nozzle orientations to prevent collisions

The following sections present algorithmic approaches that were developed to cover these three topics. In addition to the presentation of the underlying geometric concepts, the validation results (computer-based verification and partially experimental validation) of the implemented algorithm are presented and discussed.

\subsection{GENERATION OF CURVED SLICES TO FILL A GIVEN VOLUME}

The need for curved slices arises from the geometry of the workpieces requiring repair. The planning very likely starts from a given cavity or volume with non-flat floor, from which the layers of new material will "grow." Since variating the layer thickness is an extremely difficult task, a uniform layer thickness is acceptable.

In Figure 125a, the CAD model of the geometry is presented with the steel blue color. The thick orange curve (1) on the top of the part represents the actual surface, while the dashed curves (2) and (3) represent the consequent curved layers. Since additive processes aim to apply multiple layers to actual surfaces to reach the target CAD model surface, the curved layers must not propagate outside the predefined boundaries, as shown in Figure 125b. The layers are generated with offset operations that ensure the generation of equidistant surfaces. Offset layers are not the same as the part surface. For example, radii change, edges and surfaces might disappear. The goal is to fill the entire repair volume. Then, the layers are trimmed to exclude surface patches outside of the given volumes. This technology tends to deposit an excess of material, since, in the most generic sense, you start with a part geometry with a curved surface and want to end up with a new part geometry with a new curved surface that is not $X$ times an offset. Therefore, further machining is often necessary. 
Figure 125: Generation of curved slices for repair

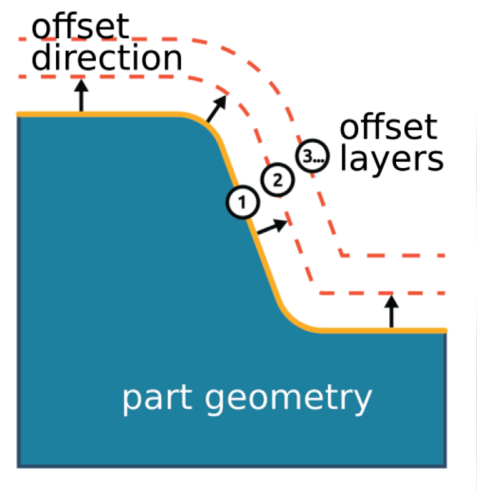

a) Offsetting from the base material

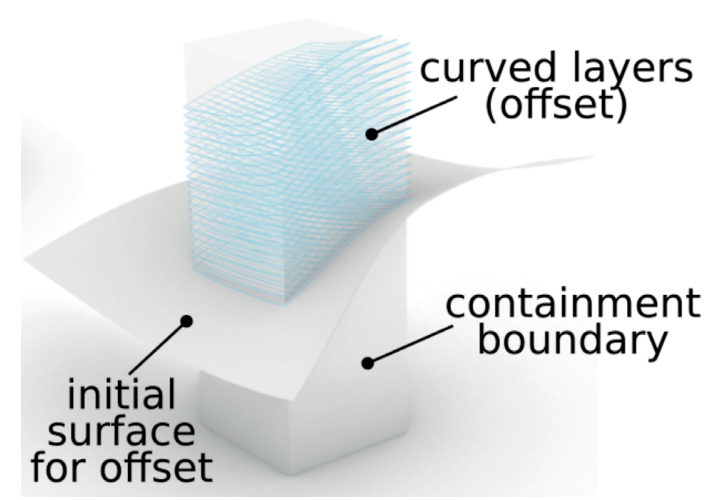

b) Trimming offset surfaces to the boundary

The data representation of the start surface mostly depends on the source of the CAD data. In the case of building from scratch, it is likely to be created in CAD modeling software. For repair applications, the source is a tessellated surface in stereolithography (STL) file format. The accuracy of a CAD model's geometry is superior to the accuracy of a measured model. CAD models mostly consist of multiple NURBS surfaces with curve tangency (C1-continuous), while the measurement results of 3D scans are triangle meshes representing $\mathrm{CO}$-continuous geometries, which means that the triangles are typically connected to each other, but they do not have curve tangency.

The mathematical definition of NURBS is much more complex than a triangle mesh, where three corner points define a triangle and the sum of all the triangles define the triangle mesh. The offsetting and trimming of NURBS surfaces and triangulated surfaces are implemented differently because of the varying nature of the input data.

\subsubsection{Offsetting and trimming of NURBS}

Unfortunately, it is not simple to generate an explicit offset of a given CAD geometry consisting of multiple NURBS surfaces . also investigates the complexity of this problem. Typically, a CAD geometry consists of multiple NURBS surfaces that are, in most cases, trimmed. The mathematical offset of a trimmed NURBS surface requires that an approximated trimmed NURBS at a given offset distance be identified. With all NURBS surfaces offset, the intersection of the offset surfaces needs to be calculated and, in some cases, fillets need to be added. Applying a triangle mesh offset algorithm will thus be more effective in terms of the algorithmic implementation effort.

Explicit offsetting of a single untrimmed NURBS surface is achievable. NURBS surfaces are mathematically polynomial and, analytically, they can be extended to represent the explicit offset surface. However, most NURBS surfaces coming from CAD software are trimmed sur- 
faces that typically do not touch each other to form a closed solid at the trim boundary next to neighboring surfaces. Instead, tiny gaps of overlapping regions are quite common due to the operating methods of CAD software and CAD engines.

NURBS surfaces have an advantage in terms of accuracy. In a CAD model, the NURBS representation of geometry can exactly match the intended CAD design, since the NURBS mathematics is based on polynomial definitions and continuity is mathematically given for polynemas. In contrast, a triangle mesh definition is always an approximation of a curved geometry, where the number of triangles increases as the need for accuracy grows. The use of NURBS directly in CAM applications is required when very high accuracy is important. On the other hand, as explained above, the cost of developing NURBS mathematics-based toolpaths is quite high due to the complexity of the topic. As such, triangle mesh-based toolpath calculation has become the de-facto standard in commercially available CAM applications for mold and die machining or production part machining.

\subsubsection{Offsetting of meshes}

It is worth mentioning that the offsetting of meshes can also be applied beyond repair applications. Sometimes, it is more economically viable to fabricate some features of the workpiece with, for instance, laser cladding, or to build using dissimilar or graded multicomponent materials. Figure 126a shows an example of the green base geometry of an actual part shape and gray target build-up geometry described as a triangle mesh. In order to build up the gray feature using multiple layers parallel to the green layer, a toolpath calculation algorithm needs to offset the green surface by a specific amount that is equal to the layer thickness and trims the result with the gray mesh. Figure $126 \mathrm{~b}$ presents this approach. The yellow containment curve in Figure 126b is the intersection curve of the offset with the gray target build-up geometry.

Figure 126: Generation of layers from offset surfaces

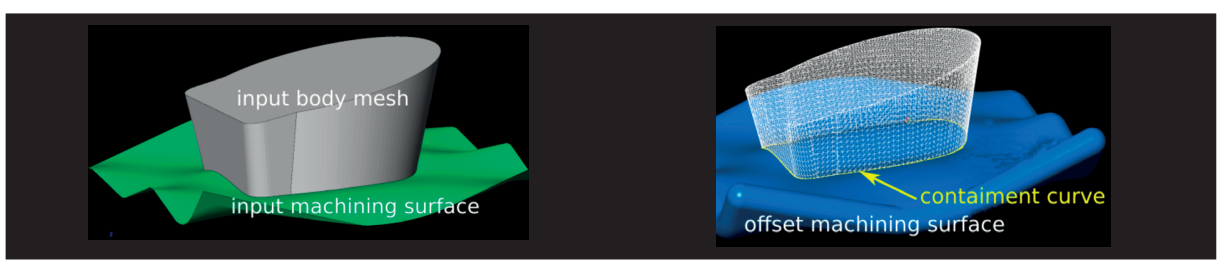

a) Build-up of arbitrary geometry shapes

b) Offset surface intersection with build-up geometry

Furthermore, the CAD/CAM operator needs to provide a drive curve to impose a toolpath pattern within the containment curve. The drive curve is used as an input for toolpath generation, whilst the containment defines a domain where the generated toolpath will exist. Figure 127 shows an example of a circular drive curve that generates a concentric toolpath, which is then trimmed outside of the containment boundary. Without a drive curve, the offset surface and 
containment curve are not enough to determine what the toolpath pattern should look like. A pocket toolpath, like deposition (as used in milling applications), within the containment curve will cause the welding head to move in a manner that is not very smooth, especially compared to a specially designed drive curve, where the welding toolpath is forced to always move parallel to the drive curve, creating a smooth motion and not running around the corners. Therefore, the AM toolpath calculation for each layer requires an understanding of the geometrical shape at each layer, including the surface definition, containment curve and drive curve. As explained above, all three of these geometric elements are specifically offset from the original geometry. The geodesic curve propagation algorithm will be described later in this chapter. The explicitly calculated triangle mesh must be, per definition, a 3D offset geometry of the original CAD geometry.

Figure 127: Relationship between the drive curve, containment and generated toolpath

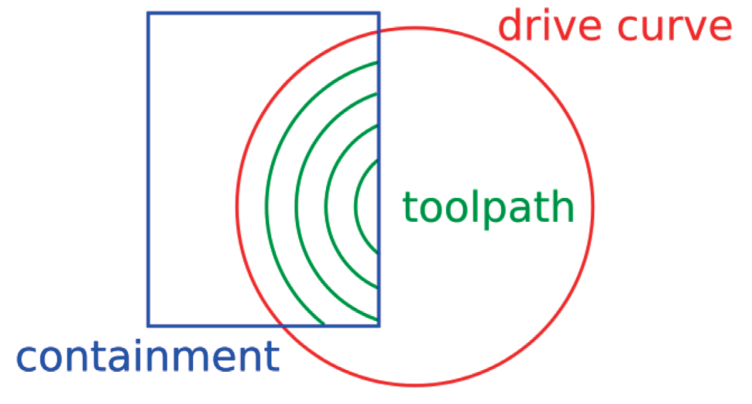

However, the offsetting of triangulated meshes is not an easy task. The easiest approach is to offset each triangle facet individually, then find the global envelope of all the offset triangles. The complexity of the envelope is considerably higher than the complexity of the mesh itself. As an illustrative example, let's consider computing the offset of a single triangle. The offset of a single triangle is a surface that is equidistant from it, meaning that the offset envelope is composed of several surfaces : two planar features parallel to the triangle, three cylindrical features defining equidistant loci from the three edges, and three spherical features defining equidistant loci from three vertices, as shown in Figure 128a-Figure 128c. Although planar, cylindric and spherical features are rather simple, the resulting surface of the offset, which is shown in Figure 128d, is substantially complicated and requires the calculation of borders between the primitives. Moreover, the offset surface should be tessellated in order to be represented as a mesh. Depending on the accuracy of tessellation, an offset mesh of a single triangle may consist of hundreds of triangles. 
- Figure 128: Generation of a triangle offset

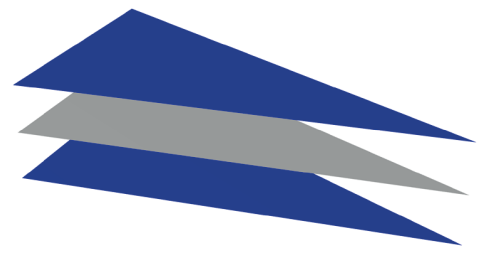

a) Triangle (grey) and two parallel triangles (blue)

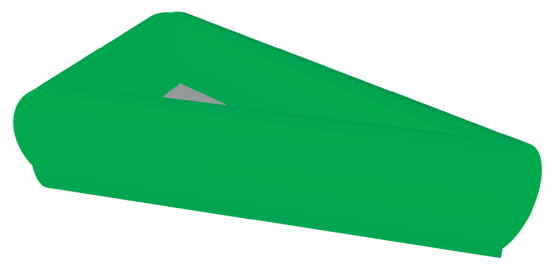

c) Triangle (grey) and three cylinders at the edges (green)

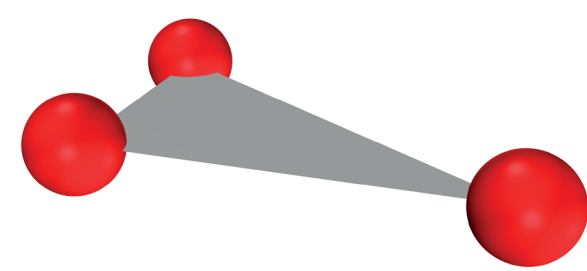

b) Triangle (grey) and three spheres at the vertices (red)

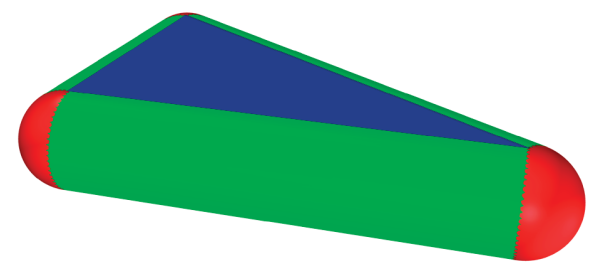

b) Offset primitives combined

Offsetting a mesh consisting of multiple triangles is more complex than offsetting each individual mesh triangle. In a typical case, the mesh triangles are close enough to each other that offsets of the triangles intersect with each other. Therefore, to find the outer hull of all of the offset meshes, it is also essential to exclude self-intersecting volumes, i.e., surfaces that are inside of the offset meshes. Additionally, there is still the challenge of numerical stability. Since computers use limited precision ( 7 or 15 decimal digits) for mathematical operations-such as addition, multiplication and others-it is inevitable that the result will have certain numerical errors. For example, finding the intersection point of two almost parallel lines is very numerically complex. Since most of the offset triangles are almost parallel to each other, this leads to numerical issues, even though the analytical definition of the problem is very sound and deterministic. Furthermore, there is no guarantee that offsetting two neighboring triangles and resolving the overlap or gap between them will generate the desired offset triangle mesh, since other triangles not directly neighboring two processed triangles can also generate intersections with them. This leads, at least theoretically, to the need for all offset triangles to be checked against all other offset triangles, which requires a significant computation effort that grows quadratically with the number of triangles.

This problem has been tackled differently by different researchers. suggested a smarter procedure that takes multiple normal vectors at vertices into account to generate spherical and cylindrical surfaces. However, more advanced volumetric methods are based on distance volumes and the fast-marching method and. Voxel-based approaches could potentially tackle the 3D offset problem with a different algorithm that first generates an implicit space defi- 
nition where $d(x, y, z)$ must be the offset distance of a given input mesh to any given point in space. Then, a triangle offset mesh based on the implicit offset definition is generated. Figure 129 illustrates how these methods work on regular voxel grids. Instead of computing offset surfaces, each voxel attributes a certain value computed as the shortest distance to the given mesh, which is discretized with voxels.

Figure 129: Signed distance field to voxels

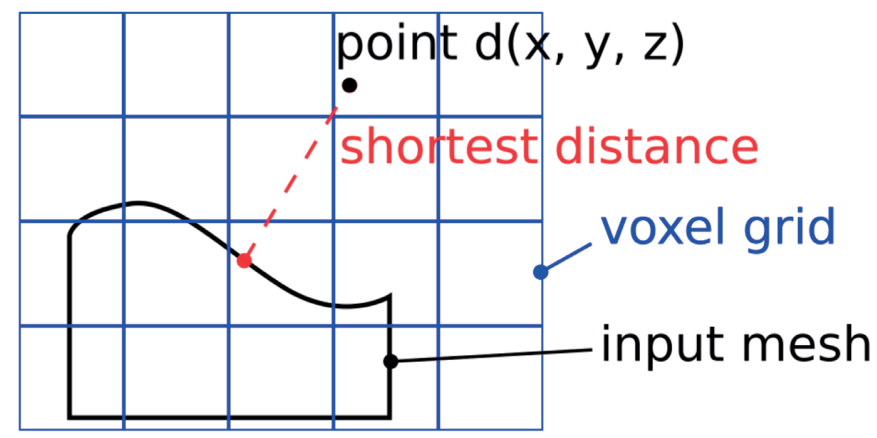

By definition, $d(x, y, z)$ is a continuous function in the three dimensions, since every finite change in $x, y$ or $z$ will change the distance of the point $\boldsymbol{P}$ at the coordinate $x, y$ and $z$, with a finite increase or decrease in relation to the $x, y$ and $z$ change. But the voxel grid is a discrete manifold. Therefore, the shortest distances assigned to them cannot create a continuous field that can be converted into isolines, i.e., offset surfaces. Thus, an approximation with marching cubes is used to restore iso-surfaces within voxels.

In their previous work extended linked voxel structures to point-to-mesh distance computation, which were developed to derive equidistant mesh surfaces. Equal size voxels, shown in Figure 130, were used for a spatial partitioning on volume rasterization for collision detection in the context of multi-axis machining. Regardless of the application, the math behind the computation is designed to compute the distance between a mesh and spatial points. The voxel data structure was enhanced by establishing links to empty cells that support efficient point-to-mesh distance queries. The advantage of organizing the objects in cells of equal size instead of cells with adaptive size (such as in k-D trees, octrees, R trees, or BSP trees) is the fact that all cells have the same shape and scale. This assumption allows specific criteria that apply equally to all cells to be formulated. Treating all cells equally allows for pre-computing, which in turn makes the process more efficient. 
- Figure 130: Example of a discrete voxel sphere

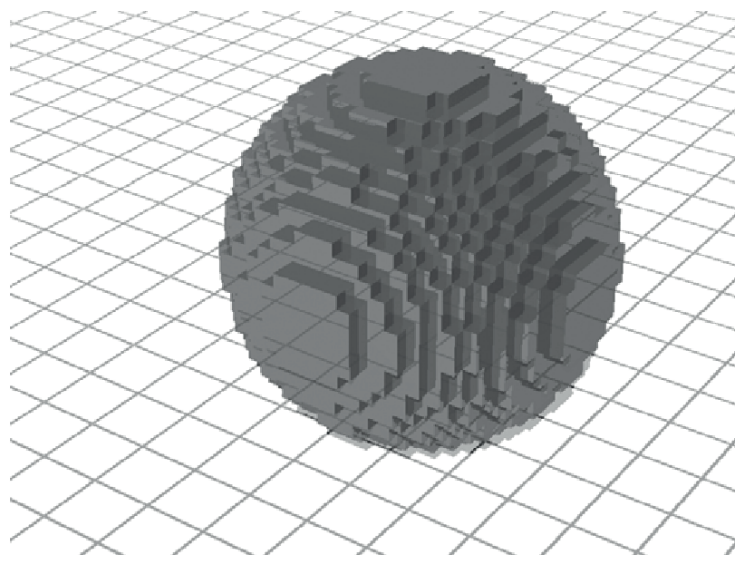

\subsubsection{Linked voxel structure}

Given a rasterization step size of $s$, the voxels represent non-overlapping cubes with a side length of $s$. For each voxel, a set of local triangles is stored in a local BSP tree (binary space partitioning the trees introduced in ). BSP trees contain triangles in a data structure that recursively subdivides the space into an almost equal number of triangles in each subdivision. For "empty" voxels, a list of links is stored to select nearby non-empty voxels. Each voxel supports an efficient query of the distance to a local set of triangles. The structure and attributes of a voxel in linked voxel structures are outlined in Figure 131. If the voxel contains triangles, the triangles are organized in a BSP tree (triangleArray). Otherwise, the surrounding non-empty voxels are stored in a list (voxelArray). In the following section, the attributes and properties of a linked voxel structure are discussed.

Figure 131: Class diagram of a basic voxel object

\begin{tabular}{|l|}
\hline \multicolumn{1}{|c|}{ Voxel } \\
\hline -triangleArray: bsp-tree \\
-voxelArray: list \\
\hline +getDistance(pt:3dPoint): distance \\
\hline
\end{tabular}

A voxel is deemed "empty," if the circumscribing sphere touching all eight corners of the voxel contains no triangles. Otherwise, the voxel is called "non-empty." The radius of this sphere is half the length of the voxel's diagonal. If $\delta$ is the voxel width, then the radius of the sphere is $\delta \sqrt{3} / 2$. Figure 132 depicts the $2 \mathrm{D}$ analogon, while the small black circle illustrates the radius. 
A voxel is deemed non-empty if the surrounding sphere (small, dark circle) contains triangles. All triangles contained in the large, light circle are assigned to the light gray, non-empty voxel.

Figure 132: Example of empty (black) and non-empty voxels (light gray)

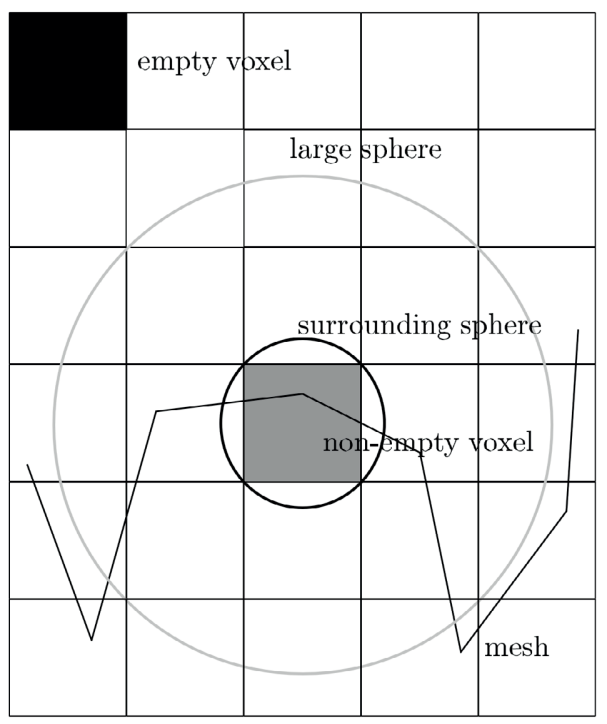

The basic idea of obtaining efficiency in the point-to-mesh distance computation for a point $\boldsymbol{P}$ that lies in a voxel $V$ is to have all required information for the distance computation associated with that single voxel $\mathrm{V}$. This allows for local processing of the query.

Consequently, non-empty voxels contain all the triangles that (fully or partially) lie within a sphere with a radius of $3 \delta \sqrt{3} / 2$ whose center is the barycenter of the voxel. For any point $\boldsymbol{P}$ lying in a non-empty voxel $\mathrm{V}$, we know that the distance to the mesh is smaller or equal to the length $\delta \sqrt{3} / 2$ of the diagonal of the voxel. Considering all triangles within a sphere with a radius of $3 \delta \sqrt{3} / 2$ ensures that each voxel simultaneously contains all the information necessary for the point-to-mesh distance computation for point $\boldsymbol{P}$ and as little information as possible.

If point $\boldsymbol{P}$ lies in an empty voxel, one needs to consider all triangles of the closest non-empty voxels for the point-to-mesh distance computation. Hence, each empty voxel gets assigned a list of links to the closest non-empty voxels. 
Setting up a linked voxel structure involves three steps:

1. Determining non-empty voxels (surrounding sphere criterion).

2. Filling non-empty voxels (large sphere criterion).

3. Assign to each empty voxel the closest non-empty voxels.

For filling the non-empty voxels, the algorithm iterates over all triangles and computes the voxels the triangle belongs to, whether fully or partially, for each triangle. Hence, whether one of the triangle's vertices lies within the sphere circumscribing the voxel (surrounding sphere in Figure 132) is checked. In a second iteration, the triangles lying within the associated extended sphere (large sphere in Figure 132) are assigned to each non-empty voxel. All triangles assigned to a non-empty voxel during the two iterations are stored in a local BSP tree.

When assigning non-empty voxels to empty voxels, the closest and most appropriate sets of non-empty voxels must be determined. Since the distance between an arbitrary point, a voxel $\boldsymbol{P}$, and the mesh is to be computed, the linked voxels must contain all the information required for the distance computation. This means that the closest triangle to that arbitrary point must lie in one of the linked non-empty voxels. To estimate an appropriate set of linked non-empty voxels, discrete spheres with radii $\delta r$ with $r \in \mathbb{N}$ around the empty voxel are considered. The computation of the discrete spheres is presented in Figure 133. If $\delta r$ is the smallest radius, links to all non-empty voxels contained in the discrete spheres of radius $\delta r, \delta(r+1), \delta(r+2)$ and $\delta(r+3)$ are added to the list for the current empty voxel.

The discretization error makes it necessary to look into these three subsequent spheres. Figure 134 illustrates this observation for the 2D analogon: Consider a sphere (black arc) with a certain radius $\delta r$ around a cell (gray with black border cell). Its discretization includes all the dark gray and the striped light and dark gray cells. The striped cells indicate that successive discrete spheres may overlap. When looking at the cell with the white frame, it becomes obvious that a triangle located at the far side of that cell (indicated by the gray line) is farther from the gray voxel than certain parts of some light gray cells. The distance from this triangle (gray line) to the sphere (black arc) is bounded by the length $\delta \sqrt{3}$ of the diagonal of a cell. Hence, triangles within a distance of up to $\delta(r+\sqrt{3})$ must be checked. Moreover, the sphere is centered at the gray cell's barycenter (black dot), whereas the actual point of interest may lie at the corner of the cell (white dot). The black and the white dot in the empty voxel has a maximum distance of $\delta \sqrt{3} / 2$. Taken together, triangles up to a distance of $\delta(r+3 \sqrt{3} / 2) \approx \delta(r+2.6)$ must be considered. 
Figure 133: Algorithm calculating a discrete sphere

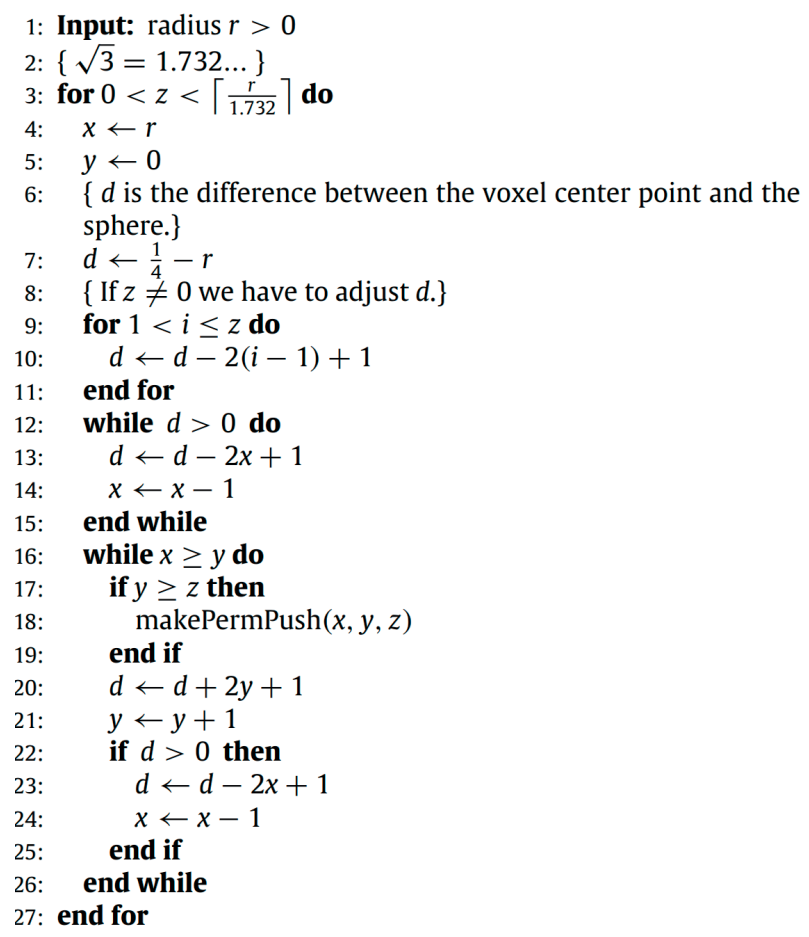

Figure 134: Considering the enabling of an empty cell (gray cell with black border)

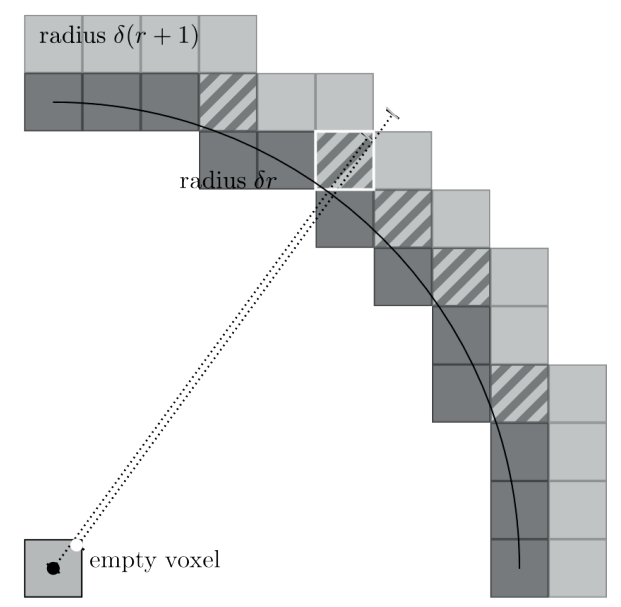


The expected time complexity of the initialization step that sets up the linked voxel structure is linear in the number of triangles. When choosing the voxel size so that the number of voxels depends on the number of triangles, e.g., proportional to the cube root of the number of triangles, the number of triangles per voxel is expected to be constant. Each non-empty voxel stores the triangles in a BSP tree. Since the expected number of triangles per voxel is constant, the construction of the BSP tree is constant in time. In the last step, each empty voxel is assigned to the closest non-empty voxel. For determining the closest non-empty voxel, it is expected that only a constant number of surrounding voxels need to be considered. Since the number of voxels is chosen in order to be linear in the number of triangles, the time complexity of the last step is also bounded linearly by the number of triangles. Hence, the overall expected time complexity is expected to be linear in the number of triangles. This linear time complexity makes it feasible to apply our approach to surfaces represented by large triangular meshes.

\subsubsection{Point-to-mesh distance computation}

The distance from any point in the 3D space to the mesh can be efficiently calculated with the linked voxel structure. The resulting code for the getDistance function is presented in Figure 135.

- Figure 135: Pseudo-code of the GetDistance function

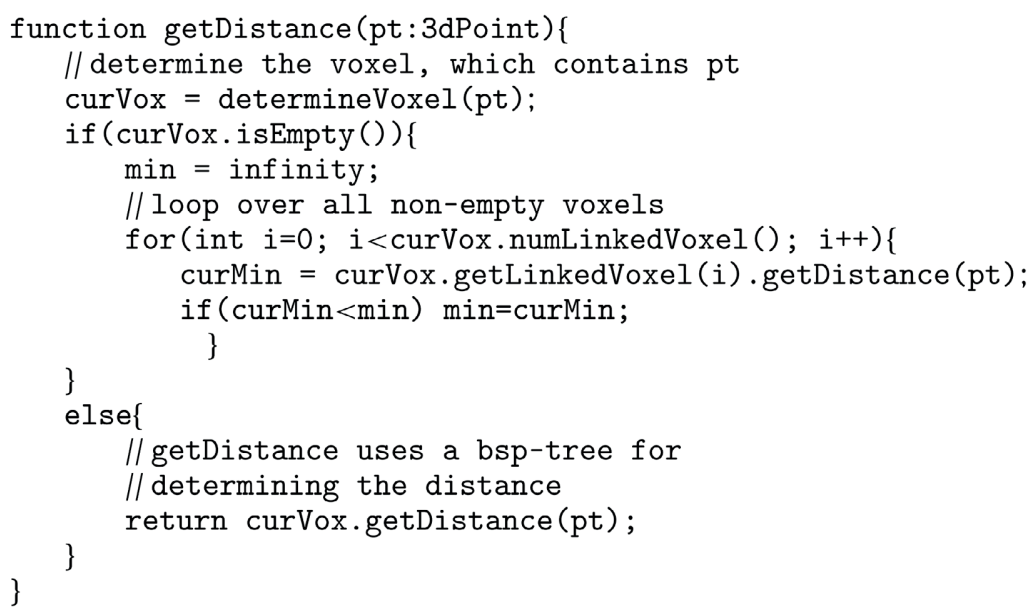

The first step in the point-to-mesh distance computation algorithm is to determine for the given point $\boldsymbol{P}$, the voxel $\mathbf{V}$ to which $\boldsymbol{P}$ belongs:

1. If $\mathrm{V}$ is non-empty, the minimum distance of $\boldsymbol{P}$ to any of the triangles stored in the voxel $\mathrm{V}$ is calculated by calling its associated getDistance function. As the set of locally stored triangles is expected to be small, this computation can be completed quickly. It is made even faster due to the use of a local BSP tree. 
2. If $\mathrm{V}$ is empty, a loop over all the linked voxels from the current voxel and a call of their associated getDistance function provides a list of distance values between $\boldsymbol{P}$ and the triangles stored in the corresponding linked voxel. The smallest value indicates the minimum distance between $\boldsymbol{P}$ and the mesh.

\subsubsection{Triangulation with adaptive extended marching cubes}

The task of calculating an offset triangle mesh at a distance $d(x, y, z)=$ const is similar to a computer tomography (CT) application, where the sensor delivers a three-dimensional data set of grayscale values, and the so-called "iso-surface extraction" is the process of explicitly using a triangle mesh calculation at a given grayscale threshold value. The requirement here is very similar to this application. The difference, however, is that the sensor accuracy is limited to a number of cells where the grayscale value is calculated, whereas the distance field $d(x, y, z)$ can calculate the exact distance value for any point in space.

For CT applications, an algorithm called "the marching cube" has been developed to generate a triangle mesh based on a given grayscale threshold value. Since the application requirements are similar, the marching cube algorithm can be considered an appropriate and robust method to extract an explicit triangle mesh when an implicit space definition like $d(x, y, z)=d_{\text {offset }}$ is present.

The main idea of the marching cube algorithm is the approximation of a boundary (contour or surface, depending on the dimensionality) based on a grid, in which the grid nodes can have only two statuses (inside or outside). Figure 136 shows a 2D case of marching cubes. Depending on the constellation of inside and outside nodes, different approximations are possible, as shown in Figure 136a. Therefore, the circle in Figure 136b is approximated with the octagon in Figure 136c and consists of piecewise approximations provided by each cell.

Figure 136: Marching cubes in 2D (Boris, 2018). Inside nodes (black), outside nodes (white).

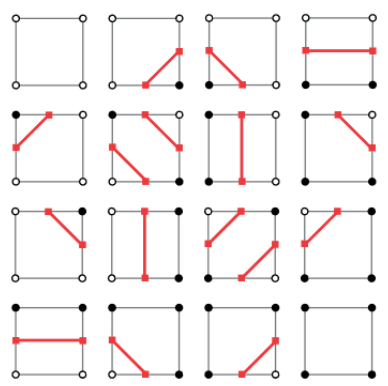

a) 16 possible 2D approximations b) Circle and the grid (red)
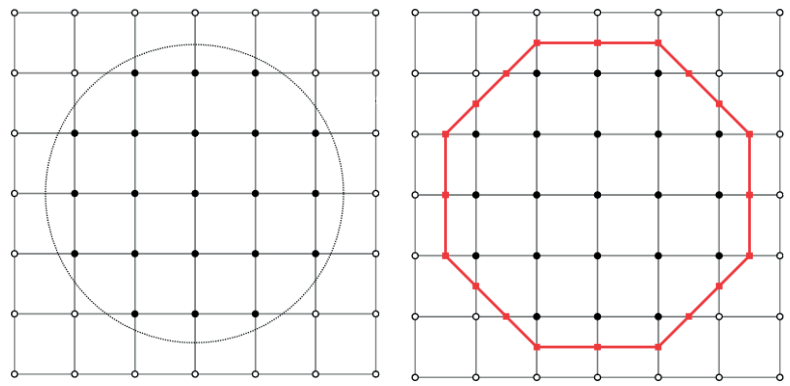

c) Circle approximation (red) 
To support mesh offsetting, the linked voxel structure also contains the distance values from the mesh to the eight corners of the cell. This computation can be done efficiently using the point-to-mesh distance computation described in the previous section (4.1.2.2).

Knowing the distance values at the voxel vertices, it is possible to identify whether or not the offset surface exists in a voxel. The rule is simple: some distances at vertices must be less than the offset value, and other distances must be larger than the offset value. Depending on the distance, the voxel vertices are marked as inside/outside nodes. Then, the offset surface can be restored with the 3D marching cubes algorithm, which is the 3D version of the approach depicted in Figure 136. Considering only one voxel on its own, the mesh offset algorithm creates surface patches within each voxel. The collection of surface patches from each voxel eventually yields a triangle mesh approximation of the complete surface.

The positions of the endpoints of the approximated lines are fixed at the middle points of the edges. However, this approximation can be improved because the voxel nodes have distance values, which allows the best position for the approximated points of the edge-offset intersection to be calculated, as shown in Figure 137a. In cases where accuracy requirements (for instance, a threshold for the difference between distances at neighboring nodes) are not met and there is a need for recalculation, a finer triangulation within the cube, as shown in Figure 137b, can be achieved, as in .

An interesting benefit of the described approach, in contrast with a triangle-based offset, is that this method does not require additional effort in terms of fixing problems with the initial mesh (gaps, overlapping triangles, etc.). Healing an input mesh comes at no extra cost. The function $d(x, y, z)$ deliberately ignores any irregularities within the input mesh because, mathematically, distance calculation in space is well behaved, continuous and without singularities.

Figure 137: Marching cube approximations for an offset distance=1. The values at the nodes are the distance field values.
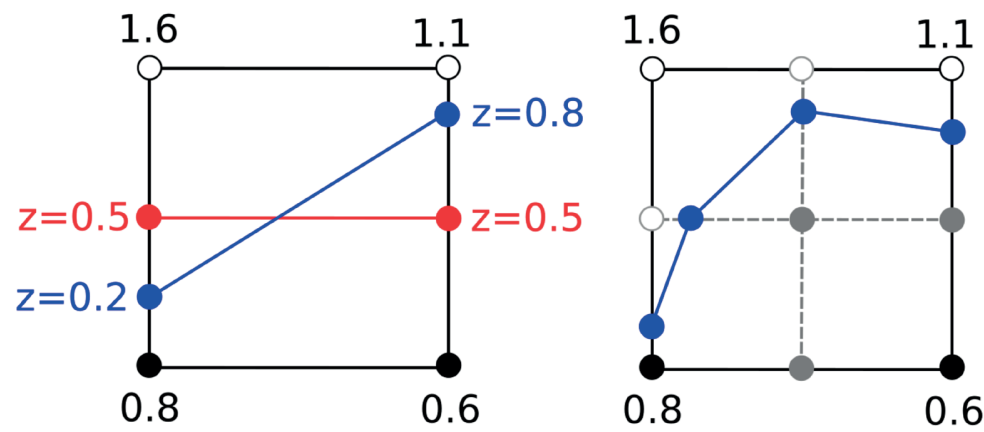

Orefined onodes

a) Classic (red) and extended (blue) marching cube b) Refined grid and refined approximation (blue) approximations 
One known drawback of marching cubes techniques in general is that they do not take the local surface curvature into account, i.e., the connection surfaces within voxels are flat. In this implementation, applying sophisticated refinement criteria guarantees the required mesh accuracy will be achieved. Refinement leads to more computations, but, since each voxel is processed independently from the others, this extended marching cubes code was parallelized for computation speed and to overcome the refinement overhead.

\subsubsection{Examples of the mesh offsetting algorithm}

The algorithm developed has been implemented in C++ environment. The resulted offset meshes can be exported in STL format or visualized using OpenGL rendering. Figure 138 shows an example of a mesh offset generated by the developed method.

Figure 138: Example of the implemented mesh offsetting

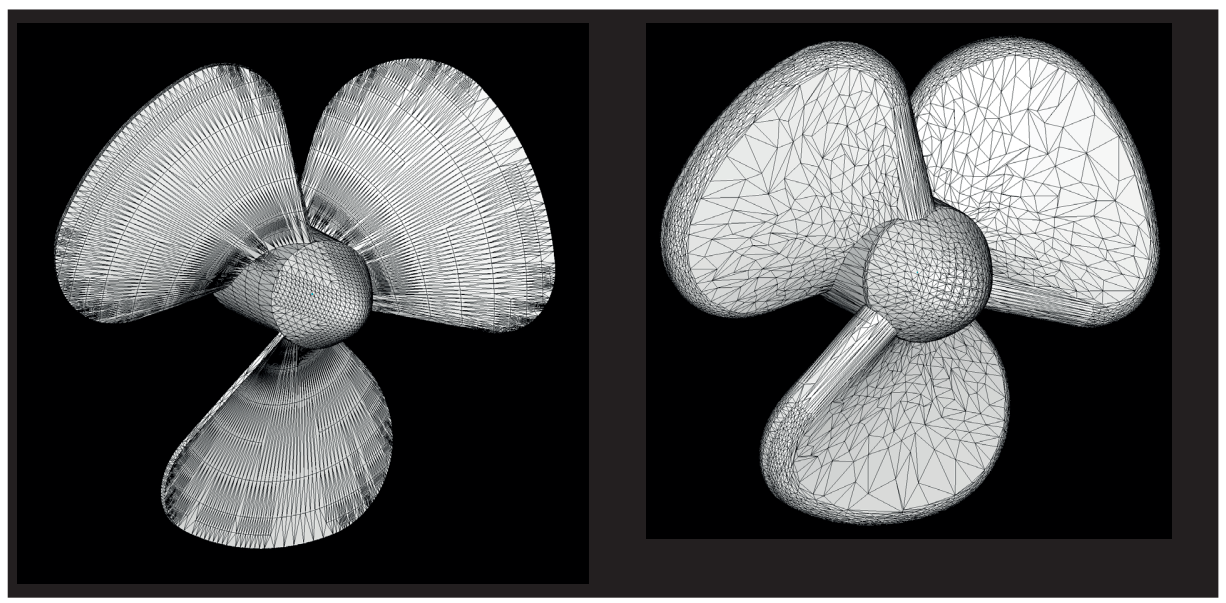

a) Mesh of a propeller

b) Offset mesh (decimated to reduce the number of triangles)

Sometimes it makes sense to use different offset values for some of the surfaces of a mesh. Therefore, the developed marching cubes approach has been extended to support multiple distance functions to nodes, alternating between them to choose the ones that affect the offset calculation. Figure 139 depicts an offset with different offset distances assigned to different surface patches. 
- Figure 139: Example of offsetting with a variable offset distance

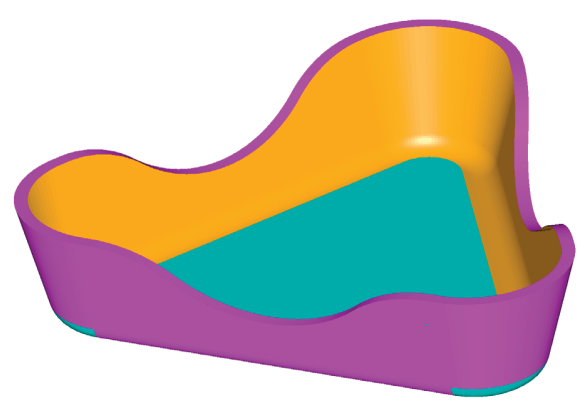

a) Initial mesh with different offset distances assigned to surfaces with different colors

\subsection{Generation of toolpaths on curved slices}

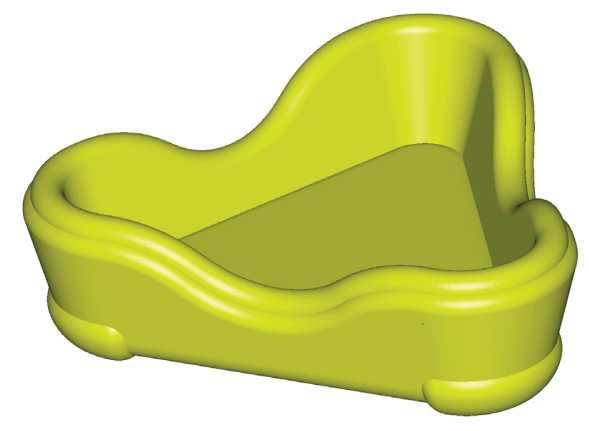

b) Offset mesh

While depositing a curved layer, it is important to ensure that the material is deposited with uniform thickness. Therefore, cladding toolpaths must guarantee a constant distance between adjacent toolpaths to prevent gaps and variable overlaps of toolpath tracks. Thus, all toolpaths should be iso-distant-also known as geodesic-i.e., they must have constant a stepover (distance) between the tracks of the toolpath. Generation of a geodesic toolpath requires a starting (also called a guide, base or drive) curve that is used as a reference to generate the subsequent curves, as shown in Figure 140. The starting curve must either be provided by the user or be a feature of the layer, such as the mesh center or its boundary.

Figure 140: Iso-distant (geodesic) curves

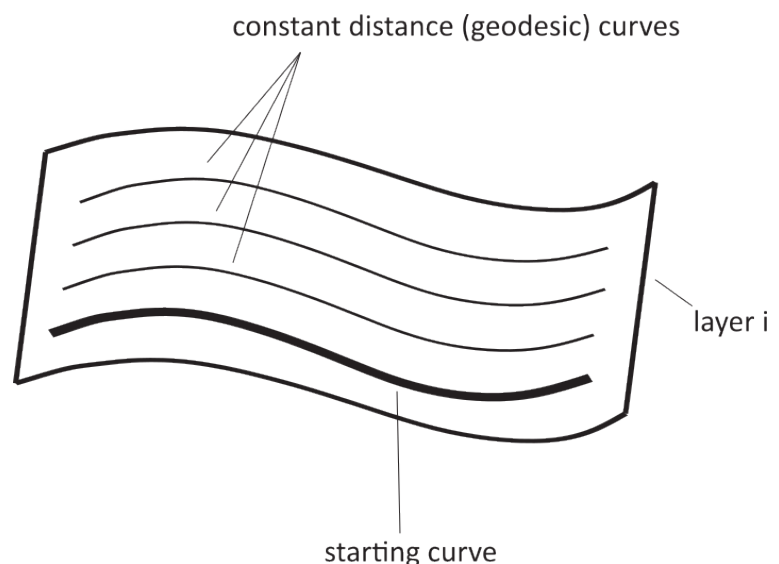


Since additive toolpaths were and still are based on the advances in subtractive toolpath calculation, the generation of additive toolpaths is inevitably connected to milling strategies. Geodesic methods for milling were originally developed more than a decade ago (, ). These technologies were applied to mesh geometry, especially in the die and mold industry.

The most advanced approaches are based on global distance fields. A global distance field without a fixed direction enables full flexibility in terms of calculating various pattern types while maintaining consistent distances between cuts for single OR multiple guide curves.

The exact calculation of geodesic curves on triangle meshes was introduced in , and further elaborated in . To easily understand geodesic curves, here a short abstraction. Imagine a surface made out of water. You throw guide curves into the water, which generates ripples on the water. Now, the top of each wave has a distance to the previous wave defined by the maximum stepover. Those geodesic distances are used to create patterns that consider the distances of the surface topology, as shown in Figure 141.

Figure 141: Pattern propagation on surfaces (from left to right)

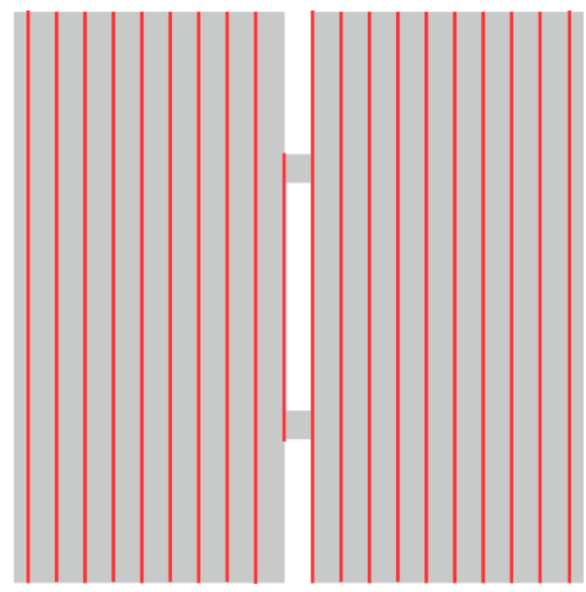

a) No consideration of topology or gaps

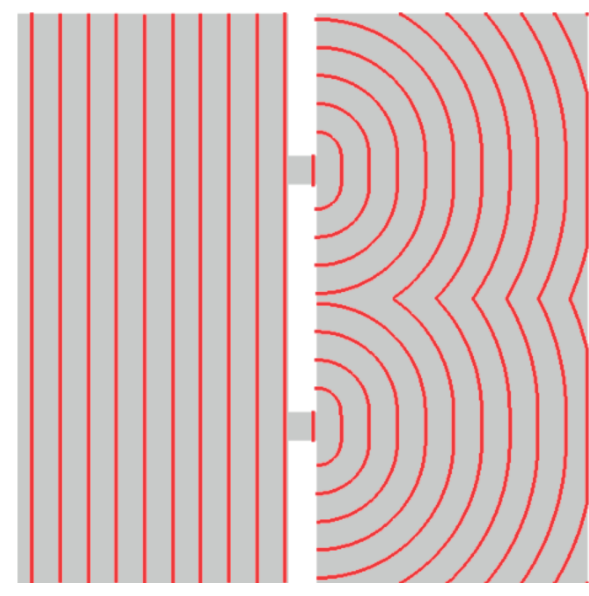

b) Considering the actual mesh topology

In order to maintain computational efficiency, the mesh is enriched with an additional graphlike data structure to store the distances from the base curve of the selected points of the triangle edges. The geodesic distance field is computed as follows. For each baseline of the guide curve, as depicted in Figure 142, the edges of the closest triangle are split into segments, which are called "windows". 
Figure 142: Principles of geodesic computation

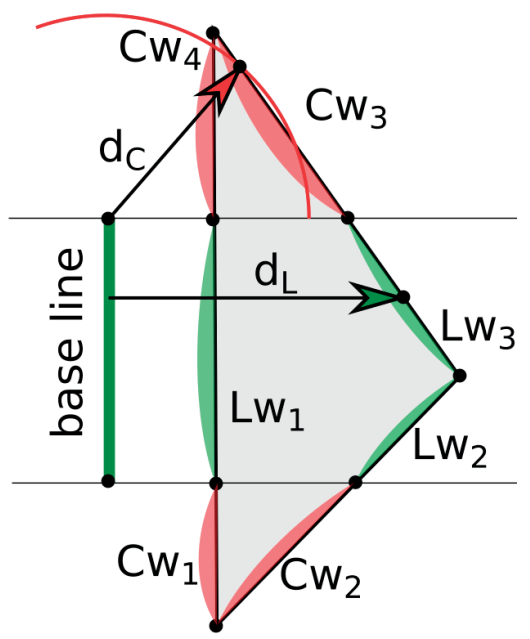

a) First triangle

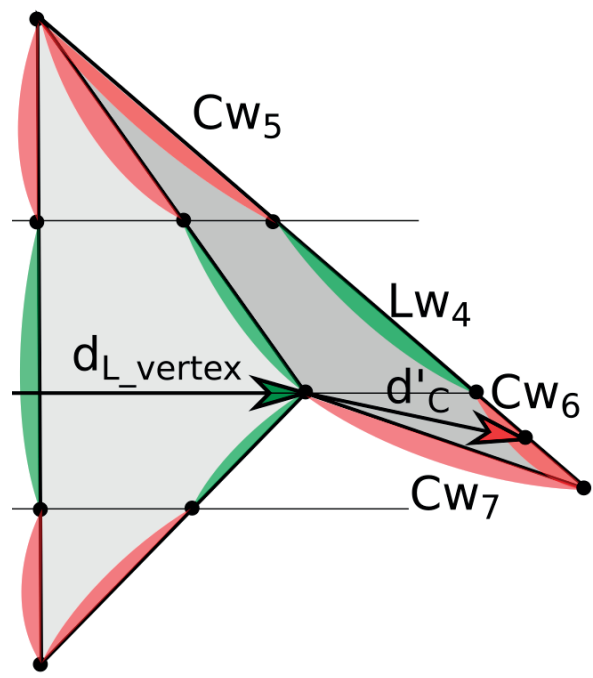

b) First and second triangles

The key characteristic of each window is how the shortest distance from the baseline to points on the windows is searched. The linear windows (green) $\left(L_{W 1} \ldots L_{W 4}\right)$ are the intervals, so the distance $d_{L}$ from the baseline to a point on the linear window is the projection of the point on the baseline in the $2 \mathrm{D}$ space of the unfolded mesh. Within a linear window, $d_{L}$ is a linear function of a point on the linear window. In contrast, the circular windows (red) $\left(C_{W 1} \ldots C_{w 7}\right)$ define a distance function $d_{C}$ from some critical points as a radius vector. Figure 142 a shows $d_{C}$ positioned at an endpoint of the baseline, while Figure $142 \mathrm{~b}$ shows $d_{C}{ }_{C}$ positioned at a triangle vertex. The distance field can be visualized by coding distances with different colors at points on the surface, as shown in Figure 143. 
Figure 143: Visualization of the distance field

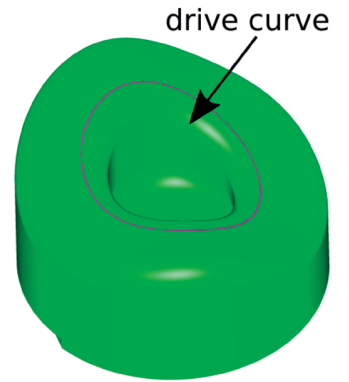

a) Input surface and the drive curve

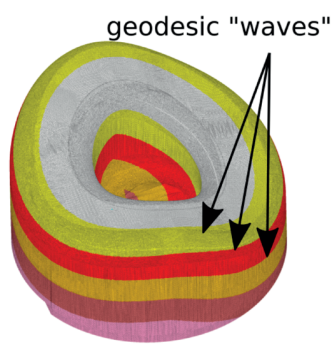

b) Colored distance map on the mesh

One of the biggest advantages is the flexibility of applying the technology to different kinds of geometry features in any combination, as shown in Figure 144. Single or multiple guide curves can be used as an input. Even in an undercut situation, the algorithm outputs the same consistency.

- Figure 144: Example of a geodesic toolpath. The outer contour is the drive curve. Geodesic offsetting is inward.

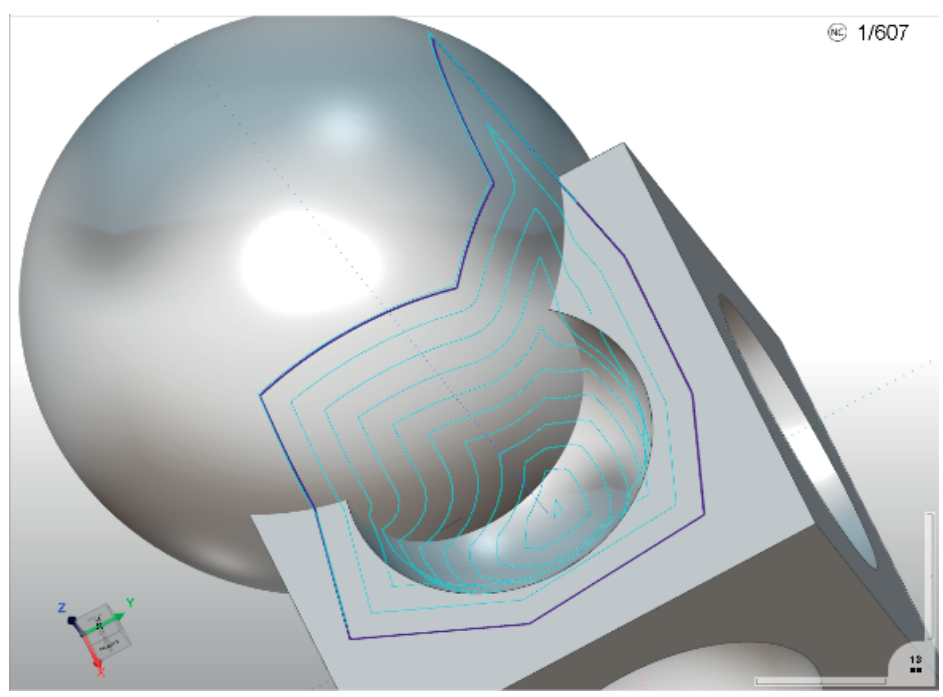




\subsection{PLANNING OF THE NOZZLE ORIENTATIONS FOR DED}

Defining the nozzle orientation is a critical issue in multi-axis AM. The rule of thumb is to keep the deposition normal to the curved layer, as shown in Figure 145. Sometimes, this is not feasible because of the presence of collision obstacles, especially in repair applications. Even if there is no collision potential, this approach is not always a good one because of the limitations of the process, for example physical limits of nozzle tilt angle. Other issues may also arise like geometry and local surface normal changing frequently along the path of the nozzle such the acceleration of the kinematics might not be able to handle such changes. These and other similar topics are discussed in the following sections.

Figure 145: Nozzle positions oriented normal to the surface

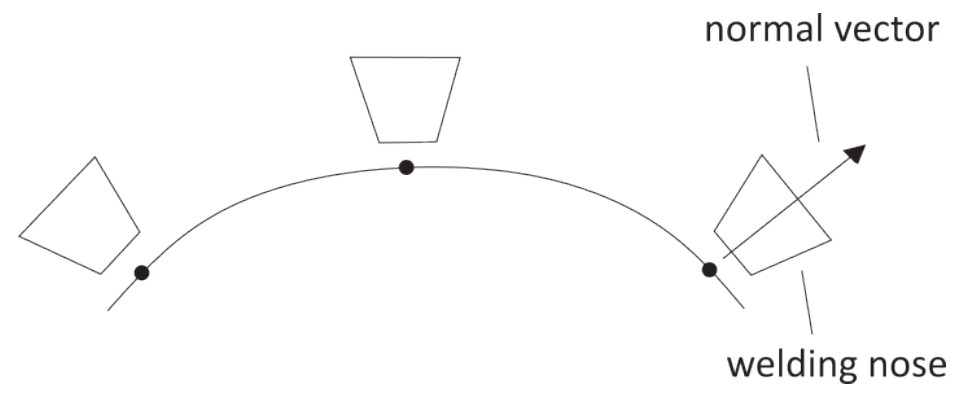

\subsubsection{Redistribution of toolpath points and initialization of a collision-free toolpath}

Toolpaths for AM applications may consist of millions of points. Finding smooth and collision-free tool orientations in a brute-force manner is almost guaranteed to take several hours or days. In order to avoid potential problems, namely collisions and abrupt orientation changes, while maintaining a normal-to-surface orientation as much as possible, a new algorithm called "auto tilting" has been developed. This algorithm finds collision-free orientations for each position while generating a smooth orientation change for subsequent positions.

The time required to find proper tool orientations can be shortened by precomputing the tool orientations at so-called "key points." These key points are the points at which the toolpath changes its direction or curvature significantly. If the distance between two neighboring key points exceeds a given threshold, additional key points will be inserted, as shown in Figure 146. 
Figure 146: Point types in DED toolpaths

- key points

- inserted key points

- sampled points

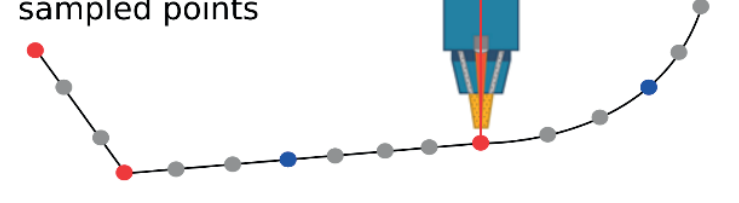

Collision-free tool orientations at key points are computed. The collision check is based on finding the Hausdorff (shortest) distances between the meshes. There are two levels of collision, shown in Figure 147, that can be checked:

1. Collision of the welding nose, which is represented by a simplified rotational geometry;

2. Collision of the complete kinematics of a CNC machine or robot arm.

It is obvious that the computational effort required for the first level of a collision check is considerably lower than for the second level. However, the limited first-level collision check requires that the calculated toolpath be verified in a further simulation step where the kinematics of the actual hardware (CNC or robot arm) is checked. This may require user-induced iterations in terms of changing the different parameters of the process planning, while a collision-free toolpath can be validated by a subsequent simulation.

Figure 147: Primitives used for different collision tests

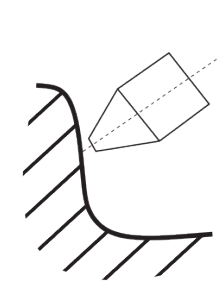

1) Collision test without kinematics

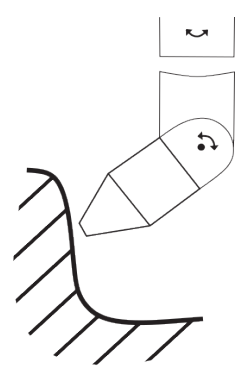

2) Collision test with kinematics 
Collision avoidance is done via variation of the nozzle orientation. If there is a collision of the tool when normal to the surface, the lead/lag angles are adjusted incrementally by one to three degrees until the collision is avoided. If a collision cannot be avoided, the search continues in the collision-free search domain (with a side tilt). This strategy is chosen based on the assumption that the laser spot can be either a circle or ellipse, as shown in Figure 148. In general, "stay normal to surface" should result in a perfectly circular spot. Any deviation from this will result in a deformed spot. If tool orientation changes are needed, tilting of the feed direction is preferred as this does not alter the width $d_{L F 1}$ in relation to the intended/original laser width $d_{L F O}$. Changes to the focus area might have secondary effects beyond just the change of the shape from circular to an ellipse. Such effects can be significant for the process and need to be further evaluated by future research.

Figure 148: Laser spot shape and dimensions depending on whether lead/lag and tilt are applied
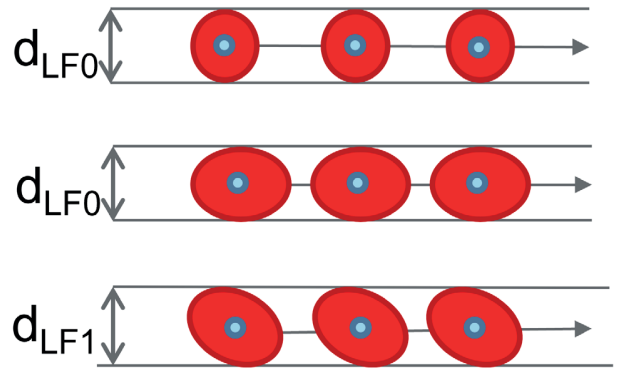

No tilting in feed direction

Lead/Lag in feed direction Lead/Lag and Tilting in feed direction

Depending on the sampling distance parameter, the number of sampling points (points between key points) on the toolpath changes. A large number of sampling points will impact the calculation speed significantly, but simultaneously, the difference in quality might not be critical. The orientations at sampling points are interpolated with SLERP interpolation, as demonstrated in Equation (22). SLERP interpolation is a spherical interpolation of the constant-speed motion of a vector along a great circle arc, given the ends and an interpolation parameter between 0 and 1 . The distance between two key points is parametrized to [0..1], and each sampling point has the coordinate $t$ within this interval.

$$
\operatorname{slerp}\left(v_{0} ; v_{1} ; t\right)=\frac{\sin [(1-t) \theta]}{\sin \theta} v_{0}+\frac{\sin [t \theta]}{\sin \theta} v_{1}
$$

, where $\boldsymbol{v}_{\mathbf{0}}$ and $\boldsymbol{v}_{\mathbf{1}}$ are the orientations at the first and second key points;

$\boldsymbol{t}$ is the interpolation parameter of a sampling point;

$\theta$ is the angle between $\boldsymbol{v}_{\mathbf{0}}$ and $\boldsymbol{v}_{\mathbf{1}}$. 
In most cases, all interpolated positions between two key points are also collision-free. However, there is the possibility that a collision may still happen. Therefore, the nozzle orientation at sampling points must be checked against collisions and, if necessary, a more feasible orientation must be identified. Every reorientation initiates a SLERP reinterpolation of the nozzle orientation at all sampling points-for example, if the reoriented sampling point acted as a key point. This procedure may run iteratively, adjusting the orientation at sampling points until all positions satisfy the collision conditions. Tool positions will be checked for collisions every three degrees (angle step of the tool axis). If there are areas along the contour where the tool is very close to the geometry, then a three-degree step might be too rough to find an optimal collision-free orientation and the step must be decreased.

\subsubsection{Toolpath smoothing}

In the case of significant changes to the tool orientation, as shown in Figure 149, the nozzle might slow down due to the jerk limits of the rotary axes. Hence, the surface might be overexposed to the deposition during the move. In order to prevent such cases and smooth the orientation changes, the orientation vectors are not allowed to have an angle change between sampling points that is greater than a specified value. For generic cases, the standard three degrees is usually suitable. These quick changes of orientation in short time intervals are not desirable since robotic or CNC kinematics may not be able to cope with such quick orientation changes, which will result in a slowing down of the welding nose movement. Since the deposition rate is typically kept constant throughout the process, this decrease in speed could result in more deposition of material than desired, as shown in Figure 150.

Figure 149: Significant orientation change during a move
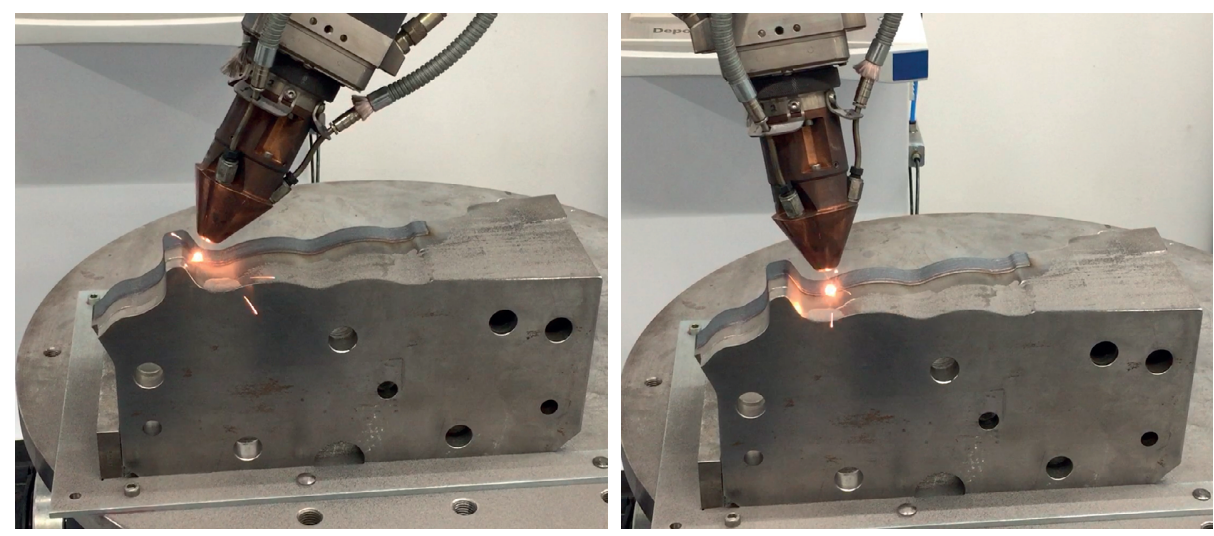
Gigure 150: Material over-deposition due to hardware kinematics

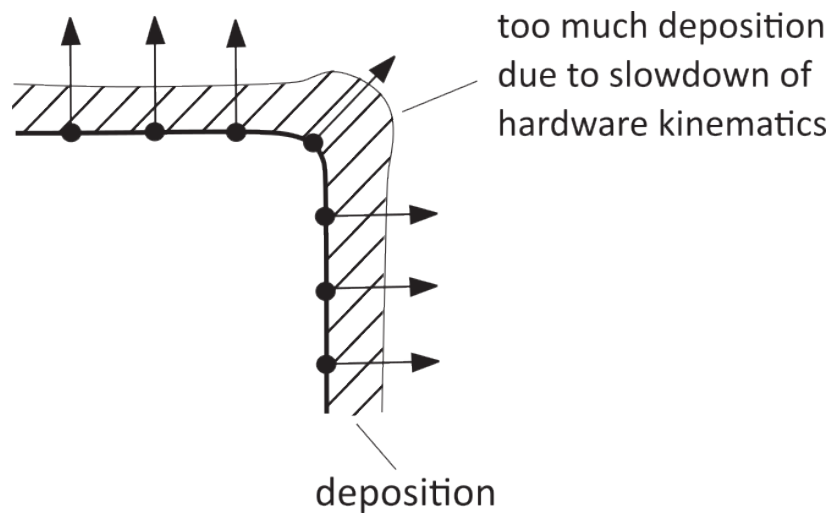

Smoothing reduces the angle change between tool orientations. It is not applied globally, but to groups of sampling points. There are several runs of smoothing. Every run, the group length increases from one to several (three to five) look-ahead distances, which are defined by the user and should cover several sampling points. If all points have the same orientation without collision, it leaves the group untouched. If not, for every group, it calculates the average orientation and adjusts the tool orientation to minimize orientation changes and smooth reversal moves, as shown in Figure 151. There is a rough searching step for every third point and a final search for every point involved. The search consists of rough steps of three degrees and then fine steps of 0.3 degrees. If a common orientation is found, it sets these values and exits the function. If not, it will find subgroups of points with a small variation in values within the group. Averages of the identified subgroups are considered stable values. All orientations of the points between stable subgroups and groups are then interpolated.

Figure 151: Tool orientation before and after smoothing
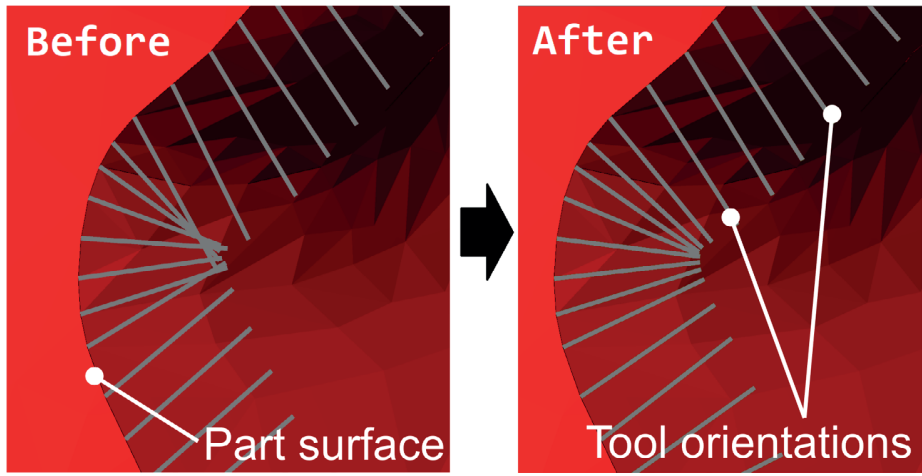
Finally, all moves are continuously checked for dynamic collision, not only at the sample points but at all positions, by simulation tool motion. In case of collision, the orientation is perturbated at just one sampling point without the application of extra smoothing afterward. The algorithm does not allow the maximum lead/lag and tilt angles to be exceeded. If the maximum tilt angle needs to be exceeded in order to avoid a collision, then the toolpath will be split and trimmed, and it will be indicated that a collision will not be avoided. For a better overview of the algorithm, a simplified flowchart is provided in Figure 152.

Figure 152: Workflow of the auto-tilting algorithm

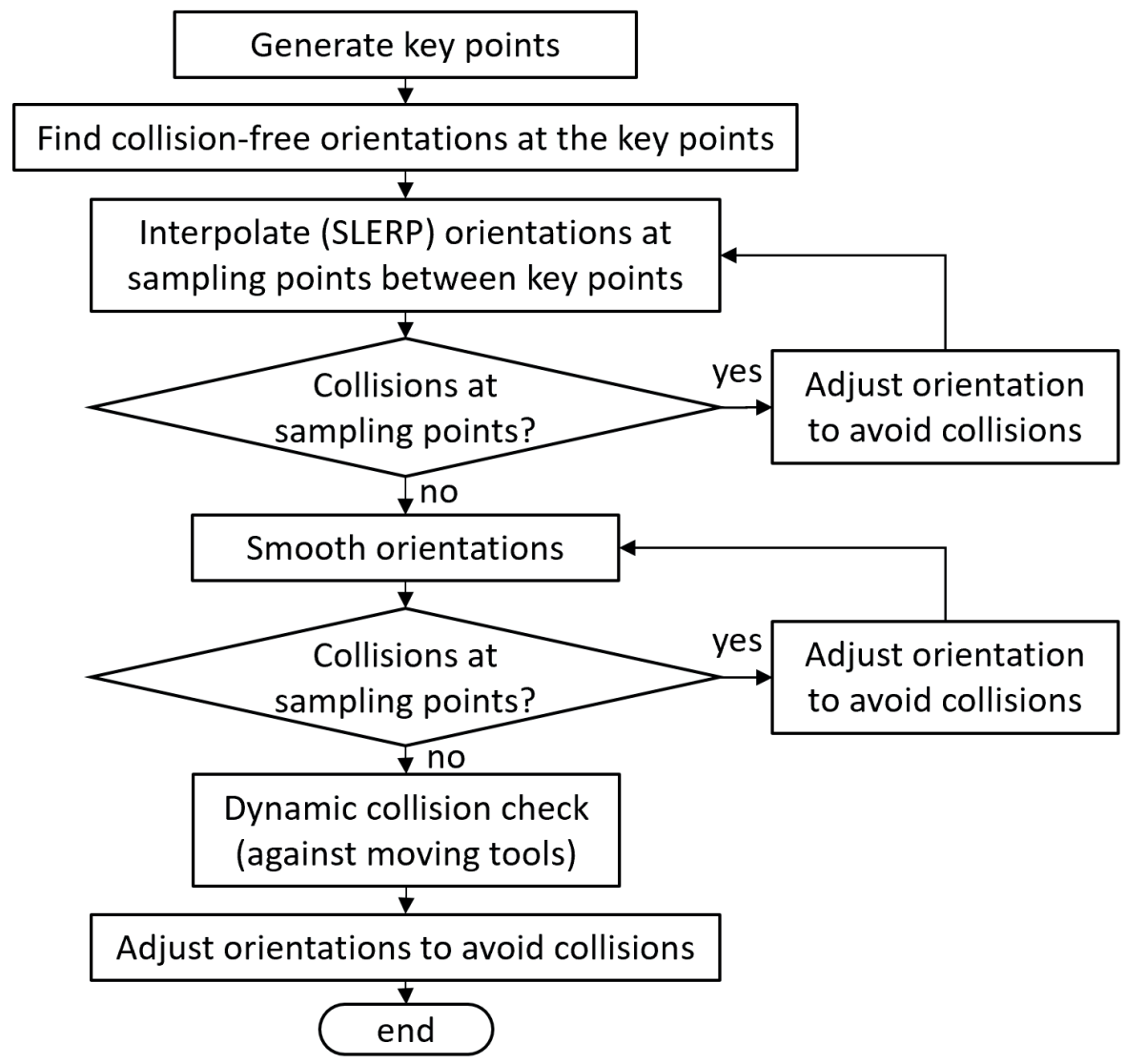




\subsubsection{Experimental validation (single track with smoothing and collision avoidance)}

The experimental validation was tested for 12 double tracks with a stepover of $1.4 \mathrm{~mm}$. The goal of the experiment is to evaluate the effect of smoothing, sampling distance and different strategies (one-way, zigzag, tilting, etc.) on the quality of the weld bead. The quality was assessed visually by observing all build-up formations and irregularities.

\subsubsection{Experimental setup}

The setup configurations are presented in Table 3 and shown in Figure 153. In order to evaluate the influence of collision avoidance, a virtual fixture in the form of a prismatic block has been added to the setup, as shown in Figure 153. This virtual fixture was not present during the actual experiment, as shown in Figure 154.

The parameters in Table 3 combine variation of different tool orientations, different look ahead distances for smoothing and different distance values between sampling points. The algorithm was able to calculate expected tool paths for all these variations by preserving collision freeness. Further research is needed to define best parametrization for different geometries and applications. The goal of this chapter is accomplished by providing an algorithm that accepts user input of relevant parameters and delivering collision-free tool paths compliant with user parameters defining the process.

Figure 153: Geometry of the experimental validation (12 tracks)

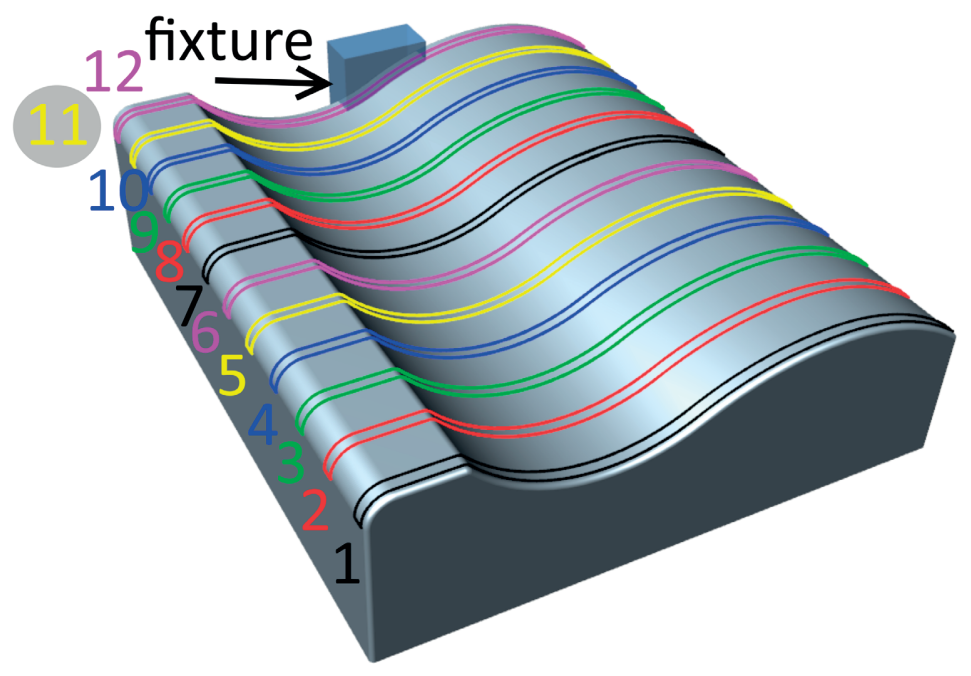


The experiment was carried out on an OKUMA MU 6300V Laser EX machine with the following settings:

- Laser focus with round shape, spot diameter: $2.6 \mathrm{~mm}$;

- Intensity profile: Gaussian;

- Laser power: 990W;

- Feedrate: $530 \mathrm{~mm} / \mathrm{min}$;

- Powder type: LPW H13 Tool steel;

- Powder feed: $6.3 \mathrm{~g} / \mathrm{min}$;

- Shielding gas type: Argon;

- Shielding gas feed: 7 l/min;

- Conveying gas type: Helium;

- Conveying gas feed: $4 \mathrm{l} / \mathrm{min}$.

Table 3: Parameters of the experiment (per track)

\begin{tabular}{llll} 
Track & Strategy & $\begin{array}{l}\text { Look-ahead } \\
\text { distance, } \mathrm{mm}\end{array}$ & $\begin{array}{l}\text { Distance between } \\
\text { sampling points, } \mathrm{mm}\end{array}$ \\
\hline $1^{\star}$ & Three-axis & Disabled & $1-10$ \\
\hline $2^{\star}$ & Five-axis & Disabled & $1-10$ \\
\hline $3^{\star}$ & Five-axis & 10 & $1-10$ \\
\hline $4^{\star}$ & $\begin{array}{l}\text { Five-axis + maximum angle change of 15 degrees } \\
\text { (per move) }\end{array}$ & Disabled & $1-10$ \\
\hline $5^{\star}$ & $\begin{array}{l}\text { Five-axis + maximum angle change of 15 degrees } \\
\text { (per move) }\end{array}$ & 10 & $1-10$ \\
\hline 6 & Five-axis & Disabled & $1-10$ \\
\hline 7 & Five-axis & 10 & $1-10$ \\
\hline 8 & Five-axis & Disabled & $10-100$ \\
\hline 9 & Five-axis & 10 & $10-100$ \\
\hline 10 & Five-axis + tilt angle of 25 degrees & Disabled & $1-10$ \\
\hline 11 & Five-axis + collision & 4 & $1-10$ \\
\hline $12^{\star *}$ & Five-axis + collision & 4 & $1-10$ \\
\hline
\end{tabular}

* Tracks 1-5 are one-way, while tracks 6-12 are zigzag

** The virtual fixture definition is close to track 12 such it imposes a strong tilt to avoid it 
Gigure 154: Machine setup during the experiment

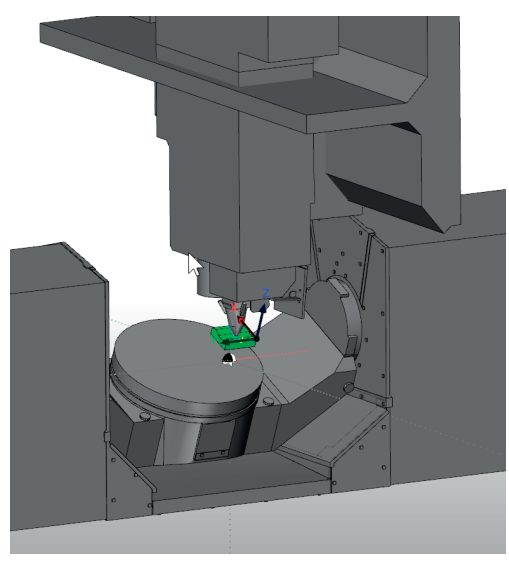

a) in CAM environment

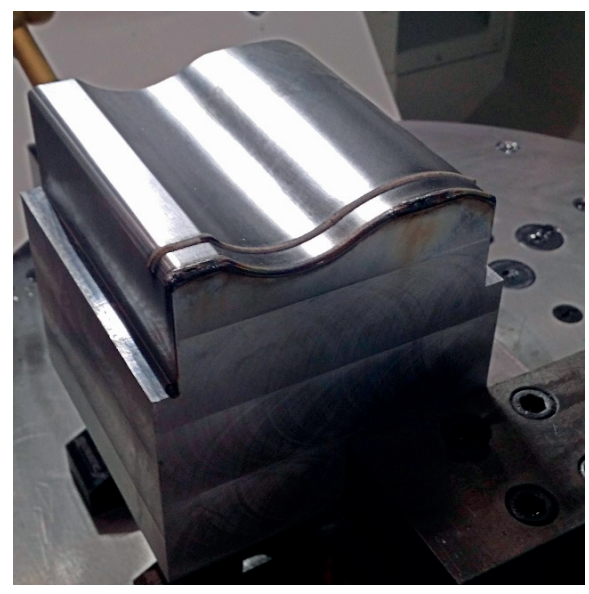

b) Actual setup on the machine

\subsubsection{Machining experiment}

The top view of the deposited tracks is shown in Figure 155. Additionally, several images of the relative nozzle workpiece positions are shown in Figure 156.

Figure 155: Result of the experimental validation (12 tracks)

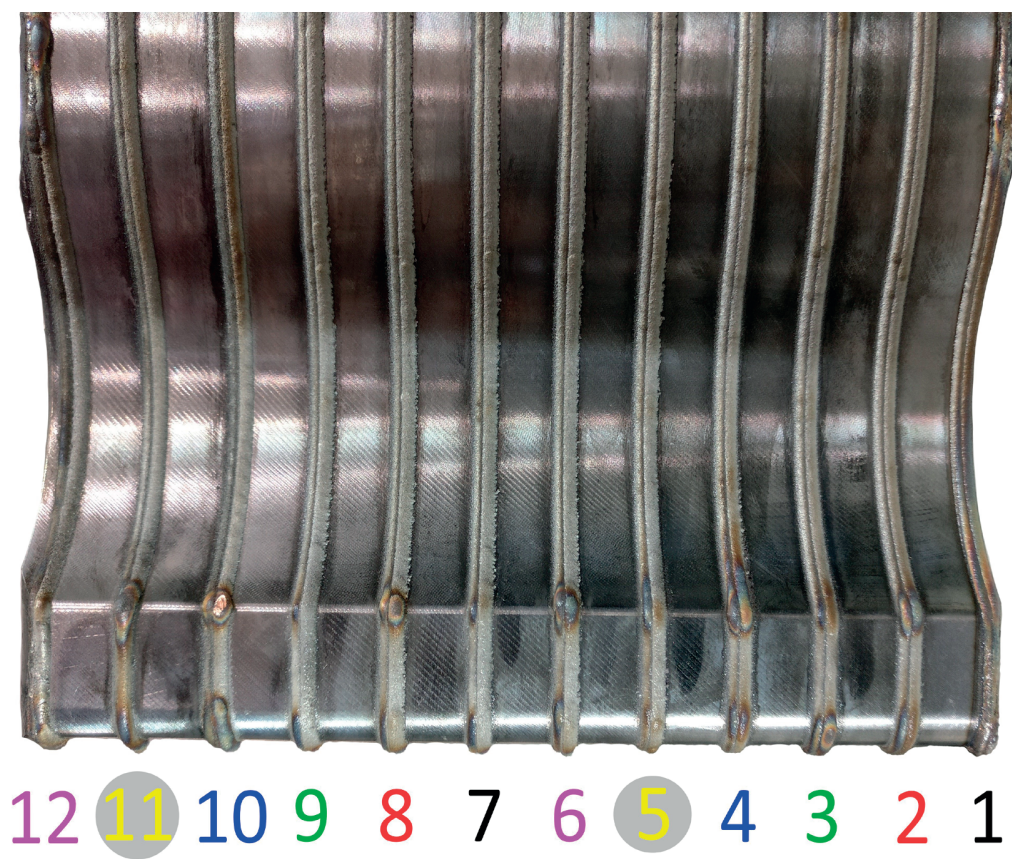


Figure 156: Photos of the part and nozzle during the validation test

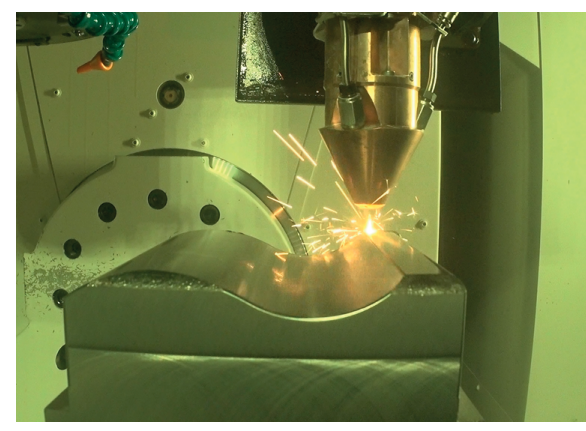

a) Normal to surface, flat region

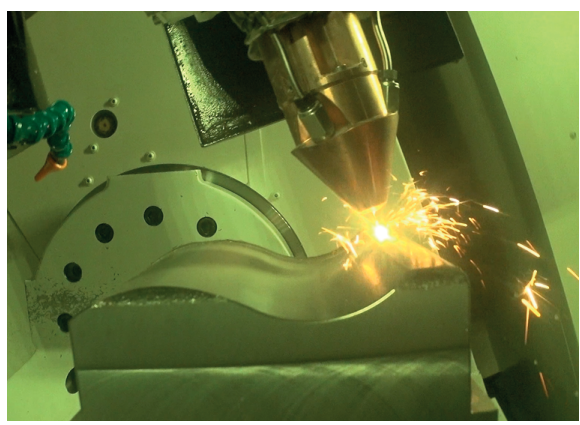

c) Smoothed, flat region

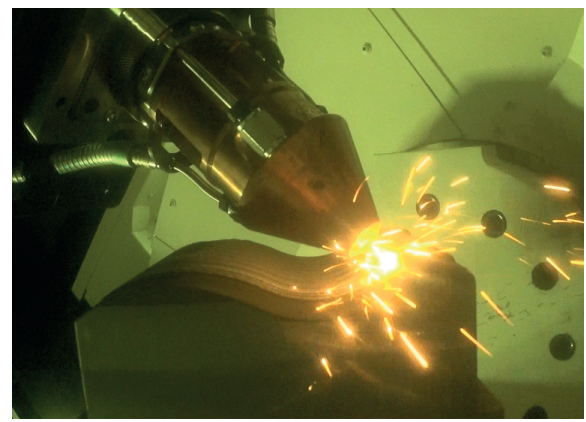

e) Smoothing before avoiding the virtual obstacle

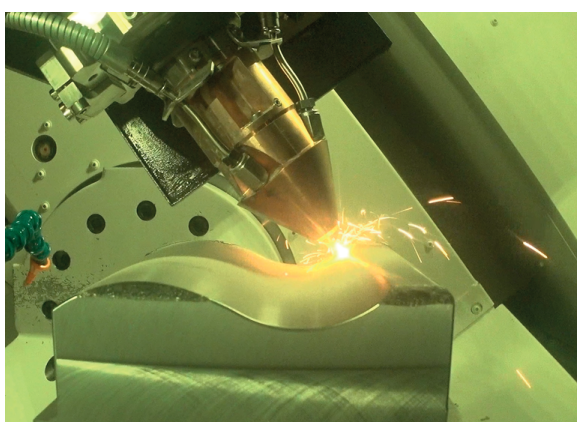

b) Normal to surface, after the edge

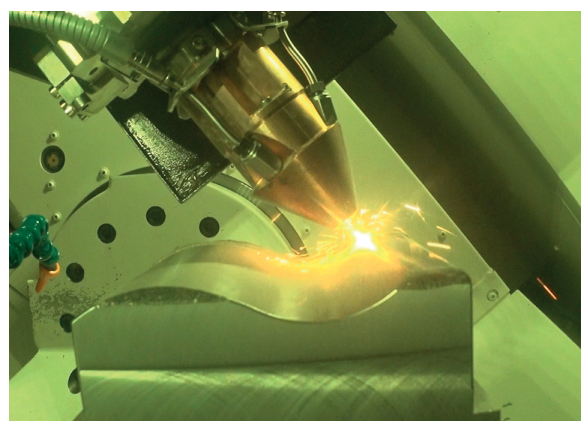

d) Smoothed, after the edge

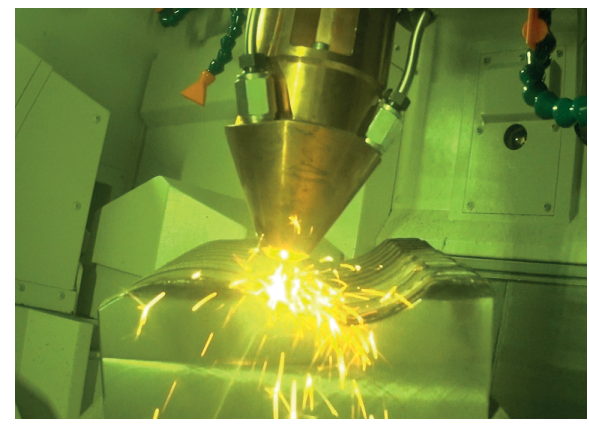

f) Avoiding the virtual obstacle

\subsubsection{Ultrasonic measurements}

The deposited weld beads (tracks) were measured using ultrasonic testing. In ultrasonic testing, low-amplitude waves are propagated through a material to measure either or both the time of travel and any change in intensity over a given distance. Pulse echo principles are used to calculate the ultrasonic reflection or transition time through the body to be measured. When an ultrasonic signal travels through an interface of mismatched impedance that depends on the speed of sound and density, there are reflections at the boundaries between the different 
materials. The echo that is generated by the transducer travels through the components. This is usually refracted at the boundaries between the different materials and reflected on the surface. The reflected ultrasonic waves provide valuable information about the surface or the contact situations. The reflected signals offer useful information about the integrity or the geometry of the object to be tested. The measurement setup is shown in Figure 157.

Figure 157: Ultrasonic test setup

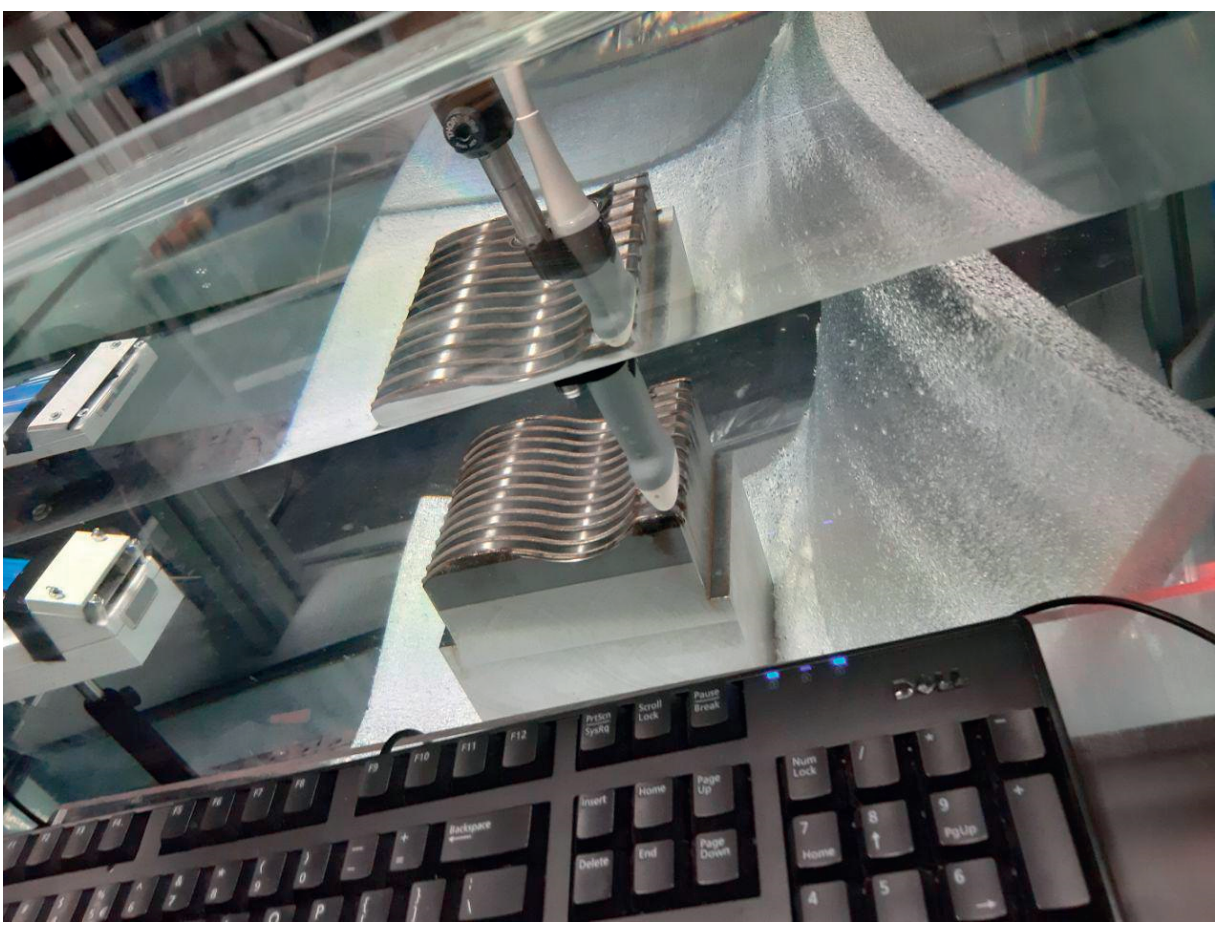

The measurements were performed with the ultrasonic probe SCA02 at a frequency of 14.5 $\mathrm{MHz}$. The axial resolution of a B-mode ultrasound system is half the spatial pulse length, 0.1 microseconds, and the speed of sound in water, which is $\sim 1485 \mathrm{~m} / \mathrm{s}$. Thus, the axial resolution is $148 \mu \mathrm{m}$. The probe specifications are summarized in Figure 158.

The shape of the beads was measured at three locations, as shown in Figure 159. First, the longitudinal profile of the beads on the flat regions (beginning of tracks) was measured. Secondly, the cross-sections of all beads along the edge and curved regions were measured. The ultrasonic images are depicted in black and white, where the white color represents the deposited material, as shown in Figure 160. Images of the beads are presented in Figure 161, Figure 162 and Figure 163. Measurements of the bead widths and heights are presented in Figure 164 and Figure 165. 
Figure 158: Specifications of the ultrasonic probe used

Product Name: PB-SC1-4H: 510K Approved

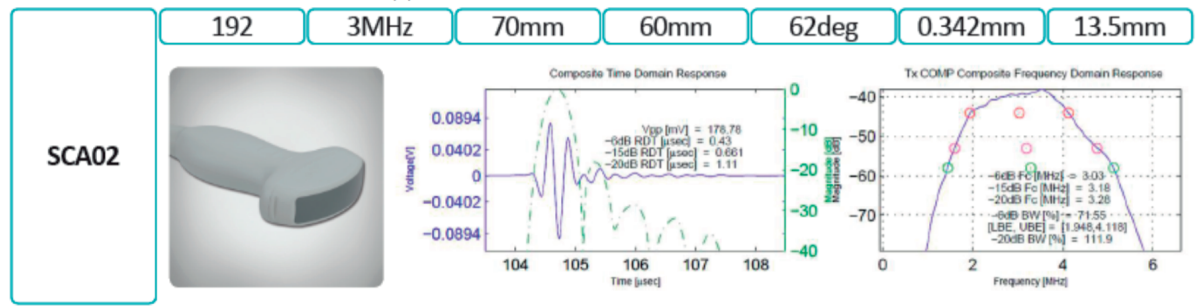

- Figure 159: Measurement plan of 12 tracks. Bead cross-sections at the edge and curved region; longitudinal profile of beads on the flat regions.

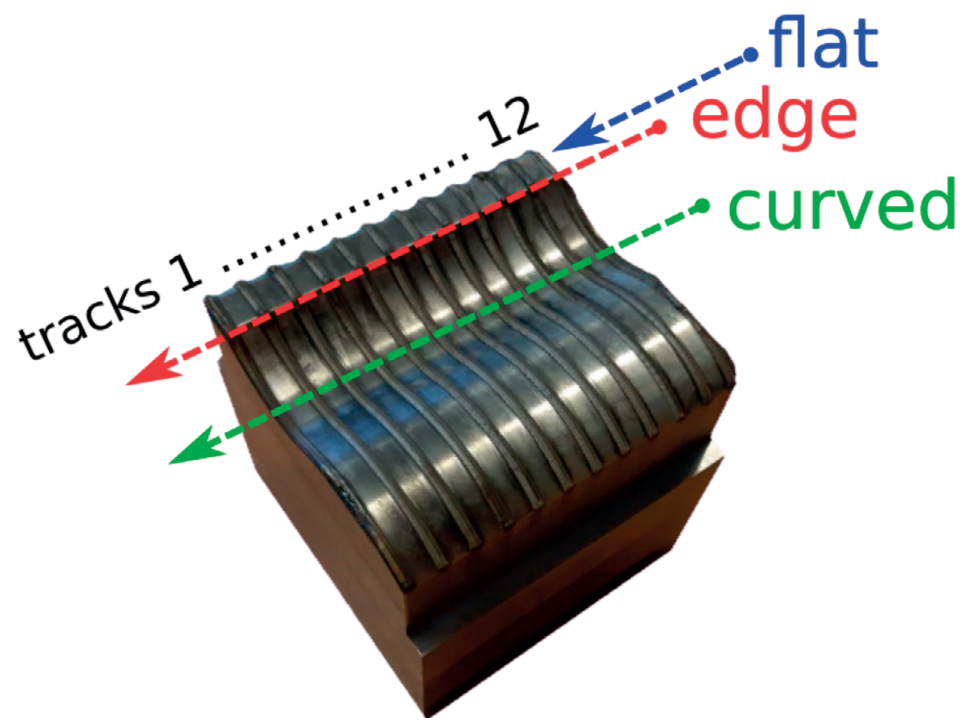

Figure 160: Decoding of the bead image

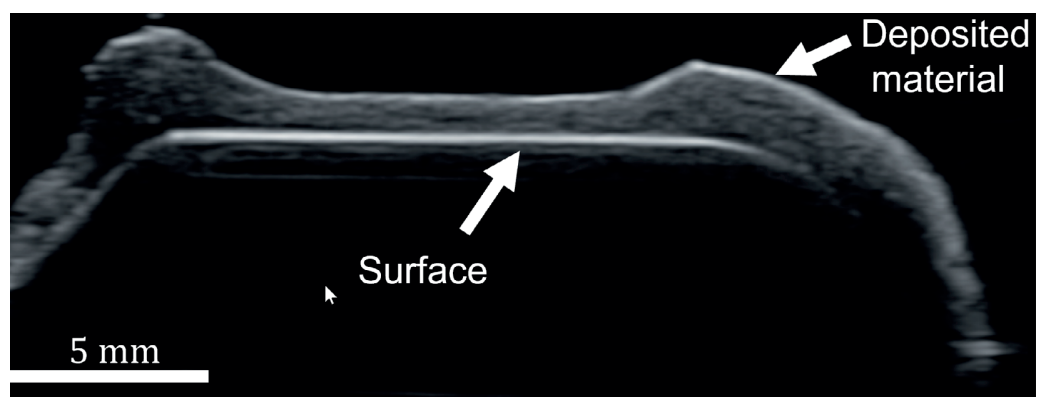


- Figure 161: Longitudinal bead profiles of 12 tracks
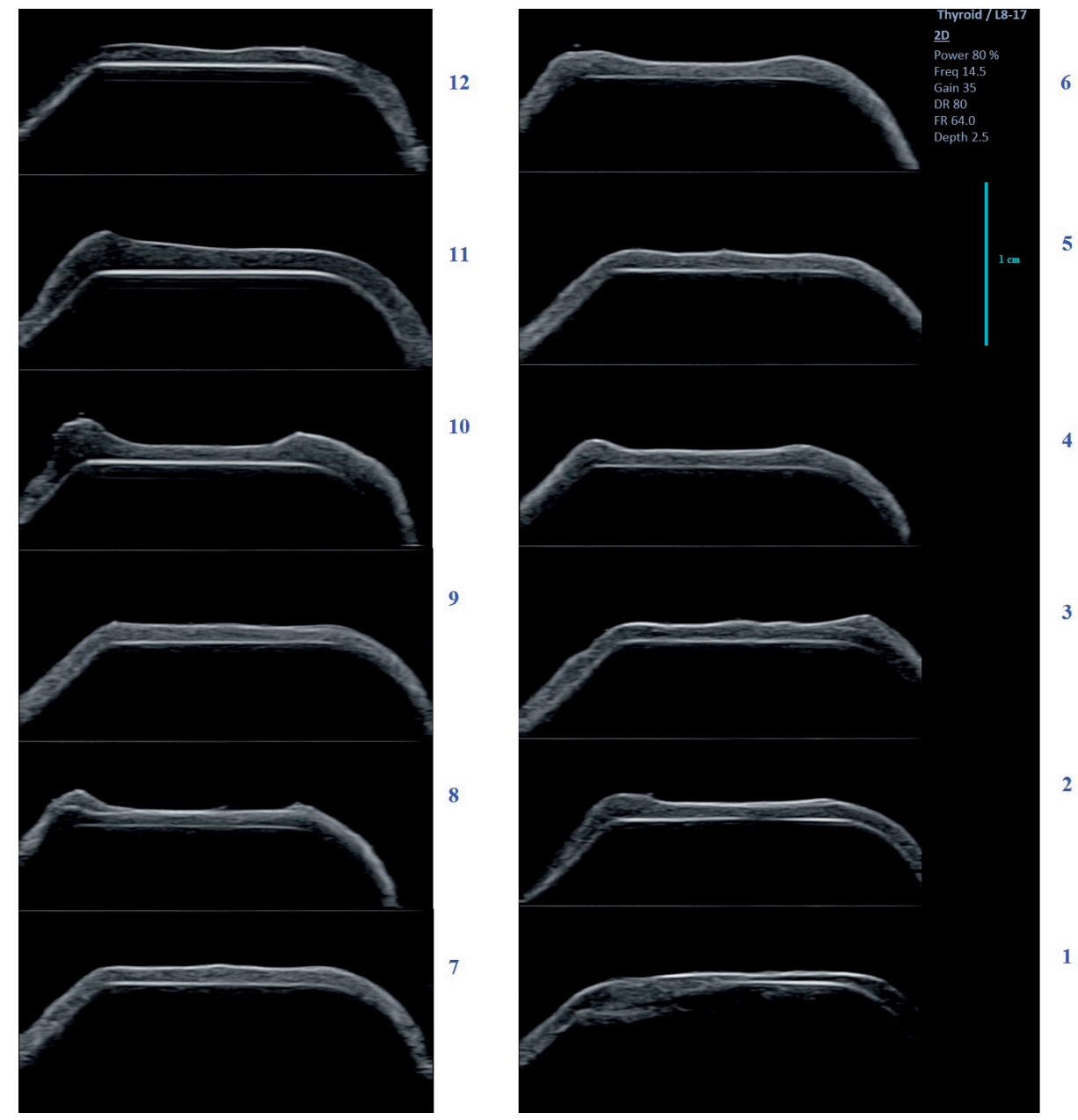
Figure 162: Cross-section of weld bead with various parameters on the edge surfaces of 12 tracks

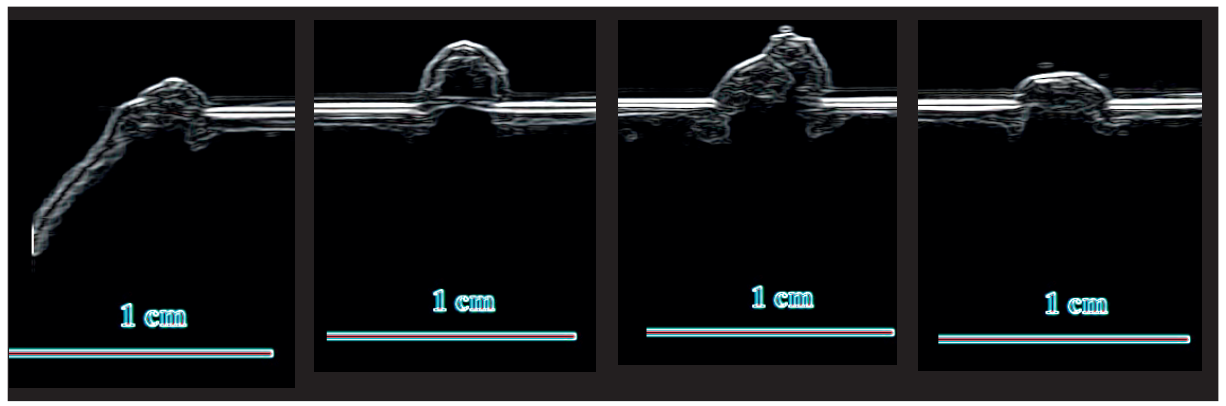

12

11

10

9

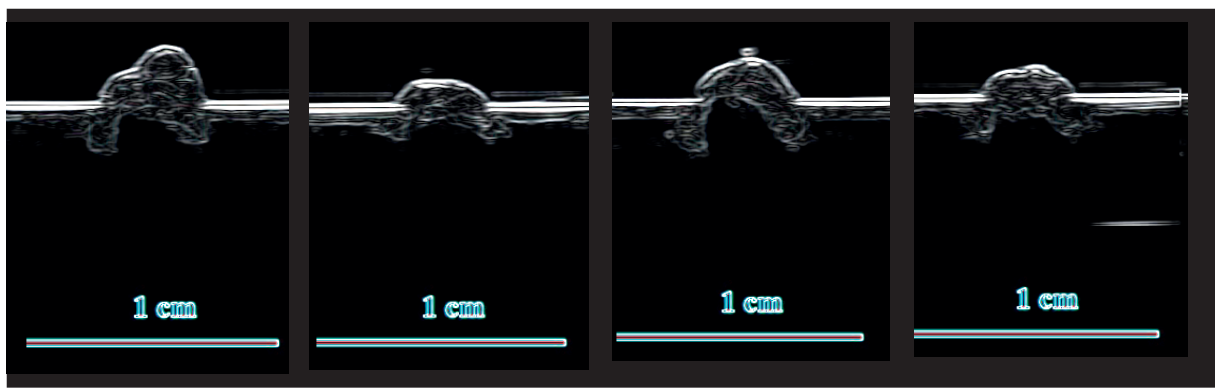

8

7

6

5

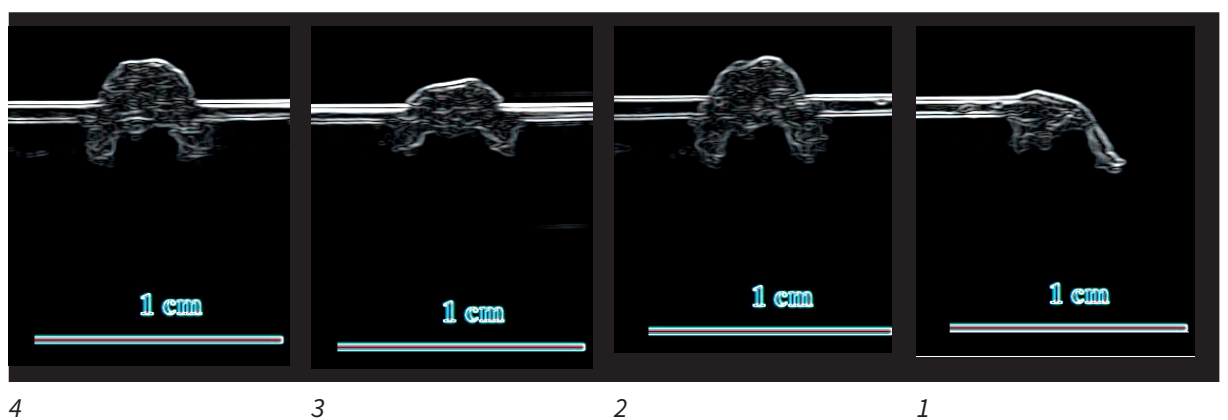


- Figure 163: Cross-section of weld bead with various parameters on curved surfaces for 12 tracks
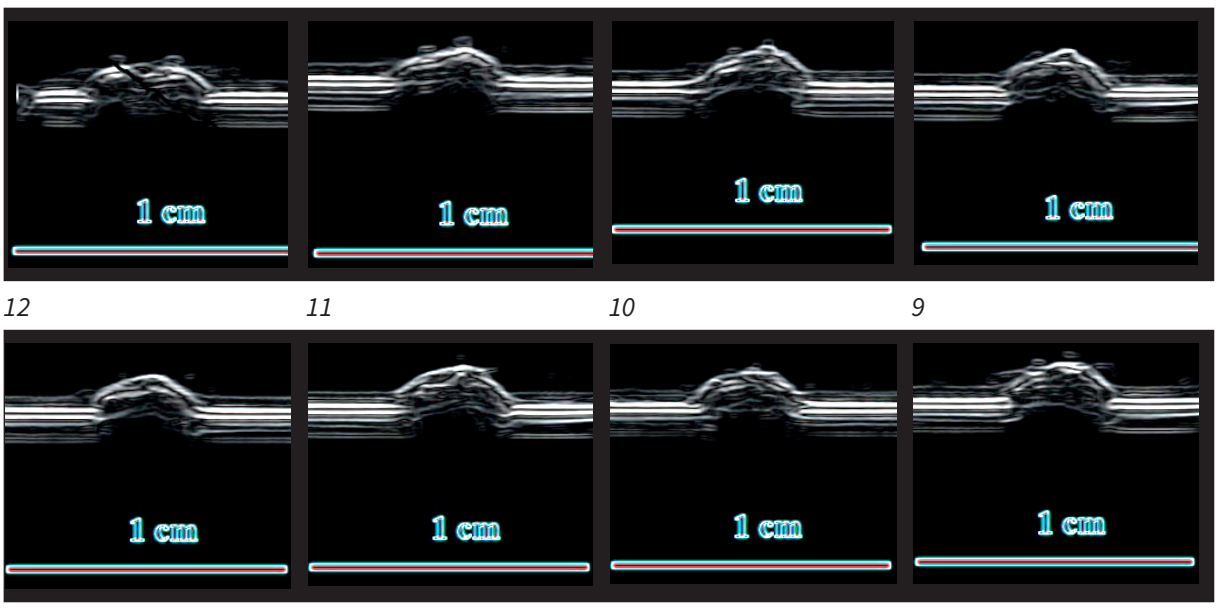

5

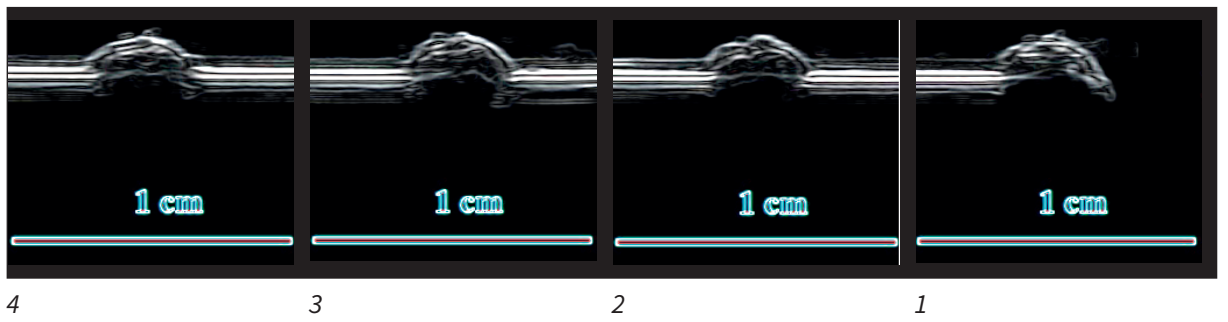


Figure 164: Weld bead height and width on the curved region

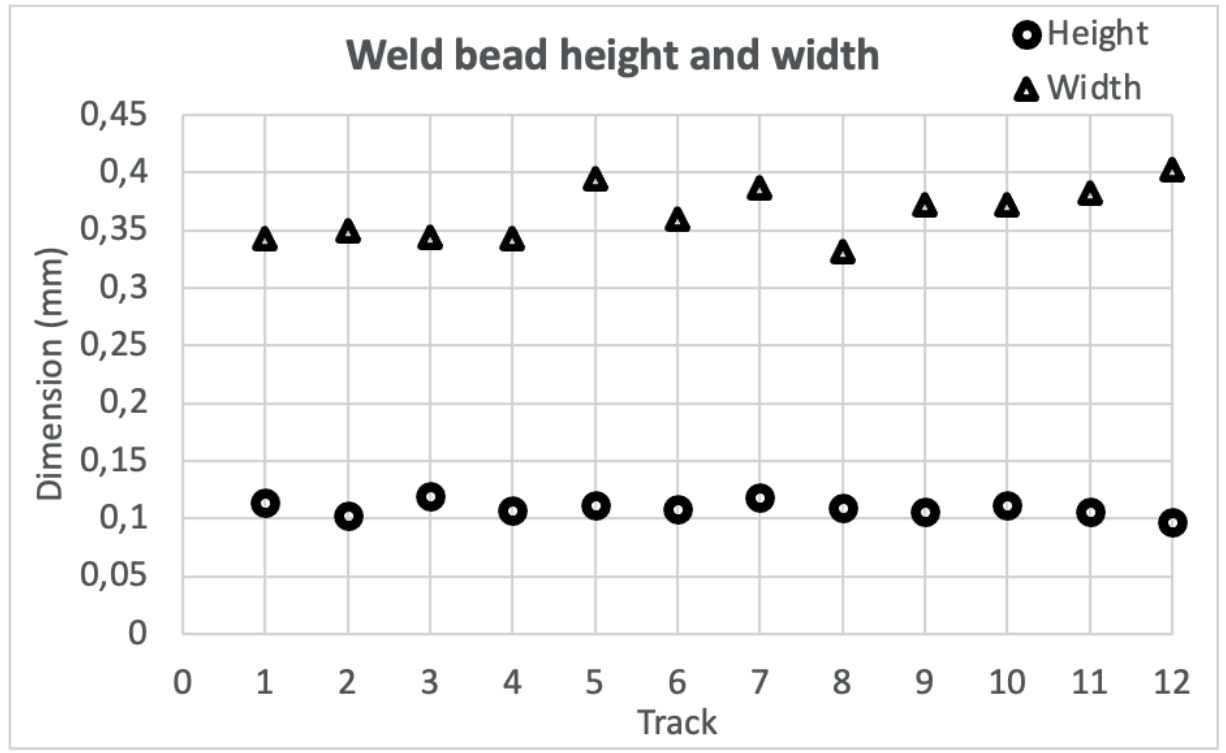

Figure 165: Weld bead height/width ratio on the curved region

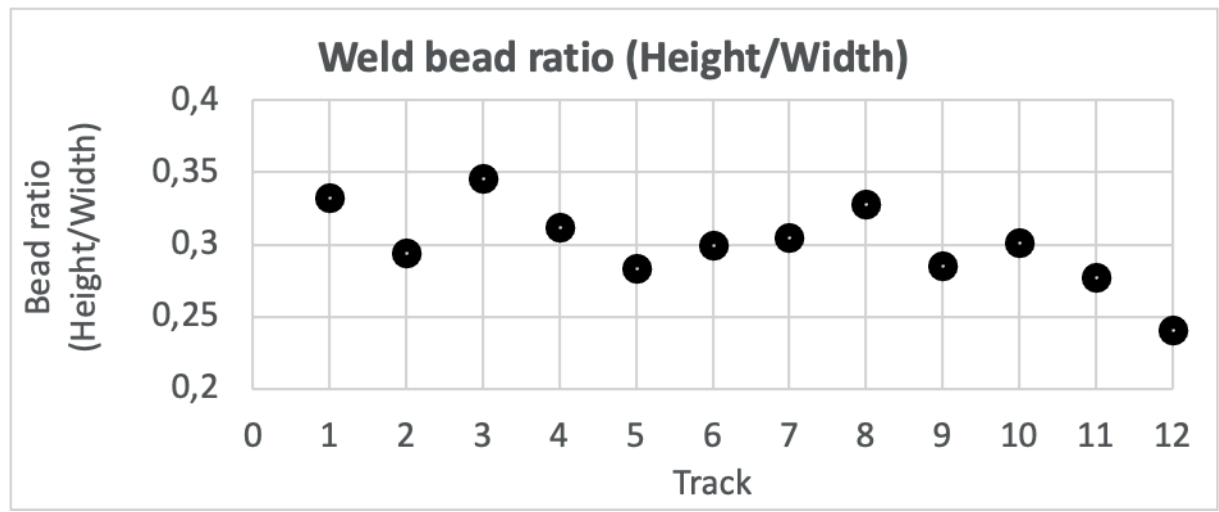




\subsubsection{Analysis of the results and conclusion}

In contrast with conventional machining, the quality of DED-generated surfaces appears to be very sensitive to feed variation during the process. This feed variation may have different sources. Firstly, the machine dynamics may not reach the required levels of angular acceleration and jerk to maintain the programmed feed for moves with large angular changes of the rotary axes. Secondly, sampling of the points of DED toolpaths may influence the CNC of the DED equipment. Sampling points that are too dense are believed to overflow the computational capacity of the controller, which will mean it will not be able to interpolate all the necessary sampling points at the rate of the programmed feed (compare Track 2 and Track 8 in Figure 155).

Smoothing of the tool orientation results in a better quality of the deposited material in regions where there are abrupt changes in the tool orientation (compare Track 2 to Track 3 and Track 9 in Figure 155). Despite smoothing, the weld bead at Track 12 has quality issues due to the tilt angle exceeding an empiric rule-of-thumb limit of 25 degrees. The exact reason for the quality degradation is not known to the authors because bead formation is a complex phenomenon that depends on many factors (reflections, distribution of energy in the laser beam, gas turbulence, etc.).

Observations and short analysis of the different tracks are summarized in Table 4.

Table 4: Observations on the experiment (per track)

\section{Track Observation}

1 This three-axis toolpath has some defects that can be attributed to the tool orientation deviating toward the surface normal.

2 Since the tool follows the surface normal, build-up peaks in areas of higher curvature are expected. The higher heat transfer into the weld bead is confirmed by the bead color.

3 With smoothing, the quality is better than in Track 2.

$4 \quad$ By limiting the maximum angle change, the quality achieved in highly curved paths suffers.

$5 \quad$ The effects of a limited maximum angle change (Track 4) were relaxed by smoothing.

$6 \quad$ This is a zigzag twin of Track 2.

7 This is a zigzag twin of Track 3. The difference is not significant. However, there is likely to be a difference between the zig and zag directions.

8 This is a twin of Track 2 with fewer sampling points. Reduction of sampling points slightly improved the quality.

9 With a greater point distance, the machine runs a little bit jerky, but the influence of the smoothing is stronger, so the material build-up on the sharp corners is minimal.

10 Since the nozzle was tilted by -25 degrees, the weld bead quality is compromised.

11 Since the smoothing and the collision tilting are used in parallel (due to the virtual fixture), they produce large machine motions of the $A$ and $C$ axes, followed by $X, Y$ and $Z$. However, the weld bead has decent quality, with the exception of the sharp transition. That can be explained by the use of a shorter look-ahead distance of $4 \mathrm{~mm}$, which led to less efficient smoothing.

12 Tilting to avoid collision with the virtual fixture has severely affected the quality of the weld bead. 


\subsubsection{Experimental validation (geodesic and offset layers)}

The experimental validation of geodesic toolpaths was tested on the mold part shown in Figure 166a. The toolpath was generated on the part surface with an offset of $2 \mathrm{~mm}$ from the guide curve, as shown in Figure 166b. The toolpath extent was limited to the containment body.

The geometry of the mold part represents a typical level of complexity for a DED repair application. The part has flat and curved surfaces, along with cavities that contribute to the worn areas. To ensure a more homogeneous transition between the base and deposited materials, the metal deposition is not limited to the cavities. Two toolpath layers were generated. The first layer is a geodesic toolpath on the input geometry, as shown in Figure 167a. The second layer is an offset layer with an offset distance of $2 \mathrm{~mm}$. Both layers are shown in Figure 167b.

Next, collision-free and smooth orientation vectors in both layer toolpaths were generated, as shown in Figure 168.

Figure 166: Mold part for testing

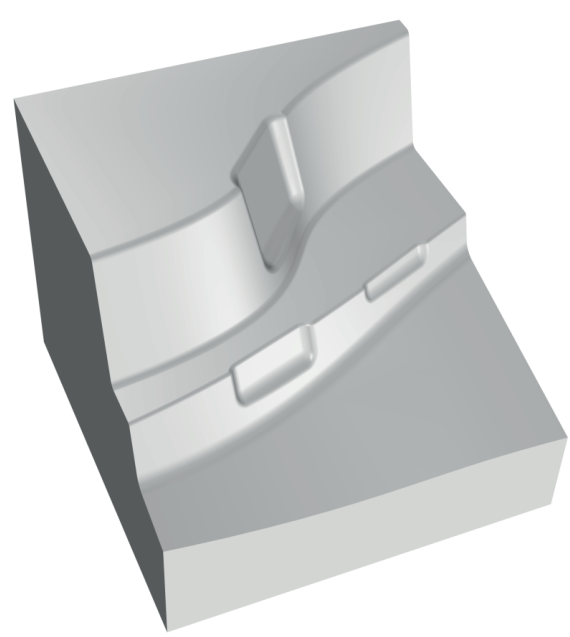

a) Mold part

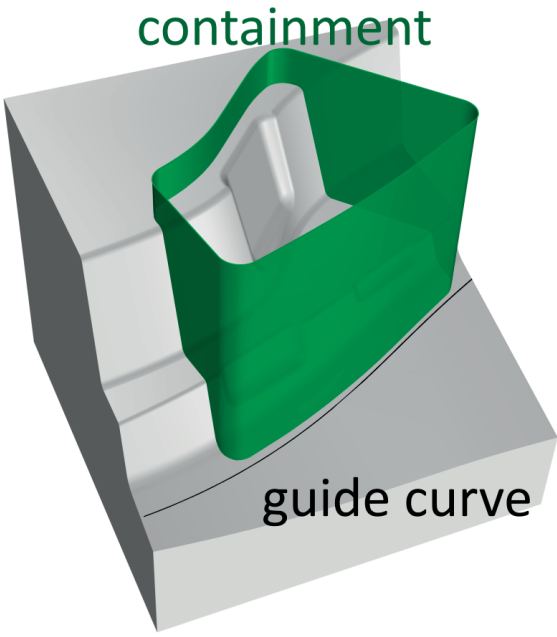

b) Input geometries for geodesic toolpaths 
- Figure 167: Toolpath layers on the mold part

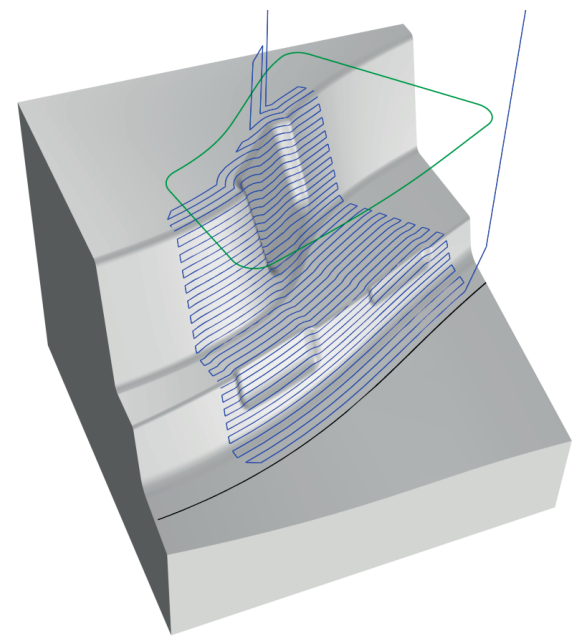

a) First toolpath layer (blue lines)

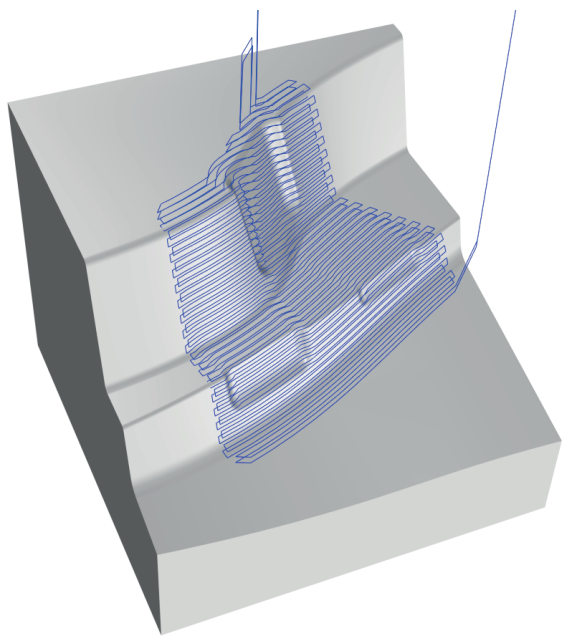

b) First and second toolpath layer (blue lines)

Figure 168: Collision-free orientations on the mold part

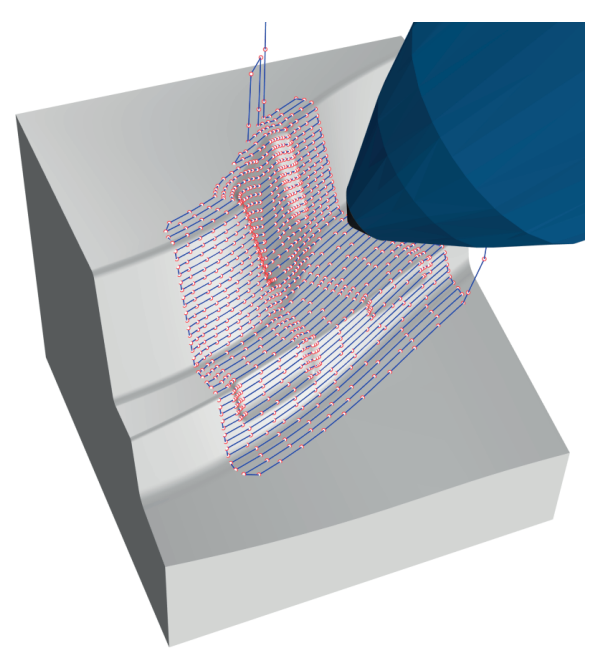

a) Point on the first layer toolpath (blue lines) where collisions are computed

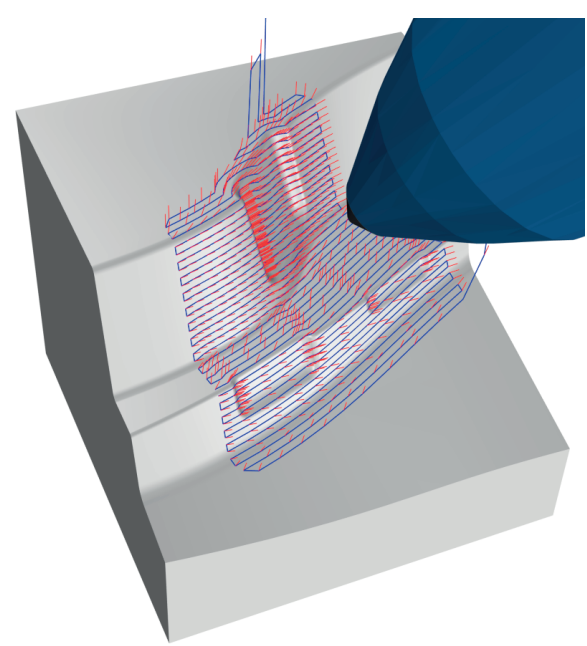

b) First toolpath layers (blue lines) with nozzle orientations (red lines) 


\subsubsection{Experimental setup}

The experiment was carried out on an OKUMA MU 6300V Laser EX machine. The experiment started with the mold part, as shown in Figure 169. Figure 170 shows the part with the first layer deposited. Figure 171 to Figure 173 show images taken at different stages for the second layer. Finally, Figure 174 depicts the part after deposition. Visually, the deposited surface is uniform and does not have significant irregularities. The cusp height variation seems to be acceptable since further machining is required anyway. Additionally, the nozzle did not collide with the part. An example of this collision avoidance can be seen in Figure 172, where the nozzle is inclined outward from the vertical sidewall to avoid collision.

Figure 169: Experimental setup of geodesic multi-layer toolpaths before the deposition

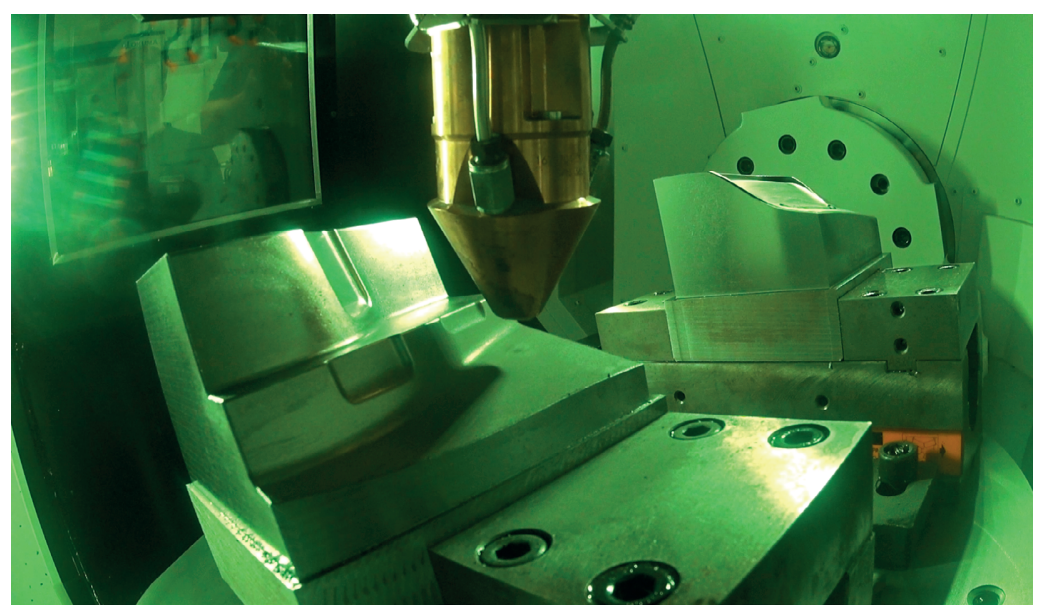

Figure 170: Experimental setup of geodesic multi-layer toolpaths after the first layer

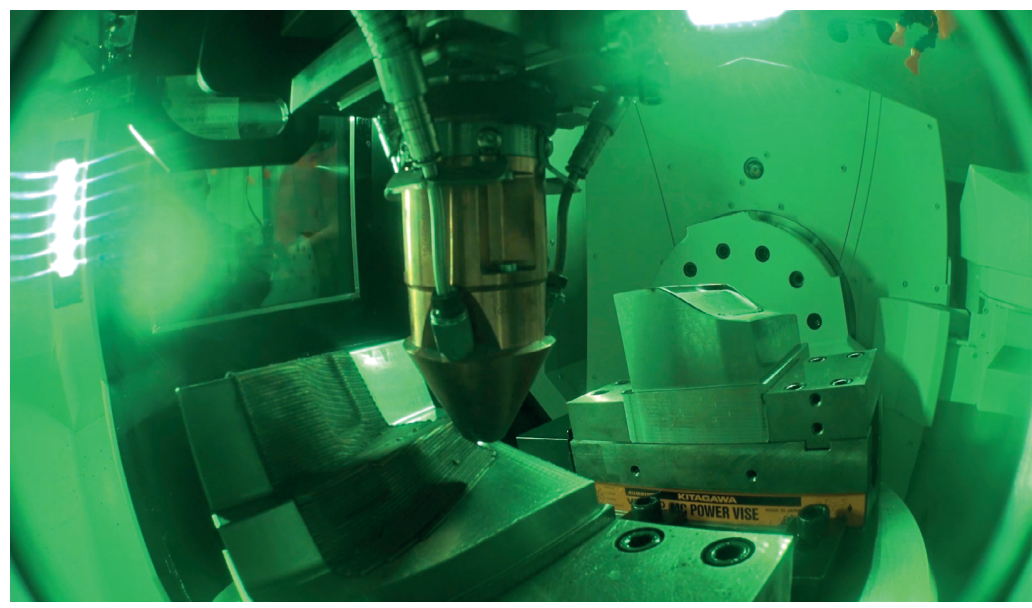


- Figure 171: Experimental setup of geodesic multi-layer toolpaths, beginning of the second layer

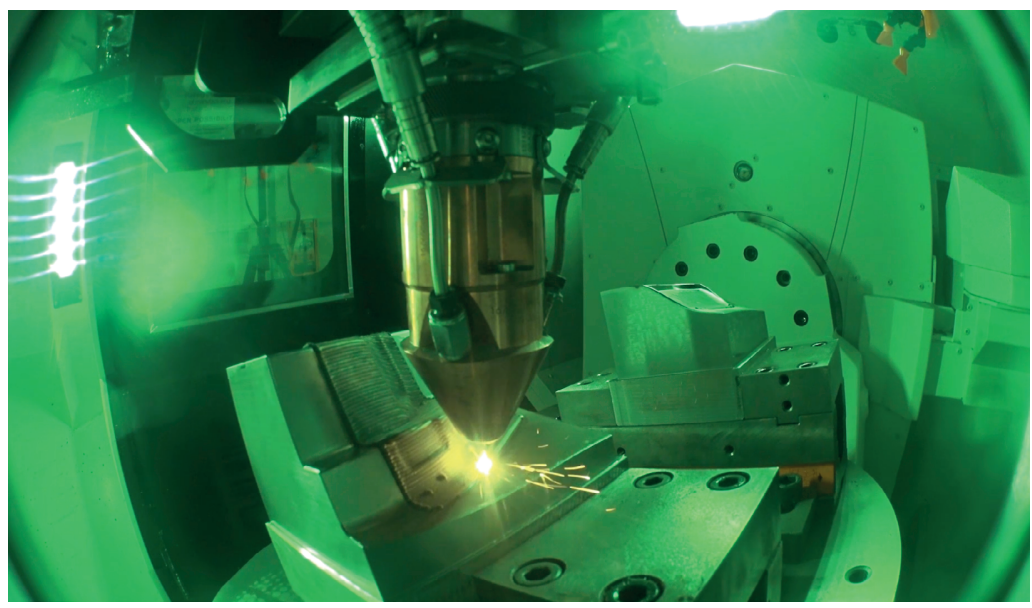

Figure 172: Experimental setup of geodesic multi-layer toolpaths, middle of the second layer

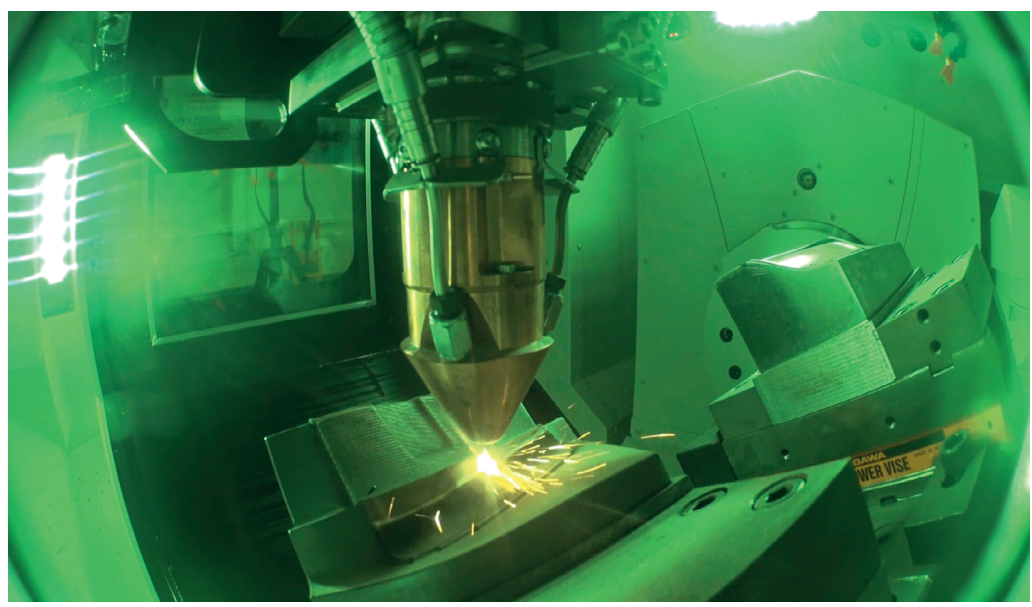


- Figure 173: Experimental setup of geodesic multi-layer toolpaths, end of the second layer

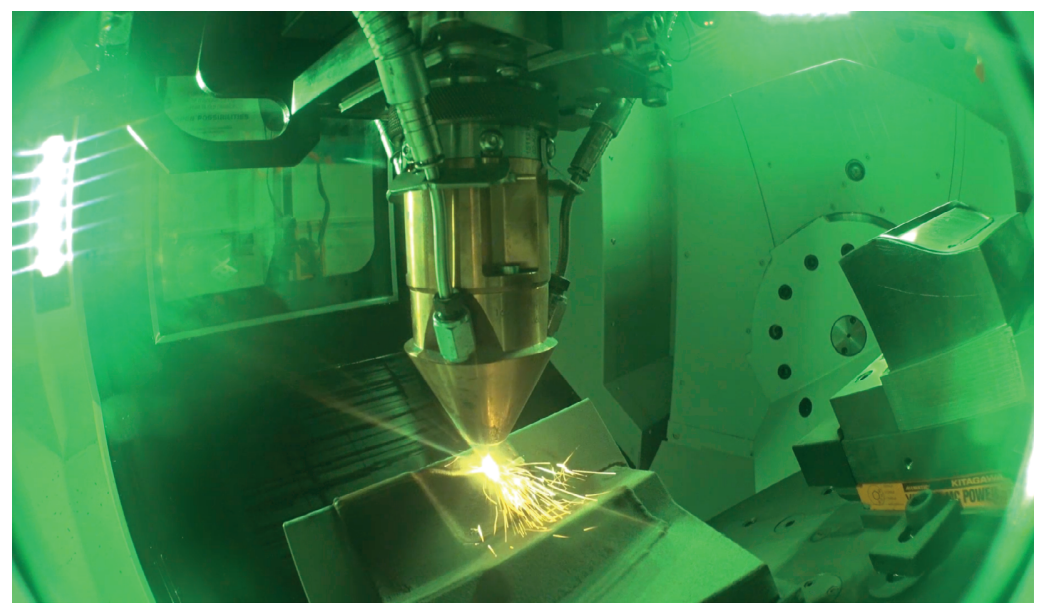

Figure 174: Mold part with two geodesic layers deposited

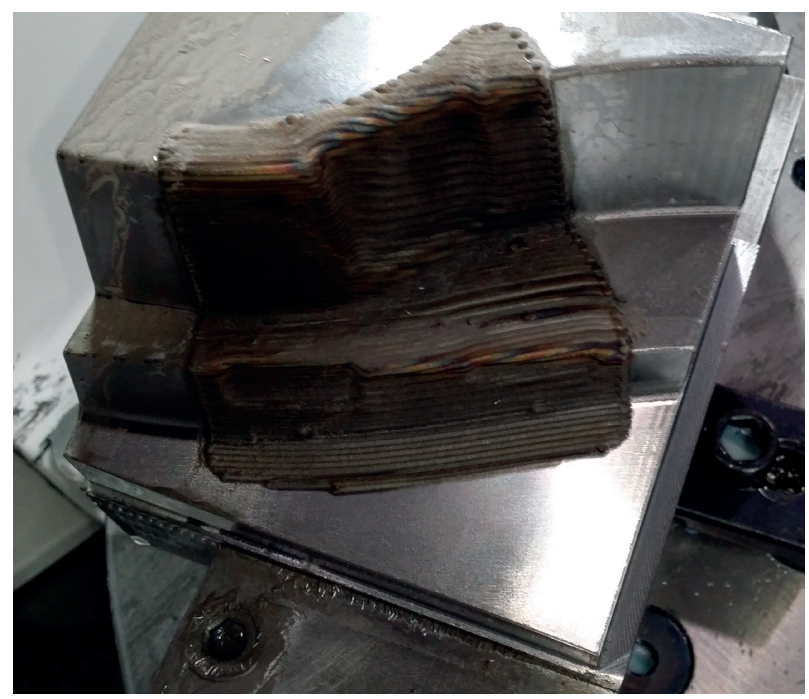




\subsection{CONCLUSION}

The foundation of the presented strategy is the combination of automatic tool orientation smoothing and collision avoidance. Important parameters are the lead/lag angles, side tilt angles, sampling distance between toolpath points, look-ahead distance and full collision avoidance. As such, different variations of these parameters are applied to the test part and calculations are performed. The resulting tool paths are tested on the machine and differences in results are observed as described above. Further research on the impact of these parameters for different applications can be conducted in the future using the developed algorithm.

The main benefits of this algorithm can be concluded as following:

- Collision-free multi-axis AM and smooth tool paths are calculated

- Significant process related parameters can be provided by the user like tilt angle along path

- Tool path calculation time is optimized due to optimized algorithm for tilting (within minutes versus within hours)

- The algorithm can work with any triangle mesh as geometry input since the offset approach is forgiving any disjoints between triangles

- The distance between neighbor deposition paths can be calculated exactly due to exact geodesic algorithm used on triangle meshes

- The algorithm is a part geometry and machine kinematic agnostic solution, such it allows further researchers to use this as a basis to conduct process research on multi-axis AM

As a final conclusion, it will be checked if the research questions from Chapter 1 have been addressed. What are the answers to these questions?

Research question: Can novel toolpath strategies for DED be developed that result in smooth deposition along 3D curves?

This chapter presents a novel toolpath strategy for DED, which has been implemented and validated with machining trials. The underlying generic geometrical data model and the generic algorithm have proven capable of handling the test geometry and provide the process planner with many variation possibilities for the process parameters, like the deposition nozzle orientation in relation to surface normal and move direction. Thus, the research question can be answered positively. The generic algorithmic model and the rich variety of process planning parametrization provides future researchers a strong basis to work on improvements for different aspects of the AM process. Such this research question can be answered positively.

Research question: To what extent modifications of existing subtractive path planning strategies and related algorithms can be used for multi-axis AM? 
The algorithmic approach in this chapter is based on modifications to existing milling strategies. In five-axis milling, the tool direction is often initially calculated based on a surface normal direction with variations of tilting in relation to this normal direction and cut direction. The geometrical computation requirement for collision control is the same for both milling and multi-axis AM. The further requirement for neighboring toolpath contours to be within a defined distance is similar in both cases. However, due to the deposition process, the accuracy of the distance between contours is more critical in AM. This means that developing an exact geodesic toolpath algorithm is necessary, whereas in milling, approximative algorithms are acceptable. 

CHAPTER 5

I Conclusions and outlook 


\subsection{CONCLUSIONS}

In this work, several algorithmic advances in automation of AM process planning have been achieved. The main focus has been on AM processes that selectively deposit material. These processes include FDM and DED, which are gaining momentum in industrial applications because they make it much easier to fabricate functional parts than conventional technologies. One of the biggest drawbacks of these technologies is that support structures are required. The need for support removal is a barrier to adoption because it can be very complicated for some materials, like PEEK or some metals, or not economically viable if the material used is expensive, like titanium alloys.

The developed 3+2 decomposition algorithms (Chapter 3) not only overcomes the problem of support removal but also improve the surface quality. A new metric to show the distribution of the cusp height can be used for efficient analysis of the surface quality. Variation of the algorithm parameters allows the metrics to be progressively improved.

The continuous 5 axis AM differs greatly from traditional layer-by-layer deposition using 3 axis or $3+2$ axis. Additional degrees of freedom create new challenges. The nozzle may collide or produce uneven deposition tracks. Chapter 4 successfully resolves this challenge. The algorithm's settings for smoothing the nozzle orientation vector can be further analyzed to predict the surface quality.

Both methods (decomposition and true multi-axis toolpath generation) complement each other in terms of applications and process requirements. For example, continuous 5 axis AM algorithm can be utilized for repair applications with DED where $3+2$ axis decomposition algorithm can cover support free creation of FDM parts. Each algorithm has its own strengths and use cases. The experience with 5-axis milling tool path algorithms shows, that a set of algorithms are available to CAM software users for programming, such there is no single algorithm covering all the different needs of various applications.

At the end of Chapter 3 and 4, the research questions from Chapter 1 are answered in detail and positively.

\subsection{OUTLOOK}

Was this work able to overcome some of the limitations of state-of-the-art approaches and what remaining limitations are still present?

Some of the major limitations described in section 1.5.3, listed as challenges in multi-axis AM, has been successfully addressed in this work, and the software implementation of the presented ideas was able to prove, in the test cases, that this work can be considered a contribution to the state of the art. However, some further work still needs to be done in the following areas: 
- The $3+2$ algorithm implemented in this work in Chapter 3 has a limitation when dealing with branching shapes, like the letter "T." The presented algorithm will choose one of the possible branches to conclude the next $3+2$ axis orientation for deposition. Some further work needs to be done to extend the algorithm so that after deciding on a branch, the algorithm will come back to the branching position and process the remaining branch or branches. This improvement will allow the algorithm to manufacture more complex shapes, including branching geometries, with the 3+2 AM algorithm. Some preliminary experiments in this direction have been conducted and are presented below in Figure 175. It is expected that, in the future, the $3+2 \mathrm{AM}$ algorithm will be further extended to deal with all shapes of arbitrary complexity.

- Figure 175: An example of 3+2 printing of branching geometries (numbers show the sequence and the arrows show the directions of the build).

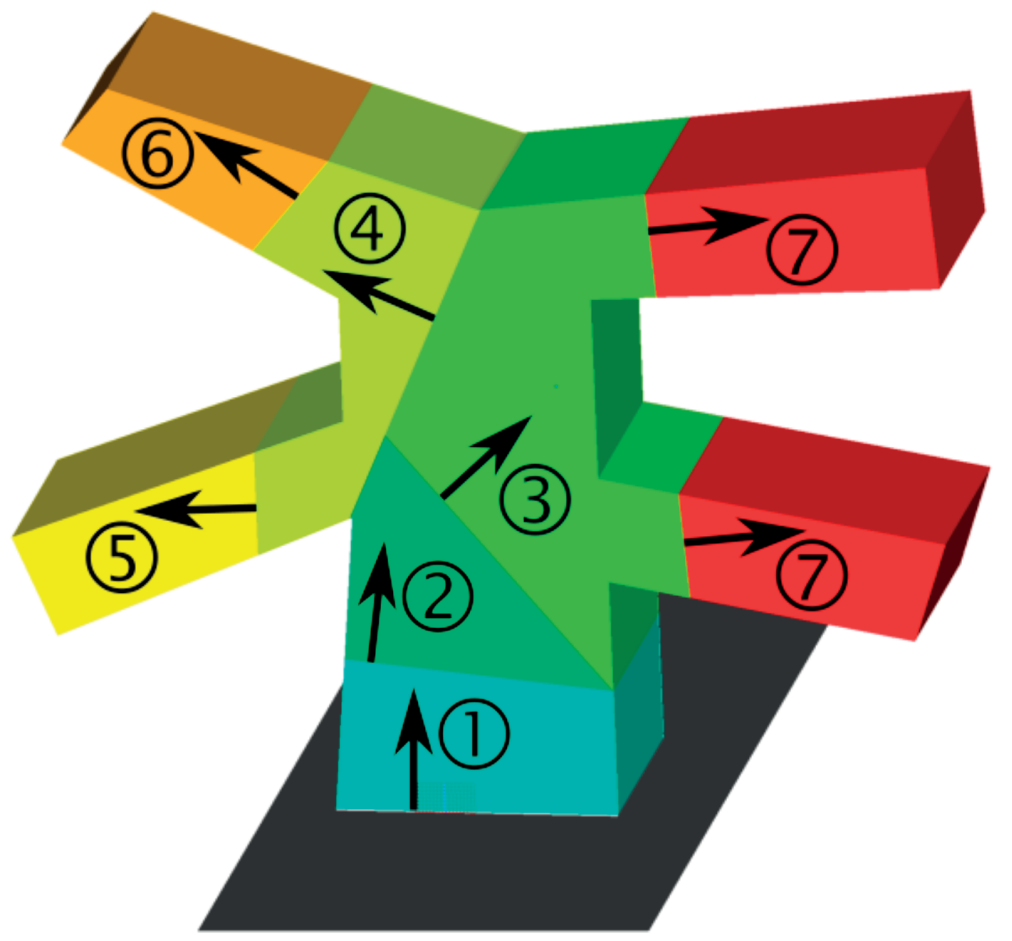

- The $3+2$ algorithm presented in Chapter 3 ensures collision-free deposition of material by using a half-space defined by the nozzle position and its orientation for testing possible collisions against the in-process part shape. Using the real nozzle geometry instead of a half-space will provide a bigger solution set such more complex shapes can be calculated 
successfully without collision. A prototype for this new approach has been implemented as well.

- The experiment presented in Chapter 3 shows further process-related issues, which need to be addressed by improvements in hardware and software. For example, the transition from one partition to the next partition, the visible marks might have an impact not on the visual quality, but also on the static stiffness of the part.

- The experiment presented in Chapter 4 shows that the algorithm provides control on important process parameters and different results can be achieved by this. Detailed research about the process relevant implications of DED process needs to be conducted to deliver more predictable results for various applications.

- The additional freedom through multi-axis AM might have an impact on material properties as a result of more uniform deposition parallel to the part shape. This requires further analysis as well.

Considering the time frame where 5-axis milling became a widely utilized technology was about three decades, it is a legitimate question, how much time it will take for multi-axis AM to become a widely utilized technology. 


\section{BIBLIOGRAPHY}

3D Hubs. (2018). What is 3D printing? The definitive guide. Retrieved from https://www.3dhubs.com/ guides/3d-printing/

3D Platform. (2018). Soluble Support Structures. Common Support Issues in Large Format 3D Printing and How to Fix Them. Retrieved from https://3dplatform.com/soluble-support-structures/

Additiveworks. (n.d.). Overview. Retrieved 04 19, 2020, from https://additive.works/overview

Alexander, P., Allen, S., \& Dutta, D. (1998, 4). Part orientation and build cost determination in layered manufacturing. Computer-Aided Design, 30, 343-356. doi:10.1016/s0010-4485(97)00083-3

Alimardani, M., Toyserkani, E., \& Huissoon, J. P. (2007, 12). A 3D dynamic numerical approach for temperature and thermal stress distributions in multilayer laser solid freeform fabrication process. Optics and Lasers in Engineering, 45, 1115-1130. doi:10.1016/j.optlaseng.2007.06.010

Alimardani, M., Toyserkani, E., Huissoon, J. P., \& Paul, C. P. $(2009$, 11). On the delamination and crack formation in a thin wall fabricated using laser solid freeform fabrication process: An experimental-numerical investigation. Optics and Lasers in Engineering, 47, 1160-1168. doi:10.1016/j.optlaseng.2009.06.010

Bagg, S. D., Sochalski-Kolbus, L. M., \& Bunn, J. R. (2016, 6 29). The effect of laser scan strategy on distortion and residual stresses of arches made with selective laser melting. Retrieved from https://ntrs.nasa. gov/archive/nasa/casi.ntrs.nasa.gov/20160009706.pdf

Baumers, M., Tuck, C., \& Hague, R. (2015). Selective heat sintering versus laser sintering: comparison of deposition rate, process energy consumption and cost performance. Solid Freeform Fabrication Symposium.

Benouamer, M. O., \& Michelucci, D. (1997). Bridging the gap between CSG and Brep via a triple ray representation. Proceedings of the fourth ACM symposium on Solid modeling and applications - SMA 97. ACM Press. doi:10.1145/267734.267755

Bertoldi, M., Yardimci, M., Pistor, C., \& Guceri, S. I. (1998). Domain Decomposition and Space Filling Curves in Toolpath Planning and Generation.

Boisselier, D., Sankar_, S., \& Engel, T. (2014, 1). Improvement of the Laser Direct Metal Deposition Process in 5-axis Configuration. Physics Procedia, 56, 239-249. Retrieved from http://www.sciencedirect. com/science/article/pii/S1875389214003137

Bommes, D., \& Kobbelt, L. (2007). Accurate Computation of Geodesic Distance Fields for Polygonal Curves on Triangle Meshes. VMV.

Boris. (2018). Marching Cubes Tutorial. Retrieved 12 21, 2019, from https://www.boristhebrave. com/2018/04/15/marching-cubes-tutorial/

Bournias-Varotsis, A., Wang, S., Hutt, D., \& Engstrøm, D. S. (2018, 3). The Effect of Ultrasonic Additive Manufacturing on Integrated Printed Electronic Conductors. Electronic Materials Letters, 14, 413-425. doi:10.1007/s13391-018-0053-y

Breen, D. E., \& Mauch, S. (1999). Generating Shaded Offset Surfaces with Distance, Closest-Point and Color Volumes. In Proceedings of the International Workshop on Volume Graphics, (pp. 307-320). 
Buchbinder, D., Meiners, W., Pirch, N., Wissenbach, K., \& Schrage, J. (2014, 2). Investigation on reducing distortion by preheating during manufacture of aluminum components using selective laser melting. Journal of Laser Applications, 26, 012004. doi:10.2351/1.4828755

Bunch, B. H. (2004). The History of Science and Technology: A Browser's Guide to the Great Discoveries, Inventions, and the People Who Made Them from the Dawn of Time to Today. Houghton Mifflin Harcourt. Retrieved from https://www.amazon.com/History-Science-Technology-Discoveries-Inventions/ $\mathrm{dp} / 0618221239$ ?SubscriptionId=AKIAIOBINVZYXZQZ2U3A\&tag=chimbori05-20\&linkCode=xm2\&camp $=2025 \&$ creative $=165953 \&$ creativeASIN $=0618221239$

Cain, P. (2019). Selecting the optimal shell and infill parameters for FDM 3D Printing. Retrieved 1029,2019 , from https://www.3dhubs.com/knowledge-base/selecting-optimal-shell-and-infill-parameters-fdm-3d-printing/

Cain, V., Thijs, L., Humbeeck, J. V., Hooreweder, B. V., \& Knutsen, R. (2015, 1). Crack propagation and fracture toughness of Ti6Al4V alloy produced by selective laser melting. Additive Manufacturing, 5, 68-76. doi:10.1016/j.addma.2014.12.006

Campocasso, S., Chalvin, M., Reichler, A.-K., Gerbers, R., Droder, K., Hugel, V., \& Dietrich, F. (2018). A framework for future CAM software dedicated to additive manufacturing by multi-axis deposition. Procedia CIRP, 78, 79-84. doi:10.1016/j.procir.2018.08.314

Cao, J., Gharghouri, M. A., \& Nash, P. $(2016,11)$. Finite-element analysis and experimental validation of thermal residual stress and distortion in electron beam additive manufactured Ti-6Al-4V build plates. Journal of Materials Processing Technology, 237, 409-419. doi:10.1016/j.jmatprotec.2016.06.032

Cesarano, J. (1998). A Review of Robocasting Technology. MRS Proceedings, 542. doi:10.1557/proc-542-133

Chakraborty, D., Aneesh Reddy, B., \& Roy Choudhury, A. (2008, 2). Extruder path generation for Curved Layer Fused Deposition Modeling. Computer-Aided Design, 40, 235-243. Retrieved from http://www. sciencedirect.com/science/article/pii/S0010448507002412

Chalcraft, E. (2013, 5 17). Mataerial by Petr Novikov, Saša Jokić, Joris Laarman Lab and IAAC. Retrieved from https://www.dezeen.com/2013/05/17/mataerial-3d-printer-by-petr-novikov-sasa-jokic-and-joris-laarman-studio/

Chalvin, M., Campocasso, S., Baizeau, T., \& Hugel, V. (2019). Automatic multi-axis path planning for thinwall tubing through robotized wire deposition. Procedia CIRP, 79, 89-94. doi:10.1016/j.procir.2019.02.017

Chen, Y., Zhou, C., \& Lao, J. (2013, 5). A layerless additive manufacturing process based on CNC accumulation. Rapid Prototyping Journal, 17, 218-227. doi:10.1108/13552541111124806

Chengkai Dai, C. W., \& Liu, Y.-j. (2018, 8). Support-Free Volume Printing by Multi-Axis Motion. ACM Trans. Graph., 37, 13. doi:10.1145/3197517.3201342

Chiu, W. K., Yeung, Y. C., \& Yu, K. M. (2006, 8). Toolpath generation for layer manufacturing of fractal objects. Rapid Prototyping Journal, 12, 214-221. doi:10.1108/13552540610682723

Clarke, C. (2017). Fabrisonic granted ultrasonic additive manufacturing patent. Retrieved 06 03, 2020, from https://3dprintingindustry.com/news/fabrisonic-granted-ultrasonic-additive-manufacturing-patent-106376/

Coupek, D., Friedrich, J., Battran, D., \& Riedel, O. (2018, 1). Reduction of Support Structures and Building Time by Optimized Path Planning Algorithms in Multi-axis Additive Manufacturing. Procedia CIRP, 67, 221-226. Retrieved from http://www.sciencedirect.com/science/article/pii/S2212827117311472 
Crump. (1989). USA Patent No. US5121329A.

Daumas, M. (1970, 5). Progress through the Ages in A History of Technology and Invention; Translated from the French edition (Paris, 1962 and 1964) by Eileen B. Hennessy. Science, 1, The Origins of Technological Civilization, 596. Retrieved from http://science.sciencemag.org/content/168/3932/726.2.abstract

Ding, D., Pan, Z., Cuiuri, D., \& Li, H. (2014, 4). A tool-path generation strategy for wire and arc additive manufacturing. The International Journal of Advanced Manufacturing Technology, 73, 173-183. doi:10.1007/s00170-014-5808-5

Ding, D., Pan, Z., Cuiuri, D., \& Li, H. (2015, 8). A practical path planning methodology for wire and arc additive manufacturing of thin-walled structures. Robotics and Computer-Integrated Manufacturing, 34, 8-19. doi:10.1016/j.rcim.2015.01.003

Ding, D., Pan, Z., Cuiuri, D., Li, H., Larkin, N., \& Duin, S. (2016, 2). Automatic multi-direction slicing algorithms for wire based additive manufacturing. Robotics and Computer-Integrated Manufacturing, 37, 139-150. Retrieved from http://www.sciencedirect.com/science/article/pii/S0736584515000873

Dolenc, A., \& Mäkelä, I. (1994, 2). Slicing procedures for layered manufacturing techniques. Computer-Aided Design, 26, 119-126. doi:10.1016/0010-4485(94)90032-9

Dwivedi, R., \& Kovacevic, R. (2004, 1). Automated torch path planning using polygon subdivision for solid freeform fabrication based on welding. Journal of Manufacturing Systems, 23, 278-291. doi:10.1016/ s0278-6125(04)80040-2

Dwivedi, R., \& Kovacevic, R. (2005, 7). Process Planning for Multi-Directional Laser-Based Direct Metal Deposition. Proceedings of the Institution of Mechanical Engineers, Part C: Journal of Mechanical Engineering Science, 219, 695-707. doi:10.1243/095440605×31535

Dwivedi, R., \& Kovacevic, R. (2006, 11). An expert system for generation of machine inputs for laser-based multi-directional metal deposition. International Journal of Machine Tools and Manufacture, 46, 1811-1822. doi:10.1016/j.ijmachtools.2005.11.008

Dwivedi, R., \& Kovacevic, R. $(2007,6)$. A novel approach to fabricate uni-directional and branching slender structures using laser-based direct metal deposition. International Journal of Machine Tools and Manufacture, 47, 1246-1256. doi:10.1016/j.ijmachtools.2006.08.012

Eckes, K. (2018). The wasteful truth about industrial plastics 3D printing. Retrieved May 24, 2020, from https://aerosint.com/the-wasteful-truth-about-industrial-plastics-3d-printing/

Elliott, A. (2019). Advancing Liquid Metal Jet Printing. Tech. rep., Oak Ridge National Lab.(ORNL), Oak Ridge, TN (United States).

Fadhli. (2019). 3d printing hearing aid industry. Retrieved 8 2, 2019, from https://3dprintingindustry. co/3d-printing-hearing-aid-industry/

Farouki, R. T., Koenig, T., Tarabanis, K. A., Korein, J. U., \& Batchelder, J. S. (1995). Path planning with offset curves for layered fabrication processes. Journal of Manufacturing Systems, 14, 355-368. doi:10.1016/0278-6125(95)98872-4

Feng, H.-Y., \& Li, H. (2002, 8). Constant scallop-height tool path generation for three-axis sculptured surface machining. Computer-Aided Design, 34, 647-654. doi:10.1016/s0010-4485(01)00136-1

Ford, S., \& Despeisse, M. (2016, 11). Additive manufacturing and sustainability: an exploratory study of the advantages and challenges. Journal of Cleaner Production, 137, 1573-1587. doi:10.1016/j. jclepro.2016.04.150 
Foteinopoulos, P., Papacharalampopoulos, A., \& Stavropoulos, P. (2018, 1). On thermal modeling of Additive Manufacturing processes. CIRP Journal of Manufacturing Science and Technology, 20, 66-83. doi:10.1016/j.cirpj.2017.09.007

Frikel, G. $(2020,08)$. Development of an optimized path planning method for robot-assisted 3D printing. Master Thesis, TH Köln. Deutschland.

Fuchs, H., Kedem, Z. M., \& Naylor, B. F. $(1980,7)$. On visible surface generation by a priori tree structures. ACM SIGGRAPH Computer Graphics, 14, 124-133. doi:10.1145/965105.807481

Gao, W., Zhang, Y., Nazzetta, D. C., Ramani, K., \& Cipra, R. J. (2015). RevoMaker: Enabling Multi-directional and Functionally-embedded 3D printing using a Rotational Cuboidal Platform. Proceedings of the 28th Annual ACM Symposium on User Interface Software \&\#38; Technology (pp. 437-446). Charlotte: ACM. doi:10.1145/2807442.2807476

Gao, W., Zhang, Y., Nazzetta, D. C., Ramani, K., \& Cipra, R. J. (2015). RevoMaker: Enabling Multi-directional and Functionally-embedded 3D printing using a Rotational Cuboidal Platform. Proceedings of the 28th Annual ACM Symposium on User Interface Software \&\#38; Technology (pp. 437-446). Charlotte: ACM. doi:10.1145/2807442.2807476

GE Additive. (2018). New manufacturing milestone: 30,000 additive fuel nozzles. Retrieved August 2019, 10, from https://www.ge.com/additive/blog/new-manufacturing-milestone-30000-additive-fuel-nozzles

Gibson, I., Rosen, D., \& Stucker, B. (2015). Directed Energy Deposition Processes. In Additive Manufacturing Technologies (pp. 245-268). Springer New York. doi:10.1007/978-1-4939-2113-3_10

Gouge, M., \& Michaleris, P. (Eds.). (2017, 8 7). Thermo-Mechanical Modeling of Additive Manufacturing. Elsevier - Health Sciences Division. Retrieved from https://www.ebook.de/de/product/29583432/ thermo_mechanical_modeling_of_additive_manufacturing.html

Groover, M. P. (2013). Fundamentals of modern manufacturing: materials, processes, and systems. Hoboken, NJ: John Wiley \& Sons, Inc.

GSMA. (2019). The Mobile Economy 2019. Retrieved from https://www.gsma.com/r/mobileeconomy/

Guthe, M., Borodin, P., \& Klein, R. $(2005,1)$. Fast and Accurate Hausdorff Distance Calculation between Meshes., 13, pp. 41-48.

Hague, R., Campbell, I., \& Dickens, P. $(2003,1)$. Implications on design of rapid manufacturing. Proceedings of the Institution of Mechanical Engineers, Part C: Journal of Mechanical Engineering Science, 217, 25-30. doi:10.1243/095440603762554587

Haq, R. H., Marwah, O. M., Rahman, M. N., Haw, H. F., Abdullah, H., \& Ahmad, S. (2019, 8). 3D Printer parameters analysis for PCL/PLA filament wire using Design of Experiment (DOE). IOP Conference Series: Materials Science and Engineering, 607, 012001. doi:10.1088/1757-899x/607/1/012001

Harmand, S., Lewis, J. E., Feibel, C. S., Lepre, C. J., Prat, S., Lenoble, A., . . Roche, H. (2015, 5). 3.3-millionyear-old stone tools from Lomekwi 3, West Turkana, Kenya. Nature, 521, 310-315. Retrieved from http://dx.doi.org/10.1038/nature14464

Harvard Graduate School of Design. (2016). Interactive 3D Ceramic Printing: Sydney RobArch Workshop 2016. Retrieved from https://research.gsd.harvard.edu/maps/portfolio/interactive-3d-ceramic-printing-sydney-robarch-workshop-2016/ 
Hauth, S., Murtezaoglu, Y., \& Linsen, L. $(2009,12)$. Extended linked voxel structure for point-to-mesh distance computation and its application to NC collision detection. Computer-Aided Design, 41, 896-906. doi:10.1016/j.cad.2009.06.007

Hauth, S., Richterich, C., Glasmacher, L., \& Linsen, L. (2010, 7). Constant cusp toolpath generation in configuration space based on offset curves. The International Journal of Advanced Manufacturing Technology, 53, 325-338. doi:10.1007/s00170-010-2817-x

He, S., Zeng, X., Yan, C., Gong, H., \& Lee, C.-H. (2017). Tri-Dexel Model Based Geometric Simulation of Multi-axis Additive Manufacturing. In Intelligent Robotics and Applications (pp. 819-830). Springer International Publishing. doi:10.1007/978-3-319-65298-6_73

Holst, A. (2018, 12 11). Global smartphone shipments forecast from 2010 to 2022 (in million units). Retrieved from https://www.statista.com/statistics/263441/global-smartphone-shipments-forecast/

Huang, S. H., Liu, P., Mokasdar, A., \& Hou, L. $(2012,10)$. Additive manufacturing and its societal impact: a literature review. The International Journal of Advanced Manufacturing Technology, 67, 1191-1203. doi:10.1007/s00170-012-4558-5

InssTek. (2016). Application. Retrieved 05 02, 2020, from http://www.insstek.com/content/application

Isa, M. A., \& Lazoglu, I. $(2019,1)$. Five-axis additive manufacturing of freeform models through buildup of transition layers. Journal of Manufacturing Systems, 50, 69-80. doi:10.1016/j.jmsy.2018.12.002

Jee, H., \& Kim, M. (2017, 12). Spherically curved layer (SCL) model for metal 3-D printing of overhangs. Journal of Mechanical Science and Technology, 31, 5729-5735. Retrieved from https://doi.org/10.1007/ s12206-017-1114-0

Jiang, J., Stringer, J., Xu, X., \& Zhong, R. Y. (2018, 4). Investigation of printable threshold overhang angle in extrusion-based additive manufacturing for reducing support waste. International Journal of Computer Integrated Manufacturing, 31, 961-969. doi:10.1080/0951192x.2018.1466398

Jiang, J., Xu, X., \& Stringer, J. (2018, 9). Support Structures for Additive Manufacturing: A Review. Journal of Manufacturing and Materials Processing, 2, 64. doi:10.3390/jmmp2040064

Jung, W., Shin, H., \& Choi, B. K. (2004, 1). Self-intersection Removal in Triangular Mesh Offsetting. Computer-Aided Design and Applications, 1, 477-484. doi:10.1080/16864360.2004.10738290

Kao, J.-h. (1998). Optimal Motion Planning For Deposition In Layered Manufacturing. Proceedings of DETC'98 1998 ASME Design Engineering Technical Conferences September, (pp. 13-16).

Kapil, S., Negi, S., Joshi, P. V., Sonwane, J., Sharma, A., Bhagchandani, R. K., \& Karunakaran, K. P. (2017). 5-Axis Slicing Methods for Additive Manufacturing Process. Proceedings of the 28th Annual International Solid Freeform Fabrication Symposium - An Additive Manufacturing Conference, (pp. 1886-1896). Retrieved from https://pdfs.semanticscholar.org/1df4/8fb9e0bf71932c829a3d5c232a33ba2db1f7. pdf

Kim, S.-J., Lee, D.-Y., \& Yang, M.-Y. $(2004,1)$. Offset Triangular Mesh Using the Multiple Normal Vectors of a Vertex. Computer-Aided Design and Applications, 1, 285-291. doi:10.1080/16864360.2004.10738269

King, W. E., Anderson, A. T., Ferencz, R. M., Hodge, N. E., Kamath, C., Khairallah, S. A., \& Rubenchik, A. M. $(2015,12)$. Laser powder bed fusion additive manufacturing of metals physics, computational, and materials challenges. Applied Physics Reviews, 2, 041304. doi:10.1063/1.4937809 
Kingsley, J. (2018, 10 10). Adding it up: The economic impact of additive manufacturing. Retrieved from https://eiuperspectives.economist.com/technology-innovation/adding-it-economic-impact-additive-manufacturing

Ko, K. H. (2016). Origins of human intelligence: The chain of tool-making and brain evolution. ANTHROPOLOGICAL NOTEBOOKS, 22, 5-22.

Kobbelt, L. P., Botsch, M., Schwanecke, U., \& Seidel, H.-P. (2001). Feature sensitive surface extraction from volume data. Proceedings of the 28th annual conference on Computer graphics and interactive techniques - SIGGRAPH 01. ACM Press. doi:10.1145/383259.383265

Kollmannsberger, S., Özcan, A., Carraturo, M., Egger, J., Schröder, A., \& Rank, E. (2017). A multi-level model for the simulation of AM processes. Munich, Germany. Retrieved from http://www.eccomas.org/ cvdata/cntr1/spc10/dtos/img/mdia/simam17_bookofabstracts.pdf

Kuhn, J. B. (2015). The innovation station : a 3D printing vending machine for UT Austin students. Master's thesis, The University of Texas at Austin. Retrieved from http://hdl.handle.net/2152/31972

Lee, E. $(2003,5)$. Contour offset approach to spiral toolpath generation with constant scallop height. Computer-Aided Design, 35, 511-518. doi:10.1016/s0010-4485(01)00185-3

Lee, K., \& Jee, H. (2015, 12). Slicing algorithms for multi-axis 3-D metal printing of overhangs. Journal of Mechanical Science and Technology, 29, 5139-5144. Retrieved from https://doi.org/10.1007/s12206015-1113-y

Lensgraf, S., \& Mettu, R. R. (2016, 5). Beyond layers: A 3D-aware toolpath algorithm for fused filament fabrication. 2016 IEEE International Conference on Robotics and Automation (ICRA). IEEE. doi:10.1109/ icra.2016.7487546

Lewis, J. A. (2006, 11). Direct Ink Writing of 3D Functional Materials. Advanced Functional Materials, 16, 2193-2204. doi:10.1002/adfm.200600434

Li, C., Liu, Z. Y., Fang, X. Y., \& Guo, Y. B. (2018). Residual Stress in Metal Additive Manufacturing. Procedia CIRP, 71, 348-353. doi:10.1016/j.procir.2018.05.039

Liou, F., Ruan, J., \& E Sparks, T. (2010, 1). Multi-Axis Planning System (MAPS) for Hybrid Laser Metal Deposition Processes. Proceedings of the 21st Annual International Solid Freeform Fabrication Symposium. Retrieved from https://pdfs.semanticscholar.org/bb7f/80c0dd02679833ee2fbc3f476cdd54257a15. pdf

Locker, A. (2019). 2019 Best 3D Slicer Software for 3D Printers (Most are Free). Retrieved 10 30, 2019, from https://all3dp.com/1/best-3d-slicer-software-3d-printer/\#cura

Lorensen, W. E., \& Cline, H. E. (1987, 8). Marching cubes: A high resolution 3D surface construction algorithm. ACM SIGGRAPH Computer Graphics, 21, 163-169. doi:10.1145/37402.37422

Lowry, S. (2018). Solutions to 3D printing temperature overheating. Retrieved August 9, 2019, from https:// www.gearbest.com/blog/how-to/solutions-to-3d-printing-temperature-overheating-2913

Luo, Z., \& Zhao, Y. (2018, 5). A survey of finite element analysis of temperature and thermal stress fields in powder bed fusion Additive Manufacturing. Additive Manufacturing, 21, 318-332. doi:10.1016/j. addma.2018.03.022

Ma, L., \& Bin, H. (2006, 8). Temperature and stress analysis and simulation in fractal scanning-based laser sintering. The International Journal of Advanced Manufacturing Technology, 34, 898-903. doi:10.1007/ s00170-006-0665-5 
ManufacturingGuide. (n.d.). Laminated Object Manufacturing, LOM. Retrieved August 10, 2019, from https:// www.manufacturingguide.com/en/laminated-object-manufacturing-lom

Masood, S. H., \& Song, W. Q. (2004, 10). Development of new metal/polymer materials for rapid tooling using Fused deposition modelling. Materials \& Design, 25, 587-594. doi:10.1016/j.matdes.2004.02.009

Matos, F., \& Jacinto, C. $(2019,1)$. Additive manufacturing technology: mapping social impacts. Journal of Manufacturing Technology Management, 30, 70-97. doi:10.1108/jmtm-12-2017-0263

Matos, F., Godina, R., Jacinto, C., Carvalho, H., Ribeiro, I., \& Peças, P. (2019, 7). Additive Manufacturing: Exploring the Social Changes and Impacts. Sustainability, 11, 3757. doi:10.3390/su11143757

McCue, I., Peitsch, C., Montalbano, T., Lennon, A., Sopcisak, J., Trexler, M. M., \& Storck, S. (2019, 9). Scalable laser powder bed fusion processing of nitinol shape memory alloy. MRS Communications, 9, 1214-1220. doi:10.1557/mrc.2019.134

McDonald, D. (2013). The Firm: The Story of McKinsey and Its Secret Influence on American Business. Simon \& Schuster. Retrieved from https://www.amazon.com/Firm-McKinsey-Influence-American-Business/ dp/1439190976?SubscriptionId=AKIAIOBINVZYXZQZ2U3A\&tag=chimbori05-20\&linkCode=xm2\&camp $=2025 \&$ creative $=165953 \&$ creativeASIN $=1439190976$

McFarland, M. (2018, 10 30). Segway was supposed to change the world. Two decades later, it just might. Retrieved from https://edition.cnn.com/2018/10/30/tech/segway-history/index.html

McMillan, M., Leary, M., \& Brandt, M. (2017, 10). Computationally efficient finite difference method for metal additive manufacturing: A reduced-order DFAM tool applied to SLM. Materials \& Design, 132, 226-243. doi:10.1016/j.matdes.2017.06.058

Megahed, M., Mindt, H.-W., N’Dri, N., Duan, H., \& Desmaison, O. (2016, 2). Metal additive-manufacturing process and residual stress modeling. Integrating Materials and Manufacturing Innovation, 5, 61-93. doi:10.1186/s40192-016-0047-2

Michaleris, P. (2014, 9). Modeling metal deposition in heat transfer analyses of additive manufacturing processes. Finite Elements in Analysis and Design, 86, 51-60. doi:10.1016/j.finel.2014.04.003

Milewski, J. O., Lewis, G. K., Thoma, D. J., Keel, G. I., Nemec, R. B., \& Reinert, R. A. (1998, 3). Directed light fabrication of a solid metal hemisphere using 5-axis powder deposition. Journal of Materials Processing Technology, 75, 165-172. doi:10.1016/s0924-0136(97)00321-x

Najmon, J. C., Raeisi, S., \& Tovar, A. (2019). Review of additive manufacturing technologies and applications in the aerospace industry. In Additive Manufacturing for the Aerospace Industry (pp. 7-31). Elsevier. doi:10.1016/b978-0-12-814062-8.00002-9

Ngo, T. D., Kashani, A., Imbalzano, G., Nguyen, K. T., \& Hui, D. (2018, 6). Additive manufacturing (3D printing): A review of materials, methods, applications and challenges. Composites Part B: Engineering, 143, 172-196. doi:10.1016/j.compositesb.2018.02.012

Pan, Y., Zhou, C., Chen, Y., \& Partanen, J. (2014, 3). Multitool and Multi-Axis Computer Numerically Controlled Accumulation for Fabricating Conformal Features on Curved Surfaces. Journal of Manufacturing Science and Engineering, 136, 031007--031007-14. doi:10.1115/1.4026898

Panchagnula, J. S., \& Simhambhatla, S. $(2016,4)$. Inclined slicing and weld-deposition for additive manufacturing of metallic objects with large overhangs using higher order kinematics. Virtual and Physical Prototyping, 11, 99-108. doi:10.1080/17452759.2016.1163766 
Papacharalampopoulos, A., Bikas, H., \& Stavropoulos, P. (2018). Path planning for the infill of 3D printed parts utilizing Hilbert curves. Procedia Manufacturing, 21, 757-764. doi:10.1016/j.promfg.2018.02.181

Parteli, E. J., \& P_schel, T. $(2016,1)$. Particle-based simulation of powder application in additive manufacturing. Powder Technology, 288, 96-102. doi:10.1016/j.powtec.2015.10.035

Parvizian, J., D_ster, A., \& Rank, E. (2007, 4). Finite cell method. Computational Mechanics, 41, 121-133. doi:10.1007/s00466-007-0173-y

Paul, R., Anand, S., \& Gerner, F. (2014, 3). Effect of Thermal Deformation on Part Errors in Metal Powder Based Additive Manufacturing Processes. Journal of Manufacturing Science and Engineering, 136, 031009. doi:10.1115/1.4026524

Peters, B., \& Pozzetti, G. (2017). Flow characteristics of metallic powder grains for additive manufacturing. (F. Radjai, S. Nezamabadi, S. Luding, \& J. Y. Delenne, Eds.) EPJ Web of Conferences, 140, 13001. doi:10.1051/epjconf/201714013001

Piegl, L. A., \& Tiller, W. (1999, 2). Computing offsets of NURBS curves and surfaces. Computer-Aided Design, 31, 147-156. doi:10.1016/s0010-4485(98)00066-9

Pirch, N., Niessen, M., Linnenbrink, S., Schopphoven, T., Gasser, A., Poprawe, R., . . Schulz, W. (2018, 8). Temperature field and residual stress distribution for laser metal deposition. Journal of Laser Applications, 30, 032503. doi:10.2351/1.5040634

Ponche, R., Kerbrat, O., Mognol, P., \& Hascoet, J.-Y. (2014, 8). A novel methodology of design for Additive Manufacturing applied to Additive Laser Manufacturing process. Robotics and Computer-Integrated Manufacturing, 30, 389-398. doi:10.1016/j.rcim.2013.12.001

Rajan, V. T., Srinivasan, V., \& Tarabanis, K. A. (2001, 12). The optimal zigzag direction for filling a two-dimensional region. Rapid Prototyping Journal, 7, 231-241. doi:10.1108/13552540110410431

Rane, K., \& Strano, M. (2019, 5). A comprehensive review of extrusion-based additive manufacturing processes for rapid production of metallic and ceramic parts. Advances in Manufacturing, 7, 155-173. doi:10.1007/s40436-019-00253-6

Rank, E., Auricchio, F., Steinmann, P., \& Kollmannsberger, S. (2017). Book of Abstracts. 1st ECCOMAS Thematic Conference on Simulation for Additive Manufacturing., (pp. 592-605). Retrieved from http:// www.eccomas.org/cvdata/cntr1/spc10/dtos/img/mdia/simam17_bookofabstracts.pdf

Recrosio, E. (2017). Q\&A on Stainless Steel Metal Binder Jetting. Retrieved 06 03, 2020, from https://www. sculpteo.com/blog/2017/06/05/qa-on-stainless-steel-metal-binder-jetting/

Ren, K., Chew, Y., Fuh, J. Y., Zhang, Y. F., \& Bi, G. J. (2019, 1). Thermo-mechanical analyses for optimized path planning in laser aided additive manufacturing processes. Materials \& Design, 162, 80-93. doi:10.1016/j.matdes.2018.11.014

Rossignac, J. R., \& Requicha, A. A. (1986, 8). Offsetting operations in solid modelling. Computer Aided Geometric Design, 3, 129-148. doi:10.1016/0167-8396(86)90017-8

Roth, G. A., Geraci, C. L., Stefaniak, A., Murashov, V., \& Howard, J. (2019, 3). Potential occupational hazards of additive manufacturing. Journal of Occupational and Environmental Hygiene, 16, 321-328. doi:1 $0.1080 / 15459624.2019 .1591627$

Ruan, J., Sparks, T. E., Panackal, A., Liou, F. W., Eiamsa-ard, K., Slattery, K., . . Kinsella, M. (2006, 9). Automated Slicing for a Multiaxis Metal Deposition System. Journal of Manufacturing Science and Engineering, 129, 303-310. doi:10.1115/1.2673492 
Sciancalepore, C., Bondioli, F., Gatto, A., Defanti, S., Denti, L., \& Bassoli, E. (2017, 9). DREAM: Driving up reliability and efficiency of additive manufacturing. 2017 IEEE 3rd International Forum on Research and Technologies for Society and Industry (RTSI). IEEE. doi:10.1109/rtsi.2017.8065979

Sethian, J. A. (1999, 1). Fast Marching Methods. SIAM Review, 41, 199-235. doi:10.1137/s0036144598347059

Siemens Digital Industries Software. (2019). Siemens introduces AM Path Optimizer technology integrated in NX for additive manufacturing. Retrieved 04 19, 2020, from https://www.plm.automation.siemens. com/global/en/our-story/newsroom/alert-additive-manufacturing-path-optimizer/66937

Simplify3D. (n.d.). Overheating. Retrieved August 9, 2019, from https://www.simplify3d.com/support/ print-quality-troubleshooting/overheating/

Simplify3D Overheating. (n.d.). Overheating. Retrieved August 9, 2019, from https://www.simplify3d.com/ support/print-quality-troubleshooting/overheating/

Simplify3D. (n.d.). Poor Surface Above Supports. Retrieved August 9, 2019, from https://www.simplify3d. com/support/print-quality-troubleshooting/poor-surface-above-supports/

Simufact. (2019). Simufact Improves Quality with New Dedicated Simulation Solution for Direct Energy Deposition 3D Printing. Retrieved 04 19, 2020, from https://www.mscsoftware.com/node/9619

Singh, A. K., \& Chauhan, S. $(2016,10)$. Technique to Enhance FDM 3D Metal Printing. Bonfring International Journal of Industrial Engineering and Management Science, 6, 128-134. doi:10.9756/bijiems.7574

Singh, P., \& Dutta, D. (2001). Multi-Direction Slicing for Layered Manufacturing. Journal of Computing and Information Science in Engineering, 1, 129-142. doi:10.1115/1.1375816

Singh, P., \& Dutta, D. (2002). Offset Slices for Layered Manufacturing. Volume 1: 22nd Computers and Information in Engineering Conference. ASME. doi:10.1115/detc2002/cie-34493

Singh, P., \& Dutta, D. (2003, 8). Multi-Direction Layered Deposition _ An Overview of Process Planning Methodologies. Proceedings of the 14th Annual International Solid Freeform Fabrication Symposium.

Singh, P., \& Dutta, D. (2008). Offset Slices for Multidirection Layered Deposition. Journal of Manufacturing Science and Engineering, 130, 011011. doi:10.1115/1.2783217

Sjodin, B. (2018). What's The Difference Between FEM, FDM, and FVM? Retrieved 12 26, 2019, from https:// www.machinedesign.com/3d-printing-cad/fea-and-simulation/article/21832072/whats-the-difference-between-fem-fdm-and-fvm

Slic3r. (n.d.). About Slic3r. Retrieved 10 30, 2019, from https://slic3r.org/about/

Smyth, E., \& Vanclay, F. (2017, 1). The Social Framework for Projects: a conceptual but practical model to assist in assessing, planning and managing the social impacts of projects. Impact Assessment and Project Appraisal, 35, 65-80. doi:10.1080/14615517.2016.1271539

Statista Research Department. (2016, 10 13). Global unit shipments of 3D printers from 2015 to $2020^{*}$. Retrieved from https://www.statista.com/statistics/370297/worldwide-shipments-3d-printers/

Stautner, M. (2006). Simulation und Optimierung der mehrachsigen Fräsbearbeitung. Universität Dortmund. Essen: Vulkan Verlag.

Stockman, T., Schneider, J. A., Walker, B., \& Carpenter, J. S. (2019, 1). A 3D Finite Difference Thermal Model Tailored for Additive Manufacturing. JOM, 71, 1117-1126. doi:10.1007/s11837-019-03338-6 
Sun, Y.-J., Yan, C., Wu, S.-W., Gong, H., \& Lee, C.-H. $(2018$, 9). Geometric simulation of 5-axis hybrid additive-subtractive manufacturing based on Tri-dexel model. The International Journal of Advanced Manufacturing Technology, 99, 2597-2610. doi:10.1007/s00170-018-2577-6

Sundaram, R., \& Choi, J. $(2001,8)$. A Slicing Procedure for 5-Axis Layered Manufacturing. Proceedings of the 12th Annual International Solid Freeform Fabrication Symposium. Retrieved from https://sffsymposium.engr.utexas.edu/Manuscripts/2001/2001-46-Sundaram.pdf

Surazhsky, V., Surazhsky, T., Kirsanov, D., Gortler, S. J., \& Hoppe, H. (2005). Fast exact and approximate geodesics on meshes. ACM SIGGRAPH 2005 Papers on - SIGGRAPH 05. ACM Press. doi:10.1145/1186822.1073228

Surmann, T., Ungemach, E., Zabel, A., Joliet, R., \& Schr_der, A. (2011, 4). Simulation of the Temperature Distribution in NC-Milled Workpieces. Advanced Materials Research, 223, 222-230. doi:10.4028/www. scientific.net/amr.223.222

Takahashi, D. (2018, 9 11). Newzoo: Smartphone users will top 3 billion in 2018, hit 3.8 billion by 2021. Retrieved from https://venturebeat.com/2018/09/11/newzoo-smartphone-users-will-top-3-billionin-2018-hit-3-8-billion-by-2021/

Tannehill, J. C., Anderson, D. A., \& Pletcher, R. H. (1997, 4 11). Computational Fluid Mechanics and Heat Transfer, Second Edition. TAYLOR \& FRANCIS. Retrieved from https://www.ebook.de/de/product/4216212/john_c_tannehill_dale_a_anderson_richard_h_pletcher_computational_fluid_mechanics_and_heat_transfer_second_edition.html

The Virtual Foundry. (n.d.). The Virtual Foundry. Retrieved 04 18, 2020, from www.thevirtualfoundry.com

The White House. (2013, 2 12). Remarks by the President in the State of the Union Address. Retrieved from https://obamawhitehouse.archives.gov/the-press-office/2013/02/12/remarks-president-state-union-address

Thompson, A. (2018). Surface texture measurement of metal additively manufactured parts by X-ray computed tomography. PhD Thesis, Centre of Additive Manufacturing. doi:10.13140/RG.2.2.26204.03207

Thompson, M. K., Moroni, G., Vaneker, T., Fadel, G., Campbell, R. I., Gibson, I., .. Martina, F. (2016). Design for Additive Manufacturing: Trends, opportunities, considerations, and constraints. CIRP Annals, 65, 737-760. doi:10.1016/j.cirp.2016.05.004

Tumbleston, J. R., Shirvanyants, D., Ermoshkin, N., Janusziewicz, R., Johnson, A. R., Kelly, D., . . DeSimone, J. M. (2015, 3). Continuous liquid interface production of 3D objects. Science, 347, 1349-1352. doi:10.1126/science.aaa2397

Udroiu, R., Braga, I., \& Nedelcu, A. (2019, 3). Evaluating the Quality Surface Performance of Additive Manufacturing Systems: Methodology and a Material Jetting Case Study. Materials, 12, 995. doi:10.3390/ ma12060995

UPS. (2016). 3D Printing: The Next Revolution in Industrial Manufacturing. Retrieved from https://www. ups.com/media/en/3D_Printing_executive_summary.pdf

Valente, M., Sibai, A., \& Sambucci, M. (2019, 9). Extrusion-Based Additive Manufacturing of Concrete Products: Revolutionizing and Remodeling the Construction Industry. Journal of Composites Science, 3, 88. doi: $10.3390 /$ jcs 3030088

Vastola, G., Zhang, G., Pei, Q. X., \& Zhang, Y.-W. (2016, 10). Controlling of residual stress in additive manufacturing of Ti6Al4V by finite element modeling. Additive Manufacturing, 12, 231-239. doi:10.1016/j. addma.2016.05.010 
Wang, W. M., Zanni, C., \& Kobbelt, L. $(2016,5)$. Improved Surface Quality in 3D Printing by Optimizing the Printing Direction. Computer Graphics Forum, 35, 59-70. doi:10.1111/cgf.12811

Wang, X., Zhang, H., Wang, G., \& Wu, L. (2014, 8). Adaptive Slicing for Multi-Axis Hybrid Plasma Deposition and Milling. Proceedings of the 25th Annual International Solid Freeform Fabrication Symposium. The 25th Solid Freeform Fabrication (SFF) Symposium. Retrieved from https://sffsymposium.engr. utexas.edu/sites/default/files/2014-100-Wang.pdf

Wasser, T., Jayal, A. D., \& Pistor, C. (1999). Implementation and Evaluation of Novel Buildstyles in Fused Deposition Modeling (FDM). The University of Texas at Austin. doi:10.26153/TSW/722

Watson, A. A. (1994). Country Furniture. Globe Pequot Press. Retrieved from https://books.google.de/ books?id=VxBFPgAACAAJ

Wikipedia. (n.d.). FTCS scheme. Retrieved 12 26, 2019, from https://en.wikipedia.org/wiki/FTCS_scheme

Wikipedia. (n.d.). Liberator (gun). Retrieved August 2, 2019, from https://en.wikipedia.org/wiki/Liberator_(gun)

Wits, W. W., Carmignato, S., Zanini, F., \& Vaneker, T. H. (2016). Porosity testing methods for the quality assessment of selective laser melted parts. CIRP Annals, 65, 201-204. doi:10.1016/j.cirp.2016.04.054

Wulle, F., Coupek, D., Sch_ffner, F., Verl, A., Oberhofer, F., \& Maier, T. (2017, 1). Workpiece and Machine Design in Additive Manufacturing for Multi-Axis Fused Deposition Modeling. Procedia CIRP, 60, 229234. Retrieved from http://www.sciencedirect.com/science/article/pii/S2212827117300471

Xiduo, X., Jijun, L., \& Hong, Z. (2009, 11). A new NURBS offset curves and surfaces algorithm based on different geometry shape. 2009 IEEE 10th International Conference on Computer-Aided Industrial Design \& Conceptual Design. IEEE. doi:10.1109/caidcd.2009.5374927

Yang, D. O., \& Feng, H.-Y. $(2008,1)$. Machining Triangular Mesh Surfaces via Mesh Offset Based Tool Paths. Computer-Aided Design and Applications, 5, 254-265. doi:10.3722/cadaps.2008.254-265

Yang, Y., Loh, H. T., Fuh, J. Y., \& Wang, Y. G. (2002, 3). Equidistant path generation for improving scanning efficiency in layered manufacturing. Rapid Prototyping Journal, 8, 30-37. doi:10.1108/13552540210413284

Yap, C. Y., Chua, C. K., Dong, Z. L., Liu, Z. H., Zhang, D. Q., Loh, L. E., \& Sing, S. L. (2015, 12). Review of selective laser melting: Materials and applications. Applied Physics Reviews, 2, 041101. doi:10.1063/1.4935926

Yasa, E., Poyraz, O., Solakoglu, E. U., Akbulut, G., \& Oren, S. (2016). A Study on the Stair Stepping Effect in Direct Metal Laser Sintering of a Nickel-based Superalloy. Procedia CIRP, 45, 175-178. doi:10.1016/j. procir.2016.02.068

Yin, S., Cavaliere, P., Aldwell, B., Jenkins, R., Liao, H., Li, W., \& Lupoi, R. (2018, 5). Cold spray additive manufacturing and repair: Fundamentals and applications. Additive Manufacturing, 21, 628-650. doi:10.1016/j.addma.2018.04.017

Zhang, J., \& Liou, F. (2004). Adaptive Slicing for a Multi-Axis Laser Aided Manufacturing Process. Journal of Mechanical Design, 126, 254-261. doi:10.1115/1.1649966 
